\begin{abstract}
Title of Dissertation: $\quad$ DETECTING THE EBL ATTENUATION OF BLAZARS WITH GLAST

Luis C. Reyes, Doctor of Philosophy, 2007

Dissertation directed by: Adj. Professor Steven M. Ritz

Department of Physics
\end{abstract}

The Large Area Telescope (LAT) on board GLAST (Gamma-ray Large Area Space Telescope) due for launch in late 2007 will study the gamma-ray sky in the energy range $20 \mathrm{MeV}$ to $>300 \mathrm{GeV}$. GLAST-LAT's improved sensitivity with respect to previous missions will increase the number of known gamma-ray blazars from about 100 to thousands, with redshifts up to $\mathrm{z} \sim 3-5$. Since $\gamma$-rays with energy above 10 $\mathrm{GeV}$ interact via pair-production with photons from the Extragalactic Background Light (EBL), the systematic attenuation of GLAST-detected blazars as a function of redshift would constitute and effective and unique probe of the optical-UV EBL density and its evolution over cosmic history.

Analysis techniques introduced in this dissertation make use of the large number of blazars detected by GLAST to study the collective behavior of their spectra as a 
function of redshift. These techniques are shown to offer powerful ways to help separate the common level of attenuation due to the EBL from the intrinsic peculiarities of individual blazars.

The capability of GLAST to perform these measurements depends in great measure on the acceptance of the instrument to high energy $\gamma$-rays $(E>10 \mathrm{GeV})$, which in previous space-experiments has been drastically reduced due to backsplash self-veto. This dissertation includes a study of the backsplash effect as measured with flightlike detectors during a beam test of the LAT calibration unit. This analysis was used to verify the capabilities of the GLAST simulations tools to reproduce backsplash effects. 


\section{DETECTING THE EBL ATTENUATION OF BLAZARS WITH GLAST}

by

Luis C. Reyes

Dissertation submitted to the Faculty of the Graduate School of the University of Maryland, College Park in partial fulfillment of the requirements for the degree of

Doctor of Philosophy

2007

Advisory Committee:

Professor Gregory W. Sullivan, Chair

Adj. Professor Steven M. Ritz, Co-Chair/Advisor

Professor Jordan A. Goodman

Professor Kara Hoffman

Professor M. Colleman Miller 
To my grandparents, Carlos and Margot 


\section{Acknowledgements}

This dissertation has been possible thanks to hundreds of people working on GLAST, from which I have benefited immensely. Thus, I would like to thank the scientists, engineers, programmers and all people involved in the GLAST mission. I also want to thank the funding agencies and member institutions for their support.

My heartfelt thanks to my advisor, Steve Ritz. I am very grateful for his dedication and wholeheartedness towards my formation as a scientist. I have been most fortunate to have Steve as my advisor. In addition to all the constant advice and support, I want to thank Steve for encouraging me to reach out and participate in many different efforts of the LAT collaboration. I also want to thank him for reading so carefully this dissertation; his comments have made it much better.

Julie McEnery has helped me enormously during my journey as a graduate student. I am very grateful for her support and encouragement. Julie was always earnest to answer my questions and to provide advice in general. I also want to thank her for helping me to prepare talks and for carefully reading this dissertation.

I would like to thank Bill Atwood, Jim Chiang, Leon Rochester, Tracy Usher, Toby Burnett, Heather Kelly, Eric Charles, and Seth Digel, among others, for developing analysis and simulation tools that have been used in this dissertation. I am also grateful to the groups led by Paolo Giommi and Gino Tosti for the AGN simulation products they have provided, and which were used in this dissertation.

I want to give due credit to my co-authors of the flux-ratio paper, Andrew Chen 
and Steve Ritz. The three of us acknowledge useful conversations with Bill Atwood, who first suggested using the large statistics of GLAST blazars to look for systematic effects due to EBL attenuation as a function of redshift.

I have enjoyed very much my stay at NASA's Goddard Space Flight Center where I have learned so much. I am very grateful to Alex Moiseev who guided me through many ACD analyses, including backsplash. I also want to thank Dave Thompson, Bob Hartman, Neil Gehrels, Floyd Stecker, Sasha Kashlinsky and Bill Daniels for many useful and interesting discussions. Thanks to Sasha for reading parts of this dissertation and for providing useful comments.

I would like to thank the members of the Blazar and other $A G N$ science group for useful feedback and discussions regarding my analysis of EBL attenuation of blazars. In particular, I want to acknowledge the constant support and encouragement by Benoit Lott.

Thanks to all the people involved in the beam test at CERN of 2006. In particular, I would like to thank Luca Latronico, Philippe Bruel and Benoit Lott for their guidance of the group. I also want to acknowledge the hard work by our European collaborators, especially the Pisa group, during the preparation of the beam test.

During the last few years it was a pleasure to work closely with the SVAC group at SLAC. Thanks to Eduardo do Couto e Silva, Anders Borgland and Eric Charles for their guidance and for thinking of me when some extra help was needed. The trust they deposited on me was a big confidence boost.

I want to thank my officemates David Wren and Navid Golpayegani for the companionship. Navid has been a constant source of technological support during my ongoing learning of the subtleties of the GLAST software. Without him I would still be trying to compile the code. Thanks also to Heather Kelly and Richard Dubois for answering so many questions with such a great disposition.

Thanks a lot to the departments of Physics and Astronomy at University of Mary- 
land for all the academic and administrative support. In particular, I am very grateful to Greg Sullivan, Jane Hessing, Linda O'Hara, Nick Chant, Susan Lehr, and John Trasco. My classmates at College Park provided an environment full of comradeship and fun where it was pleasant to work on homeworks and to study for the quals. Thanks to Nick, Manolis, Patrick, Santiago, Guido, Dan, Beth, Chad and Mike.

Moving away from family and friends to a foreign country became less hard thanks to great friends such as Biviana, Samir, Olga, Juan David, Ana Maria, Juan Gabriel, Ricardo and Maria Antonieta. Thanks for the camaraderie.

I want to thank my girlfriend Maral for her support and encouragement. Thanks for her patience during all these years of student life and for putting up with me during the writing of this dissertation. Her love and companionship have made all the difference.

Thanks to my parents and all my extended family for their love and support. I want to acknowledge the example of hard work and inner drive that I have received from my grandparents and parents. As far as I may go in life would be just the continuation of the journey they started, which I am proud to follow.

Finally, thanks to God for showing the way. 


\section{Table of Contents}

List of Tables $\quad$ xii

List of Figures $\quad$ xiii

1 Introduction $\quad 1$

1.1 Brief Introduction to $\gamma$-ray Astrophysics . . . . . . . . . . . . . . . 1

1.1.1 The Physics of $\gamma$-rays . . . . . . . . . . . . . . . 2

1.1.1.1 Production .................. 2

1.1.1.2 Interactions of $\gamma$-rays in Matter . . . . . . . 6

1.1.1.3 Interaction of $\gamma$-rays with Radiation Fields . . . . . 8

1.1.2 Detection of Astronomical $\gamma$-rays . . . . . . . . . . . . 10

1.1.2.1 Detection of $\gamma$-rays in Space . . . . . . . . . . 11

1.1.2.2 Detection of $\gamma$-rays on the Ground . . . . . . . . 12

1.1.3 The Legacy of CGRO and EGRET . . . . . . . . . . 15

1.2 Gamma-ray Emission of Blazars . . . . . . . . . . . . . . . . . . 17

1.2.1 AGN Properties and Definition ............ 17

1.2.2 AGN Taxonomy . . . . . . . . . . . . . . . 20

1.2.3 The AGN Paradigm . . . . . . . . . . . . . 23

1.2.4 Gamma-ray Emission . . . . . . . . . . . . . . 26

1.2.4.1 Observations ................. 26 
1.2.4.2 Spectral Energy Distributions and the Blazar Sequence 28

1.2.4.3 Gamma-ray Emission in Blazars . . . . . . . . . . . 31

1.3 Dissertation Outline . . . . . . . . . . . . . . . . 35

$\begin{array}{lll}2 & \text { Extragalactic Background Light } & 37\end{array}$

2.1 What is the EBL? . . . . . . . . . . . . . . 37

2.2 EBL Contributors . . . . . . . . . . . . . . . . . . . . . . 39

2.2.1 Conventional contributors . . . . . . . . . . . . . . . 39

2.2.1.1 Emission from Normal Stellar Populations . . . . . . 40

2.2.1.2 Dust emission from galaxies . . . . . . . . . . 43

2.2.2 Additional Contributors . . . . . . . . . . . . . . . 44

2.2.2.1 Quasars / AGN ............... 44

2.2.2.2 Population III stars . . . . . . . . . . . . . . 44

2.2.2.3 Exotic Sources . . . . . . . . . . . . 46

2.3 Direct Measurements . . . . . . . . . . . . . . . . . 47

2.3.1 UV-Optical . . . . . . . . . . . . . . . . . . . . . . . 49

2.3 .2 IR . . . . . . . . . . . . . . . . 52

2.3.2.1 COBE-DIRBE . . . . . . . . . . . 53

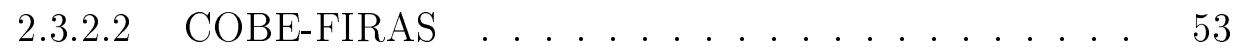

2.3 .2 .3 IRTS-NIRS . . . . . . . . . . . . . . . 54

2.3.2.4 NICMOS .................... 56

2.3.2.5 IRAS, ISO, SCUBA, SPITZER . . . . . . . 56

2.4 EBL Density as a Cosmological and Astrophysical Probe . . . . . . . 57

2.4.1 Total EBL energy . . . . . . . . . . . . . . . 57

2.4.2 Star Formation Rate (SFR) _ . . . . . . . . . . . . 59

2.4.3 Element Production . . . . . . . . . . . . . . . . 61

2.4.4 The connection between Radio and IR backgrounds . . . . . . 62 
2.5 EBL Models . . . . . . . . . . . . . . . . . . . . 63

2.5.1 Description of EBL Models . . . . . . . . . . . . . 63

2.5.1.1 Simple Backward Evolution Models . . . . . . . . . 63

2.5.1.2 Forward Evolution Models . . . . . . . . . . . . . 64

2.5.1.3 Semi-analytical Models . . . . . . . . . . . . 65

2.5.1.4 Chemical Evolution Models . . . . . . . . . . 65

2.5.2 EBL models used for GLAST Simulations . . . . . . . . . . 66

2.5.2.1 Kneiske et al (2004) . . . . . . . . . . 66

2.5.2.2 Primack et al . . . . . . . . . . . . . . 68

2.5.2.3 Stecker et al . . . . . . . . . . . . . 68

2.6 EBL attenuation of gamma-ray sources . . . . . . . . . . . . . . 69

2.6.1 Historical Background . . . . . . . . . . . . . 69

2.6.2 Calculation of the Optical Depth . . . . . . . . . . . 70

2.6.3 Optical Depth calculated from EBL Models . . . . . . . . . 73

2.6.4 Using gamma-ray sources to measure the EBL density . . . . 77

2.6.4.1 Attenuation of $\mathrm{TeV}$ sources by IR-EBL . . . . . . . 77

2.6.4.2 GLAST Prospects ............. 83

3 The Gamma-ray Large Area Space Telescope (GLAST) 85

3.1 The Pair-Conversion Telescope Technique . . . . . . . . . . . . . . 85

3.2 GLAST LAT . . . . . . . . . . . . . . . 87

$3.2 .1 \quad$ LAT ACD . . . . . . . . . . . . . . . 88

3.2.1.1 Design Considerations . . . . . . . . . . . 88

3.2.1.2 ACD Design . . . . . . . . . . . . . 89

3.2.1.3 ACD Electronics . . . . . . . . . . . . . 93

3.2 .2 LAT Tracker . . . . . . . . . . . . . . . . 96

3.2.2.1 Design Considerations . . . . . . . . . . . 96 
3.2.2.2 TKR Design . . . . . . . . . . . . . . . . . 97

3.2.2.3 TKR Electronics . . . . . . . . . . . . . . . . . 102

3.2.3 LAT Calorimeter . . . . . . . . . . . . . . 106

3.2.3.1 Design Considerations . . . . . . . . . . . 106

3.2.3.2 CAL Design . . . . . . . . . . . . . 107

3.2.3.3 CAL Electronics . . . . . . . . . . . . 109

3.2.4 LAT Data Acquisition (DAQ) System . . . . . . . . . . . . . 113

3.2.4.1 Global Trigger . . . . . . . . . . . . . 113

3.2.4.2 Onboard Filter $(\mathrm{OBF}) \ldots \ldots \ldots$

3.3 LAT Simulation . . . . . . . . . . . . . . . . . . . . . 119

3.4 Event Reconstruction . . . . . . . . . . . . . . . . . . . . . 120

3.5 Event Selection and Background Rejection . . . . . . . . . . . 126

3.5.1 Energy Analysis . . . . . . . . . . . . . . . . . 126

3.5.2 PSF Analysis . . . . . . . . . . . . . . . . . . . . 128

3.5.3 Background Rejection . . . . . . . . . . . . . . 129

3.6 Brief overview of Instrument Performance . . . . . . . . . . . . . . . 132

3.6.1 Instrument Response Functions (IRFs) . . . . . . . . . . . . . 132

3.6.1.1 Effective Area . . . . . . . . . . . . 133

3.6.1.2 Point-Spread Function (PSF) . . . . . . . . . 133

3.6.1.3 Energy Resolution . . . . . . . . . . . . 135

3.6.2 Point-Source Sensitivity . . . . . . . . . . . . . 135

3.7 GLAST Burst Monitor $(\mathrm{GBM}) \ldots \ldots \ldots$

4 Beam Test of the LAT Calibration Unit: ACD Backsplash Studies 140

4.1 Beam Test Rationale . . . . . . . . . . . . . . . . . . . . . . . 140

4.1 .1 ACD Backsplash .................. 141

4.2 Beam Test Description . . . . . . . . . . . . . . . . . 143 
4.2.1 LAT Calibration Unit Description . . . . . . . . . . . . . 143

4.2 .2 Beam Description . . . . . . . . . . . . . . . . . . . 145

4.2.3 Experimental Setup . . . . . . . . . . . . . 147

4.2.4 Data Taking Configurations . . . . . . . . . . . . . . 150

4.3 Analysis . . . . . . . . . . . . . . . . . . . 150

4.3.1 Considerations . . . . . . . . . . . . . 150

4.3.2 ACD Calibration . . . . . . . . . . . . . . 152

4.3.3 Measured Backsplash Distribution . . . . . . . . . . . . . . 155

4.3.3.1 Event Selection . . . . . . . . . . . . . 155

4.3.3.2 Results .................. 156

4.3.3.3 Background Considerations . . . . . . . . . . . 161

4.3.4 Monte Carlo Simulations . . . . . . . . . . . . . . . . 163

4.3.5 Comparison of Data and Monte Carlo Simulations . . . . . . . 165

4.3.6 Backsplash Energy Dependence . . . . . . . . . . . . . 166

4.4 Summary and Conclusions . . . . . . . . . . . . . . . . . 171

5 Detecting the EBL Attenuation of Blazars with GLAST $\quad 174$

5.1 The Impact of GLAST on AGN Science . . . . . . . . . . . . . . . . 174

5.1 .1 Blazar Luminosity Function . . . . . . . . . . . . . . 176

5.2 Flux-Ratio Method . . . . . . . . . . . . . . . . . . . . 179

5.2.1 Monte Carlo Simulation . . . . . . . . . . . . . . . . . . 179

5.2.1.1 Simulated Blazar Populations . . . . . . . . . . . 180

$5.2 .1 .2 \quad$ Blazar spectra . . . . . . . . . . . . 185

5.2.1.3 Simulated population of blazars . . . . . . . . 186

5.2 .2 Calculating the Flux ratios . . . . . . . . . . . . . . 189

5.2 .3 Results. . . . . . . . . . . . . . . . . 190

5.3 Spectral Analysis . . . . . . . . . . . . . . . . . . 195 
5.3.1 Monte Carlo Simulation . . . . . . . . . . . . . . . . . . . 197

5.3.1.1 Blazar Population . . . . . . . . . . . 197

5.3.1.2 Blazar Variability . . . . . . . . . . . . 198

5.3.2 Modeling the observed blazar spectrum . . . . . . . . . . . 200

5.3 .3 Spectrum fitting . . . . . . . . . . . . . 205

5.3.3.1 Maximum Likelihood . . . . . . . . . . . 205

5.3.4 Fazio-Stecker Relation . . . . . . . . . . . . . . . 208

5.4 Related Issues . . . . . . . . . . . . . . . . . . . . . . . . . 211

5.4.1 Possible Sources of Bias . . . . . . . . . . . . . 211

5.4.1.1 Selection Effects ............... 211

5.4.1.2 Blazar Variability . . . . . . . . . . . . . 213

5.4.1.3 Spectral Blazar Evolution . . . . . . . . . . . 214

5.4.2 Redshift Determination for GLAST blazars . . . . . . . . . . . 215

5.4.2.1 Existing Blazar Catalogs . . . . . . . . . . . 216

5.4.2.2 Catalogs of Blazar Candidates . . . . . . . . . 218

$5.4 .2 .3 \quad$ Blazar Identification . . . . . . . . . . . . . 219

5.5 The Future . . . . . . . . . . . . . . . . . . . . . . . . . . 221

5.5.1 Unfolding of EBL density from Optical depth . . . . . . . . 221

5.5.2 Joint spectral analysis with ground-based instruments . . . . . 222

5.5.3 Related EBL-Attenuation Phenomena . . . . . . . . . . . 224

5.6 Summary and Conclusions . . . . . . . . . . . . . . . . 227

6 Conclusion $\quad 229$ 


\section{List of Tables}

1.1 Classification of AGN according to their radio-loudness and optical emission lines . . . . . . . . . . . . . . . . . . 25

2.1 Integrated galaxy light measurements . . . . . . . . . . . . . . 50

2.2 Measurements of the optical-UV EBL after foreground subtraction . . 51

2.3 Measurements of the infrared EBL from the study of background fluc-

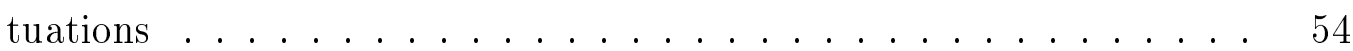

2.4 Compilation of DIRBE measurements of the EBL . . . . . . . . 55

2.5 Integrated galaxy light measurements of the infrared EBL $\quad \ldots . . .58$

2.6 Integrated EBL energy . . . . . . . . . . . . . . . . . 58

4.1 Data configurations during the beam test at CERN SPS in 2006. . . 153

4.2 Electron runs at SPS. . . . . . . . . . . . . . . . . . . 153

4.3 Pedestal mean and width in ADC counts for every ACD tile. . . . . . 159

4.4 Tile parameters obtained from the pulse-height distribution from calibration runs .......................... 160

4.5 Event selection cuts . . . . . . . . . . . . . . . . . . . 164

5.1 Fit parameters and spectral energy cut-offs for blazars at different redshifts . . . . . . . . . . . . . . . . . . . 208 


\section{List of Figures}

1.1 The electromagnetic spectrum . . . . . . . . . . . . . . . . . 1

1.2 Behavior of the pair-production cross section as a function of energy . 9

1.3 Schematic representation of $\gamma$-ray induced showers in the atmosphere and the ground-based instruments that detect them . . . . . . . . 14

1.4 The Compton Gamma-ray Observatory (CGRO) . . . . . . . . . . . . 16

1.5 Optical spectra of a Seyfert Galaxy . . . . . . . . . . . . . . . . 18

1.6 Radio intensity maps for FR1- and FR2-type galaxies . . . . . . . . . 22

1.7 Schematic representation of the radio-loud AGN paradigm . . . . . . 24

1.8 Blazar variability as measured by EGRET . . . . . . . . . . . . . . . 27

1.9 The blazar sequence $\ldots \ldots$. . . . . . . . . . . . . . . . . . . 30

2.1 The EBL spectrum . . . . . . . . . . . . . . . . . . . . . 38

2.2 EBL density evolution as a function of redshift . . . . . . . . . . . 40

2.3 The calculated spectrum of a Pop III star . . . . . . . . . . . . 45

2.4 Compilation of EBL measurements and limits . . . . . . . . . . . . . 48

2.5 Comoving star formation rate density as a function of redshift . . . . 59

2.6 EBL density at various redshifts from the EBL model by Kneiske et al

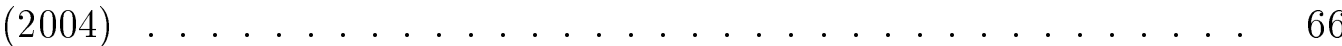

2.7 Optical depth $\tau(E, z)$ according to the EBL models used in the GLAST simulation ....................... . . 74 
2.8 Optical depth as a function of energy at different redshifts . . . . . . 75

2.9 Fazio-Stecker relation for the EBL models used in GLAST simulations. 78

2.10 Compilation of EBL upper limits derived from observations of $\mathrm{TeV}$ blazars ........................... 79

2.11 The spectra of 1ES 1101-232 as observed by the HESS collaboration and after EBL correction . . . . . . . . . . . . . 82

2.12 Upper EBL limit at NIR wavelengths as measured by the HESS collaboration

3.1 The pair-conversion telescope technique . . . . . . . . . . . 86

3.2 The Large Area Telescope $(\mathrm{LAT}) \ldots \ldots$. . . . . . . . . . . . 88

3.3 Picture of an ACD tile . . . . . . . . . . . . . . . . 90

3.4 GLAST ACD tile layout . . . . . . . . . . . . . . . . . . . . 91

3.5 GLAST ACD tile and ribbons layout . . . . . . . . . . . . . . . 91

3.6 Schematic representation of ACD electronics. . . . . . . . . . . . . 94

3.7 Picture of a Silicon-Strip Detector (SSD) . . . . . . . . . . . . . . . . 99

3.8 Picture of a Tracker ladder . . . . . . . . . . . . . . . . . . . . . . 99

3.9 [Schematic representation of a tracker tray] Tracker tray consisting of two silicon panels, converter foil and structural panel (described in detail in [261]). . . . . . . . . . . . . . . . . . . . . . . . . . . 101

3.10 Tracker module . . . . . . . . . . . . . . . . . . . . . . . 101

3.11 GTFE and GTRC electronics chips . . . . . . . . . . . . . . 103

3.12 FAST-OR signal from the tracker GTFEs . . . . . . . . . . . . . . 103

3.13 TKR trigger logic diagram . . . . . . . . . . . . . . . . . . . . . . 104

3.14 GLAST calorimeter crystal $\log \ldots \ldots \ldots$. . . . . . . . . . . 108

3.15 GLAST calorimeter Module . . . . . . . . . . . . . . . 108

3.16 GLAST GCFE functional block diagram . . . . . . . . . . . . . . . 109 
3.17 CAL electronics functional block diagram . . . . . . . . . . . . . . . 111

3.18 CAL trigger signals . . . . . . . . . . . . . . . . . . . . . 112

3.19 Expected average background flux (on-orbit) . . . . . . . . . . . 117

3.20 Schematic representation of LAT event reconstruction . . . . . . . . . 121

3.21 The Kalman filtering process . . . . . . . . . . . . . . . . . . . . . . . 124

3.22 The Kalman smoothing process . . . . . . . . . . . . . . . . . . . . 124

3.23 Residual background rate as a function of energy . . . . . . . . . . . 131

3.24 LAT effective area as a function of photon energy . . . . . . . . . . . 134

3.25 LAT relative effective area as a function of incident angle . . . . . . . 134

3.26 LAT point-spread function $(\mathrm{PSF}) \ldots \ldots \ldots \ldots$

3.27 LAT energy resolution . . . . . . . . . . . . . . . . . . 136

3.28 Reconstructed vs Monte Carlo energies . . . . . . . . . . . . . . 136

3.29 LAT point-source sensitivity . . . . . . . . . . . . . . . . . . 137

3.30 The GLAST Burst Monitor $(\mathrm{GBM}) \ldots \ldots \ldots$

4.1 Schematic representation of ACD backsplash . . . . . . . . . . . . . . 142

4.2 ACD tiles in the LAT calibration unit . . . . . . . . . . . . . . 144

4.3 Dimensions of the ACD tiles in the LAT calibration unit. Courtesy of the beam test group. . . . . . . . . . . . . . . . . . . 146

4.4 Experimental setup at CERN-SPS . . . . . . . . . . . . . . 147

4.5 Calibration Unit orientation with respect to the beam . . . . . . . . . 148

4.6 Calibration unit during different data taking configurations . . . . . . 149

4.7 Pedestal for ACD tile in ADC counts . . . . . . . . . . . . . . . 153

4.8 Pulse-height distribution for an ACD tile exposed to a $150 \mathrm{GeV}$ proton

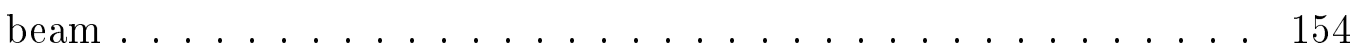

4.9 Calibration unit orientation with respect to the beam. . . . . . . . . 158 
4.10 Measured ACD backsplash signal normalized to the total number of

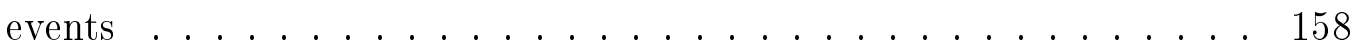

4.11 Measured backsplash distribution for tile 110 . . . . . . . . . . . . 159

4.12 Fraction of backsplash events as a function of beam energy . . . . . . 162

4.13 Event display of a $20 \mathrm{GeV}$ electron incident on the calibration unit . . 164

4.14 Measured backsplash distribution for tile $0 \ldots \ldots$. . . . . . . . 167

4.15 Measured backsplash distribution for tile $110 \ldots \ldots$. . . . . . . . . 168

4.16 Measured backsplash distribution for tile 120 . . . . . . . . . . . . 169

4.17 Measured backsplash distribution for tile 130 . . . . . . . . . . . . 170

4.18 Energy dependence of ACD backsplash . . . . . . . . . . . . . . . . 171

4.19 Estimated LAT ACD backsplash probability as extrapolated from the beam test data . . . . . . . . . . . . . . . . 172

$5.1 \quad$ Blazar $\log (N)-\log (S)$ plot . . . . . . . . . . . . . . 178

5.2 Blazar luminosity function according to Chiang \& Mukherjee (1998) • 182

5.3 Blazar redshift distribution according to Chiang \& Mukherjee (1998) 184

5.4 Number of GLAST detectable blazars as a function of redshift . . . . 188

5.5 Flux ratio scatter plot . . . . . . . . . . . . . . . . . . . 191

5.6 Mean flux ratio as a function of redshift . . . . . . . . . . . . . . . . 192

5.7 Mean observed flux ratio for blazars with a broken power-law spectrum 194

5.8 Mean flux ratio according to the most recent EBL models . . . . . . . 196

5.9 Simulated blazar lightcurve . . . . . . . . . . . . . . . . . . . . 199

5.10 Fit to $e^{-\tau}$ with a function of the form $e^{-E / E_{0}} \ldots \ldots . \ldots . . \ldots 201$

5.11 Fit to $e^{-\tau}$ with a function of the form $e^{-\left(E-E_{b}\right) / P_{1}} \ldots \ldots 203$

5.12 Spectrum and model fit for a simulated $\gamma$-ray blazar at redshift $z=3.23207$

5.13 Spectra and model fits for blazars simulated at different redshifts . . 209

5.14 Scatter plot of the Fazio-Stecker relation for the simulated data . . . 212 
5.15 Redshift distribution for blazars in the 3rd EGRET catalog . . . . . . 217

5.16 Redshift distribution of blazars in the ASDC Catalog of blazars Vol. I 217

5.17 Redshift distribution of FSRQs in the Candidate Gamma-ray Blazar

Survey in the northern sky . . . . . . . . . . . . . . . 220

5.18 Blazar candidates in the ROXA survey . . . . . . . . . . . . 220

5.19 Extended $\mathrm{GeV}-\mathrm{TeV}$ spectrum for a $\gamma$-ray source at $z=0.2 \ldots . . .223$ 


\section{Chapter 1}

\section{Introduction}

\subsection{Brief Introduction to $\gamma$-ray Astrophysics}

Astronomical observations have been an essential source of human knowledge through history, but just recently has the universe been observed at wavelengths outside the visible region of the electromagnetic spectrum. The scientific return for such curiosity has been spectacular. Again and again, observations in a previously unexplored spectral range have opened the window to new objects and new physical phenomena that would have remained hidden otherwise.

Gamma-rays, in particular, belong to the most energetic part of the electromagnetic spectrum, with energies starting at a few hundred keV (fig. 1.1). Gamma-ray telescopes have observed $\gamma$-rays with energies up to tens of $\mathrm{TeV}$, which means that

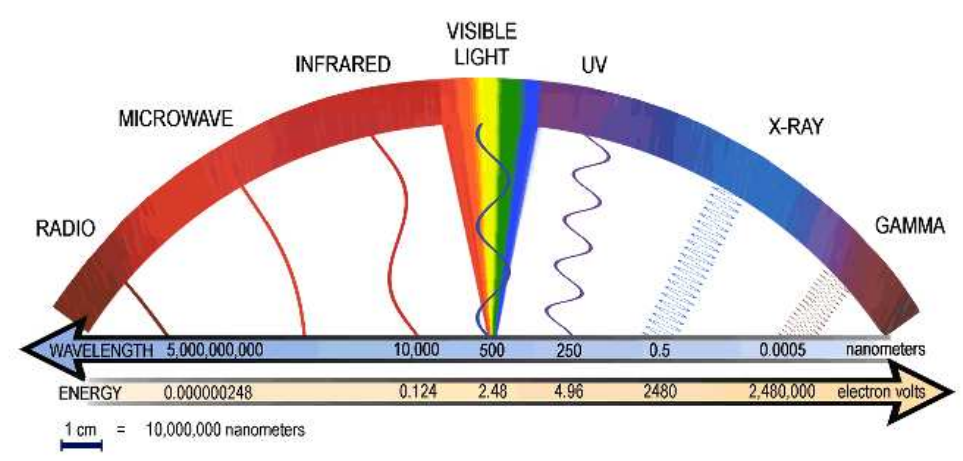

Figure 1.1: The electromagnetic spectrum. Adapted from [202]. 
gamma-ray astronomy covers an energy band more extensive, by far, than the narrow visible band in which astronomy was born.

The large amount of energy carried by every $\gamma$-ray implies that only the most powerful processes in the universe are able to produce significant amounts of gammaray radiation. It is not surprising then, that great efforts are made at the theoretical and experimental level to gain understanding of known astronomical $\gamma$-ray sources such as: the Sun [44], gamma-ray pulsars [207], supernova remnants and pulsar wind nebulae [4, 3], diffuse emission from the galaxy [260], active galactic nuclei (discussed below), gamma-ray bursts[282], and diffuse emission from outside the galaxy [259].

Building upon the legacy of pioneer space and ground based experiments like CGRO, WHIPPLE, HEGRA, and others[157], existing and future instruments continue to revamp our understanding of the $\gamma$-ray universe.

\subsubsection{The Physics of $\gamma$-rays}

Gamma rays (as any other form of electromagnetic radiation) are described by the quantum theory of light, QED (Quantum Electrodynamics [81]). Light can be described as particles propagating with energy $E=h \nu$, or as waves with wavelength $\lambda=c / \nu$. The production and interaction mechanisms of $\gamma$-rays are determined by their very high energy and very short wavelength.

\subsubsection{Production}

Before getting into detail about the different physical processes that produce $\gamma$-rays, it is important to make a distinction between "thermal" and "non-thermal" electromagnetic radiation. Thermal radiation is black-body-type radiation, where the energy density per frequency unit goes to zero for small and large frequencies and its maximum is proportional to the characteristic temperature of the black body (Wien's law). A black body able to produce significant amounts of $\gamma$-ray radiation around 
$10 \mathrm{MeV}$ would require a temperature above $\mathcal{O}\left(10^{10}\right) \mathrm{K}$, which is at least 6 orders of magnitude larger than the typical temperature of a regular star. Objects with such high temperatures are not the norm in the universe, and consequently, most $\gamma$-rays are produced in nonthermal processes, that usually involve charged particles in electromagnetic fields. The fundamental production mechanisms of $\gamma$-rays are outlined below.

\section{Bremsstrahlung and Synchrotron Radiation}

It is a well known fact from electrodynamics that acceleration of charged particles by electric or magnetic fields produces electromagnetic radiation [122]. Key quantities like the total radiation emitted per unit time, the angular distribution and the frequency spectrum, depend on parameters such as the strength and orientation of the external field, and the charge, mass and momentum of the accelerated particle.

Bremsstrahlung - or "braking radiation" - is due to the acceleration of a charged particle in an external electric field. For example, a free electron traversing matter may pass very close to an atomic nucleus, resulting in a sudden acceleration and the loss of electron energy in the form of radiation. The intensity per unit frequency of bremsstrahlung radiation as a function of photon energy $E$ is flat to first approximation in $\log (E)$ up to

$$
E=(\gamma-1) m_{e} c^{2}
$$

where $\gamma$ is the particle Lorentz factor. Emission of photons above this energy is not allowed since the electron has given up all its kinetic energy. Therefore, production of $\gamma$-rays by this mechanism is only possible when dealing with highly relativistic particles.

Synchrotron radiation is due to the change of direction experienced by a charged particle in an external magnetic field. This radiation is emitted in a narrow cone in 
the forward direction, tangent to the trajectory of the particle. Synchrotron radiation extends over a wide range of frequencies and peaks at

$$
\nu=\frac{3}{2} \gamma^{2} \times \frac{e B}{2 \pi m \sin \theta}
$$

where $\gamma$ is the Lorentz factor, $e$ is the particle electric charge, $m$ is the particle mass, $B$ is the magnetic field and $\theta$ is the angle between the particle trajectory and the direction of the magnetic field. For example, synchrotron radiation peaks at radio energies for cosmic rays $(\sim 1 \mathrm{GeV})$ in the interstellar magnetic field $\left(\sim 10^{-6} \mathrm{G}\right)$, while high energy electrons $(10 \mathrm{GeV})$ being accelerated in high energy colliders $\left(\sim 10^{3} \mathrm{G}\right)$ produce most of their emission in the optical band. Although synchrotron radiation is not the dominant form of direct production of $\gamma$-rays in the universe, radio to X-ray photons produced by synchrotron emission in AGN jets are an essential ingredient to the production of high energy photons by inverse Compton scattering as will be discussed in section 1.2.4.3.

\section{Inverse Compton Scattering}

Inverse Compton scattering occurs when low energy photons are scattered up in energy by relativistic charged particles. Given the right circumstances, the photon could acquire a large amount of energy from the collision and become a $\gamma$-ray. In the case of very energetic electrons, the energy of the up-scattered photon can be approximated to

$$
E_{\gamma} \simeq \frac{4}{3} \gamma^{2} E_{p h}
$$


where $\gamma$ is the Lorentz factor or the electron and $E_{p h}$ is the energy of the original photon [59]. Therefore, astrophysical sources with high photon densities ${ }^{1}$ and high energy particles are prime sources of $\gamma$-rays.

\section{Nuclear Transitions}

The allowed energy states of atomic nuclei are discrete and usually lie a few $\mathrm{MeV}$ apart. Thus, $\gamma$-rays with energy equal to any of these gaps can be absorbed or emitted during nuclear transitions ${ }^{2}$. One of the signatures of this process is that the gammaray spectrum is composed of discrete lines instead of a continuum. Significant $\gamma$-ray emission by this mechanism requires a large number of atomic nuclei to be disturbed into excited states.

\section{Pion Decay}

Hadronic interactions such as cosmic ray protons colliding with gas nuclei produce copious amounts of pions. Neutral pions $\left(\pi^{0}\right)$ are unstable particles and decay into a pair of $\gamma$-ray photons with energy $\sim 70 \mathrm{MeV}$, half the rest mass of the $\pi^{0}$. Because of the momentum distribution of the original pions, pion decay is observed as a $\gamma$-ray bump, that in the case of beamed emission towards the observer is Doppler-shifted to higher energies [59].

In a related process, $\gamma$-rays are produced by high energy protons via photopion production. In this process protons interact with ambient photons to produce $e^{-}-e^{+}$ pairs and pions, that in turn produce $\gamma$-rays via inverse Compton scattering and pion decay $\left(\pi^{0} \rightarrow \gamma+\gamma\right)$ if an ambient radiation field is present.

\footnotetext{
${ }^{1} \mathrm{~A}$ caveat is required. For very high photon densities the optical depth $\tau$ due to $\gamma+\gamma \rightarrow e^{-}+e^{+}$ interactions may become large $(\tau>1)$. In such cases, most $\gamma$-rays will be absorbed. The physics of pair production will be discussed in sec. 1.1.1.2

${ }^{2}$ Although this $\gamma$-ray emission mechanism is not relevant for this dissertation, it is mentioned here for completeness.
} 


\section{Annihilation}

Particle and antiparticle pairs annihilate when encountering each other, and their mass is converted into energy. This energy usually gives origin to two photons (or more, depending on the angular momentum of the particle-antiparticle system). Since the lightest particle is the electron with a rest mass energy of $511 \mathrm{keV}$, it is guaranteed that annihilation processes will radiate photons in the gamma-ray energy regime. Of extreme importance for the field of gamma-ray astrophysics is the possibility of detecting the radiation resulting from the annihilation of hypothesized massive particles [274], which (if they exist) could account for the dark matter in the universe.

\subsubsection{Interactions of $\gamma$-rays in Matter}

Gamma-rays are able to resolve matter in their constituent atomic nuclei and electron clouds. Therefore, the passage of an individual photon through matter is a random process, since it depends on the probability that it will encounter an electron or a nucleus in its path. The interaction of $\gamma$-rays in matter is determined by the photon energy and the characteristics of the material (density and atomic number).

\section{Photoelectric Absorption}

In this process, the incident photon is absorbed by an electron in the material, giving it sufficient energy to break loose from the atom. This is the dominant interaction in the energy range $100 \mathrm{eV}-100 \mathrm{keV}$.

\section{Compton Scattering}

When the energy of the photon is between $100 \mathrm{keV}$ and a few $\mathrm{MeV}$, the atomic binding energy of the electrons is small by comparison. After giving part of its energy to an electron, the photon scatters in a different direction. The electron is removed from 
the atom as in the photoelectric effect, but this time it gains a significant amount of kinetic energy.

\section{Pair Production}

Photons passing close to an atomic nucleus will experience the electric field of the protons, and the stronger this field (with higher atomic number $Z$ ), the more likely it is to produce an electron-positron pair. Obviously, this reaction cannot occur if the photon-nucleus system does not have enough energy in the center-of-momentum frame to produce the rest masses of the pair $(\sim 1 \mathrm{MeV})$. Above this energy threshold, pair creation is the dominant interaction of $\gamma$-rays in matter. By momentum conservation, the electron and positron pair are emitted in the forward direction of the incident photon in the nucleus frame, separated by a narrow opening angle that decreases with energy.

\section{Electromagnetic Cascades}

Although not a type of interaction by itself, electromagnetic cascades are a very important phenomenon in the context of $\gamma$-rays passing through matter. When a high energy photon or electron is incident on a thick absorber, it initiates an electromagnetic cascade as successive pair production and bremsstrahlung interactions generate more electrons and photons with lower energy. During the development of the shower, particles undergo Coulomb scattering in the material which causes the shower to spread out in the transverse direction. If the absorber is deep enough, the average particle energy will eventually fall below the critical energy $\left(E_{C}\right)$, and electrons and photons will dissipate their energy by ionization and excitation rather than by the generation of more shower particles. At this point the shower has reached its maximum and begins to decrease [217]. The critical energy depends on the absorber 
material (through the atomic number $Z$ ) and can be approximated by [74]:

$$
E_{C}(\mathrm{MeV})=\frac{610}{(Z+1.24)}
$$

Furthermore, the amount of matter traversed by a high-energy photon is conveniently expressed in units of radiation length $\left(X_{0}\right)$ [74], with one radiation length being equal to $7 / 9$ of the mean free path for pair production by a high-energy photon $^{3}$. One of the important observables of an electromagnetic shower is the shower maximum $t_{\max }$, which is measured in units of radiation length and occurs at [74]:

$$
t_{\text {max }}=\log \left(\frac{E}{E_{C}}\right)+C
$$

where $E$ is the energy of the incident particle and $C=-0.5$ for electron-induced cascades and $C=0.5$ for photon-induced cascades, this being the main difference between showers initiated by electrons and $\gamma$-rays.

\subsubsection{Interaction of $\gamma$-rays with Radiation Fields}

One of the fundamental reactions involving $\gamma$-rays consists of two photons interacting with each other and producing an electron-positron pair.

$$
\gamma+\gamma \rightarrow e^{+}+e^{-}
$$

From QED it is known that pair production by inelastic photon scattering has a total cross section $\sigma$ given by ${ }^{4}[81]$ :

$$
\sigma(q)=\frac{3}{8} \sigma_{T} f(q)
$$

\footnotetext{
${ }^{3}$ One radiation length is also equal to the mean distance over which a high-energy electron loses all but $1 / e$ of its energy by bremsstrahlung radiation.

${ }^{4}$ The text follows the cross section parametrization given in [271].
} 


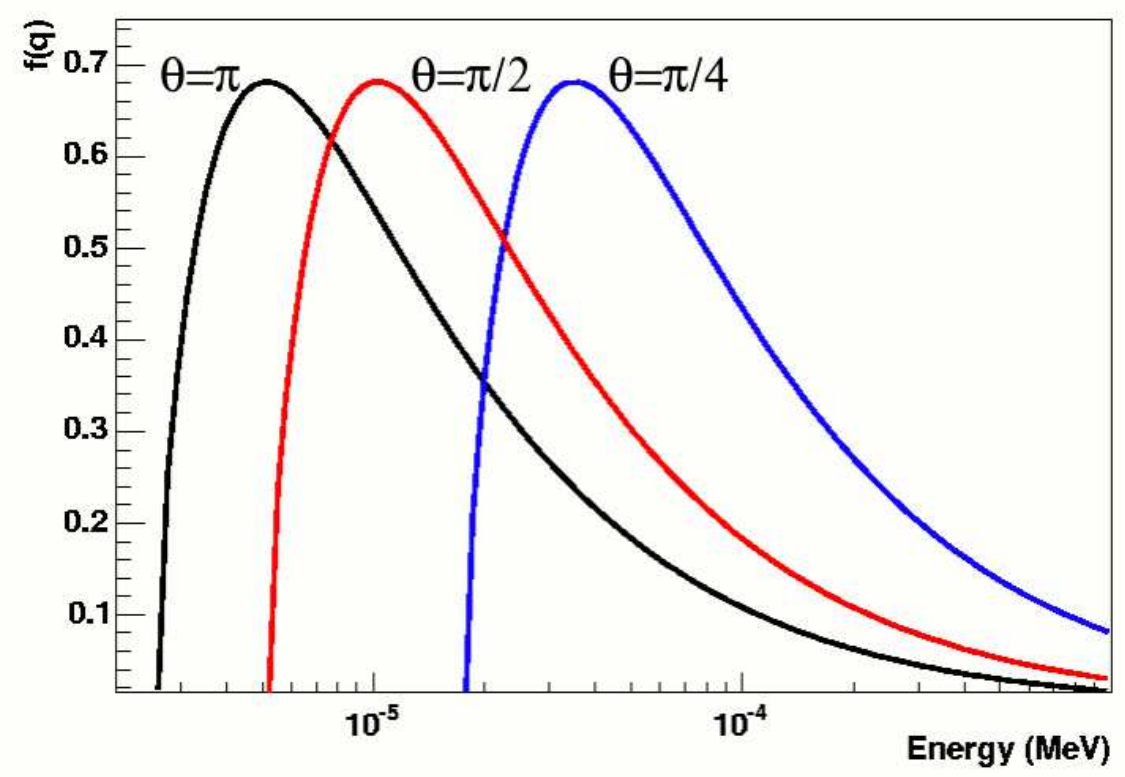

Figure 1.2: Behavior of $\mathrm{f}(\mathrm{q})$ as a function of the energy of the low energy photon for a $\gamma$-ray with energy $100 \mathrm{GeV}$. $\mathrm{f}(\mathrm{q})$ for angles $\theta=\pi, \pi / 2, \pi / 4$ are shown.

with

$$
f(q)=q\left[\left(1+q-\frac{q^{2}}{2}\right) \ln \frac{1+\sqrt{1-q}}{1-\sqrt{1-q}}-(1+q) \sqrt{1-q}\right]
$$

and

$$
q=\frac{2 m_{e}^{2}}{E \epsilon(1-\cos \theta)}
$$

where $\sigma_{T}=6.67 \times 10^{-25} \mathrm{~cm}^{2}$ is the Thomson cross section, $m_{e}=511 \mathrm{keV}$ is the mass of the electron, $E$ and $\epsilon$ are the energies of the photons, and $\theta$ is the collision angle. Since $f(q)$ peaks strongly at $q \sim 0.5$ (see fig. 1.2 ), a $\gamma$-ray with energy $E_{\gamma}$ has an enhanced probability of being absorbed by a low energy photon with energy

$$
\epsilon \sim \frac{1 k e V}{\left(\frac{E}{1 G e V}\right)(1-\cos \theta)}
$$

Figure 1.2 shows $f(q)$ for a $\gamma$-ray photon with energy $100 \mathrm{GeV}$ as a function of $\epsilon$ for three different collision angles. For a head-on collision $(\theta=\pi)$, the cross section 
is maximized when the low-energy photon has a wavelength equal to:

$$
\lambda(\mathrm{nm})=2.41 \frac{E}{1 \mathrm{GeV}}
$$

For a $\gamma$-ray incident in an isotropic radiation field, a perfect head-on collision is rather an exceptional circumstance, and thus, in the general case, the most efficient attenuation occurs when [271]

$$
\lambda(\mathrm{nm})=1.33 \frac{E}{1 \mathrm{GeV}}
$$

For a given $\gamma$-ray energy $E$, it is also important to consider the behavior of the pairproduction cross section for low and high values of $\epsilon$. At low $\epsilon$ we have the presence of a threshold below which the reaction cannot occur because of conservation of energy. At high energy the behavior of $f(q)$ is given by

$$
\lim _{\epsilon \rightarrow \infty} f(q) \approx \lim _{q \rightarrow 0} q\left[\ln \frac{2}{q}\right]
$$

resulting in a cross section that converges slowly to zero. This indicates that a $\gamma$ ray with energy $E$ can still be effectively attenuated by photons with wavelengths significantly shorter than $\lambda(\mathrm{nm})=1.33(E / 1 \mathrm{GeV})$.

\subsubsection{Detection of Astronomical $\gamma$-rays}

Gamma-ray astronomy must overcome two important obstacles from the observational point of view:

- A single $\gamma$-ray carries the energy of $10^{6}-10^{14}$ optical photons, and thus, even the most energetic processes in the universe emit a relatively small number of $\gamma$ rays. Gamma-ray instruments deal with this by maximizing as much as possible their collection area. 
- Earth's atmosphere is highly opaque to $\gamma$-rays because of its integrated matter density $\left(\sim 1000 \mathrm{~g} \mathrm{~cm}^{-2}\right)$. leading to an electromagnetic shower every time that a $\gamma$-ray hits the atmosphere. In the case of very high energy photons ( $E \gtrsim 100 \mathrm{GeV}$ ), a significant fraction of the shower is able to reach the ground. Dealing with the atmosphere has led to two types of instruments: spaceborne detectors that go above the atmosphere and detect the $\gamma$-rays directly, and ground-based detectors that image or sample the electromagnetic shower produced by a $\gamma$-ray. Both types of instruments are described below.

\subsubsection{Detection of $\gamma$-rays in Space}

Space instruments detect the $\gamma$-rays directly by making use of some of the $\gamma$-ray interactions in matter described in sec. 1.1.1.2. The most common types of detectors are introduced below.

\section{Scintillators}

In scintillators, the secondary electron produced by a $\gamma$-ray via photoelectric absorption or Compton scattering moves through the high density material exciting electrons along the way before being stopped. After some characteristic time, the electrons will recombine with the lattice and emit light. This light output is collected by a sensor such as a photomultiplier tube (PMT) or photodiode, and the strength of the signal is used to determine the energy of the incident $\gamma$-ray.

The most desirable characteristics for a scintillator are: high density (to convert many $\gamma$-rays), high light output (to determine the $\gamma$-ray energy), index of refraction close to that of glass (if coupling to a photomultiplier tube), and quick timing properties (pulse-rise time and afterglow). Some of the most common crystals (with impurities in parenthesis) are $\mathrm{NaI}(\mathrm{Tl}), \mathrm{CsI}(\mathrm{Tl}), \mathrm{CsI}(\mathrm{Na})$ and $\mathrm{Bi}_{4}\left(\mathrm{GeO}_{4}\right)_{3}$, better known as BGO. 


\section{Pair-Conversion Detectors}

For energies above $\sim 20 \mathrm{MeV}$, the dominant interaction of $\gamma$-rays in matter is pair production. Hence, pair-conversion detectors use a high-Z material like Tungsten or Lead to facilitate the conversion of $\gamma$-rays into electron-positron pairs, whose energy and direction can be measured as they pass through the detector system. Since the Large Area Telescope (LAT) on board the Gamma-ray Large Area Space Telescope (GLAST) is a pair conversion telescope, a full explanation of pair-conversion detectors will be given in section 3.1 .

\subsubsection{Detection of $\gamma$-rays on the Ground}

\section{Cherenkov Radiation Detectors}

When a charged particle propagates through a medium it disrupts the electrons in the material. After the particle passes by, these electrons will go back to equilibrium and in normal circumstances this response will be incoherent and no macroscopic effect will be observed. However, when such disruption travels faster than the speed of light in that medium, the response from the medium will be coherent and a intense flash of radiation will be produced. This is known as Cherenkov radiation [122]. This radiation is emitted in a cone with opening angle that depends on the velocity of the incident particle and the index of refraction of the medium.

Cherenkov radiation is relevant in gamma-ray astrophysics when $\gamma$-rays with energy above $\gtrsim 100 \mathrm{GeV}$ hit the atmosphere. The particles produced in the subsequent electromagnetic shower are very energetic and thus travel faster than the speed of light in air, therefore producing Cherenkov radiation. Typically the flash cone has an opening angle of $\sim 1^{\circ}$, lasts for a few nanoseconds, and peaks in the blue to near-UV [157]. The detection principle of Cherenkov detectors is to collect as much as possible of this radiation with optical mirrors (see fig. 1.3) and then use the intensity and 
direction of the flash to measure the energy and direction of the $\gamma$-ray.

To distinguish the Cherenkov light flashes originated by $\gamma$-rays from the ones originated by cosmic rays (mostly protons) two techniques have been developed. The first technique samples the wave front at different places of the radiation cone and measures its timing properties and intensity profile to separate showers created by $\gamma$-rays from those created by cosmic rays. Experiments like STACEE [105] and CELESTE [55] use this technique. The second technique is known as the imaging technique. In this case, the mirrors focus the light in a camera made of PMTs obtaining a pattern for every shower. The shape, intensity and timing of the pattern are used to separate the $\gamma$-ray events from the cosmic-ray showers and other background from local, passing-by muons. Imaging Atmospheric Cherenkov Telescopes (IACTs) have a small field of view (limited by optical aberration and cost) and relatively low duty cycles (observing time is restricted to moonless, clear nights). This is compensated however, with enormous collection area and superb angular resolution, which allows IACTs to study the very short-term variability and spatial structure (point-like or

extended aspect) of many $\gamma$-ray sources. Current IACTs include HESS [111], MAGIC [51], VERITAS [112], and CANGAROO [205].

\section{Extensive Air-Shower Arrays}

Instead of detecting the Cherenkov light produced in the atmosphere by the electromagnetic cascade, an Extensive Air-Shower (EAS) array like Milagro [245] detects the particles in the shower (see fig. 1.3). The direction of the original $\gamma$-ray can be calculated from the timing of the arrival particles in the array, and the energy is directly related to the number of detected particles. These detectors must be huge to sample enough of the shower, and ideally they are located at high altitude so that they can catch the shower before it loses too much energy in the atmosphere. Extensive air-shower arrays only sample the fraction of the shower that reaches the ground, 


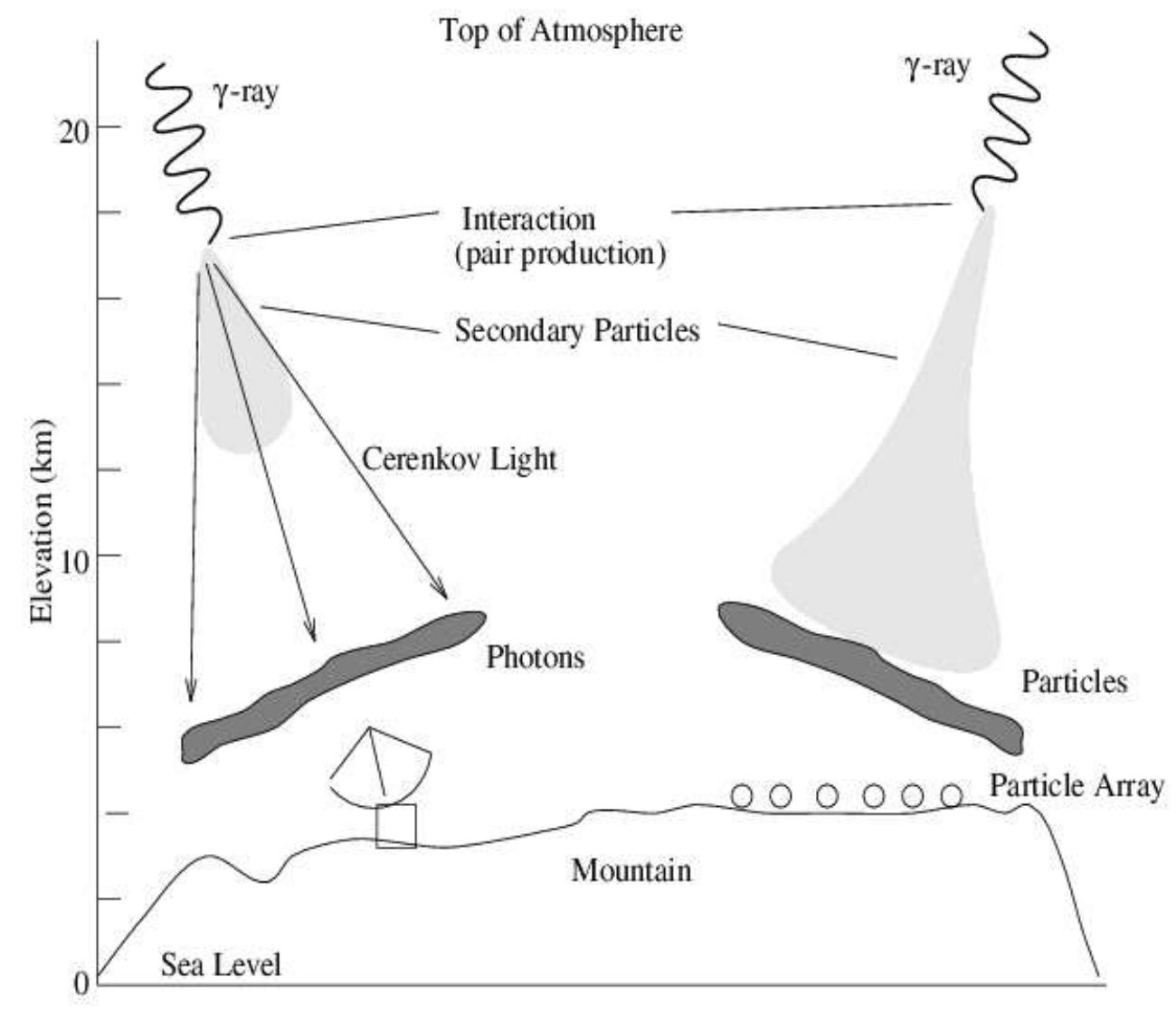

Figure 1.3: Schematic representation of $\gamma$-ray induced showers (not to scale) and their detectors. The IACT on the left detects the Cherenkov radiation emitted by the particles in the cascade. The Extensive-air-shower array on the right detects the secondary particles directly. Adapted from [153]. 
and thus, shower imaging with this type of instrument is difficult and sensitive to shower fluctuations. This leads to modest energy and angular resolution. Nevertheless, extensive air-shower arrays can operate during day and night, and have a large field of view, making them very suitable for all-sky surveys.

\subsubsection{The Legacy of CGRO and EGRET}

The Compton Gamma-ray Observatory (CGRO, 1991-2000) was one of NASA's great observatories. CGRO had four instruments (fig. 1.4) that covered an unprecedented six decades of the gamma-ray sky from $30 \mathrm{keV}$ to $30 \mathrm{GeV}$. These instruments were the Burst And Transient Source Experiment (BATSE) [84], the Oriented Scintillation Spectrometer Experiment (OSSE) [129], the Imaging Compton Telescope (COMPTEL) [241], and the Energetic Gamma Ray Experiment Telescope (EGRET) [262].

BATSE was a gamma-ray burst (GRB) detector that contributed thousands of GRB locations and lightcurves. BATSE's results led to the distinction between short and long GRBs and to the proposal of theoretical models to explain the origin and working principle of these huge gamma-ray explosions. Furthermore, BATSE revealed that GRBs are distributed isotropically in the sky. This breakthrough observation supported the extragalactic-origin hypothesis for GRBs, which was confirmed in 1997 [220] with the first GRB redshift determination (from optical afterglow). COMPTEL and OSSE with their spectroscopic capabilities revealed the presence of isotopes and annihilation lines in the galactic center and in the solar flare of 1991.

EGRET was a pair-conversion telescope that studied the $\gamma$-ray sky between 20 $\mathrm{MeV}$ and $\sim 30 \mathrm{GeV}$. It had a medium-size field of view (approximately a gaussian shape with a half width at half maximum of about $20^{\circ}$ ), its effective area was 1500 $\mathrm{cm}^{2}$ between $200 \mathrm{MeV}$ and $1000 \mathrm{MeV}$ (falling at higher and lower energies), and a point spread function that depended strongly on the energy $\left(4.3^{\circ}\right.$ at $35 \mathrm{MeV}$ down to $0.4^{\circ}$ at $2 \mathrm{GeV}$ ) [201]. Beyond the instrumental and technical advances obtained with 


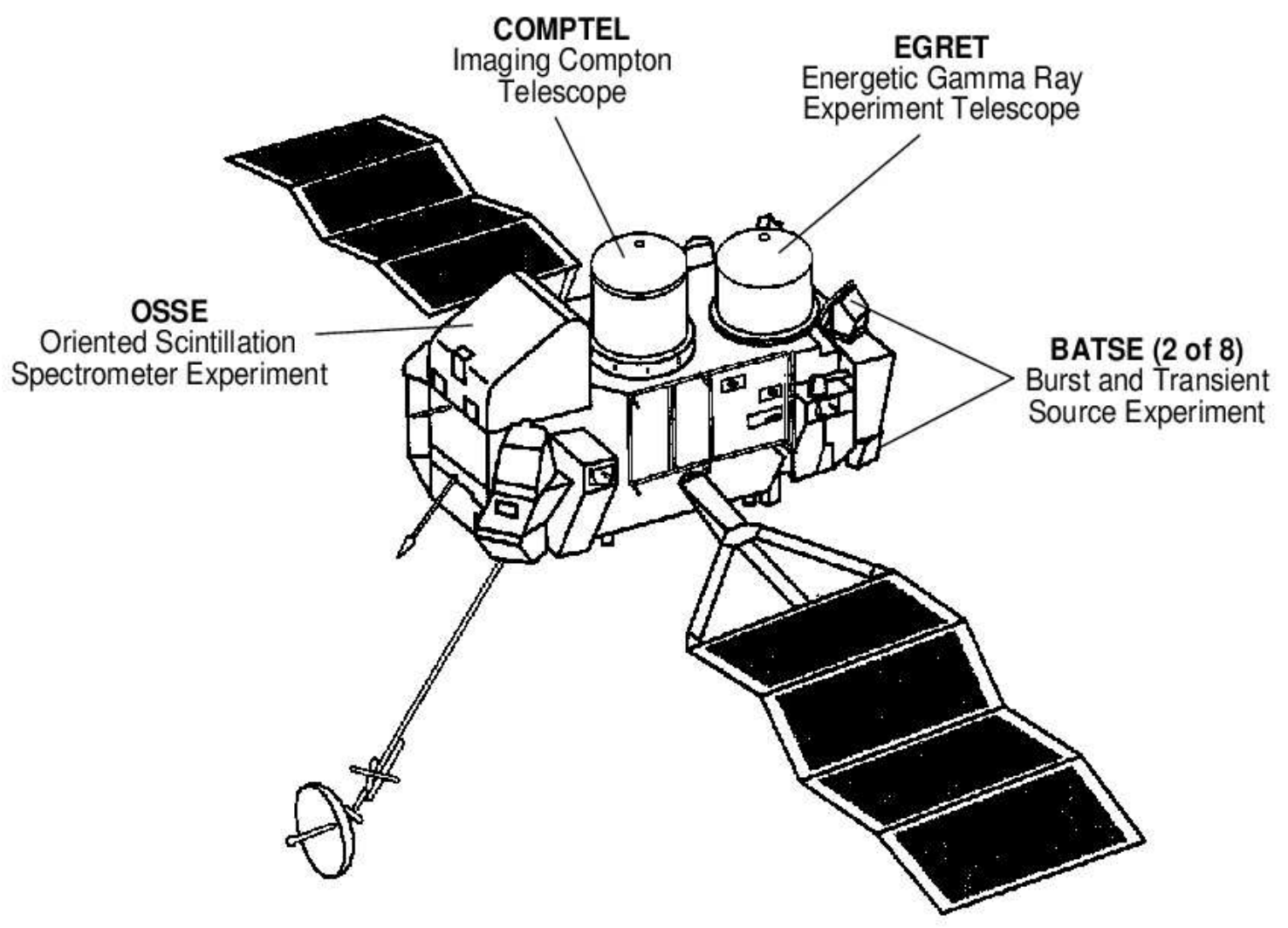

Figure 1.4: The instruments on-board the Compton Gamma-ray Observatory (CGRO). 
EGRET, its true legacy is the new and exciting picture of the gamma-ray universe that it presented. EGRET made both expected and unexpected discoveries that revolutionized the field of high-energy astronomy. It confirmed that the galaxy is a strong emitter of $\gamma$-rays because of the interaction of cosmic rays with the interstellar gas, discovered that some pulsars are copious emitters of $\gamma$-rays, and most important, it established blazars as the largest class of extragalactic $\gamma$-ray sources.

\subsection{Gamma-ray Emission of Blazars}

The research in this dissertation describes how to use the blazars that GLAST (described in Chapter 3) will observe as a tool to probe the cosmic background radiation known as EBL (Extragalactic Background Light; described in Chapter 2). A discussion of blazars and their $\gamma$-ray emission is thus necessary to explore the potential of blazars as a probe of the EBL.

\subsubsection{AGN Properties and Definition}

In a small fraction $(\lesssim 10 \%)$ of the observable galaxies in the universe, the central core seems to outshine all the stars in the galaxy. When studying their spectrum, these core-dominated galaxies often present broad emission lines (see fig. 1.5) instead of the absorption lines that characterize most galaxies. In addition, the spectrum of these core-dominated objects extends over a large range of wavelengths with maxima in IR, UV, X-rays or even $\gamma$-rays. More importantly, these galaxies seem to be dominated by a powerful non-thermal emission while normal galaxies radiate most of their energy thermally in the UV to infrared band, a result of the combined light output of the constituent stars.

From the dynamical point of view, these core-dominated galaxies are highly variable, with significant flux changes on time scales of days or even less, hence the name 


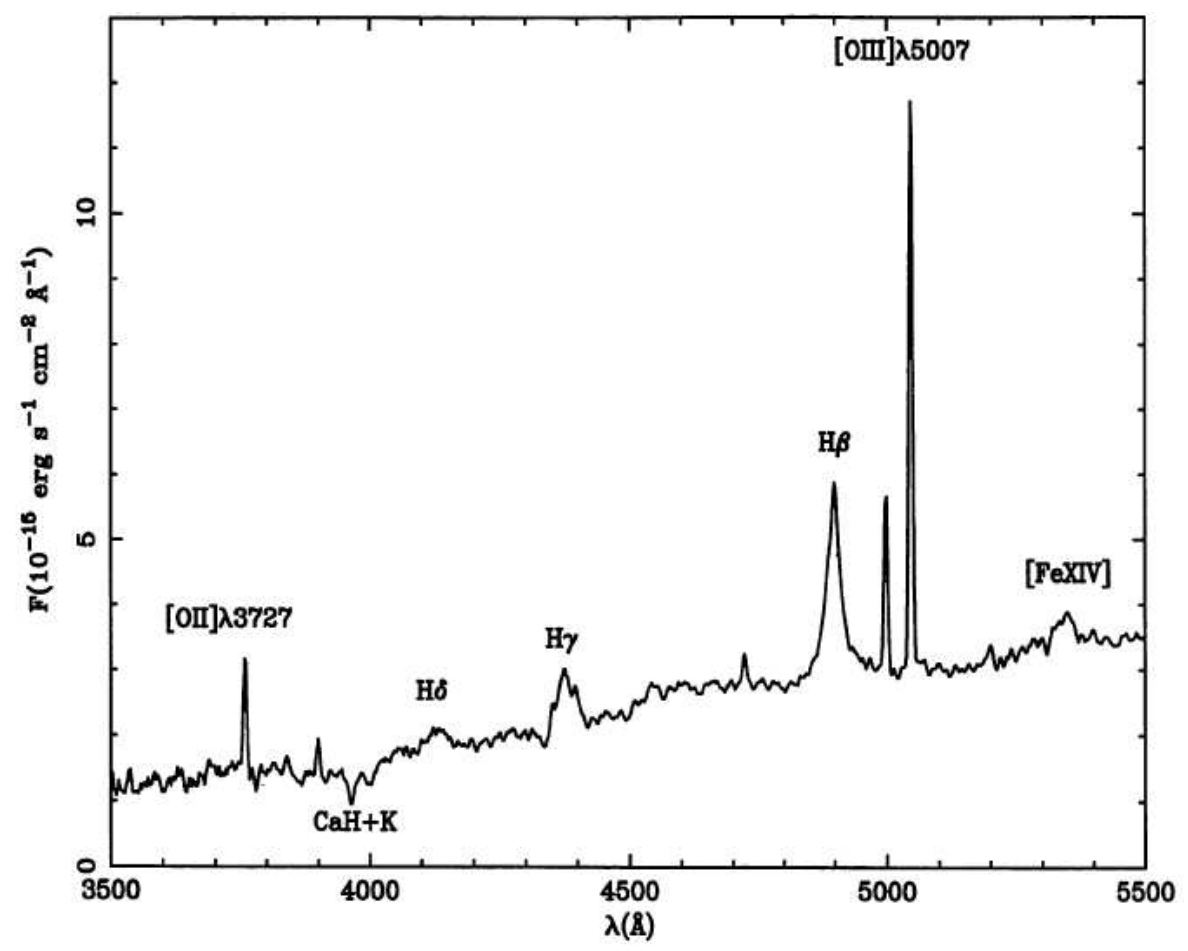

Figure 1.5: The blue band optical spectra of the Seyfert type 1 galaxy MGC-6-30-15, from [229].

Active Galactic Nuclei (AGN).

From the few properties outlined above, it is possible to learn a lot about these objects from basic physical principles [48]. From the short-term variability $\Delta t$ and using light-travel arguments, the size of the emission region $r$ can be constrained to

$$
r<c \frac{\Delta t}{(1+z)}
$$

where $c$ is the speed of light and $z$ is the redshift of the source. This implies that for a source at redshift $z=1$, and variability scale of 1 day (as it has been observed), the size of the emission region is of the order $1.3 \times 10^{10} \mathrm{~km}$, roughly the size of the Solar System. The distance to a typical source $(z \sim 1)$, combined with an average magnitude in the optical band, amounts to a luminosity in the order of $10^{46} \mathrm{erg} \mathrm{s}^{-1}$ which is larger than the most luminous normal galaxies. 
Since nuclear burning in stars is not able to release such amount of power in such small space, the only known alternative that makes sense is the conversion of gravitational energy into radiation by accretion, which is the most efficient way known to transform rest-mass energy into radiation. The luminosity of an accretion process is proportional to the mass accretion rate $\dot{m}_{a c c}$ [48],

$$
L_{a c c}=\epsilon \dot{m}_{a c c} c^{2}
$$

where $\epsilon$ is a parameter describing the efficiency of the process $(\epsilon \sim 10 \%)$. During accretion, conservation of angular momentum dictates that the in-falling mass will swirl around the central mass with increasing speed at the same time that it heats up due to its viscosity. This thermal energy is radiated away as infrared, optical, $\mathrm{UV}$ and X-ray radiation. If the radiation becomes too intense, the radiation pressure could eventually counter-balance the gravitational pull and stop the accretion process altogether. This equilibrium condition where the radiation pressure cancels exactly the gravitational pull is known as the Eddington limit [48]. The maximum luminosity that is below the limit is given by:

$$
L_{E d d} \sim 10^{38} \frac{M}{M_{\odot}}\left(\operatorname{erg~s}^{-1}\right)
$$

Thus, the source described above with luminosity $10^{46} \mathrm{erg} \mathrm{s}^{-1}$ must have a mass above $10^{8}$ solar masses in order to satisfy the Eddington limit. Even if a non-spherical symmetry is assumed and the limit above is relaxed, only a supermassive black hole can have such a large mass in a volume that is not that much bigger than the Solar System. 


\subsubsection{AGN Taxonomy}

AGN are usually divided in classes and subclasses that are more based on historical developments and detection biases than in fundamental properties. In consequence, categories like quasars, QSOs, Seyferts, BL Lacs, and others, frequently overlap. It follows then that this taxonomic nomenclature although useful and widespread is purely observational and does not necessarily provide insight into the underlying similarities and differences of the astrophysical objects. The most important AGN classes are introduced here for reference.

\section{Seyfert Galaxies}

Like other AGN, Seyfert galaxies contain a very bright nucleus, but in this case the host galaxy is also detectable. The original definition of the class was based on its morphology, but subsequent spectroscopical observations discovered unusual (for a galaxy) emission-line characteristics, as illustrated in fig. 1.5. The presence or absence of broad emission lines gave origin to two distinct subclasses of Seyfert galaxies: Type 1 which have both narrow and broad emission lines, and Type 2 which only show narrow lines [218].

\section{QSOs}

Quasi Stellar Objects (QSOs) appear unresolved during sky survey observations (i.e. star-like morphology) and comprise the most luminous class of AGN ( $\left.L \gtrsim 10^{44} \mathrm{erg} \mathrm{s}^{-1}\right)$. QSO spectra are remarkably similar to those of Seyfert galaxies, except that the narrow lines are generally weaker [218]. According to their radio flux, QSOs are further classified into quasars if $L_{5 \mathrm{GHz}} \gtrsim 10^{24.7} \mathrm{~W} \mathrm{~Hz}^{-1} \mathrm{sr}^{-1}$, and radio-quiet QSOs otherwise. 


\section{Quasars}

Quasars represent a small minority $(\sim 5-10 \%)$ of the AGN population [218]. According to their radio emission as a function of frequency $\nu$, they are subdivided into Flat Spectrum Radio Quasars (FSRQs), with $F_{\text {radio }} \propto \nu^{\alpha},(\alpha>-0.5)$, and Steep Spectrum Radio Quasars (SSRQs) with $\alpha<-0.5$. FSRQs are radio-bright in a compact region, while SSRQs present an extended radio-emitting region.

\section{Radio Galaxies}

Radio galaxies are nearby resolved objects that have optical spectra of the sort associated with AGN [218]. They are subdivided in Narrow Line Radio Galaxies (NLRGs) and Broad Line Radio Galaxies (BLRGs) that are analogous to type 2 and 1 Seyfert galaxies. According to their morphology, radio galaxies can be further subdivided into Fanaroff-Riley type 1 (FR1) galaxies, with a low radio luminosity $\left(\mathrm{L}_{r}<5 \times 10^{41} \mathrm{erg}\right.$ $\mathrm{s}^{-1}$ ) that is maximum at the core; and FR type 2 (FR2) galaxies that are radio-bright and present hot spots in the form of lobes separated from the nucleus (as illustrated in fig. 1.6). Strong jets are seen to emanate perpendicular to the accretion disk and because of its power-law spectra and high degree of linear polarization are generally thought to result from the synchrotron emission of relativistic particles. Although radio emission from galaxies is strongly associated with the presence of jets, it is not known how these jets form, what are they made of, and how they are collimated over vast distances. It is clear that jets unambiguously connect the active galactic nucleus to the galaxy exterior, and therefore, they represent the only known mean of energy transport. Furthermore, the detection of apparent superluminal motion by very long baseline interferometry [130] provides clear evidence of relativistic bulk motion, and thus, extreme plasma acceleration and high energy non-thermal emission (including $\gamma$-rays in the case of blazars) are believed to take place in jets. In the next section AGN jets will be considered again in the context of gamma-ray emission. 

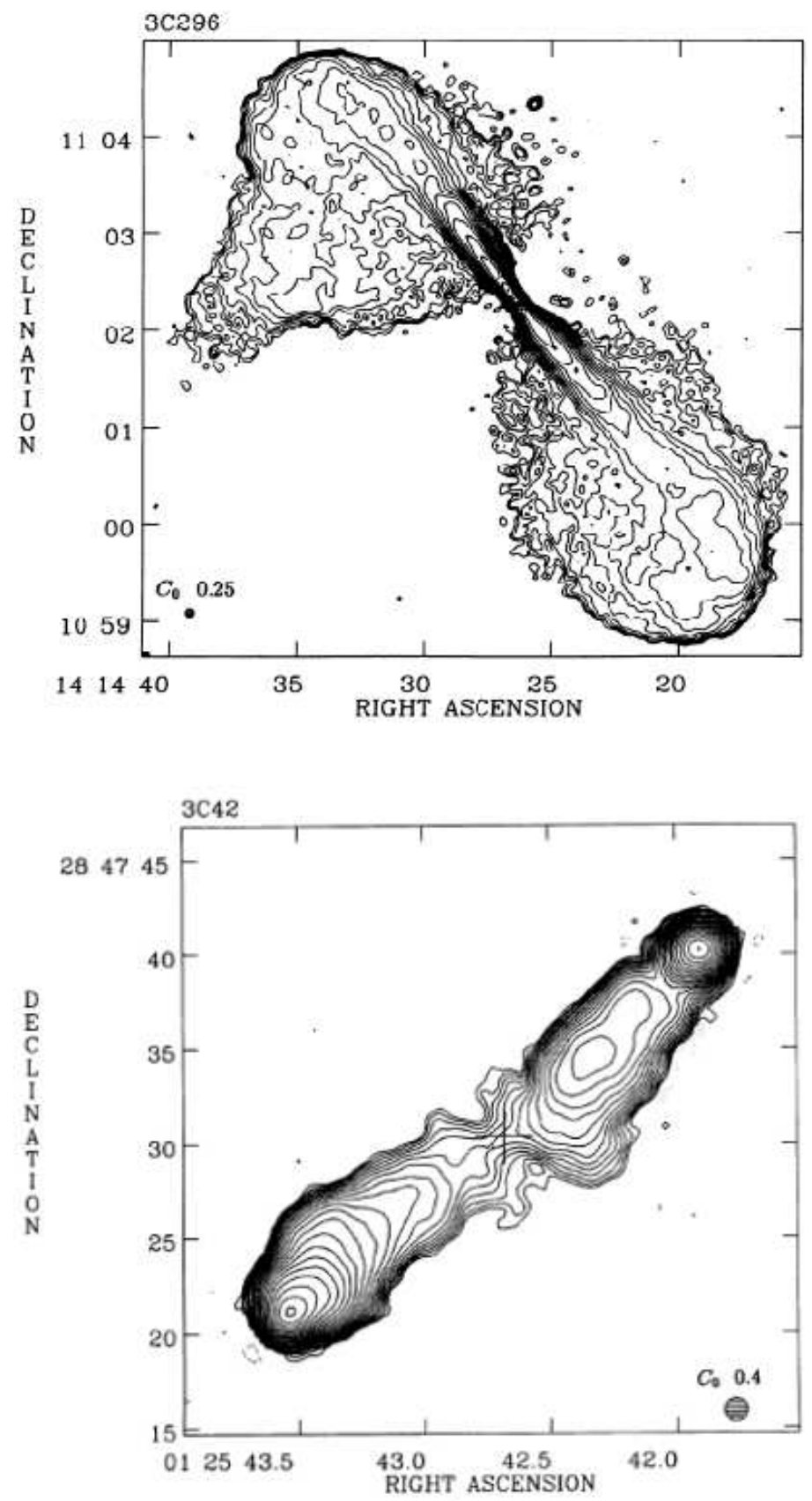

Figure 1.6: Radio intensity maps for two radio galaxies. Radio galaxy 3C296 at the top has maximum radio emission at the core and therefore is classified as FR1. For comparison, a FR2-type galaxy $(3 \mathrm{C} 42)$ is shown in the bottom map. Adapted from [155]. 


\section{Blazars}

Blazars are radio-loud AGN with core-dominated emission. They display a flat radio spectrum (as defined above), high and variable optical polarization, and rapid flux variability at all wavelengths from radio to $\gamma$-rays. The blazar class is composed of BL Lac type objects and FSRQs. BL Lacs are not considered quasars because of their nearly featureless optical continuum (i.e. absent or extremely weak emission lines). As a result, BL Lacs are extremely hard to find in optical surveys and their discovery is due to radio or X-ray observations. Blazars are the most extreme subset in the AGN population: they are the brightest, the most variable and the most energetic, reaching photon energies up to the $\mathrm{TeV}$ regime. Thanks to EGRET it is now known that this type of objects dominate the extragalactic $\gamma$-ray sky.

\subsubsection{The AGN Paradigm}

Attempts have been made to explain the different classes of AGN as different manifestations of the same astronomical population with a common underlying astrophysical origin [270]. In order to gain understanding of the relationship among different classes, AGN have been classified according to two observational features: radio-loudness and optical emission lines. Radio-loudness relates to the strength of the radio emission compared to the optical flux. Specifically, an AGN is radio-loud if

$$
\frac{f_{5 \mathrm{GHz}}}{f_{\mathrm{B}}}>10
$$

where $f_{5 \mathrm{GHz}}$ is the radio flux at $5 \mathrm{GHz}$ and $f_{\mathrm{B}}$ is the flux in the $\mathrm{B}$ optical band. Applying this distinction, radio-loud AGN represent roughly $\sim 10-20 \%$ of the total ${ }^{5}$. Following the classification of Seyfert and radio galaxies (from their emission line

\footnotetext{
${ }^{5} \mathrm{~A}$ recent study [126] finds that the fraction of radio-loud AGN is a strong function of stellar mass in the galaxy or central black hole mass: the fraction increases from zero at a stellar mass of $10^{10} M_{\odot}$ to $30 \%$ at a stellar mass of $5 \times 10^{11} M_{\odot}$
} 


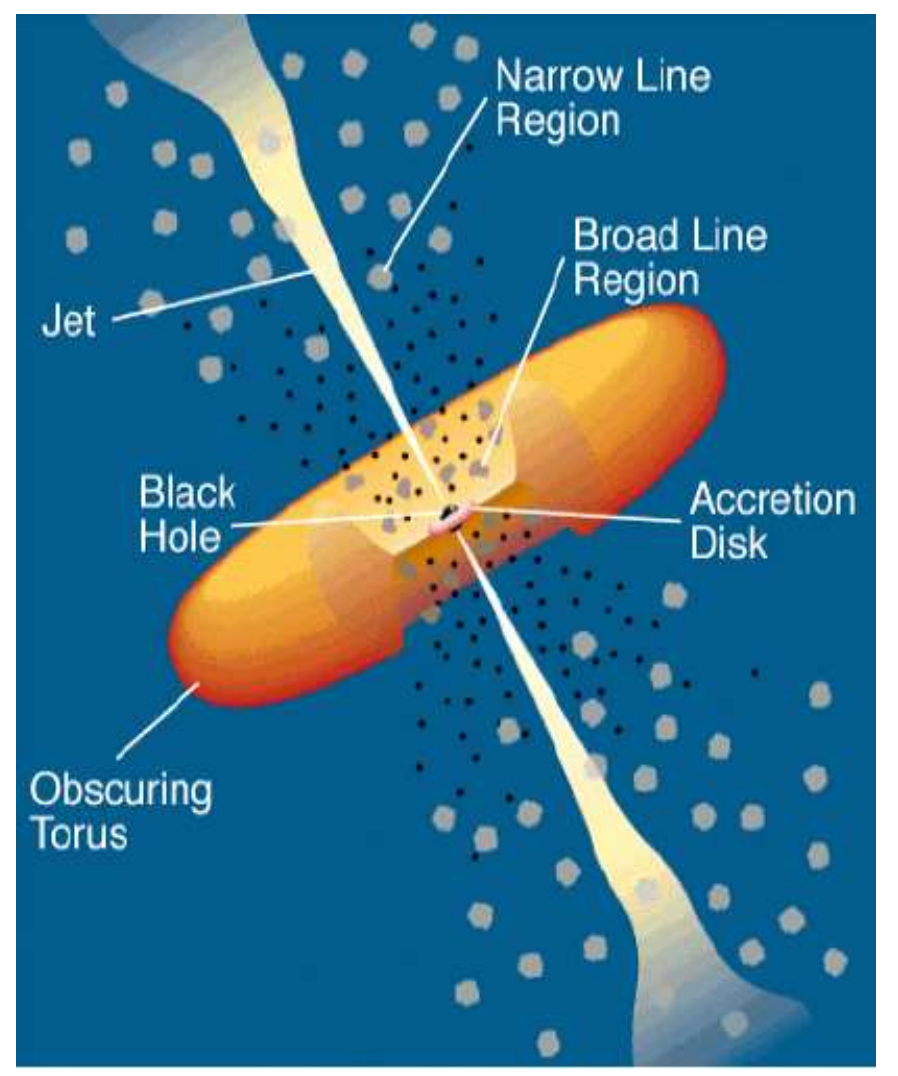

Figure 1.7: Adapted from [270]. The radio-loud AGN paradigm: A supermassive black hole at the center of the galaxy surrounded by an accretion disk and an obscuring torus. The inner molecular clouds (dark spots) move within the torus at high velocities and are responsible for the broad emission lines. Narrow lines (gray spots) are emitted by slow-moving clouds outside the torus. The dark spots represent a hot electron corona that scatter light from the disk and the broad-line region. A strong jet of relativistic particles is emitted perpendicular to the accretion disk. The AGN model for radio-quiet $\mathrm{AGN}$ is equivalent, with the fundamental difference that no jets are present. 


\begin{tabular}{|c|c|c|c|}
\hline Radio Loudness & $\begin{array}{c}\text { Type } 0 \\
\text { (narrow lines) }\end{array}$ & $\begin{array}{c}\text { Type } 1 \\
\text { (broad lines) }\end{array}$ & $\begin{array}{c}\text { Type2 } \\
\text { (weak/absent) }\end{array}$ \\
\hline Radio-quiet & Seyfert 2 & $\begin{array}{c}\text { Seyfert } 1 \\
\text { QSO }\end{array}$ & \\
\hline Radio-loud & $\begin{array}{c}\text { NLRG } \\
(\text { FR I, FR II) }\end{array}$ & $\begin{array}{l}\text { BLRG } \\
\text { SSRQ } \\
\text { FSRQ }\end{array}$ & $\begin{array}{c}\text { Blazars } \\
\text { (BL Lac, FSRQ) }\end{array}$ \\
\hline
\end{tabular}

Table 1.1: Adapted from [48]. Classification of AGN according to their radio-loudness and optical emission lines. QSO: Quasi Stellar Objects; NLRG, BLRG: Narrow and Broad Line Radio Galaxies; FR I, FR II: Fanaroff-Riley type I and II radio galaxies; SSRQ, FSRQ: Steep and Flat Spectrum Radio Quasars.

properties), AGN in general are divided into Type 0 sources, with weak or absent lines, Type 1 sources, showing both narrow and broad emission lines, and finally, Type 2 sources with narrow lines only. This two-dimensional simple classification scheme is shown in Table 1.1.

The AGN paradigm (see fig. 1.7) consists of a supermassive black hole $\left(\sim 10^{6}\right.$ - $10^{10} \mathrm{M}_{\odot}$ ) at the center of the galaxy with a Schwarzschild radius of the order of $10^{-5} \mathrm{pc}\left(1\right.$ parsec $\left.=3.086 \times 10^{16} \mathrm{~m}\right)$. The black hole is surrounded by an accretion disk of ionized plasma that is responsible for the optical continuum emission. In turn, the accretion disk is surrounded by an external torus of obscuring molecular gas that extends up to $\sim 30$ pc. Within the torus, fast-moving molecular clouds are ionized by the radiation from the accretion disk (dark spots in fig. 1.7). These clouds are responsible for the observed broad emission lines. Outside the torus, the molecular clouds move with lower speeds $\left(v<2000 \mathrm{~km} \mathrm{~s}^{-1}\right)$, resulting in narrow emission lines (gray spots). The dark dots in fig. 1.7 represent a hot electron corona in the inner region that is responsible for the scattering of continuum and broad line emission that has been observed in some Seyfert type 2 galaxies. The presence of a strong jet that propagates perpendicular to the accretion disk is characteristic of radio-loud AGN.

Following the AGN model introduced above, the observational properties of a given source are thought to be largely determined by orientation effects, i.e. they 
depend on the observation angle. When looking down the jet of a radio-loud AGN, both the collimated emission from the jet and the unobscured radiation from the disk are observed. In this case, the source consists of the superposition of continuum emission from the disk, broad and narrow-line emission ${ }^{6}$ from the molecular clouds, and superluminal Doppler boosted radiation from the jet (including the characteristic flat radio spectrum emission); all properties of the blazar class of AGN.

At offset angles of $\sim 30^{\circ}$ the collimated emission from the jet is not observed anymore, however there is still an unobscured view of the central region. Narrow and broad lines can be observed, combined with a steep radio spectrum. This is the case for SSRQs, BLRGs (if radio-loud) and Seyfert type 1 galaxies (if radio-quiet). At larger observation angles, the broad-emission lines are obscured by the molecular torus and only the narrow emission lines are observed (Seyfert type 2, NLRGs). In the case of a radio galaxy, two strong oppositely aligned jets are observed.

\subsubsection{Gamma-ray Emission}

\subsubsection{Observations}

\section{The EGRET years}

As introduced in section 1.1.3, EGRET established blazars as the largest population of extragalactic $\gamma$-ray sources. The Third Egret Catalog [106] contains 66 highconfidence identified blazars with another 27 low-confidence identifications detected in the energy range $100 \mathrm{MeV}$ to $\sim 30 \mathrm{GeV}$ (sec. 5.4.2.1). The energy spectra of the blazars observed by EGRET can be well characterized by power laws with an average photon spectral index $(\alpha)$ of $2.15 \pm 0.04$ [196]

\footnotetext{
${ }^{6} \mathrm{It}$ is believed that the molecular clouds responsible for emission lines are not present in BL Lac objects. Therefore no emission lines are observed even in the case of unobscured view of the central region of the AGN (observation down the jet).
} 


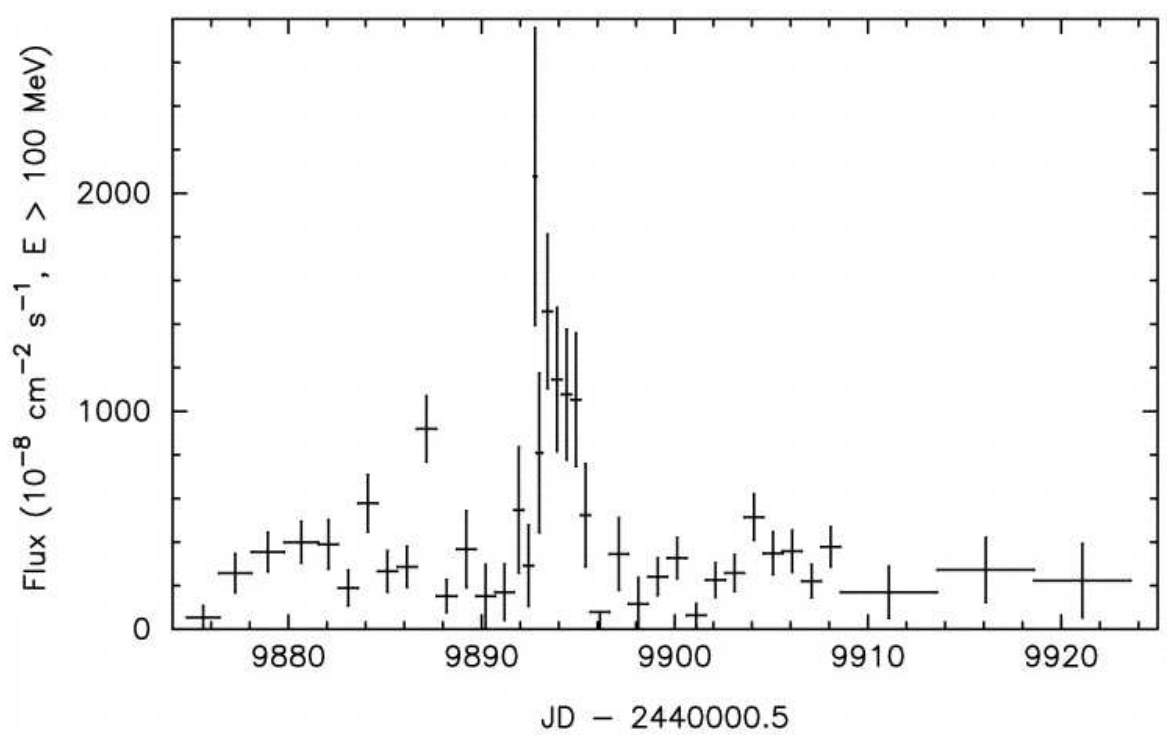

Figure 1.8: From [181]. EGRET light curve for PKS 1622-297 as an example of the variability of blazars. The vertical bars indicate the ranges of the $68 \%$ confidence flux estimates.

$$
F(E)=k\left(\frac{E}{E_{0}}\right)^{-\alpha} \text { photons } \mathrm{cm}^{-2} \mathrm{~s}^{-1} \mathrm{MeV}^{-1}
$$

Short-term variability is one of the most notable features in the $\gamma$-ray emission of blazars. The only sources where EGRET did not observe variability were the faintest ones, just above the detection limit. For example, flux variations by a factor of 80 were observed during a major flare of PKS 1622-297 [181] with doubling time scales of a few hours (see fig. 1.8).

\section{TeV Emission from Blazars}

Thanks to the significant improvements in detector sensitivity that have been achieved by Atmospheric Cherenkov Telescopes (sec. 1.1.2.2), the discovery of TeV emission from extragalactic sources has become frequent. Over twelve blazars have been now reported to emit (at least occasionally) in the $\sim>300 \mathrm{GeV}$ range. These sources are all members of the BL Lac subclass of blazars, and because of EBL absorption (sec. 2.6.4), they are usually among the closest of the class (redshift $\ll 1$ ). In common 
with EGRET observations, one of the most striking features of $\mathrm{TeV} \gamma$-ray emission from AGN is their variability.

An important recurrence that should not be overlooked is that, to date, most of the EGRET blazars have not been detected by TeV telescopes; even those that are nearby and bright. This indicates a spectral break or rolloff taking place between the EGRET energy range and the $\mathrm{TeV}$ regime. Furthermore, most of the detected $\mathrm{TeV}$ blazars belong to the same subset, namely, the X-ray selected BL Lac (XBL) objects, that represent only a small fraction of the sources seen with EGRET. This suggests a rolloff for non-XBL blazars. Finally, the few blazars that have been seen in GeV and $\mathrm{TeV}$ energies have $\mathrm{TeV}$ fluxes that are lower than expected from a simple power law. Unfortunately, with little observational data in the $10-300 \mathrm{GeV}$ range, no firm conclusions can be drawn about the precise shape of the spectra at such energies. This is one of the main motivations for the next generation of ground based experiments and GLAST.

\subsubsection{Spectral Energy Distributions and the Blazar Sequence}

The Spectral Energy Distribution (SED) of blazars is characterized by the broad range of energy emission illustrated in fig. 1.9. SED plots usually present the radiated power per logarithmic frequency interval, so that the released energy in the different wavelength bands can be compared directly. A typical blazar-spectrum shows two pronounced peaks, which suggests at least two different emission components and processes. The first bump is interpreted as polarized synchrotron emission from relativistic electrons, while no definite explanation exists for the second bump, which accounts for the high energy emission observed in blazars (different theoretical models will be discussed in the next section). It should be noted that the high-energy emission component dominates the bolometric luminosity of the object, a powerful demonstration of the extreme nature of these sources. 
Over time, the historical distinction between radio-selected BL Lacs (RBLs) and $x$-ray selected BL Lacs (XBLs) based on detection method has been spoiled by objects like Mrk 501 that qualify both as RBL and XBL. A different classification was proposed by Padovani and Giommi [213], which divides BL Lacs depending on where the synchrotron break occurs. According to this scheme BL Lacs are either high-peaked BL Lacs (HBLs) or low-peaked BL Lacs (LBLs). It turns out in general, that RBLs are LBLs (with exceptions like Mrk 501) and XBLs are HBLs (again, with exceptions like OJ287). By looking at fig. 1.9 it is possible to realize that the X-ray emission for LBLs (RBLs) and HBLs (XBLs) has a different origin. In HBLs the X-ray radiation is produced at the high-frequency end of the synchrotron radiation; while for LBLs, the X-rays constitute the low frequency tail of the high-energy bump.

The average SEDs for FSRQs, RBLs and XBLs are shown in fig. 1.9. A few generalizations can be drawn from the plot:

- The synchrotron and high-energy peaks move sequentially to higher energies according to $\mathrm{FSRQ} \rightarrow \mathrm{RBL} \rightarrow \mathrm{XBL}$

- The frequencies for the synchrotron and high-energy peaks are proportional.

- The luminosity of the two bumps seem to be correlated.

Sambruna et al [237] analyzed the the multi-wavelength spectra of complete samples of the three kinds of blazars and concluded that the observed differences in SEDs cannot be accounted by orientation effects. Instead, smooth changes of the intrinsic physical parameters of the emitting region are required (magnetic field intensity, electron and external photon densities, etc.). This continuous transition from FSRQs to HBLs is known as the blazar sequence.

In particular, the blazar sequence predicts that the synchrotron peak frequency is anticorrelated with the blazar radio luminosity. This has strong implications for 


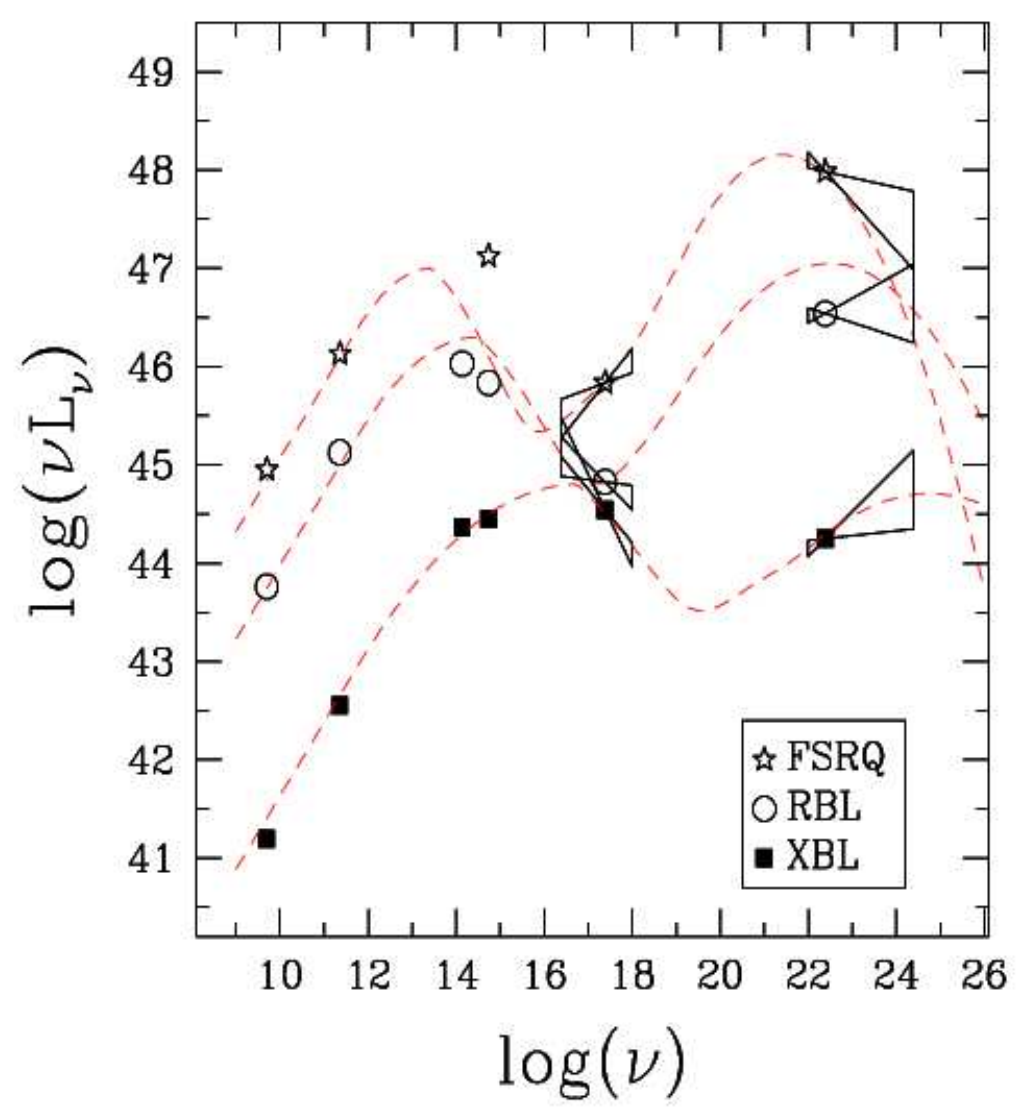

Figure 1.9: From [170]. Average spectral energy distributions of FSRQs, RBLs and XBLs as an illustration of the hypothesized blazar sequence.

the understanding of jet physics and the possible detection of high-peaked FSRQs (i.e. powerful FSRQs emitting $\gamma$-rays at $\mathrm{TeV}$ energies). Recently, blazar surveys have found outliers to this sequence, and therefore, the blazar sequence seems to be ruled out in its simplest form ([212] and references therein). Nevertheless, the maximum synchrotron peak frequency of known FSRQs appears to be $\sim 10-100$ times smaller than that typical of BL Lacs. This could be related to the jet physics, or could still be due to selection biases. 


\subsubsection{Gamma-ray Emission in Blazars}

The short-term variability and the huge inferred $\gamma$-ray luminosities of blazars suggest a highly compact and photon-dense emitting region. However, if the photon density is above a certain threshold value, the photons cannot escape because they interact via pair production (as described in section 1.1.1.3).

The internal "optical depth" for a source emitting photons above the pair-production threshold is given by

$$
\tau=n_{\gamma} \sigma R
$$

where $n_{\gamma}$ is the photon density, $\sigma$ is the cross section for pair production and $R$ is the size of the source. The photon density can be expressed in terms of the photon energy density $\left(\sim L_{\gamma} / 4 \pi R^{2} c\right)$ divided by the photon mean energy $\left(\sim>m_{e} c^{2}\right)$. Therefore, a back-of-the-envelope calculation of the optical depth for a typical blazar, with $L_{\gamma} \sim$ $10^{48} \mathrm{erg} \mathrm{s}^{-1}$, a radius of 1 light-day $\left(\sim 10^{12} \mathrm{~m}\right)$, and assuming isotropic emission, yields:

$$
\tau_{\gamma \gamma} \sim \frac{\sigma_{\gamma \gamma}}{4 \pi m_{e} c^{3}} \frac{L_{\gamma}}{R} \sim 200 \gg 1
$$

With such a large optical depth, it ought to be practically impossible to observe $\gamma$ rays from any blazar. Since large fluxes are observed nevertheless, it is accepted that the the gamma-ray emission is originated in beamed jets. Further evidence is provided by the fact that apparent superluminal motion has been observed in blazars [130], which is indicative of relativistic motion in the jets pointing at small offset angles with respect to the observer line of sight. This further supports the unification-byorientation hypothesis. A relativistic jet origin of the $\gamma$-rays has profound implications for the $\gamma-\gamma$ opacity argument, since in that case the observed gamma-radiation will be Doppler boosted:

$$
L_{\gamma}^{\text {observed }}=\delta^{n} \times L_{\gamma}^{\text {intrinsic }}
$$


where $n=3,4$ is a model-dependent factor and the Doppler factor $\delta$,

$$
\delta=\frac{1}{\Gamma(1-\beta \cos \theta)} ; \Gamma=\left(1-\beta^{2}\right)^{-1 / 2}
$$

is determined by the bulk speed $(\beta=v / c)$, and its orientation angle $(\theta)$ with respect to the line of sight. For a typical Doppler factor of 10, as it is estimated for several blazars, the actual in-situ $\gamma$-ray luminosities are a factor of $10^{3}-10^{4}$ smaller than observed. According to eq. 1.19, this translates into optical depths a factor of $10^{3}-10^{4}$ smaller than calculated for isotropic, unboosted emission. A $\gamma$-ray emitting region with $\tau<1$ is optically thin, and $\gamma$-rays can be observed far away from the source.

\section{Emission Mechanisms}

The problem of $\gamma$-ray emission in AGN has been factorized in models of i) jet formation and acceleration and ii) $\gamma$-ray production. The former try to explain the production, collimation, and acceleration of matter to relativistic speeds, while the latter tries to explain how this highly relativistic plasma produces non-thermal emission with energies all the way up to $\gamma$-rays.

Models of jet formation and particle acceleration are beyond the scope of this dissertation, nevertheless it should be mentioned that the most promising models of jet formation seem to consider magnetically driven outflows in the context of relativistic magnetohydrodynamics (MHD) [265]. Based on MHD calculations, the jet results from the extraction of rotational energy from the spinning black hole and/or accretion disk, a process that was first proposed by Blandford \& Znajek [28]. Particle acceleration within the jet is attributed to Fermi processes ([230] and references therein), where energetic particles are accelerated by repeatedly scattering off moving magnetic turbulence (or shocks). Fermi processes are thought to be responsible for 
the production of the non-thermal power-law particle distributions observed in the spectral energy distributions of AGN.

Models of $\gamma$-ray production in blazars try to reproduce the broad multi-wavelength spectra and rapid variability observed in blazars. This remains as one of the open questions in current astrophysics and has prompted the development of several theoretical models. The models either have leptons or hadrons as the primary accelerated particles in the jet, which then radiate directly or through the production of secondary particles, which in turn emit $\gamma$-rays.

\section{Leptonic Models}

In leptonic models, electrons and positrons are assumed to be the primary accelerated particles in the jet. The $\gamma$-rays are then produced by the scattering of low-energy photons to high energies via the Inverse Compton (IC) process described in section 1.1.1. However, there is no clear consensus about the origin of the low energy photons: in the Synchrotron Self-Compton (SSC) model [170, 29], the low-energy photons are produced by the relativistic electrons via synchrotron radiation in the magnetized jet. The synchrotron photons collide with the same population of relativistic electrons and are up-scattered to gamma-ray energies by inverse Compton. Their frequency $\nu_{I C}$ is related to the strength of the magnetic field $B$ and the electron energy $E_{e}$ (see eq.1.3),

$$
\nu_{I C} \propto \nu_{\text {syn }} \times E_{e}^{2} \approx\left(B \times E_{e}^{2}\right) \times E_{e}^{2} \approx B \times E_{e}^{4}
$$

It follows that the IC energy distribution springs from the synchrotron spectrum (shifted to higher energies). The observed turn-ons and roll-offs in the SED could be attributed to the low and high energy cutoffs in the electron spectrum.

The External Compton Scattering (ECS) models assume that the low-energy pho- 
tons are originated outside the jet. The possible sources of external radiation include: accretion disk photons radiated directly into the jet [57], accretion disk photons scattered by emission-line clouds or dust into the jet [244], or synchrotron radiation re-scattered back into the jet by broad-line-emission clouds [92]. If the low-energy photons interact with the jet with any angle but from behind (i.e. they do not come directly from the disk), then their energies and density are strongly Doppler boosted in the frame of the jet, thereby enhancing the IC process.

Synchrotron and external photons are known to be present in the jet environment and in consequence, both SSC and ECS processes are expected to occur. The open question remains, to what degree is one or the other (or none), responsible for the bulk of the $\gamma$-ray emission. Different classes of blazars (or blazars during different flaring states), could be dominated by different emission mechanisms. For example, BL Lacs are believed to have less matter in their central regions (given their weak or absent emission lines) and it is reasonable to expect a low contribution from ECS-type mechanisms.

\section{Hadronic Models}

In hadronic models, the accelerated particles responsible for the $\gamma$-ray emission are protons instead of leptons. Because of their large mass, protons are less susceptible to radiation losses and can be accelerated up to energies of $10^{20} \mathrm{eV}$. As explained in section 1.1.1.1, once the protons cross the threshold for photopion and pair production a multitude of secondary particles is produced. Electron-positron pairs lose their energy quickly via synchrotron radiation or inverse Compton, thus creating $\gamma$-rays. Gamma-rays interact among themselves or with external photons and pair produce, creating yet more electron-positron pairs. This chain reaction continues until the $\gamma$-ray energy is low enough to escape the source before being absorbed again.

Synchrotron proton models $[5,194]$ are another type of hadronic emission mech- 
anism that assumes that electrons and protons are co-accelerated in the same shock. In this model, the $\mathrm{TeV}$-photon emission of BL Lac sources is due to synchrotron radiation of relativistic protons in the highly magnetized emission region, while synchrotron radiation from co-accelerated electrons is responsible for the spectrum hump observed at lower energies. Both photopion production and proton synchrotron models could be at play: it has been argued that photopion production is the dominant emission mechanism in LBLs, while proton-synchrotron radiation is responsible for the TeV-photon emission of HBLs [194].

The generation of neutrinos as by-product of these processes is an important signature of hadronic models. The detection of neutrino fluxes from blazars in detectors like IceCube [146] would confirm protons as the primary accelerated particles.

\subsection{Dissertation Outline}

This chapter introduced the field of $\gamma$-ray astrophysics and gave a brief overview of blazar phenomena. Blazars have been observed with EGRET and ground-based instruments and are known to be the dominant class of extragalactic $\gamma$-ray sources. Although the advance in the understanding of blazars since their discovery has been spectacular, important questions related to the nature of blazars as a $\gamma$-ray source and as a population remain unanswered. GLAST, with its large field of view, improved sensitivity, and wide energy range is expected to produce fundamental breakthroughs in the study of $\gamma$-ray emitting AGN.

The research in this dissertation describes how to use the blazars that GLAST will observe as a tool to probe the cosmic background radiation known as EBL (Extragalactic Background Light, described in Chapter 2). The EBL is strongly connected to gamma-ray astrophysics because $\gamma$-rays emitted by blazars (or any other extragalactic source) are subject to absorption due to pair-production with EBL photons. 
One exciting consequence of this effect is that the magnitude of this absorption can then be used to measure -or at least constrain- the column density of background photons between the source and the observer [257]. This idea has been applied successfully to $\mathcal{O}(\gtrsim 100 \mathrm{GeV})$ observations of nearby $(z<0.2)$ blazars by ground-based instruments to constrain the infrared part of the EBL (section 2.6). Nevertheless, GLAST will allow for a completely new approach to EBL studies, namely, study of the systematic attenuation of large numbers of blazars as a function of redshift. This is possible thanks to GLAST's sensitivity and wide bandpass, which will allow the number of known blazars to increase from about one hundred to thousands, with redshifts up to $z \sim 3-5$. Furthermore, because $\gamma$-ray sources to be observed by GLAST are distributed over a wide range of redshifts, EBL studies with GLAST will not only probe the total level of the background radiation, but its evolution as well.

The dissertation is organized as follows: Chapter 2 presents an overview of the current knowledge of the EBL density, its current observational constraints, and its interaction with $\gamma$-rays. The GLAST LAT instrument and its performance are described in Chapter 3. The impact of ACD backsplash effect to the acceptance of the instrument at high energies (which is crucial to EBL studies) is studied in Chapter 3 with data obtained during a beam test of the LAT calibration unit. Based on a solid characterization of the instrument, two different methods to detect and measure the EBL attenuation of blazars are introduced in Chapter 5, and their results discussed. Conclusions are presented in Chapter 6 . 


\section{Chapter 2}

\section{Extragalactic Background Light}

\subsection{What is the EBL?}

Extragalactic Background Light (EBL) refers to the accumulation of energy release in the form of electromagnetic radiation since the decoupling of matter and radiation following the Big Bang [275]. By definition, EBL does not include foreground radiation from the Solar System, the Milky Way or other nearby galaxies, nor does it include the Cosmic Microwave Background radiation (CMB). Background in the form of high-energy radiation (X-rays and $\gamma$-rays) is not regarded a part of the EBL. This exclusion is justifiable because of the different origin of the high energy background: accretion-powered AGNs, as opposed to the EBL which is expected to be dominated by galaxy emission.

A schematic picture of the EBL spectrum is shown in Figure 2.1. The EBL consists of two spectral humps with different astrophysical origins. The blue hump located at UV-Optical-NIR ${ }^{1}$ wavelengths consists of the radiated output from stars (some other possible contributions will be discussed in the next section). The second hump (red line) corresponds to dust emission resulting from the absorption and re-emission

\footnotetext{
${ }^{1}$ NIR: Near-infrared part of the electromagnetic spectrum, illustrated in fig. 2.1. The NIR radiation is mostly due to redshifted Optical-UV emission from stars at high redshift $(z>3)$.
} 


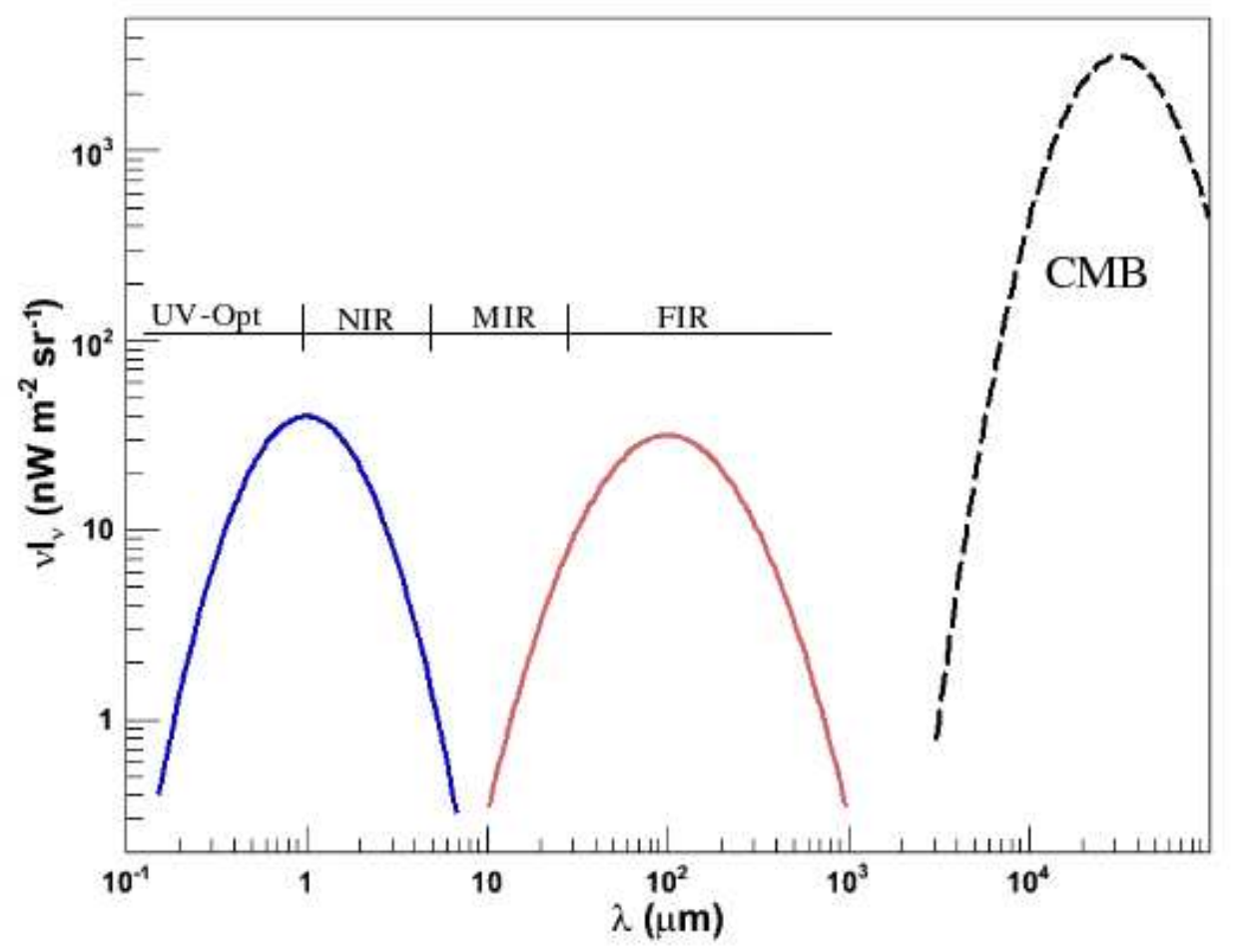

Figure 2.1: Schematic EBL spectrum as a function of wavelength. The EBL spectrum consists of two spectral humps: The blue hump at UV-Optical-NIR wavelengths is the radiated output from stars. The red hump at MIR (mid-infrared) and FIR (farinfrared) wavelengths results from the absorption and re-emission of starlight by the interstellar medium. The CMB spectrum (dashed black line) is presented here just for comparison purposes (since it is not considered part of the EBL). The location and size of the humps is just approximate; as will be described later, the precise shape and intensity of the EBL is not completely constrained from observations. The EBL spectrum is presented as a $\nu I_{\nu}$ plot, which is useful for showing the actual emitted power in each wavelength interval. 
of starlight by the interstellar medium ${ }^{2}$ (section 2.2.1.2). For comparison purposes, the plot also includes the CMB spectrum, even though, as explained before, it is not considered part of the EBL.

The EBL is by essence related to the formation and evolution of structure in the universe, which is one of the most important fields of research in astrophysics. The formation and evolution of cosmic structure results in the release of radiation, which ultimately constitutes the EBL. The radiation background is therefore a fossil of the structure formation process, and its measurement provides a fundamental insight into the history of the universe.

Something important to note is that a plot like fig. 2.1 only represents a snapshot of the EBL flux as observed at the present epoch $(z=0)$. The actual cosmic radiation background is not a static entity, it evolves continuously (as illustrated in fig. 2.2). First, light is redshifted by the cosmic expansion of the universe, resulting in an inexorable drift of radiated power from shorter to longer wavelengths. Second, the number and emission properties of the emitting sources change with time, as will be described in the next section.

\subsection{EBL Contributors}

\subsubsection{Conventional contributors}

The "conventional" contributors to the EBL are stars and the dust interacting with them. Stars are classified by their heavy-element abundance (nuclei with atomic number $Z>2$; also called metallicity), which correlates strongly with the star's age and with the type of galaxy where it can be found. Population I (hereafter Pop I) stars have high metallicity, tend to be hot, young, and luminous, and are usually

\footnotetext{
${ }^{2}$ The interstellar medium (ISM) is the term used to refer to the gas and dust that pervades the space between stars.
} 


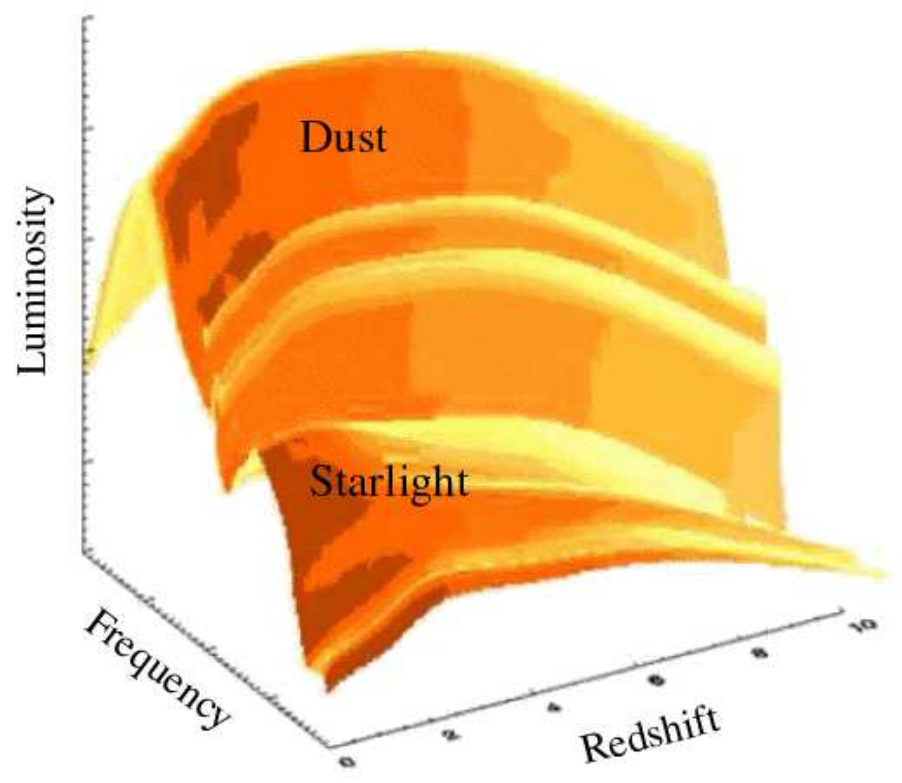

Figure 2.2: Adapted from [222]. Model-generated EBL density evolution as a function of redshift. This plot serves to illustrate the dynamic nature of the cosmic radiation fields.

found in the arms of spiral galaxies. Population II (Pop II) stars have low metallicity, are cooler, older and less luminous, and are usually found in globular clusters [221]. In a very simple picture of stars genealogy, Pop I stars form from the gas enriched by previous stars (Pop II) that went supernovae. In turn, Pop II stars are thought to be formed from (not yet observed) Population III stars (hereafter Pop III) [221]. Pop III stars have zero metallicity, and would have formed (and died) early in cosmic history. They will be discussed in section 2.2.2.

\subsubsection{Emission from Normal Stellar Populations}

The study of emission lines from quasars and the colors of distant galaxies [142] show that matter with non-zero metallicity was present early in the history of the universe. Therefore, all the stars responsible for the observed release of nuclear energy are classified as Pop I and II stars. Given the current theory of stellar formation and evolution, it is expected that star formation processes in this distant, but already 
metal-rich medium, led to to stellar populations similar to those observed today in the local universe.

In galaxies with ongoing star formation, the conversion of gas to stars takes place in bursts, or "starbursts". A group of stars produced during the same starburst episode will have a stellar mass distribution described by a universal Initial Mass Function (IMF) [234]. The lifetime and emission profile of a star are determined by its mass and original metallicity, therefore the IMF can be used to calculate the collective emission of a stellar population. Massive stars $\left(\mathrm{M}>10 \mathrm{M}_{\odot}\right)$ have short lives $\left(\sim 10^{7}\right.$ years) and produce the bulk of their output at UV wavelengths. Conversely, low-mass stars are long-lived, drift into the main sequence ${ }^{3}$, and radiate predominantly in the optical band. At any given instant, the combined spectral energy distribution (SED) of a stellar population depends on of the time elapsed since the burst that created it. Globally, the spectrum emitted by a typical galaxy consists thus of the superposition of SEDs from individual starbursts.

\section{UV Absorption in Stellar Populations}

The single most important correction to the galaxy-SEDs mentioned above is due to the absorption and re-emission of light by gas and dust inside the galaxy (ISM), or by material in the Intergalactic Medium (IGM). Atomic Hydrogen (H) is the most common absorber and its absorption properties follow from its well-known energy levels:

$$
E_{n}=-\frac{13.6 \mathrm{eV}}{n^{2}} \quad ; \quad n=1,2,3, \ldots
$$

A photon with energy over $13.6 \mathrm{eV}$ can break loose the single electron in a Hydrogen atom with any remainder energy going into the kinetic energy of the electron.

\footnotetext{
${ }^{3}$ Stars in the main sequence have attained stable hydrostatic equilibrium between radiation pressure and gravitational pull. The star will remain in such state until it has burned most of its Hydrogen into Helium)
} 
Conversely, if the energy of the photon is below $13.6 \mathrm{eV}$, the photon can still be absorbed if its energy is equal to the difference between any two energy levels $(\Delta E)$ in the Hydrogen atom

$$
\begin{aligned}
\Delta E=13.6\left(\frac{1}{n^{2}}-\frac{1}{m^{2}}\right) e V \quad n & =1,2,3, \ldots \\
m & =n+1, n+2, \ldots
\end{aligned}
$$

Of particular importance are the transitions known as Lyman $(n=1$ to $m$ transitions) and Balmer $(n=2$ to $m)$ series. They are the most common and often dominate the absorption spectrum ${ }^{4}$.

When a star is surrounded by neutral Hydrogen (i.e. in a HI cloud), all radiation above $13.6 \mathrm{eV}$ is subject to strong absorption (Lyman limit) with line absorption likely at discrete wavelengths (Lyman and Balmer series). The inverse process is also present, with emission resulting from the recombination of the free electrons with the Hydrogen ions. UV, visible and IR radiation result from this process as the electrons cascade down the Hydrogen energy levels.

Hot massive stars are particularly bright at UV wavelengths, and consequently, they ionize the gas clouds around them. Fully-ionized Hydrogen clouds are known as HII regions and consist of Hydrogen ions and free electrons in thermodynamic equilibrium. In addition to the radiation resulting from the recombination described above, when an electron passes near a proton but does not recombine, free-free scattering takes place, resulting in the emission of a continuum spectrum (bremsstrahlung radiation described in section 1.1.1.1). HII regions around hot massive stars are strong emitters of free-free radiation at IR and radio wavelengths.

\footnotetext{
${ }^{4}$ Transitions from $n=1$ to $m=2$ in the Lyman series are known as Ly $\alpha$, from $n=1$ to $m=3$ as $\operatorname{Ly} \beta$, etc. The same applies to the Balmer series.
} 


\subsubsection{Dust emission from galaxies}

Interstellar dust consists of grains (silicate and graphite, with size ranging from $10^{-9}$ to $10^{-5} \mathrm{~m}$ ), and macromolecules, commonly identified as polycyclic aromatic hydrocarbons [69]. The existence of large amounts of cosmic infrared radiation in the 10-1000 $\mu \mathrm{m}$ regime (section 2.3.2) reveals that dust is common in the universe, since only dust can efficiently absorb a significant fraction of the power radiated by stars and accretion sources, and re-emit it at infrared wavelengths [69]. Although the physical principle behind dust emission is simple, the detailed emission spectrum is rather complex because of the intrinsic peculiarities of the dust (microscopic properties, abundance, composition, and spatial distribution with respect to the UV-optical sources). Given the right conditions in terms of dust content and location, the reradiated emission from dust could be the dominant form of the total luminosity of a galaxy.

Ultraluminous Infrared Galaxies (ULIRGs) are such type of galaxy. ULIRGs were first discovered with the InfraRed Astronomical Satellite (IRAS) [246] with IR luminosities above $10^{12} L_{\odot}$. Most of these galaxies are dust obscured starburst galaxies or mergers, although some have been identified as AGN. Galaxy number counts of ULIRGs indicate that IR-luminous galaxies evolved more rapidly than their optical counterparts and could have made a bigger contribution (2 to 3 times larger than estimated from optical-UV alone) to star formation at intermediate redshifts $(z \lesssim 1.5)$ [76]. So, although ULIRGs are not predominant at the present epoch $(z=0)$, a significant fraction of the EBL at infrared wavelengths could be attributed to them. 


\subsubsection{Additional Contributors}

\subsubsection{Quasars / AGN}

As discussed in Chapter 1, AGN are broadband sources, and thus, contribute to the EBL. However, AGN are usually ignored in synthesis models of the EBL (see section 2.5) because of the current thinking that this contribution is small. This assumption is based on energy budget arguments [162]: the total observed mean mass density of quasar remnants (i.e. black holes) $\rho_{B H} \simeq(3 \pm 2) \times 10^{6} \mathrm{~h} \mathrm{M}_{\odot} \mathrm{Mpc}^{-3}$ could have contributed by accretion a maximum of $\frac{c}{4 \pi} \epsilon \rho_{B H} c^{2}\left\langle(1+z)^{-1}\right\rangle$ (where $\epsilon \sim 0.07$ is the efficiency to convert rest mass into radiated energy) to the EBL flux observed today. Thus, for a mean redshift distribution $\langle z\rangle \sim 2$, AGN are expected to produce less than $10-20 \%$ of the total EBL. The total AGN contribution to the EBL is also constrained by observational studies of the relation between AGN, hard x-ray and sub-millimeter sources [18] and by theoretical models for the infrared background contribution from obscured X-rays sources [99] (see [107, 135] for a review).

\subsubsection{Population III stars}

In the aftermath of the Big Bang, the matter content of the universe consisted of Hydrogen and Helium only, with no heavy elements present by the end of the radiation era. It is believed that in this "metal-free" environment the first stars formed, radiated and finally exploded in violent supernovae giving origin to the first metals ${ }^{5}$. This hypothetical stellar population is known as Population III (Pop III). Over time, the remnants of these Pop III stars had enough metallicity to form the Pop I and II stars observed today.

From simulations based on the theory of star formation, Pop III stars are expected

\footnotetext{
${ }^{5}$ Very massive Pop III stars $\left(\gtrsim 240 M_{\circ}\right)$ are not expected to explode as supernovae, but rather to collapse directly to a black hole, in which case they do not enrich the metallicity of the interstellar medium. An upper limit to the number of Pop3 stars and their radiative output based on metal abundance should take this into consideration.
} 


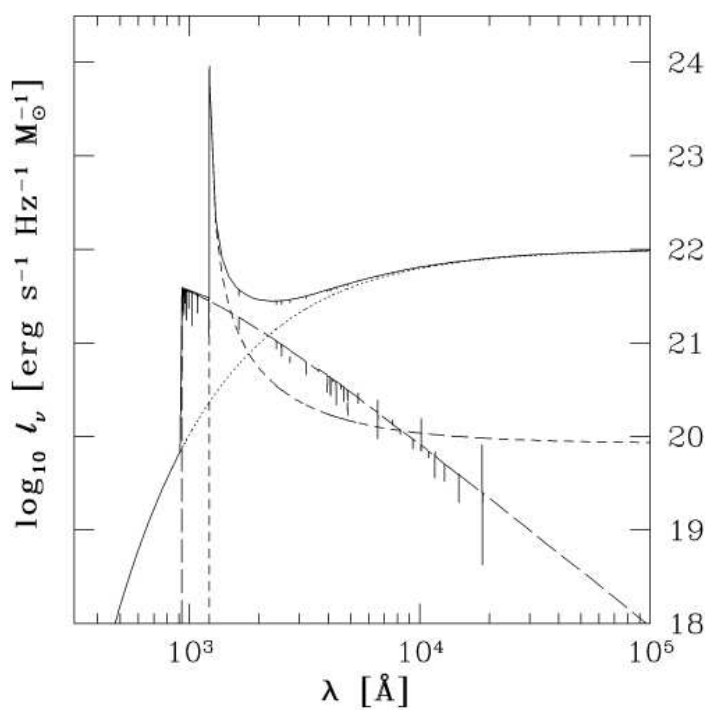

Figure 2.3: Spectrum of a Pop III star as a function of wavelength (in units of angstrom, $1 \AA=10^{-10} \mathrm{~m}$ ), from [236]. Three different components are present: the long-dashed line represents the emission from the star, completely absorbed shortward of the Lyman limit. The dotted line is the free-free (bremsstrahlung) emission from the nebula surrounding the star. The short-dashed line is the Ly $\alpha$ recombination emission corrected for scattering. All contributions are calculated in the rest frame of the star. This calculation in particular assumes that all the UV radiation over $13.6 \mathrm{eV}$ is completely absorbed by the halo around the star. Over time, the halo will become fully ionized and UV photons will escape.

to form differently from normal stellar populations. For a star-forming process in general, the gravitational collapse of the gas cloud leads to the fragmentation of the cloud into masses of the order $M \propto T^{3 / 2} \rho^{-1 / 2}[113]$ ( $T$ being the temperature and $\rho$ the density of the cloud). These masses continue to break into smaller pieces until the density is high enough to trap the thermal radiation resulting from the collapse. This leads to inefficient cooling and the increase in temperature and density eventually leads to nuclear burning (i.e. a star is born). In the specific case of Pop III stars, the current $\Lambda \mathrm{CDM}^{6}$ simulations suggest that the fragmentation is very inefficient for collapsing clouds at $z \sim 10-30$, and thus, Pop III stars could have been very massive $\left(M>100 M_{\odot}\right)[37]$.

\footnotetext{
${ }^{6} \Lambda \mathrm{CDM}$ stands for $\Lambda$ Cold Dark Matter cosmology, which is the accepted cosmological model following the results from WMAP [250]
} 
Once formed, Pop III stars are expected to radiate close to the Eddington limit $L \sim L_{E d d} \simeq 10^{38} \frac{M}{M_{\odot}} \operatorname{erg~s}^{-1}$ for most of their short lives $\left(t \sim 10^{6}\right.$ years $)$. Their spectrum can be described by black-body-type radiation of temperature $T \sim 10^{5} \mathrm{~K}$ [268], with UV absorption shortwards of the Lyman limit as discussed in the previous section. Figure 2.3 shows the calculated spectrum for a Pop III star [236].

Pop III stars remain a hypothetical concept and a direct detection is unlikely. Nevertheless, their net contribution to the EBL could be significant. If Pop III are indeed massive stars, each unit mass of Pop III star emits $\gtrsim 10^{5}$ more light than normal stars [135]. Detection of this "excess" contribution could be achieved with measurements of the fluctuations of the cosmic IR diffuse background. Recent observations with the Spitzer telescope indicate anisotropies of the IR background that are consistent with such signature [136].

Independent confirmation of the infrared excess due to Pop III objects could be obtained from the observation of a $\gamma$-ray optical depth that cannot be explained by the EBL contribution from galaxies alone [134]. If Pop III stars produced even a fraction of the claimed NIR excess, they would provide an abundant source of $\sim$ $(0.1-0.3)(1+z) \mathrm{eV}$ photons at high $z$, since its density would scale as $\propto(1+z)^{3}[134]$. However, as will become clear in section 1.5, the contribution from galaxies to the EBL at high redshifts is not well understood and thus very different levels of EBL absorption are possible. Nevertheless, GLAST observations could provide important constraints on the emission from Pop III objects.

\subsubsection{Exotic Sources}

Non-nuclear, non-AGN possible contributions to the EBL include radiation from brown dwarfs and the decay of primordial particles. Brown dwarfs are bodies whose mass is below the limit required for Hydrogen burning $\left(\sim 0.08 \mathrm{M}_{\odot}\right)$. Assuming that 
these objects represented all the dark matter required to close the universe (which is not the case), they would contribute $3 \mathrm{nW} \mathrm{m}^{-2} \mathrm{sr}^{-1}$ at most in the $10-100 \mu \mathrm{m}$ region [39], still a small amount if compared with the contribution from normal galaxies $\left(\sim 40 \mathrm{nW} \mathrm{m}^{-2} \mathrm{sr}^{-1}\right)$.

If real, the radiated output from the decay or interaction of primordial particles would depend on their number density, particle mass and on redshift distribution. While a wide range of intensities can be predicted [32, 184], no physical evidence favors one set of assumptions over the others, and the existence itself of such primordial particles remains highly conjectural.

In summary, it is justifiable to expect that the energy content of the EBL results mostly from nuclear processes inside stars. Section 2.3 reviews the current limits and direct detections of the EBL flux, while section 2.5 deals with EBL models in which the background density over cosmic time is predicted from astrophysical principles.

\subsection{Direct Measurements}

Direct measurements of the EBL intensity are very difficult. The EBL has no spectral signature, since its spectrum depends in a nontrivial way on the characteristics of the sources, on their cosmic history, and on the process of dust formation around these sources. More important, the EBL flux is excessively weak compared to the foreground from other astrophysical sources ${ }^{7}$. Foreground sources include stars in the galaxy, diffuse emission from the interplanetary dust (IPD) and the ISM. At submillimeter wavelengths the CMB becomes dominant and also has to be subtracted from the EBL flux.

\footnotetext{
${ }^{7}$ Emission by the atmosphere is also a source of foreground emission for ground-based observations.
} 


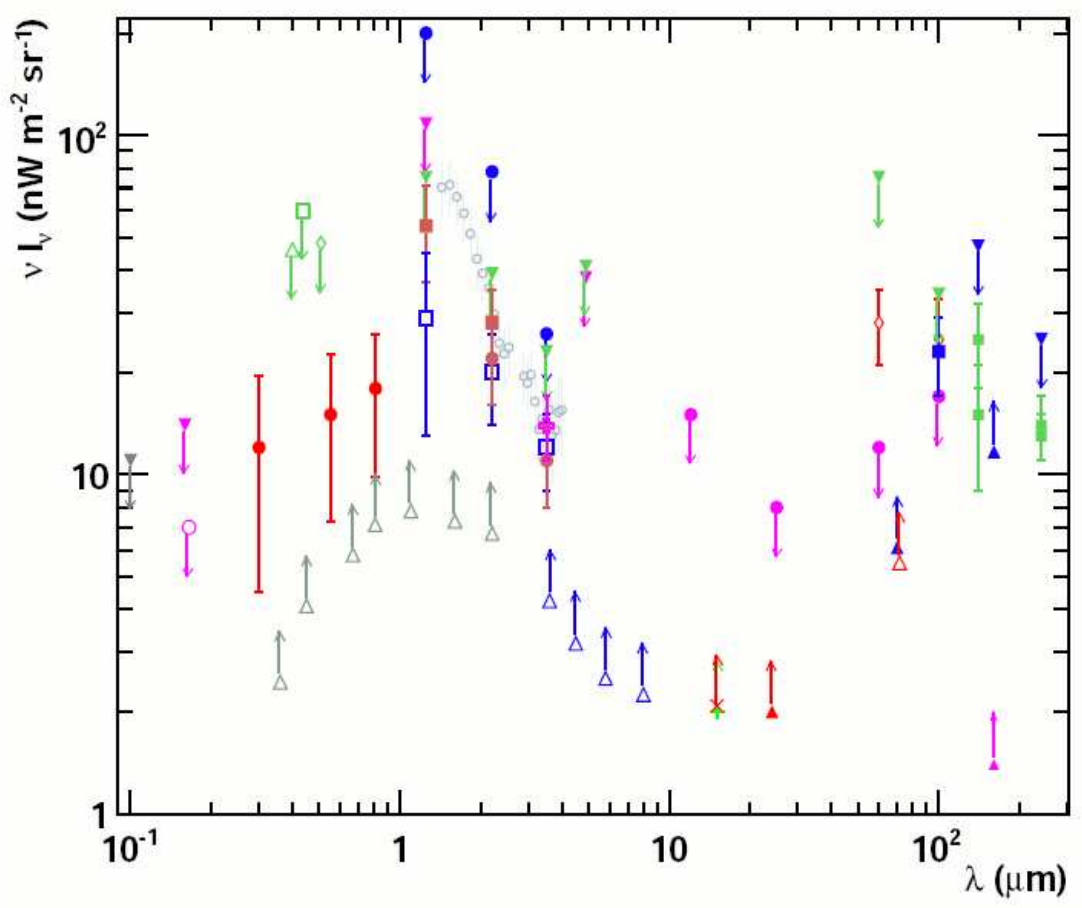

Figure 2.4: EBL measurements and limits, from [183]. Upper limits in the UV to optical: Edelstein et al. (2000) (gray filled triangle, [73]), Martin et al. (1991) (open pink circle, [172]), Brown et al. (2000) (filled pink triangle, [36]), Mattila (1990) (open green triangle, [176]), Toller (1983) / Leinert et al. (1998) (open green square, [156, 266]), Dube et al. (1979) / Leinert et al. (1998) (open green diamond, [65, 156]); Tentative detection in the UV/optical: Bernstein et al. (2002, 2005) (filled red circle, [20]); Lower limits from source counts: Madau \& Pozzetti (2000) (open gray triangles, [162]), Fazio et al. (2004) (open blue triangles, [79]), Elbaz et al. (2002) (green cross, [75]), Metcalfe et al. (2003) (red x, [186]), Papovich et al. (2004) (filled red triangle, [214]), Dole et al. (2006) (filled pink triangles, [62]), Frayer et al. (2006) (open red triangle, [87]); Detections in the near IR: Dwek \& Arendt (1998) (open pink cross, [71]), Gorjian et al. (2000) (filled brown circle, [97]), Wright \& Reese (2000) (open blue squares, [280]), Cambresy et al. (2001) (filled brown squares, [40]), Matsumoto et al. (2005) (small open gray circles, [178]); Upper limits from direct measurements: Hauser et al. (1998) (filled green triangles, [108]), Dwek \& Arendt (1998) (filled pink triangles, [71]), Lagache \& Puget (2000) (filled blue triangles, [151]); Upper limits from fluctuation analysis: Kashlinsky et al. (1996) (filled blue circles, [138]), Kashlinsky \& Odenwald (2000) (filled pink circles, [137]); Lower limits from stacking analysis in the far-IR: Dole et al. (2006) (blue triangles, [62]); Detections in the farIR: Hauser et al. (1998) (filled green squares, [108]), Lagache \& Puget (2000) (filled blue square, [151]), Finkbeiner et al. (2000) (open red diamonds, [83]). 
The zodiacal light from the $\mathrm{IPD}^{8}$ is the brightest foreground at most IR wavelengths $(1.25-140 \mu \mathrm{m})$, with other substantial contributions arising from starlight at NIR wavelengths $(1.25$ to $3.5 \mu \mathrm{m})$, and from ISM emission for wavelengths greater than $60 \mu m$.

The observations described below attempt to measure the EBL flux through different techniques and analysis. A candidate detection should result in a positive residual signal that is in excess of the random and systematic uncertainties from the measurements and the foreground determination. In addition, the residual signal must be isotropic and seemingly independent from radiation originated in the solar system or the galaxy [107]. The collective limits and measurements of the EBL obtained from direct astronomical observations are summarized in fig. 2.4.

\subsubsection{UV-Optical}

At UV-Optical wavelengths two different techniques have been used to measure the EBL intensity: light integration of extragalactic source counts and foreground subtraction of the sky brightness.

\section{Light Integration of Extragalactic Source Counts}

In this technique, the light from galaxies is integrated down to the faintest fluxes possible with the currently available instruments. A necessary condition for a meaningful reported integrated flux is evidence that the sum has started to converge. Technically, convergence is assumed when the logarithmic slope of the differential galaxy count per magnitude interval $(d \log N / d m)$ drops below a value of 0.4 at faint magnitudes [107].

\footnotetext{
${ }^{8}$ The zodiacal light at $\sim 1-3.5 \mu \mathrm{m}$ is produced by sunlight reflecting off dust particles in the solar system and known as the interplanetary dust (IPD) cloud. At longer wavelengths the zodiacal light is originated by thermal emission from the IPD instead. This cloud of dust is located in a lens-shaped volume of space centered on the Sun and extending well out beyond the orbit of Earth.
} 


\begin{tabular}{ccc}
\hline$\lambda(\mu m)$ & $\nu I_{\nu}\left(\mathrm{nW} \mathrm{m}^{-2} \mathrm{sr}^{-1}\right)$ & Reference \\
\hline \hline 0.1595 & $>2.9_{-0.4}^{+0.6}$ & {$[90]$} \\
0.2365 & $3.6_{-0.5}^{+0.7}$ & {$[90]$} \\
0.36 & $2.9_{-0.4}^{+0.6}$ & {$[162]$} \\
0.45 & $4.6_{-0.5}^{+0.7}$ & {$[162]$} \\
0.67 & $6.7_{-0.9}^{+1.3}$ & {$[162]$} \\
0.81 & $8.0_{-0.9}^{+1.6}$ & {$[162]$} \\
1.1 & $9.7_{-1.9}^{+3.0}$ & {$[162]$} \\
1.6 & $9.0_{-1.7}^{+2.6}$ & {$[162]$} \\
2.2 & $7.9_{-1.2}^{+2.0}$ & {$[162]$} \\
\hline
\end{tabular}

Table 2.1: Integrated Galaxy Light measurements. Adapted from [107]. All measurements presented here should be considered lower limits to the EBL.

It is important to note that this cumulative brightness is just a strict lower limit to the EBL. It is possible that a significant fraction of the UV-optical flux is originated by low-surface-brightness galaxies that cannot be resolved and therefore would be missed from source counts. Also, the possibility of a truly diffuse contribution to the background cannot be discarded from source count observations. Truly diffuse emission can only be detected by instruments with absolute surface photometry capabilities.

Madau and Pozetti [162] used the Hubble Space Telescope (HST) northern and southern deep fields in combination with other ground observations to add the light from galaxies at different energy bands down to very faint magnitudes. Gardner et al [90] extended the work to shorter UV wavelengths using observations made with the Space Telescope Imaging Spectrograph (STIS) on board HST combined with galaxy counts obtained with balloon-borne experiments. Their results are presented in Table 2.1 . 


\begin{tabular}{cccc}
\hline$\lambda(\mu m)$ & $\nu I_{\nu}\left(\mathrm{nW} \mathrm{m}^{-2} \mathrm{sr}^{-1}\right)$ & Instrument/Comment & Reference \\
\hline \hline 0.10 & $<11$ & Voyager UVS & {$[73,198]$} \\
0.1595 & $<14(10 \pm 2)$ & HST/STIS & {$[36]$} \\
0.165 & $<7.0(5.6 \pm 0.7)$ & Shuttle UVX & {$[172]$} \\
0.3 & $12 \pm 7$ & HST $/$ LCO & {$[20]$} \\
0.3 & $22.2-27.3$ & Correction to $[20]$ & {$[175]$} \\
0.4 & $<46(26 \pm 10)$ & "Dark Cloud" Method & {$[176]$} \\
0.44 & $<60(10 \pm 25)$ & Pioneer 10 & {$[266,156]$} \\
0.5115 & $<48(30 \pm 9)$ & Ground-based photometer & {$[65,156]$} \\
0.550 & $17 \pm 7$ & HST /LC & {$[20]$} \\
0.550 & $84.7-113.9$ & Correction to $[20]$ & {$[175]$} \\
0.814 & $24 \pm 7$ & HST /LCO & {$[20]$} \\
0.814 & $84.8-114.2$ & Correction to $[20]$ & {$[175]$} \\
\hline
\end{tabular}

Table 2.2: Adapted from [107]. Errors are $1 \sigma$. Upper limits are $2 \sigma$. Values in parentheses are measurements and their uncertainty. UVS is the Ultraviolet Spectrometer on board the Voyager mission, STIS is the Space Telescope Imaging Spectrograph on the Hubble Space Telescope (HST) and LCO stands for Las Campanas Observatory.

\section{Foreground Subtraction of the Sky Brightness}

The second technique consists of measuring the total sky brightness and then removing the contribution from known foreground sources, namely, the diffuse galactic light and the zodiacal light. Although the principle is quite simple, determining the value of the foregrounds is very challenging from a technical point of view. Pioneering work in foreground subtraction combined with early observations from space resulted in the upper limits that are listed in Table 2.2 (see [107] for a thorough compilation).

Bernstein et al [20] reported the first detection of EBL flux at wavelengths of 3000, 5500 and $8000\left(1 \AA=10^{-10} \mathrm{~m}\right)$, by measuring the absolute sky brightness with the Hubble space telescope. By using a ground-telescope at Las Campanas Observatory to obtain simultaneous spectrophotometry of the sky in the HST field of view, they were able to measure and then subtract the foreground zodiacal light from the HST observations. The reported values are presented in Table 2.2. It has been argued that 
these results are flawed because of errors in the determination of the zodiacal light from their ground-based observations [175]. The applied corrections led to higher nominal fluxes at the same time that the $1 \sigma$ errors became so large that the reported detection has been put into question. In conclusion, direct measurement of the UVoptical part of the EBL remains a difficult problem.

It should be noted also that the limits and direct measurements of the optical-UV discussed here are relevant to the EBL flux as observed in the current epoch $(z=0)$, and do not trace the EBL history. In this regard, GLAST observations of the EBL attenuation of $\gamma$-ray blazars offer a unique insight into the evolution of the EBL over cosmic history.

\subsubsection{IR}

Measurement of the EBL flux at infrared wavelengths has been attempted through different analyses aimed to extract the isotropic, mean level signal of the Cosmic Infrared Background (CIB) from ground- and space-based instruments. Although several detections have been reported, it is fair to say that they are not regarded as robust (especially in the NIR). This is due to the systematic uncertainties arising from the modeling and subtraction of zodiacal light and starlight, which ultimately dominate the analysis. An alternate methodology was proposed by Kashlinsky et al [138] via the measurement of the CIB anisotropy from its angular power spectrum. In this approach, the fluctuations in the intensity of the CIB form a distinct spectral and spatial signal that can be used to set limits on the CIB. A brief summary is presented below, organized according to the instrument used to collect the data. A complete review of the relevant literature can be found in [107, 135]. 


\subsubsection{COBE-DIRBE}

The Diffuse InfraRed Background Experiment (DIRBE) [31] on board the Cosmic Background Explorer (COBE) was designed to measure, or at least put strict limits on, the CIB. DIRBE consisted of a 10-band photometer system that covered the $1.25-240 \mu m$ range with an angular resolution of $0.7^{\circ}$. The 41 -week maps from DIRBE observations were used to model the contributions from the Interplanetary Dust (IPD) [141] and the Galaxy[10]. These contributions were then removed from the total flux and the residual was considered as candidate CIB flux. The criteria for detection by the DIRBE team included a $3 \sigma$ excess and isotropy in the residual signal. A firm detection was reported at 140 and $240 \mu \mathrm{m}$ and only upper limits at shorter wavelengths[108]. This pioneering work was followed by other analysis aimed to achieve a better determination of the foreground emission (table 2.4).

As mentioned before, an alternate technique consists of probing the CIB spectral and spatial structure instead of its mean level. The technique was applied to the DIRBE sky maps (with zodiacal light subtracted) and a residual fluctuation was identified with the CIB [137]. The reported values from the different analyses are listed in table 2.3 .

\subsubsection{COBE-FIRAS}

The Far Infrared Absolute Spectrometer (FIRAS) [31] on board COBE was designed to measure the spectrum of the CMB and the FIR background. FIRAS covered the wavelength range $104-5000 \mu \mathrm{m}$ with an angular resolution of $7^{\circ}$. CIB measurement from the FIRAS data [85, 152, 225] required subtraction of the CMB (in the region where it overlaps) and accounting for the IPD contribution (which was obtained by extrapolation of the model obtained from DIRBE [141]). Different analyses (subject to different systematic uncertainties) yielded a consistent residual isotropic background, 


\begin{tabular}{ccc}
\hline$\lambda(\mu m)$ & $\delta\left(\nu I_{\nu}\right)$ & $\nu I_{\nu}$ \\
\hline \hline 1.25 & $15.5_{-7.0}^{+3.7}$ & $<200$ \\
2.2 & $5.9_{-3.7}^{+1.6}$ & $<78$ \\
3.5 & $2.4_{-0.9}^{+0.5}$ & $<26$ \\
4.9 & $2.0_{-0.5}^{+0.25}$ & $<13$ \\
12 & $\lesssim 1$ & $<15$ \\
25 & $\lesssim 0.5$ & $<8$ \\
60 & $\lesssim 0.7$ & $<12$ \\
100 & $\lesssim 1$ & $<17$ \\
\hline
\end{tabular}

Table 2.3: Measurements of infrared background fluctuations from [137, 138], compiled by $[107,135] .\left[\delta\left(\nu I_{\nu}\right)\right]^{2}$ is the variance of $\nu I_{\nu}$ in units of $\left(\mathrm{nW} \mathrm{m}^{-2} \mathrm{sr}^{-1}\right) . \nu I_{\nu}$ in units of $\left(\mathrm{nW} \mathrm{m}^{-2} \mathrm{sr}^{-1}\right)$ is the reported limit on the CIB inferred from the fluctuation measurement.

whose mean value is a function of the frequency $\nu$ and is well-described by

$$
a\left(\frac{\nu}{\nu_{0}}\right)^{k} \nu B(\nu, T)
$$

with $a=8.8 \times 10^{-5}, k=1.4, T=13.6 \mathrm{~K}, \nu_{0}=3 \times 10^{12} \mathrm{~Hz}$ and where $B(\nu, T)$ is the Planck function,

$$
B_{v}(T)=\frac{2 h^{2}}{c^{2}} \frac{\nu^{3}}{e^{h \nu /(K T)}-1}
$$

\subsubsection{IRTS-NIRS}

The Near Infrared Spectrometer (NIRS) [206] on board the Infrared Telescope in Space (IRTS) was designed specifically to measure the spectrum of the CIB. It covered the wavelength range from 1.4 to $4 \mu \mathrm{m}$ in 24-independent bands with a spectral resolution of $0.13 \mu \mathrm{m}$. The analysis of 5 days of data resulted in a positive signal for the CIB flux after foreground subtractions [179, 178]. While the reported intensities 


\begin{tabular}{ccc}
\hline$\lambda(\mu m)$ & $\nu I_{\nu}\left(\mathrm{nW} \mathrm{m}^{-2} \mathrm{sr}^{-1}\right)$ & Reference \\
\hline \hline 1.25 & $<75$ & {$[108]$} \\
1.25 & $54.0 \pm 16.8$ & {$[40]$} \\
2.2 & $<39$ & {$[108]$} \\
2.2 & $22.4 \pm 6$ & {$[97]$} \\
2.2 & $23.1 \pm 5.9$ & {$[280]$} \\
2.2 & $20.2 \pm 6.3$ & {$[279]$} \\
2.2 & $27.8 \pm 6.7$ & {$[40]$} \\
3.5 & $<23$ & {$[108]$} \\
3.5 & $11.0 \pm 0.3$ & {$[97]$} \\
3.5 & $12.4 \pm 3.2$ & {$[280]$} \\
4.9 & $<41$ & {$[108]$} \\
12 & $<468$ & {$[108]$} \\
25 & $<504$ & {$[108]$} \\
60 & $<75$ & {$[108]$} \\
60 & $28.1 \pm 1.8($ stat $) \pm 7$ (sys) & {$[83]$} \\
100 & $<34$ & {$[108]$} \\
100 & $24.6 \pm 2.5$ (stat) \pm 8 (sys) & {$[83]$} \\
140 & $32 \pm 13$ & {$[239]$} \\
140 & $25.0 \pm 6.9$ & {$[108]$} \\
240 & $17 \pm 4$ & {$[239]$} \\
240 & $13.6 \pm 2.5$ & {$[108]$} \\
\hline
\end{tabular}

Table 2.4: Summary of DIRBE measurements as compiled by [107, 135]. 
around 2.2 and $3.5 \mu \mathrm{m}$ are consistent with previously reported upper limits, at shorter wavelengths the measurements from IRTS-NIRS are above the upper limits listed in Table 2.4. This near-infrared background excess (NIRBE) has been interpreted by some as the redshifted flux from the first stars [235], but (as will be discussed in section 2.6.4.1) recent $\mathrm{TeV}$ observations of the $\gamma$-ray spectrum of two blazars at $z \sim 0.18$ suggest a low NIR background flux, inconsistent with the bright flux discussed here. Dwek et al [67] argue against an extragalactic interpretation of the total flux reported from the IRTS-NIRS observations, and explain this excess as local foreground that has not been properly subtracted.

\subsubsection{NICMOS}

Observations of the Hubble Deep Field-North (HDFN) and the Hubble Ultra Deep Field (HUDF) with the Near Infrared Camera and Multi-Object Spectrometer (NICMOS) [16] on board the Hubble Space Telescope have been used by Thompson et al [263] to measure the intensity of the NIR background at $1.6 \mu \mathrm{m}$. Thompson et al find that the flux from resolved galaxies $\left(6.9_{-0.3}^{+3} \mathrm{nW} \mathrm{m}^{-2} \mathrm{sr}^{-1}\right)$ and from zodiacal light (455.0 $\mathrm{nW} \mathrm{m} \mathrm{m}^{-2} \mathrm{sr}^{-1}$ ) can account for the totality of the NIR flux, thus contradicting the existence of the NIR background excess reported by NIRS. Taken at face value, this result indicates that the EBL intensity at $1.6 \mu \mathrm{m}$ has already been measured and is equal to $6.9_{-0.3}^{+3} \mathrm{nW} \mathrm{m} \mathrm{m}^{-2} \mathrm{sr}^{-1}$.

\subsubsection{IRAS, ISO, SCUBA, SPITZER}

The observations described below were obtained from infrared telescopes designed to detect discrete sources, not to measure the infrared background. Nevertheless, these instruments were used intensively in sky-surveys, and their data was used to place lower limits on the CIB flux from the integrated light from source counts. Table 2.5 lists the reported values with the corresponding references. 
- The Infrared Astronomical Satellite (IRAS) was the first instrument to perform all-sky surveys at infrared wavelengths. The integrated light at 12, 25, 60 and $100 \mu \mathrm{m}$ is presented in table 2.5 .

- Observations with the infrared camera ISOCAM and infrared photometer ISOPHOT on board the Infrared Space Observatory (ISO) were used to find the integrated intensity at $7,12,15,90,150,170,175$ and $180 \mu \mathrm{m}$.

- The Sub-millimeter Common User Array (SCUBA) on the James Clerk Maxwell telescope obtained deep source counts at $850 \mu \mathrm{m}$. The integrated light at this wavelength accounts for most of the CIB flux detected with FIRAS.

- The Infrared Array Camera (IRAC) on board the Spitzer telescope found the galaxy contributions at $3.6,4.5,5.8$ and $8 \mu \mathrm{m}$. While at 3.6 and $4.5 \mu \mathrm{m}$ the integrated light seems to converge, at 5.8 and $8 \mu \mathrm{m}$ the saturation is not clear. In any case, the measured fluxes should be considered as lower limits to the CIB.

- The Multi-band Imaging Photometer System (MIPS) on board the Spitzer telescope was used to measure the contribution from galaxies at 24, 70 and $170 \mu \mathrm{m}$.

\subsection{EBL Density as a Cosmological and Astrophysi- cal Probe}

\subsubsection{Total EBL energy}

The integrated EBL intensity constrains the total energy budget of the physical processes involved in the emission (section 2.2). From the measurements discussed in section 2.3, upper and lower limits for the deposited energy in different wavelength intervals were obtained by Hauser and Dwek [107] and are presented in table 2.6. 


\begin{tabular}{cccc}
\hline$\lambda(\mu m)$ & $\nu I_{\nu}\left(\mathrm{nW} \mathrm{m}^{-2} \mathrm{sr}^{-1}\right)$ & Instrument & Reference \\
\hline \hline 3.6 & $5.27 \pm 1.02$ & SPITZER/IRAC & {$[79]$} \\
4.5 & $3.95 \pm 0.77$ & SPITZER/IRAC & {$[79]$} \\
5.8 & $\gtrsim 2.73 \pm 0.22$ & SPITZER/IRAC & {$[79]$} \\
7 & $1.7 \pm 0.5$ & ISO/ISOCAM & {$[8]$} \\
8 & $\gtrsim 2.46 \pm 0.21$ & SPITZER/IRAC & {$[79]$} \\
12 & $0.50 \pm 0.15$ & ISO/ISOCAM & {$[47]$} \\
15 & $2.7 \pm 0.6$ & ISO/ISOCAM & {$[75,186]$} \\
24 & $2.7_{-0.7}^{+1.1}$ & SPITZER/MIPS & {$[214]$} \\
25 & 0.02 & IRAS & {$[103]$} \\
60 & 0.4 & IRAS & {$[103]$} \\
70 & $7.1 \pm 1.0$ & SPITZER/MIPS & {$[62]$} \\
71.4 & $7.4 \pm 1.9$ & SPITZER/GOODS & {$[87]$} \\
90 & 1.0 & ISO/ISOPHOT & {$[131,177]$} \\
95 & 0.5 & ISO/ISOPHOT & {$[140]$} \\
100 & 0.2 & IRAS & {$[103]$} \\
150 & $\sim 1.0$ & ISO/ISOPHOT & {$[131]$} \\
160 & $13.4 \pm 1.7$ & SPITZER/MIPS & {$[62]$} \\
175 & 1.75 & ISO/ISOPHOT & {$[224]$} \\
180 & $\sim 1.2$ & ISO/ISOPHOT & {$[131]$} \\
450 & $2.4 \pm 0.7$ & SCUBA \& SPITZER & {$[243]$} \\
850 & $0.5 \pm 0.2$ & SCUBA & {$[24]$} \\
\hline
\end{tabular}

Table 2.5: Integrated galaxy light as compiled by [107, 135]. These measurements should be regarded as lower limits of the EBL flux at IR wavelengths.

\begin{tabular}{|c|c|c|}
\hline Intensity & $\begin{array}{c}\text { Range of values of the } \\
\text { integral }\left(\mathrm{nW} \mathrm{m}^{-2} \mathrm{sr}^{-1}\right)\end{array}$ & Wavelength Interval $(\mu \mathrm{m})$ \\
\hline \hline$I_{\text {stellar }}$ & $(19-100)$ & $0.16-3.5$ \\
\hline$I_{\text {Dust-MIR }}$ & $(11-58)$ & $3.5-140$ \\
\hline$I_{\text {Dust-FIR }}$ & $(13-17)$ & $140-1000$ \\
\hline$I_{\text {EBL }}($ total $)$ & $(43-175)$ & $0.16-1000$ \\
\hline
\end{tabular}

Table 2.6: Integrated EBL energy at different wavelengths from [107]. 


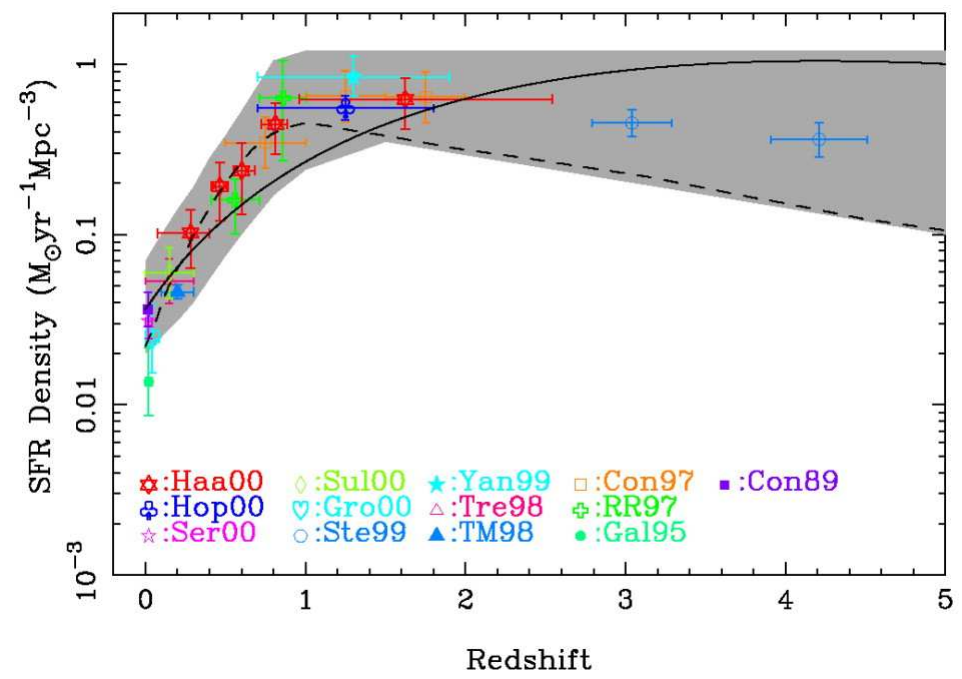

Figure 2.5: Comoving SFR density $\left(\dot{\rho}_{*}\right)$ as a function of redshift from [114]. The data points and SFR models (solid and dashed lines) have been compiled from references within [114]. The shaded area indicates the level of uncertainty.

The EBL energy density can be expressed as a fraction of the critical energy density $\left(\rho_{c} c^{2}\right)$ through the dimensionless quantity $\Omega_{\mathrm{EBL}}[107]$,

$$
\begin{aligned}
\Omega_{\mathrm{EBL}} & =\frac{4 \pi}{c} \frac{I_{\mathrm{EBL}}}{\rho_{c} c^{2}} \\
& \sim 10^{-6}-10^{-5}
\end{aligned}
$$

Thus, the roughly measured EBL intensity indicates that the total energy contained in the EBL is small when compared to other energy budgets in the universe. In particular, $\Omega_{\mathrm{EBL}} / \Omega_{\mathrm{CMB}} \sim 0.1$.

\subsubsection{Star Formation Rate (SFR)}

The average conversion rate of gas into stars is known as the star formation rate (SFR) and it is fundamentally related to the formation of structure in the universe and therefore, to the evolution of the EBL. Galaxy surveys and spectroscopic studies have revealed a consistent picture of the $\operatorname{SFR}([114,142,165]$ and references therein): from $z=0$ to $z=1$, it is generally agreed that the comoving space density of the SFR 
in galaxies $\left(\dot{\rho}_{*}\right)$, rises by an order of magnitude, and stays flat between $1<z<2$. The behavior of this evolution at higher redshifts, however, is not well understood. It is still unclear whether $\dot{\rho}_{*}$ peaks around $z \sim 1.5$ and decreases significantly thereafter, or if it stays flat to much higher redshifts (see fig. 2.5).

If the assumption is made that most of the EBL energy is produced by stars, then the EBL comoving luminosity density $\mathcal{L}$ can be directly related to the cosmic star formation rate ([107] and references therein):

The EBL intensity $I$ is the integral of $\mathcal{L}(z)$ over redshift:

$$
I_{E B L}=\left(\frac{1}{4 \pi}\right) \int \mathcal{L}(z)\left|\frac{d t}{d z}\right| \frac{d z}{1+z}
$$

where $d t / d z$ is given by

$$
\left|\frac{d t}{d z}\right|=\left(\frac{c}{H_{0}}\right) \frac{1}{1+z}\left[(1+z)^{2}\left(1+\Omega_{M} z\right)-z(2+z) \Omega_{\Lambda}\right]^{-1 / 2}
$$

where $H_{0} \simeq 71 \mathrm{~km} \mathrm{~s}^{-1} \mathrm{Mpc}^{-1}$ is the present day Hubble expansion rate, $\Omega_{M}=\rho_{M} / \rho_{c}$ is the present mass density of the universe normalized to the critical density, and $\Omega_{\Lambda}=\Lambda / 3 H_{0}^{2}$ is the dimensionless cosmological constant. The relation between $\mathcal{L}$ and $\dot{\rho}_{*}$ is given by the convolution [107]

$$
\mathcal{L}(t)=\int_{0}^{t} \rho_{*}(\tau) L_{b}(t-\tau) d \tau
$$

where $L_{b}(t)$ is the bolometric luminosity per unit mass of a stellar population of age $t$, which depends on the stellar IMF. Combining equations 2.6 and 2.8, and assuming a constant SFR $\left\langle\dot{\rho_{*}}\right\rangle$ and a Salpeter IMF $\left(\propto M^{-2.35} ; 0.1 M_{\odot}<M<120 M_{\odot}\right)$, the 
average SFR is related directly to the EBL intensity $I_{E B L}{ }^{9}[107]$ :

$$
\left\langle\dot{\rho}_{*}\right\rangle\left(\mathrm{M}_{\odot} \mathrm{yr}^{-1} \mathrm{Mpc}^{-3}\right)=(0.17-0.35) \times 10^{-2} I_{E B L}\left(\mathrm{nW} \mathrm{m}^{-2} \mathrm{sr}^{-1}\right)
$$

The roughly measured EBL intensity $I_{E B L}=43-175 \mathrm{nW} \mathrm{m}^{-2} \mathrm{sr}^{-1}$, leads to $\left\langle\dot{\rho}_{*}\right\rangle \sim 0.2 \mathrm{M}_{\odot} \mathrm{yr}^{-1} \mathrm{Mpc}^{-3}$, which is an order of magnitude higher than the measured value in the present epoch $\left(\sim 0.01 \mathrm{M}_{\odot} \mathrm{yr}^{-1} \mathrm{Mpc}^{-3}\right.$, from [163]), a clear indication that the SFR was higher in the past. In a more detailed analysis, the cosmic SFR evolves with redshift, and the measured EBL intensity is used to test the validity of a proposed star formation history. Unfortunately, with the current uncertainties in the EBL intensity, even very different star formation histories are found to be consistent with observations [70].

\subsubsection{Element Production}

Stars shine due to the fusion of Hydrogen into heavier elements, which is an exothermic reaction that liberates $0.7 \%$ of the rest mass energy. Following the literature convention, let $\mathrm{X}$ be the fraction of baryons in the form of Hydrogen and $\Delta X$ the fraction of $\mathrm{X}$ that is transformed into heavier elements. If the assumption is again made that most of the EBL is produced by stars, then the EBL comoving luminosity density is just the result of the nuclear fusion of Hydrogen and is given by ([107] and references therein)

$$
\mathcal{L}(z)=0.007 \dot{\rho}_{\Delta X}(z) c^{2}
$$

If the conversion rate of Hydrogen to heavier elements is assumed to be constant

\footnotetext{
${ }^{9}$ In [107] Hauser and Dwek use $\Omega_{M}=1$ and $\Omega_{\Lambda}=0$. Their result in eq. 2.9 has been recalculated using the now standard $\Lambda$ CDM cosmology values $\Omega_{M}=0.3$ and $\Omega_{\Lambda}=0.7$.
} 
over time ${ }^{10}$, then the integral in eq. 2.6 is straightforward and

$$
I_{\mathrm{EBL}}=\left(\frac{c}{4 \pi}\right) \times 0.007 \rho_{\Delta \mathrm{X}} c^{2}
$$

where $\rho_{\Delta \mathrm{X}}$ is the mass density of consumed Hydrogen over cosmic history, which can be expressed as a fraction of the baryons in the universe $\Delta X=\rho_{\Delta X} / \rho_{b}=\left(\Omega_{\Delta X} / \Omega_{b}\right)$, where $\rho_{b}$ is the baryonic mass density and $\Omega_{b}=(1.92 \pm 0.18) \times 10^{-2} h^{-2}$ is the fraction of the critical mass density constituted by baryons [208]. Therefore, the fraction of baryons used as nuclear fuel in stars can be expressed in terms of the EBL intensity [107]:

$$
\Delta X \simeq 2 \times 10^{-4} \times I_{\mathrm{EBL}}\left(\mathrm{nW} \mathrm{m}^{-2} \mathrm{sr}^{-1}\right)
$$

A measured EBL intensity in the range $43-175 \mathrm{nW} \mathrm{m}^{-2} \mathrm{sr}^{-1}$ implies that $\sim 2 \%$ of the original Hydrogen content of the universe has been used over cosmic time to power the nuclear reactions inside stars [107].

\subsubsection{The connection between Radio and IR backgrounds}

As explained in section 2.2.1.1, the H II regions around hot massive stars are strong emitters of free-free (bremsstrahlung) radiation at IR and radio wavelengths, establishing a correlation between the IR and radio emission from star-forming galaxies that has been confirmed by observations ${ }^{11}[110]$.

Using this correlation it has been calculated that $50 \%$ of the Cosmic Radio Background $(\mathrm{CRB})$ at $170 \mathrm{~cm}$ is originated in star-forming galaxies, while the integrated flux from discrete AGN-like radio sources accounts for $50 \%$ of the CRB at $75 \mathrm{~cm}$.

\footnotetext{
${ }^{10}$ This is obviously a crude approximation. Hauser and Dwek in [107] assume that all Hydrogen burning occurred in a single burst of star formation at $z \sim 1$. They find $\rho_{\Delta \mathrm{X}}$ that is twice the value implied in eq. 2.11, which is still less than the uncertainty in $I_{\mathrm{EBL}}$.

${ }^{11}$ The radio emission of AGNs is due to a different physical process, therefore such correlation is not expected, nor has been observed.
} 
Both contributions suggest a consistent and complete model for the origin of the CRB [102].

\subsection{EBL Models}

\subsubsection{Description of EBL Models}

As discussed in the previous section, the EBL intensity at the present epoch $(z=0)$ and its measurements provide an integral constraint on the history of energy releases in the universe. However, specific issues like the evolution of star and element formation cannot be addressed by measuring the cumulative energy output only. That is why several approaches have been developed to calculate the EBL density $n(\epsilon, z)$ as a function of redshift from astrophysical principles. The models encompass different degrees of complexity, observational constraints and data inputs. A brief description of the different categories of models is presented below (see [107] for a complete review). Sections 2.5.2.1 through 2.5.2.3 summarize the most recent EBL models and their results.

\subsubsection{Simple Backward Evolution Models}

Backward evolution models extrapolate the spectral properties of local galaxies to higher redshifts using a parametric model for their evolution. In these models, the EBL luminosity density is given by the convolution of the galaxy luminosity function with the galaxy spectral luminosity (SED), summed over galaxy types. Furthermore, $n(\epsilon, z)$ is calculated separately at UV-optical and infrared wavelengths because different physical processes are involved. Evolution is introduced through pure luminosity evolution (scaling of galaxy spectra with redshift), or as pure density evolution (change in the comoving number density with redshift).

Backward evolution models are simple and they can be easily compared with ob- 
servations thanks to their predictions regarding number-magnitude, number-redshift, and color-magnitude relations for galaxies. Nevertheless, they do not account for processes known to occur in galaxies, such as star and metal formation, and re-emission of radiated power by dust (see [107] for a review of the models).

\subsubsection{Forward Evolution Models}

Forward evolution models predict the temporal evolution of galaxies and their emissivity spectrum from astrophysical principles. Every forward evolution model relies on a spectral evolution program that follows the evolution of stellar populations while calculating the stellar, gas, and metallicity content of a galaxy and its resulting SED as a function of time starting at the onset of star formation [107]. The astrophysical routines and data sets required by these models include:

- Stellar evolutionary paths (with chemical composition).

- Libraries of calculated and observed stellar atmospheres.

- Effects of dust on the scattering, absorption and thermal re-radiation of starlight.

- Evolution of dust abundance, composition, size and distribution with respect to the emitting sources.

All these processes are then embedded in a cosmological model determined by $H_{0}, \Omega_{\mathrm{M}}$ and $\Omega_{\Lambda}$. The free parameters of the model are then adjusted to match the observed galaxy number counts, SEDs, colors and metallicity at the different redshifts.

Forward evolution models have been proven successful in reproducing the general characteristics of the observed EBL (as expected, given their multitude of freeparameters). However, these models lack the capability to account for galaxy interactions, starburst episodes or morphological evolution of galaxies. In particular, forward evolution models fail to predict the observed ULIRGs (Ultraluminous Infrared Galaxies) discussed in section 2.2.1.2. 


\subsubsection{Semi-analytical Models}

To overcome the shortcomings of forward evolution models, semi-analytical models (SAMs) have been developed to reproduce in simulations the process of structure formation, providing a physical model for the formation and evolution of galaxies.

In addition to the normal quiescent cosmic star formation in galactic disks, SAMs account for the stochastic starbursts resulting from galaxy interactions or merging events. To achieve this, SAMs include numerical routines for the cooling of gas that falls into halos, and for the star formation efficiency during merger events.

The underlying causes of discrepancy between predictions from the models and observations are hard to identify. When one considers the approximations used to describe the different physical processes, plus the uncertainties in the observational data used as input, and the fundamental shortcomings innate of a simulation, it is not surprising to find some discrepancies.

\subsubsection{Chemical Evolution Models}

Chemical evolution models deal with the average properties of the universe rather than trying to account for the complex processes that determine how galaxies form, shine and evolve. Chemical evolution models provide a picture for the evolution of the mean density of stars, interstellar gas, metals and radiation averaged over the entire population of galaxies. Data inputs to these models trace the stellar activity and properties of the ISM. Typical inputs include the mean rest-frame UV emissivity as determined by deep surveys, and the contents of the ISM as determined by quasar absorption lines. Spectral synthesis models are used then to calculate the EBL density due to starlight at every redshift. Chemical evolution models have been able to reproduce the spectral shape of the EBL and other numerous observations (see [107] and references therein). 


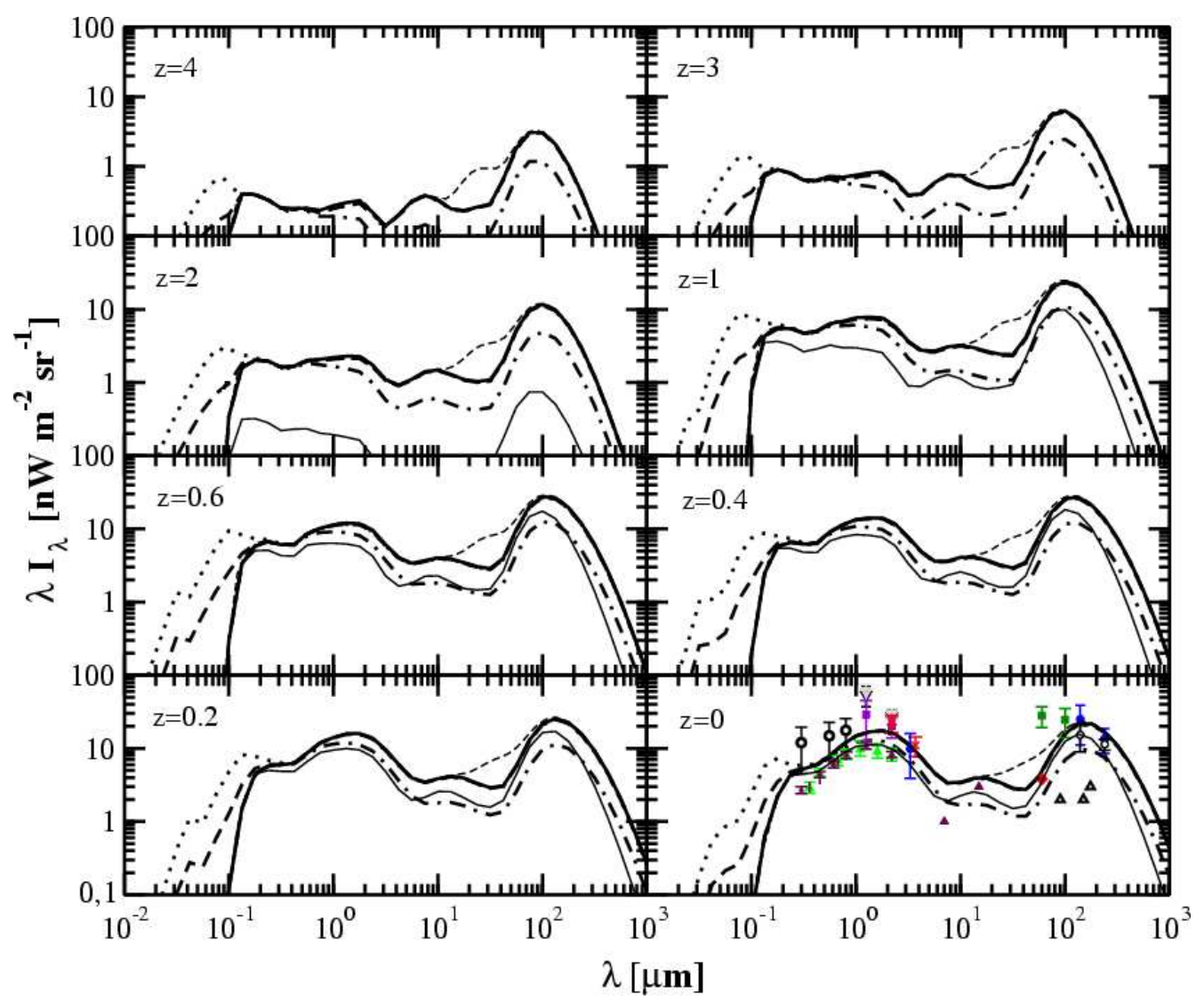

Figure 2.6: EBL density at various redshifts, adapted from [144]. Best-Fit model (thick solid line); Warm-Dust model (thin dashed line); Low-IR model (dot-dashed line); Low-SFR model (thin solid line); Stellar-UV model (dashed line); and Highstellar- $U V$ model (dotted line). Data points at $z=0$ are obtained from direct measurements of the EBL (see section 2.3).

\subsubsection{EBL models used for GLAST Simulations}

\subsubsection{Kneiske et al (2004)}

Kneiske et al [144] treat the EBL-modeling problem with different approaches at UV-optical and infrared wavelengths. For the UV-optical part they use a chemical evolution model, while backwards evolution is used for the infrared. In particular, their infrared model takes into account data from deep galaxy surveys, consistent with a dust-rich universe at high redshifts.

A very useful feature of the model by Kneiske et al is the parametrization of the EBL density in terms of the main observational uncertainties, including: i) the 
redshift dependence of the cosmic star formation rate, and ii) the fraction of UV radiation released from star forming regions. The EBL model by Kneiske et al comes then in different flavors (see fig. 2.6) that are used to bracket the available data from direct EBL measurements.

- The Best-Fit model is the one that best interpolates the data. Total UV absorption by interstellar gas is assumed shortwards of $0.1 \mu \mathrm{m}$.

- For the Warm-Dust model the different dust contributions are calculated to fit the line intensities detected by IRAS at 12, 25, 60 and $100 \mu \mathrm{m}$ (see section 2.3.2.5)

- The Low-IR model is set to the minimum infrared EBL flux as determined from integrated galaxy counts. Together with Warm-Dust, these two models bracket the infrared segment of the EBL.

- The Low-SFR model allows for a steep decline of the SFR at high redshifts, a matter of current debate as discussed in section 2.4.2.

- In the Stellar-UV model all the UV radiation produced by the stellar populations escapes to the intergalactic medium after reprocessing by the interstellar gas.

- Finally, the High-Stellar-UV model allows for a strong UV-field at high redshifts. Together with Best-Fit, these two models bracket the EBL density at UV wavelengths.

Since the $\gamma$-ray sources that will be observed with GLAST are particularly sensitive to the EBL density at UV wavelengths, the Best-Fit and High-Stellar-UV models are used in the simulations described in this dissertation to bracket the possible ranges of attenuation. 


\subsubsection{Primack et al}

Primack and collaborators $[222,223]$ have pioneered the use of SAMs described in section 2.5.1.3. The most recent iteration of their model [222] has benefited from recent measurements of the local luminosity density at optical and NIR wavelengths and a well established cosmological model. The key parameters in their model (those that govern the rate of star formation, supernova feedback and metallicity) have been adjusted to fit the local galaxy data. It is fair to say, however, that this model is not able to account [58] for the bright galaxies observed with SCUBA at far infrared wavelengths [24].

With respect to their estimates from previous years, the most recent version of the model yields a lower luminosity density at optical wavelengths, resulting in a reduced EBL density. Recent $\mathrm{TeV}$ observations of nearby blazars seem to support such low values (section 2.6.4.1).

\subsubsection{Stecker et al}

Stecker et al have made key contributions to the field of EBL modeling with their backwards evolution models. As new data have become available, their EBL model has gone through different iterations: Malkan \& Stecker (1998) [168], Salamon \& Stecker (1998) [233], Malkan \& Stecker (2001) [167], and the most recent Stecker et al (2006) [253]. All versions share roughly the same approach and new results are used to improve and test the model.

In the most recent model, Stecker et al calculate the EBL at infrared and opticalUV wavelengths separately. At infrared wavelengths, they use a backwards evolution model based on observational knowledge of: (i) luminosity dependent galaxy SEDs, (ii) galaxy luminosity functions, and (iii) parametrized functions for luminosity evolution. The first item is the most crucial and controversial [222] since it establishes that the SED of a galaxy can be predicted, at least statistically, from its observed 
luminosity at a given wavelength. Once that piece is in place, it is straightforward to calculate the overall energy release at any redshift.

For optical-UV wavelengths, Stecker et al consider the redshift evolution of stellar populations with an analytical approximation to the more sophisticated SEDs used in Salamon \& Stecker (1998) [233]. The SEDs adapted from [38] reflect stellar population synthesis models for galaxy evolution and the observational fact that star forming galaxies are "bluer" (brighter in the blue part of the optical spectrum) at $z>0.7$. It

should be noted for this model that: (i) the UV spectra for all SEDs are assumed to cut off at the Lyman limit, and (ii) the effects of extinction by dust are not included. The former is a matter of debate since it is not really known how much UV radiation shortwards of the Lyman limit can leak out from star forming regions. The latter leads to a large UV photon density, and thus, to strong gamma-ray opacity at high redshifts.

\subsection{EBL attenuation of gamma-ray sources}

\subsubsection{Historical Background}

The potential absorption in an astrophysical context of high energy photons by pair production reactions was first pointed out by Nikishov in 1962 [204]. After the discovery of the CMB in the sixties, Gould \& Schreder [98] and Jelley [125] predicted that the universe is opaque to $\gamma$-rays of energy above $100 \mathrm{TeV}$ from extragalactic sources. Fazio and Stecker [80, 258] calculated the cosmological and redshift effects on the attenuation, predicting that photons emitted at redshift $z$ with energy above $\sim 100 /(1+z)^{2} \mathrm{TeV}$ would be strongly absorbed by the CMB. However, Greisen in 1968 [100] was the first to actually suggest that pair-production with optical photons 
(instead of CMB) at $z \sim 10$ would result in a spectral cut-off ${ }^{12}$ around $\sim 10 \mathrm{GeV}$ in the $\gamma$-ray flux.

With the discovery of bright extragalactic $\gamma$-ray sources by CGRO (section 1.1.3), Stecker, de Jager and Salamon [257] proposed the use of absorption features in the spectrum of blazars to determine the intensity of the the cosmic infrared background, provided that the newly EGRET-discovered blazars emitted $\gamma$-rays up to TeV energies. The subsequent discovery of a handful of TeV-emitting blazars by ground-based instruments stimulated the calculation of upper limits on the infrared background (section 2.6.4.1).

\subsubsection{Calculation of the Optical Depth}

EBL attenuation is a function of the observed $\gamma$-ray energy $E$ and the redshift $z$ of the emitting source. The attenuation is generally parametrized by the optical depth $\tau(E, z)$, which is defined as the number of e-fold reductions of the observed flux $\mathcal{F}_{\text {obs }}$ as compared with the emitted source $\mathcal{F}_{\text {emitted }}$ at redshift $z$ [26]:

$$
\mathcal{F}_{\text {obs }}=e^{-\tau(E, z)} \mathcal{F}_{\text {emitted }}
$$

The optical depth is calculated from physical principles. Using the cross section introduced in section 1.1.1.3, and assuming isotropic background radiation with spectral density $n(\epsilon)$ at energy $\epsilon$, the absorption probability of $\gamma$-rays per unit path is given by

$$
\frac{d \tau}{d l}=\int_{0}^{2 \pi} \sin \theta d \theta \int_{\epsilon_{t h}}^{\infty} n(\epsilon) \sigma(E, \epsilon, \theta) d \epsilon
$$

where $\theta$ is the scattering angle for the $\gamma-\gamma$ collision, $\epsilon_{t h}=\frac{2 m^{2} c^{4}}{E(1-\cos \theta)}$ is the energy threshold for the reaction, and $m$ is the electron mass. Since blazars and other cosmic sources are being considered, redshift is a good choice to measure the distance, with

\footnotetext{
${ }^{12}$ Energy cut-off is defined as the energy where the $\gamma$-ray flux has been attenuated by $e^{-1}$.
} 
the total distance being the look-back time (times the speed of light $c$ )

$$
\begin{aligned}
L & =\int_{0}^{z} d z \frac{d l}{d z} \\
& =\int_{0}^{z} d z \frac{c}{H_{0}(1+z)}\left[(1+z)^{2}\left(1+\Omega_{M} z\right)-z(2+z) \Omega_{\Lambda}\right]^{-1 / 2}
\end{aligned}
$$

where $H_{0}, \Omega_{M}$, and $\Omega_{\Lambda}$ are the cosmological parameters already introduced in section 2.4 .2 .

Using the expressions above, the optical depth can be written as a function of the observed energy $E$ and the redshift of the emitting source

$$
\begin{aligned}
\tau(E, z) & =\int_{0}^{L} \frac{d \tau}{d l} d l=\int_{0}^{z} d z^{\prime} \frac{d l}{d z^{\prime}} \frac{d \tau\left(E^{\prime}, z^{\prime}\right)}{d l} \\
& =\int_{0}^{z} d z^{\prime} \frac{d l}{d z^{\prime}} \int_{0}^{2 \pi} \sin \theta^{\prime} d \theta^{\prime} \int_{\epsilon_{t h}^{\prime}}^{\infty} d \epsilon n\left(\epsilon^{\prime}, z^{\prime}\right) \sigma\left(E^{\prime}, \epsilon^{\prime}, \theta^{\prime}\right)
\end{aligned}
$$

where the primed variables $\left(E^{\prime}, \epsilon^{\prime} n\left(\epsilon^{\prime}, z^{\prime}\right), \theta^{\prime}\right)$ refer to the values calculated in the comoving frame at $z=z^{\prime}$. Thus, the optical depth depends on three distinct physical quantities:

- The spectral energy density of EBL photons as a function of redshift, $n(\epsilon, z)$.

- The cosmological line length determined by $H_{0}, \Omega_{\mathrm{M}}$, and $\Omega_{\Lambda}$.

- The $\gamma-\gamma$ cross section.

The spectral energy density of EBL photons is the most poorly measured of the three and the focus of this dissertation is precisely to probe the EBL by measuring the attenuation of $\gamma$-rays. Next, the cosmological parameters have been measured accurately in the recent years with data from WMAP [249, 250]. Finally, the $\gamma-\gamma$ cross section is in principle well understood with experimental confirmation to a very high precision. There exists, however, the possibility of "exotic" corrections to the 
cross section that are highly conjectural, as described below. Although none of these corrections have been observed, high energy $\gamma$-rays traversing cosmological distances probe a region of the phase-space of fundamental physics that is not usually accessible in the lab $[123,254]$. So, apart from the cases considered below, for the remainder of this document it is assumed that the cross section is given by the standard-model expression presented in equation 2.16 .

\section{"Exotic" corrections to the pair-production cross section}

The existence of a new, light particle ${ }^{13}$, pair-produced via $\gamma-\gamma$ inelastic scattering, would add a new channel to the reaction and in consequence, would increase the scattering cross section [26]. No well-known extension of the Standard Model predicts an alternative light and final-state particle whose existence has not been already been excluded by the current accelerators.

A more interesting possibility for exotic phenomena is Lorentz invariance violation ([124] and references therein). Although such violation is common in different approaches to a quantum theory of gravity [9], its strength and energy scale remain debatable. Even though the effects should be small ${ }^{14}$, the cumulative effects after traversing cosmological distances could be detectable. A violation of Lorentzinvariance would change the threshold condition for pair production to an extent that depends on the quantum-gravity properties of the universe [27].

\footnotetext{
${ }^{13}$ The reaction $\gamma+\gamma \rightarrow f+\bar{f}$ is allowed for any fermion-antifermion pair as long as the the reaction is kinematically possible (energy threshold). Nevertheless, the cross section is proportional in leading order to $\sim 1 / m_{f}^{2}$ [104], hence reactions that involve heavy-fermions $(\mu, \tau, \ldots)$ contribute a negligible amount.

${ }^{14} \mathcal{O}\left(E / E_{Q G}\right)$ for a $\gamma$-ray with energy $E$, where $E_{Q G}$ is the assumed energy scale for quantum gravitational effects which can couple to electromagnetic radiation. Although $E_{Q G}$ is often assumed to be in the order of the Planck scale $\left(E_{P} \simeq 10^{19} \mathrm{GeV}\right)$ or extra dimensions, work within the context of string theory suggests that quantum gravity effects can be noticeable at energies below the Planck scale and perhaps as low as $10^{16} \mathrm{GeV}$ [277].
} 


\subsubsection{Optical Depth calculated from EBL Models}

If the EBL density is known, it is straightforward to calculate the optical depth $(\tau)$ to $\gamma$-rays according to the expressions introduced above. The EBL models introduced in section 2.5.2 have been published with their corresponding optical depths expressed in function of the observed $\gamma$-ray energy $E$ and redshift of the emitting source $z$. Figures 2.7 and 2.8 show $\tau(E, z)$ according to four EBL models used for GLAST simulations: (i) Kneiske - Best Fit, (ii) Kneiske - High UV, (iii) Primack - 2005, and (iv) Stecker - 2006. Influenced by GLAST's energy range, the plots indicate the optical depth to photons with observed energy $1<E<500 \mathrm{GeV}$.

\section{Gamma-ray Horizon}

For an observed gamma-ray energy $E$, the gamma-ray horizon is defined as the source redshift $z$ for which the optical depth is $\tau(E, z)=1$. Therefore, the gamma-ray horizon gives the redshift of a source for which the intrinsic flux at energy $E$ suffers an e-fold absorption when observed at $z=0$ due to the EBL. The $\gamma$-ray spectrum of a source outside the horizon suffers severe attenuation above the energy $E$.

The relation $\tau(E, z)=1$ is very useful to study the EBL attenuation of $\gamma$-rays and has been coined [144] the "Fazio-Stecker relation" (first shown in [80]). Figure 2.9 shows the Fazio-Stecker relation predicted by the models described in section 2.5.2. A Fazio-Stecker plot can be used to compare measured EBL-attenuation with theoretical expectations. In Chapter 5 this approach is used with detailed Monte Carlo simulations of gamma-ray sources to be observed with GLAST.

The following can be inferred from close inspection of the plots in figures 2.7-2.9:

- The models share the same qualitative features, but quantitatively they differ significantly. 

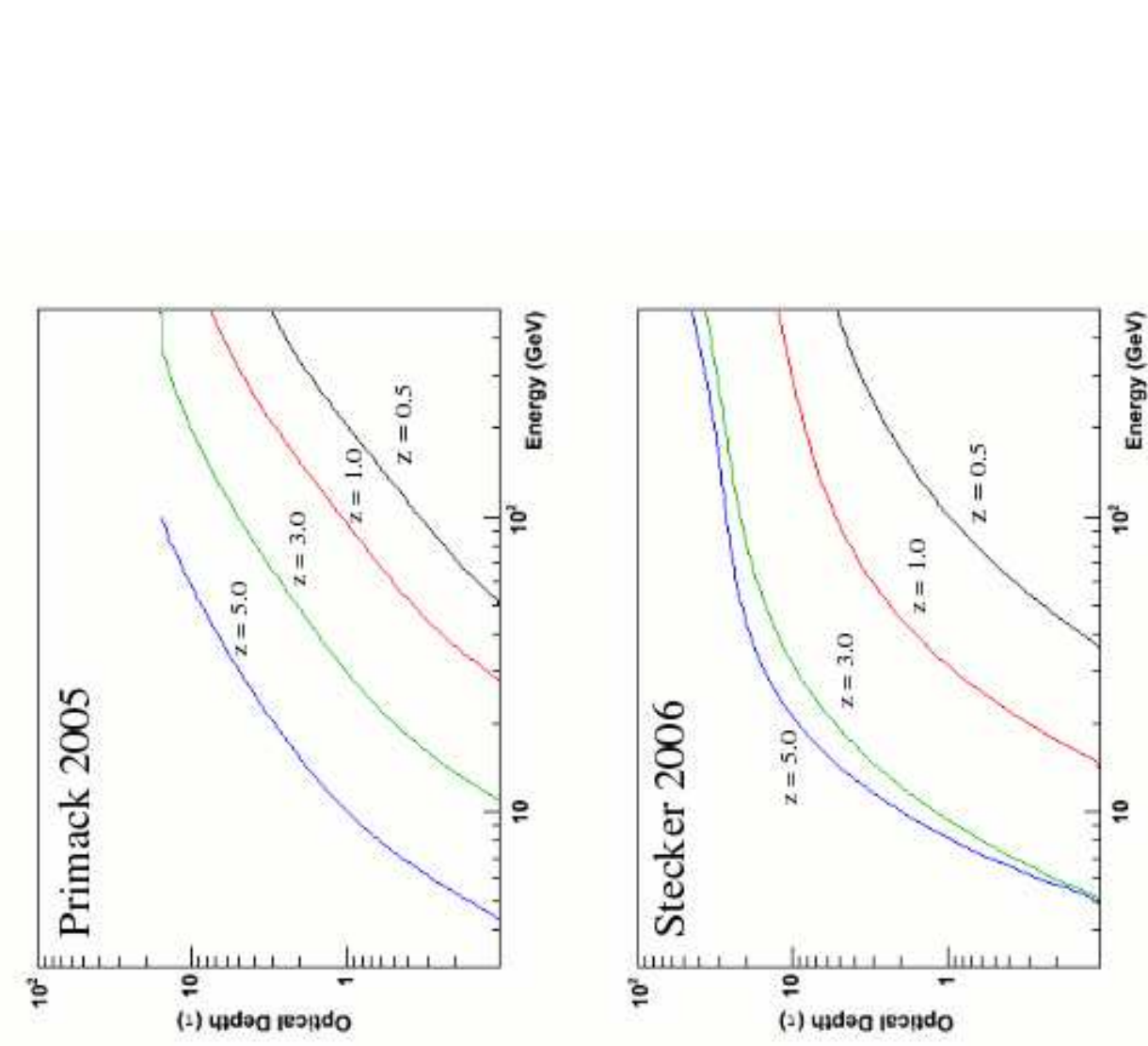

워

ชิ

10

क ज

苞

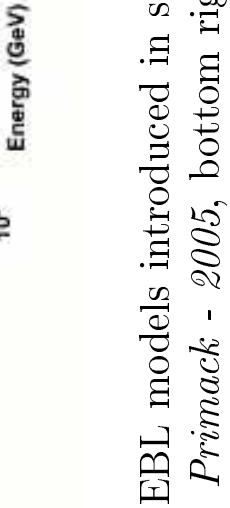

获

$\circ$

so :

$\exists$

-

$\approx$

过
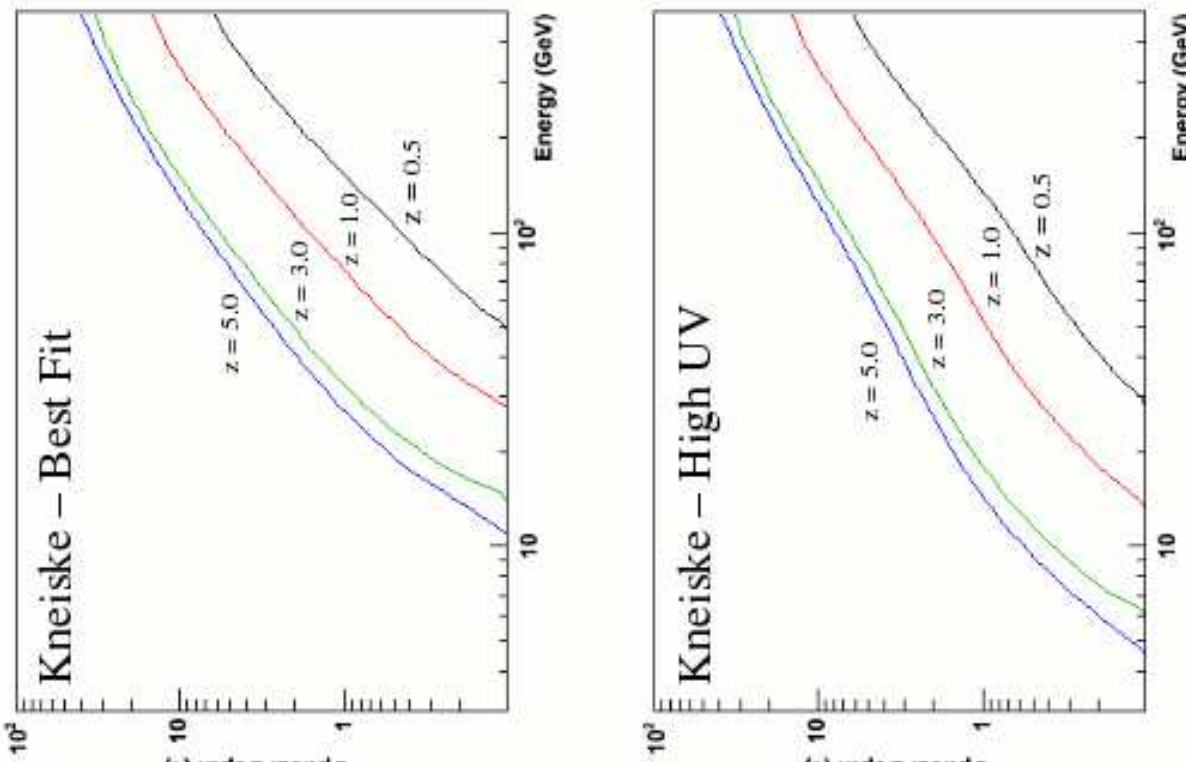

5.

च

$00 . \frac{\pi}{2}$

है

岁过

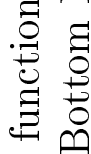

$\pi$

路

華

ซึ่

苟 む

‥

ㄱ

$0 \leqslant$

焉通 

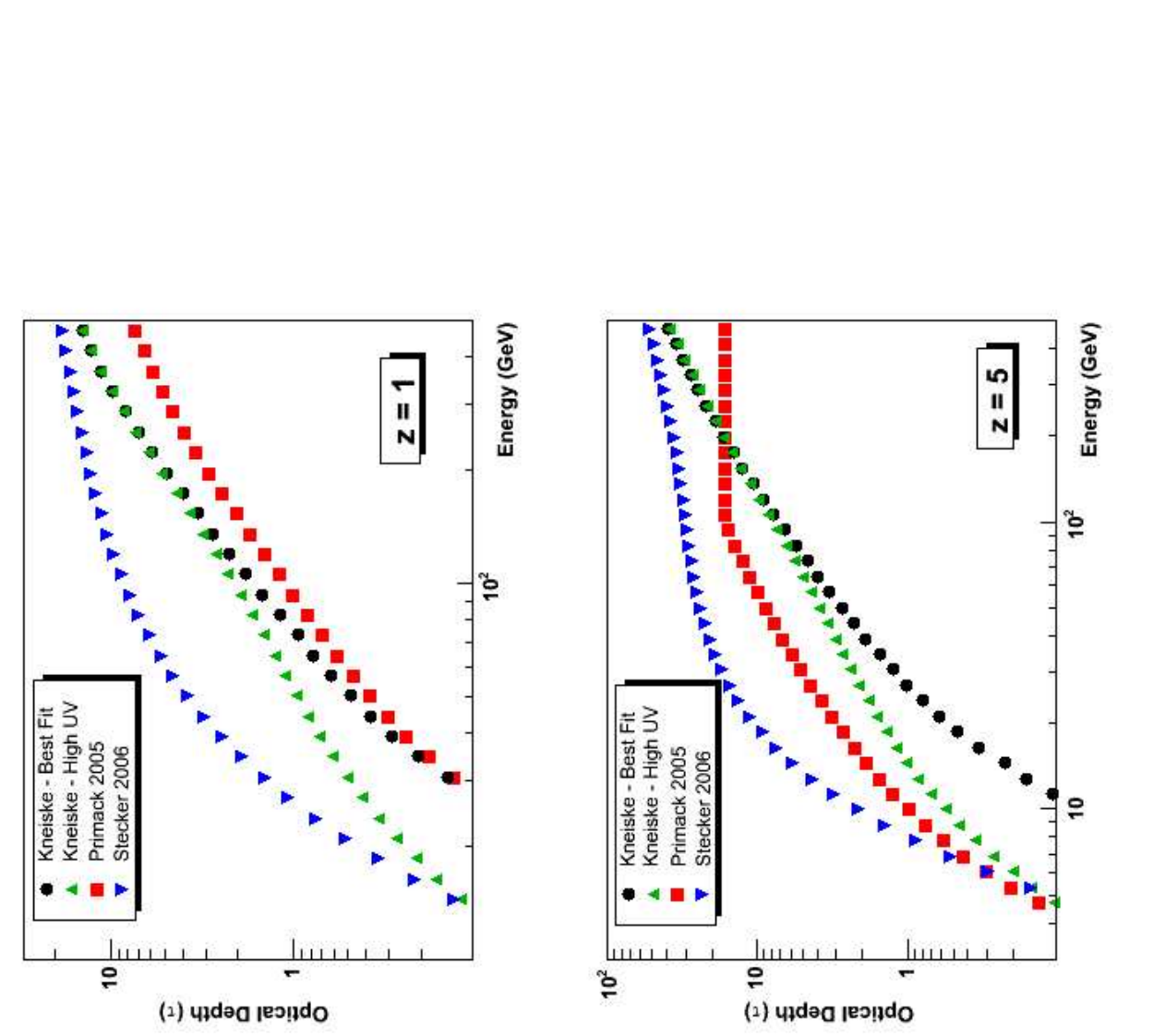

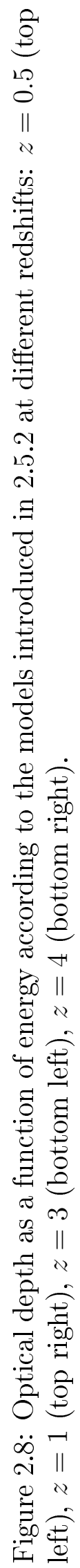

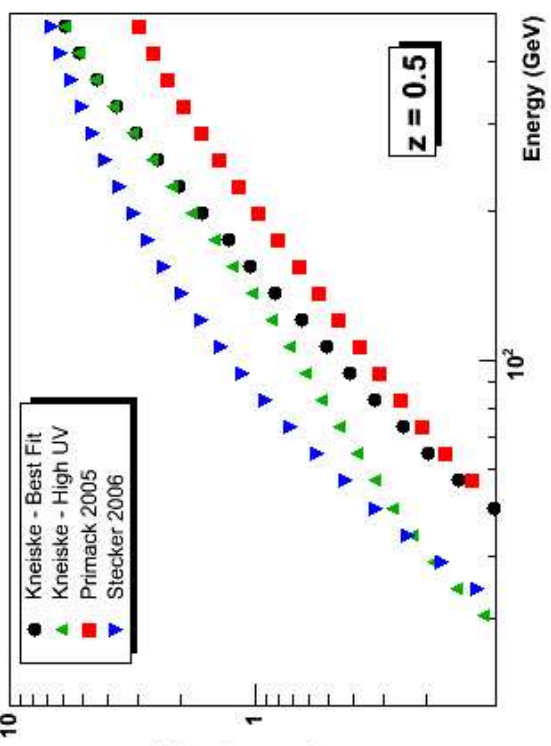

(2) पłdəa ןeo!̣do

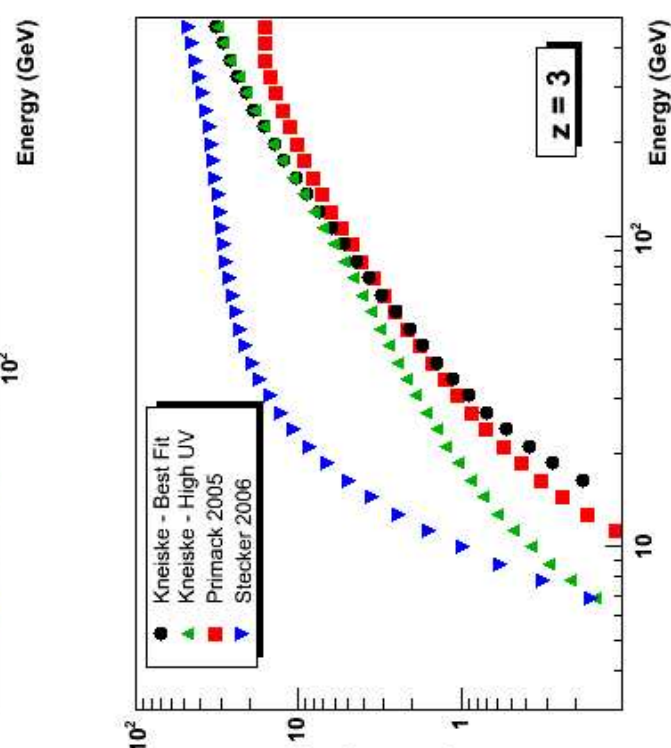

(1) पұdea ןeon!do

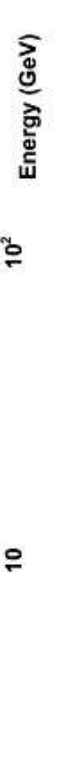


- The universe is optically thin $(\tau<1)$ to $\gamma$-rays with energy below $\sim 10 \mathrm{GeV}$, independently of the model. This is due to the rapid extinction of EBL photons shortwards of the Lyman limit. If there were a bright far-UV or X-ray diffuse background ${ }^{15}$, then sub-GeV $\gamma$-rays would suffer a similar attenuation.

- At low and moderate redshifts $z \lesssim 2$, the Primack - 2005 model (section 2.5.2.2) predicts the least amount of attenuation. Interestingly, for sources at high redshifts the optical depth continues to increase rapidly in this model (at $z \sim 5$, Primack - 2005 is not the model predicting least attenuation).

- At moderate and high redshifts $(z \sim 1-5)$, the optical depth for $\gamma$-rays in the $\mathrm{GeV}$ regime is dominated by the $\mathrm{UV}$ part of the EBL. In consequence, the cut-off energy decreases when the UV density is high, and vice versa. The UV density at high redshifts in turn is dominated by the SFR (especially at high redshifts) and dust-extinction effects, which are not well constrained. This is evident from the plots: the cut-off energy for a source at $z=1$ ranges between $\sim 30 \mathrm{GeV}$ (Stecker - 2006) and $\sim 100 \mathrm{GeV}$ (Primack - 2005). Measurement of the EBL attenuation of gamma-ray sources at cosmological distances is needed for a better understanding of the SFR and dust extinction.

- The Stecker - 2006 model predicts more absorption at high redshifts than the other models. This can be attributed in part to the omission in the model of extinction effects of UV photons by the interstellar gas in galaxies (section 2.5.2.3)

- Gamma-ray instruments with a threshold much lower than $\sim 100 \mathrm{GeV}$ are required to measure the cut-off energies for sources located at cosmological

\footnotetext{
${ }^{15}$ The cosmic X-ray background (CXB) has a peak intensity of $47 \pm 0.5$ (stat) \pm 1.5 (sys) $\mathrm{keV}^{-2} \mathrm{~cm}^{-2} \mathrm{~s}^{-1} \mathrm{keV}^{-1} \mathrm{sr}^{-1}$ in the energy range $0.1 \mathrm{keV}-100 \mathrm{keV}$ [45]. The integrated CXB intensity $\left(\sim 10^{-2} \mathrm{nW} \mathrm{m} \mathrm{m}^{-2} \mathrm{sr}^{-1}\right)$ is thus about four orders of magnitude lower than the integrated EBL intensity $\left(\sim 10^{2} \mathrm{nW} \mathrm{m}{ }^{-2} \mathrm{sr}^{-1}\right.$, as discussed in section 2.4.1).
} 
distances $z \gtrsim 1$, where the effects of SFR and dust-extinction are relevant.

- Gamma-ray instruments with a threshold below $\sim 10 \mathrm{GeV}$ have access to gamma-ray sources at any redshift ${ }^{16}$ (provided their flux is above the instrument's sensitivity).

\subsubsection{Using gamma-ray sources to measure the EBL density}

All the preliminary information is now in place to discuss how to use the attenuation of gamma-ray sources to measure the effects of the EBL flux on the spectra of extragalactic $\gamma$-ray sources. The main handicap when using this approach is the uncertainty about the intrinsic spectrum of the gamma-ray source before the absorption has taken place. Emission mechanisms of blazars are not completely understood, therefore it is not possible to predict with certainty the intrinsic spectrum a particular source has, even when simultaneous multiwavelength observations are available. Furthermore, the presence of optical-infrared radiation fields within the source [64] could result in spectral cut-offs that are completely independent of any cosmic attenuation effect. It is argued in this dissertation that these obstacles may be overcome by measuring the energy cut-offs of a large sample of sources at different redshifts (provided a sufficiently large population of sources is observationally accessible), and by differentiating the EBL-induced spectral features (which correlate with redshift) from those that are peculiar to the sources.

\subsubsection{Attenuation of TeV sources by IR-EBL}

So far, only $\mathcal{O}(\gtrsim 100 \mathrm{GeV})$ observations of BL Lacs have been available in order to look for EBL attenuation effects (Mrk 421, Mrk 501, 1ES 2344+514, 1ES 1959+650,

\footnotetext{
${ }^{16}$ At $z \sim 200$ the universe becomes optically-thick to $\gamma$-rays with $E \lesssim 10(1+z) \mathrm{GeV}$ due to pair production on atoms, ions and free electrons [281].
} 


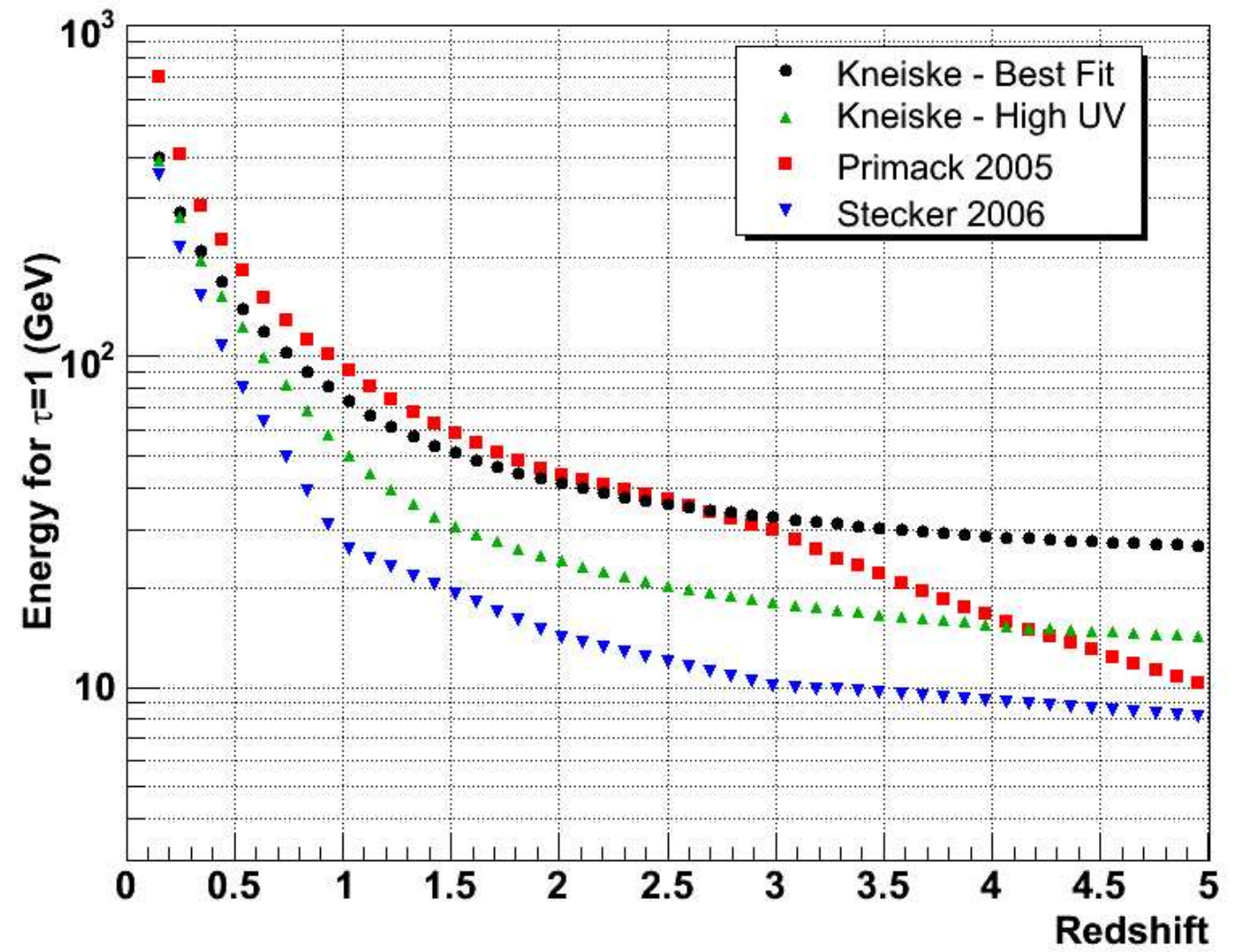

Figure 2.9: Fazio-Stecker relation for the EBL models used in GLAST simulations. 


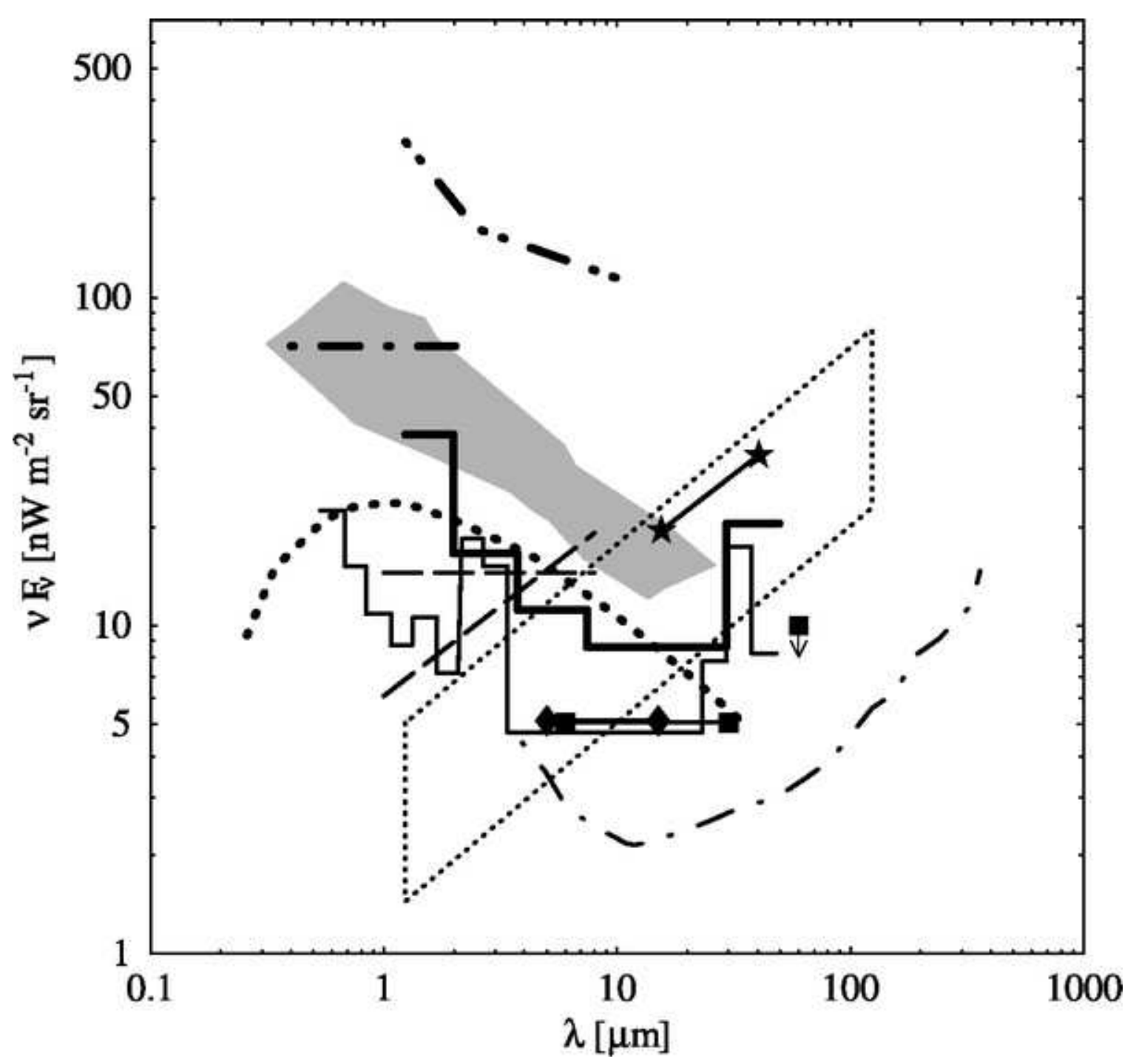

Figure 2.10: Compilation (as of 2005) from [242] of EBL upper limits derived from observations of TeV blazars. Stecker \& de Jager [256] (dashed line), Dwek \& Slavin [72] (line with stars), Biller et al. [23] (thick double-dot-dashed line), Funk et al. [89] (thin dot-dashed line), Stanev \& Franceschini [252] (thin solid line), Biller et al. [22] (thick solid line), Guy et al. [101] (thick dot-dashed line), Vassiliev [271] (thick dotted line), Dwek [69] (line and single point with squares), Renault et al. [228] (line with diamonds), Schroedter [242] (gray region, 98\% confidence level). The dotted parallelogram shows the EBL flux estimate of de Jager et al. [54]. All flux values have been rescaled to $\mathrm{H}_{0}=71 \mathrm{~km} \mathrm{~s}^{-1} \mathrm{Mpc}^{-1}$. 
PKS 2155-413 and H1426+428). These observations have been used to derive upper limits to the local EBL at infrared wavelengths that are presented in figure 2.10. Four approaches have been used [242]:

1. An emission model is used to predict the intrinsic spectrum through fitting of multiwavelength data. A spectral EBL shape is then assumed with only the absolute normalization left as a free parameter. The EBL intensity is then estimated by fitting the model to the observed $\gamma$-ray flux $[23,54,72,101,257$, $256]$.

2. The deviation, or lack thereof, of the data from an assumed intrinsic spectrum (e.g. a power law) is used to place an upper limit on the EBL density, which again has been assumed to have a predefined spectral shape [22, 89, 252, 271].

3. For a given EBL model (usually with free normalization) the intrinsic source spectrum is reconstructed from the observed data. If the reconstructed spectrum rises exponentially, or is inconsistent with the synchrotron peak, or has any other "unphysical" behavior, the used EBL model is ruled out, or an upper limit is placed on its normalization [2, 6, 68, 69, 101, 148, 228].

4. The fact that for a given gamma-ray energy $E_{\gamma}$ there is an EBL wavelength $\lambda_{0}$ for which the cosmic absorption is maximum was cleverly used by Schroedter [242] to place an upper limit on the EBL density at discrete wavelengths without having to assume an EBL shape. If one calculates the spectral EBL density $n\left(\lambda_{0}\right)$ assuming that the total attenuation at energy $E_{\gamma}$ is due exclusively to a monochromatic EBL with wavelength $\lambda_{0}$, then $n\left(\lambda_{0}\right)$ becomes a conservative upper limit to the EBL at wavelength $\lambda_{0}$ (since a higher density would result necessarily in a greater absorption than the one observed). 
Low level EBL suggested by HESS observations of two BL Lacs at $\langle\mathbf{z}\rangle \sim 0.18$

The recent discovery of gamma-ray emission with hard spectra from the BL Lacs $\mathrm{H}$ 2356-309 $(z=0.165)$ and 1ES 1101-232 $(z=0.186)$ by the HESS collaboration [2] suggests an upper limit to the EBL at optical-NIR wavelengths that is very close to the lower limit given by the integrated light of resolved galaxies (section 2.3.1). This implies that the universe is more transparent to high energy $\gamma$-rays than previously thought and that a significant contribution from sources other than starlight is excluded.

Figure 2.11 shows the observed (red squares) and absorption-corrected spectrum (blue squares) of 1ES 1101-232 according to the EBL realizations presented in figure 2.12, where P1.0, P0.55, and P0.45 correspond to the assumed EBL shape scaled respectively to $100 \%, 55 \%$ and $45 \%$. In particular, $\mathrm{P} 1.0$ is in general agreement with the EBL spectrum expected from galaxy emission [222]. $\mathrm{E}_{\mathrm{NIR}}$ is the excess contribution around $1.5 \mu \mathrm{m}$ detected with IRTS (section 2.3.2.3).

The correction of the observed data due to absorption ${ }^{17}$ results in an "unusual" spectrum (defined by [2] as any power law $\left(E^{-\alpha}\right)$ with $\left.\alpha<1.5\right)$ if the base spectral shape is scaled by any factor over $45 \%$ ( $55 \%$ to allow for evolutionary effects). Furthermore, any EBL realization that includes the NIR excess $E_{\text {NIR }}$ yields an "unusual" spectrum, which suggests that the full NIR excess (interpreted by some as redshifted radiation from Pop III stars) if real, is not entirely extragalactic ${ }^{18}$.

The short dashed line in figure 2.12 shows the additional UV component needed in addition to P1.0 to yield a "usual" intrinsic spectrum. In such case, the EBLabsorption-corrected spectrum around $\sim 200 \mathrm{GeV}$ (low-energy data points in fig. 2.12) would significantly go up in flux and thus, the intrinsic spectrum satisfies $\alpha>1.5$.

\footnotetext{
${ }^{17}$ Due to the low redshift of the blazars, no evolution of the EBL is assumed.

${ }^{18}$ The HESS results however, do not exclude all levels of NIR excess. Modest, although significant flux levels are still allowed [133].
} 


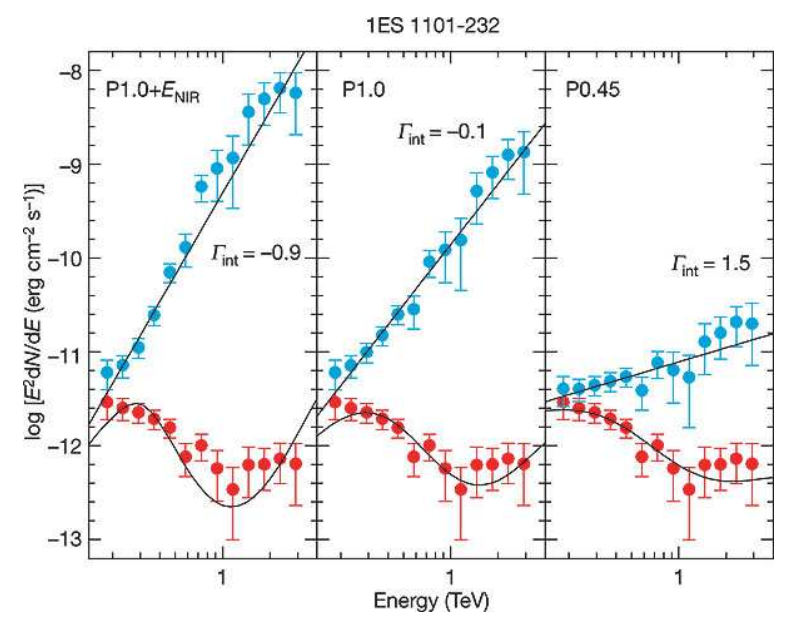

Figure 2.11: The observed (red) and corrected (blue) spectra of 1ES 1101-232 [2]. The observed spectra has been corrected for absorption with three different EBL spectral shapes as explained in the text. The lines show the best-fit power law to the corrected spectrum.

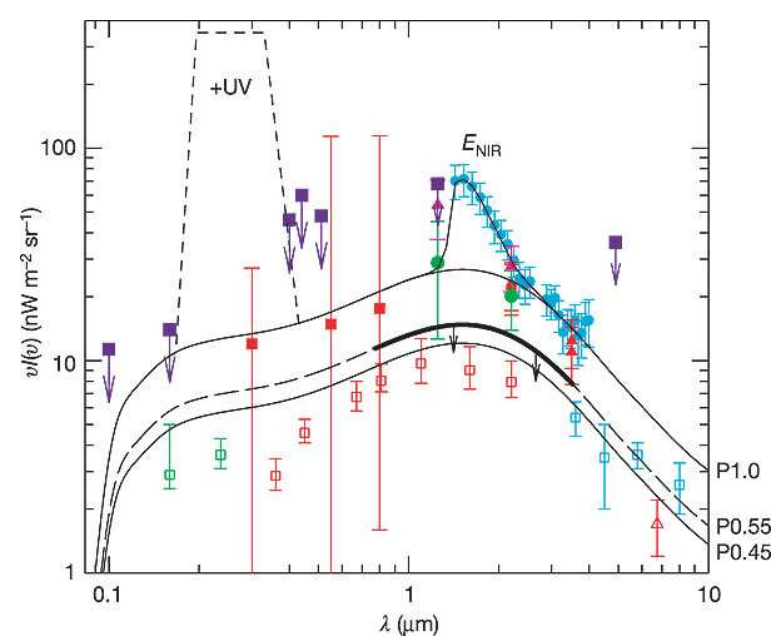

Figure 2.12: Limits on the spectral energy distribution of the EBL [2]. The data points correspond to the direct measurements discussed in section 2.3. In particular, the open symbols correspond to the integrated light from galaxy counts, and thus should be considered as a lower limit to the EBL. The curves show the EBL shapes used to reconstruct the intrinsic spectra in figure 2.11 where P1.0, P0.55, P0.45 are the absolute normalizations of the assumed EBL spectral shape $(100 \%, 55 \%$ and $45 \%$ respectively). The thick line in the $\mathrm{P} 0.55$ curve shows the range most effectively constrained by the data. The short dashed line shows the additional UV component needed in addition to P1.0 in order to yield a "physical" intrinsic spectrum for the blazars. 
Therefore, it is still possible to have an EBL flux at optical and NIR wavelengths that is significantly above the integrated light from galaxy counts, but this requires the EBL-UV flux in the $0.15-0.3 \mu \mathrm{m}$ range to be well above the level suggested by the upper limits discussed in section 2.3.1.

In summary, given the assumptions outlined above (blazars with intrinsic spectral index $\alpha<1.5$ are unphysical, and the EBL flux at UV wavelengths conforms to the upper limits available from observations) the HESS results provide a strong limit on the EBL flux for wavelengths below $2 \mu \mathrm{m}[133]$, and in particular, they exclude an extragalactic NIR excess at the level measured by DIRBE and IRTS (section 2.3.2) at those wavelengths.

Extension of the observed spectrum of these blazars to energies below $\sim 100 \mathrm{GeV}$ (where the EBL attenuation is negligible given their redshift) is required in order to constrain better their intrinsic spectrum. The advantages of joint-spectral fits with GLAST and ground-based instruments to study the EBL attenuation of TeV blazars are discussed in section 5.5.2.

\subsubsection{GLAST Prospects}

As described in the last section, ground-based $\gamma$-ray telescopes have measured the attenuation of $\gamma$-ray sources by the near- and mid-infrared part of the EBL. Unfortunately, the strong opacity experienced by very high energy photons $(E>100 \mathrm{GeV})$ limits $\gtrsim 0.1 \mathrm{TeV}$ probes of the EBL to low redshifts, and thus, measurements such as those described above are useful to constrain the current level of EBL flux only, since they do not offer an insight into the evolution of the EBL.

GLAST, on the other hand, is sensitive to the less drastic attenuation of multiGeV photons by the UV-optical part of the EBL, with no attenuation expected (at any redshift) for photons with energy below $10 \mathrm{GeV}$. Thus, EBL attenuation will not limit GLAST's ability to detect blazars. Although the luminosity function of blazars 
at $\mathrm{GeV}$ energies is unknown (this is something that GLAST itself will measure), it is expected that GLAST's improved sensitivity with respect to previous missions will increase the number of known blazars to several thousands with redshifts up to z $\sim 3-5$. Because $\gamma$-ray sources to be observed by GLAST are distributed over a wide range of redshifts, EBL studies with GLAST will not only probe the total level of optical-UV background radiation, but its evolution as well.

The large expected number of GLAST blazars should allow for a fundamentally different approach to EBL studies, namely, the systematic study of EBL-induced signatures in the spectra of blazars as a function of redshift. This approach will help to address the question of whether the measured steepening in blazar spectra is due to intrinsic peculiarities in the sources, or the result of intergalactic absorption by the EBL.

The approach introduced in this dissertation is not the only way to study the EBL with GLAST observations of distant $\gamma$-ray sources. The effects of EBL absorption can also be measured by using emission models to predict the intrinsic spectrum of blazars through fitting of multi-wavelength data. Furthermore, blazars are not the only known class of extragalactic $\gamma$-ray sources. GRBs (gamma-ray bursts) experience the same type of attenuation and thus can be used to probe the EBL if they produce enough photons above $10 \mathrm{GeV}$ (which is not presently known and one of the important questions to be answered by GLAST). These two possibilities would constitute and independent type of analysis with respect to the one presented in Chapter 5, and when considered together, they will validate and complement each other.

The following chapter describes the GLAST instrument and its performance. The level of self-veto due to ACD backsplash of high energy photons (which are fundamental for EBL studies) is investigated in Chapter 4 with beam test data. Based on a detailed characterization of the instrument, two different methods to detect and measure the EBL attenuation of blazars are introduced in Chapter 5. 


\section{Chapter 3}

\section{The Gamma-ray Large Area Space Telescope (GLAST)}

\subsection{The Pair-Conversion Telescope Technique}

A pair-conversion telescope makes use of the pair-conversion interaction (described in sec. 1.1.1.2) to convert $\gamma$-rays into $\mathrm{e}^{+}-\mathrm{e}^{-}$pairs whose energy and direction can be measured as they pass through the detector system (see fig. 3.1). Momentum and energy conservation allow the pair to retain much of the information about the incident $\gamma$-ray, and thus, the direction and energy of the $\gamma$-ray can be reconstructed from the measured properties of the pair.

The design elements of a pair-conversion telescope are briefly outlined here by following the path of a $\gamma$-ray through a pair-conversion telescope. The details that are unique to GLAST are described in the next section.

The first detector system encountered by an incoming $\gamma$-ray is the anticoincidence detector (ACD, fig. 3.1). The primary use of an ACD is to detect the passage of charged particles that interact with the instrument. Space telescopes are exposed to a large flux of charged particles, which must be identified as background and eliminated from the data stream. 


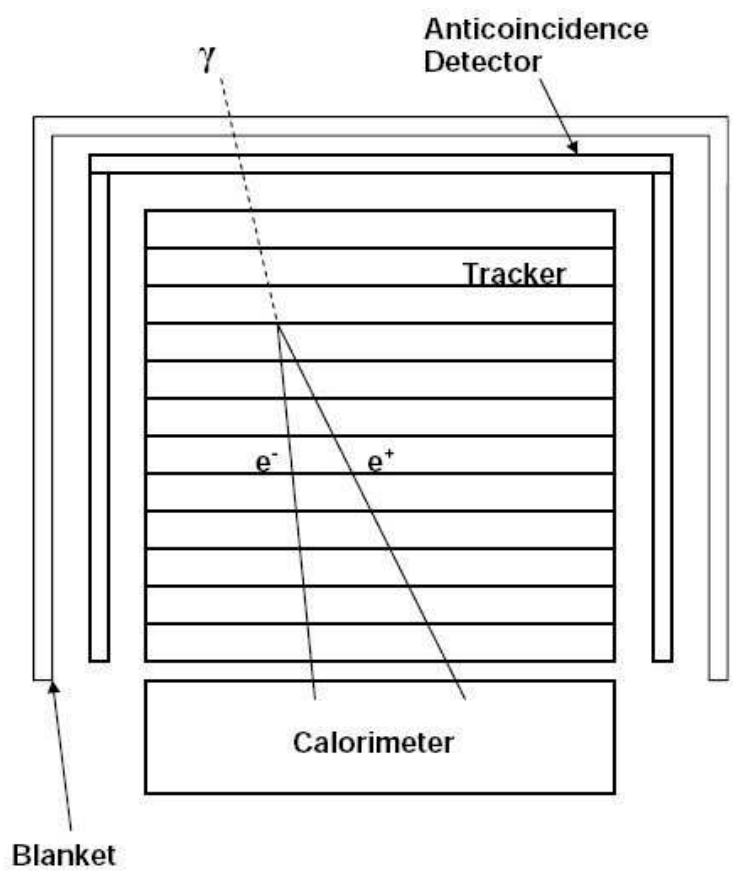

Figure 3.1: Diagram of a pair-conversion telescope, courtesy of [278]. The three active elements are shown: anti-coincidence detector, tracker and calorimeter. The blanket provides thermal insulation and shields against micro-meteoroids. A $\gamma$-ray enters the telescope leaving no signal in the anti-coincidence detector, then converts in a $e^{-}-e^{+}$ pair in the tracker, where the tracks are observed. Finally, the pair deposits their energy in the calorimeter. 
After passing through the ACD, a $\gamma$-ray enters the detector subsystem known as tracker (TKR). This detector consists of layers of high-Z material (usually Tungsten or Lead) which are placed between position sensitive charged particle detectors. The thickness and number of the high-Z material layers are chosen such that the total amount of material (measured in units of radiation length, section 1.1.1.2) induces the conversion of a $\gamma$-ray into a $\mathrm{e}^{-}-\mathrm{e}^{+}$pair with high probability. The pair is then tracked by the position sensitive particle detectors.

After crossing the TKR, the $\mathrm{e}^{-}-\mathrm{e}^{+}$pair (or any additional secondary particles) will enter the calorimeter (CAL) located at the base of the telescope (see fig. 3.1), which consists of scintillating material with good particle stopping power, such as Sodium Iodide (NaI) or Cesium Iodide (CsI). Because of the interaction mechanism of high energy particles in matter (sec. 1.1.1.2), the $\mathrm{e}^{-}-\mathrm{e}^{+}$pair (and any additional secondary particles) will produce an electromagnetic shower and deposit energy in the calorimeter. A measurement of the total energy can thus be obtained by measuring the response of the material (scintillation light from ionization), and if possible, by imaging the shower.

\subsection{GLAST LAT}

The Large Area Telescope (LAT) [188] builds upon the legacy of EGRET (sec. 1.1.3) and earlier pair-conversion telescopes [157]. The LAT was designed to avoid some of the limitations of EGRET (such as self-veto at high energies because of ACD backsplash, discussed in Chapter 4), and incorporates new technology and advanced on-board programmable electronics that will allow the LAT to achieve its scientific goals.

From the hardware point of view, the LAT is a modular pair-conversion telescope with a precision tracker and calorimeter (each consisting of a 4 x 4 array of 16 mod- 


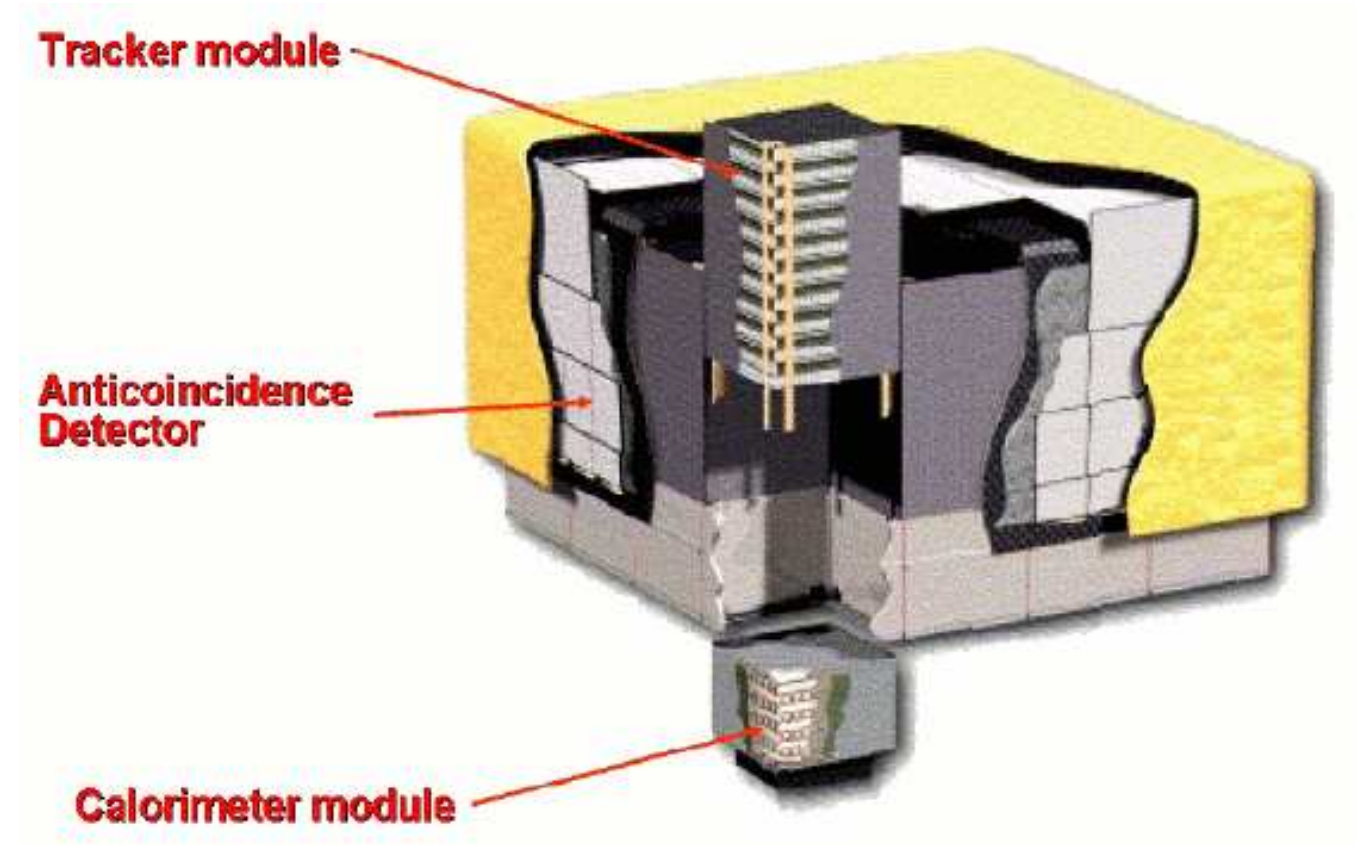

Figure 3.2: GLAST Large Area Telescope (LAT). The Anti-coincidence detector (ACD) is covered by the micrometeoroid shield and thermal blanket (yellow). A cutaway view of the TKR and CAL modules is also shown.

ules), a segmented anticoincidence shield that covers the tracker array (as illustrated in fig. 3.2), and a programmable trigger and data acquisition system. A detailed description of each one of the LAT components is presented below.

\subsubsection{LAT ACD}

\subsubsection{Design Considerations}

As explained above, the anti-coincidence detector (ACD) plays a crucial role in background rejection by detecting the passage of charged particles with very high efficiency [190]. This is the major driver of the ACD design.

Another very important driver of the ACD design is avoidance of the backsplash self-veto by high energy $\gamma$-rays. When the electromagnetic shower initiated by a $\gamma$-ray hits the massive calorimeter, many secondary particles are created (mostly 100-1000 
keV photons) [190]. Some of these secondary particles travel backwards and cross the ACD, where they can produce a signal via Compton scattering. These signals are usually associated with the passing of charged particles and could thus become responsible for the rejection of a perfectly valid $\gamma$-ray. EGRET, with its monolithic ACD dome was unable to mitigate this effect, which resulted in a significant loss of acceptance for $\gamma$-rays with energy above a few $\mathrm{GeV}$ [262].

The LAT avoids backsplash self-veto thanks to the segmentation of the ACD into "tiles" that are read out individually. The working principle is that an event is only vetoed if the trajectory of the incident particle (as reconstructed) can be extrapolated to a tile that has a measurable signal or "hit" (as would be the case for a charged-particle event). In the case of $\gamma$-rays, backsplash hits in the ACD tiles are not necessarily associated with the particle track and therefore the event is not vetoed. Of course, the possibility remains that a high-energy gamma will unfortunately produce backsplash hits in the ACD tiles associated with the particle track and the event will be rejected. Detailed Monte Carlo simulations show that by using the segmented ACD, as outlined here, the effects of backsplash self-veto can be mitigated (Monte Carlo simulations of the LAT are described in section 3.5.1). Chapter 4 focuses on the validation of this Monte Carlo simulations with a beam test of flight-like hardware.

\subsubsection{ACD Design}

The ACD consists of 89 tiles, arranged in the following way (see fig. 3.4):

- A top face $(+\mathrm{z}$ direction $)$ consisting of 25 tiles in a $5 \times 5$ array.

- Four side faces $( \pm \mathrm{x}, \pm \mathrm{y})$, consisting of 3 rows of 5 tiles each, plus a long tile at the bottom, for a total of 16 tiles per side.

The ACD tiles are made of E1Jen-200 plastic scintillator material, $10 \mathrm{~mm}$ thick (with the exception of the top central row tiles, which are $12 \mathrm{~mm}$ thick). After the passage 


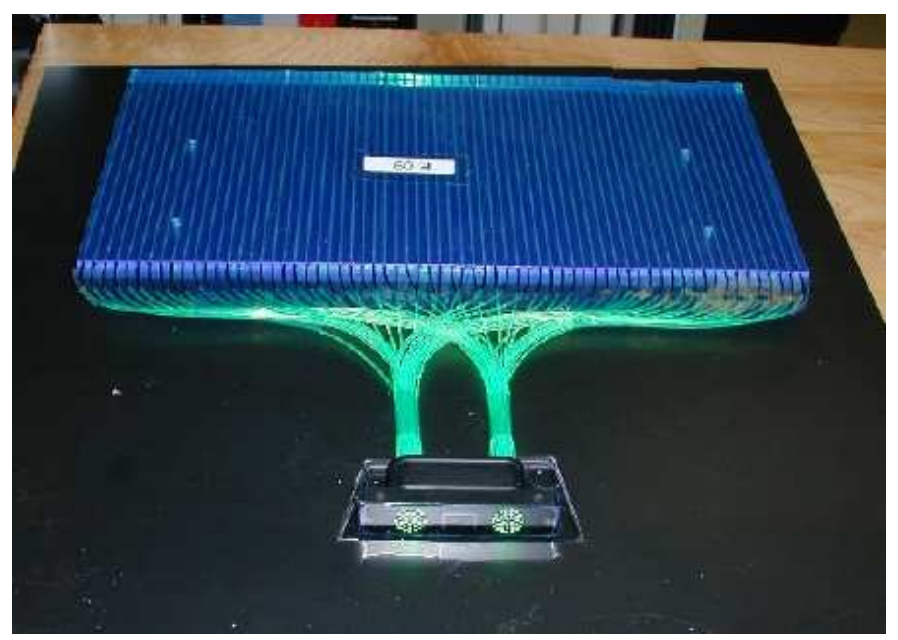

Figure 3.3: Tile with fibers (described in detail in [190]).

of a ionizing particle through the plastic, the scintillating light is collected by $1 \mathrm{~mm}$ diameter wavelength shifting fibers BCF-91A embedded in the plastic as can be seen in fig. 3.3. BCF-91A fibers were chosen because they have an absorption maximum at $425 \mathrm{~nm}$, precisely the wavelength at which the scintillating light from the plastic is maximum. As can be seen in fig. 3.3, the fibers within each tile are grouped into two different bundles. The light collected by each bundle is then delivered via fibers to different photo-multipliers (PMTs) for signal measurement. The splitting of the light output into two data streams is done for redundancy.

To simplify the assembly of the ACD and to reduce the amount of material within the field-of-view, it was decided to locate the PMTs at the base of the LAT, with distances to the scintillators exceeding one meter for the tiles at the top of the instrument. Since the scintillating light had to be transmitted over these distances, light attenuation became a significant issue. The wavelength shifting fibers used within the tile to collect the light are not well suited to transmit the light over long distances because of strong light loss (attenuation length ${ }^{1} \sim 4 \mathrm{~m}$ for fibers longer than 1 meter). The solution found was to couple the wavelength shifting fibers (coming out of the

\footnotetext{
${ }^{1}$ Light attenuation is expressed in terms of the attenuation length $\lambda$ : the fraction of light transmitted after a distance $\mathrm{L}$ is given by $e^{-L / \lambda}$.
} 

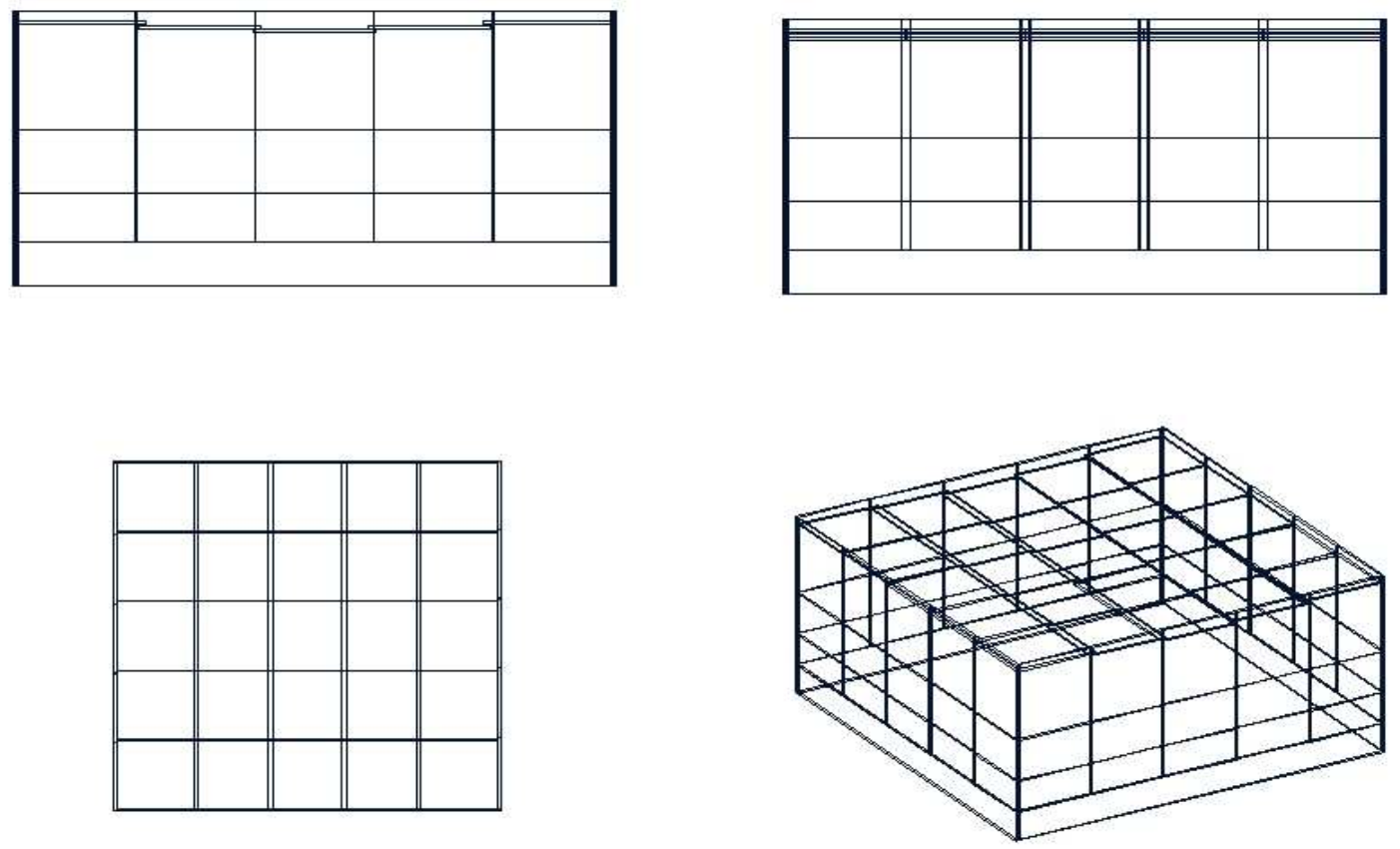

Figure 3.4: 4-views of the GLAST ACD tile layout. The top ACD is composed of 25 tiles in a $5 \times 5$ array. Each side consists of 3 rows of 5 tiles and a bottom row that is monolithic.

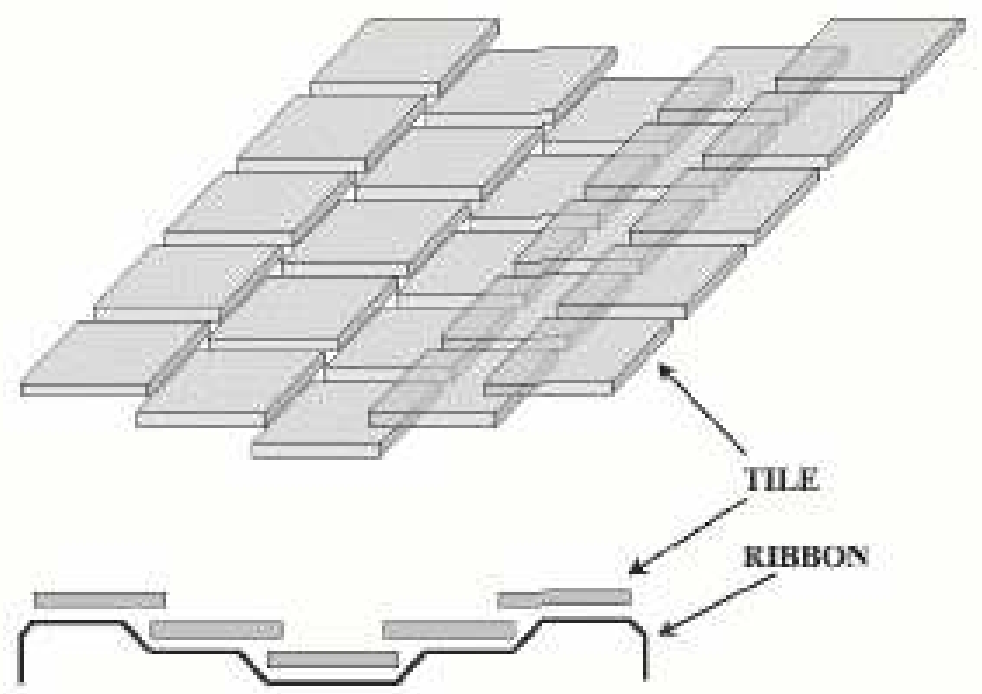

Figure 3.5: Schematic of top ACD tiles overlap (top) and its cross section (bottom), adapted from [190]. Each ACD ribbon covers the gap between two rows of tiles. 
tile) with clear fibers (attenuation length $\sim 6 \mathrm{~m}$ ) in those cases where the distance between a tile and one of its PMT was more than $50 \mathrm{~cm}$. In any other case, no clear fibers were needed.

Hamamatsu R4443 was the chosen PMT model because its high-gain (2 x $10^{6}$ @ $1250 \mathrm{~V}$ ) and low-noise maximize the sensitivity to low signals. The quantum efficiency of the PMTs was optimized upon request at $490 \mathrm{~nm}$, the peak wavelength for light transmission in the wavelength shifting fibers, with a resulting quantum efficiency that ranges between $16 \%$ and $23 \%$. Another desirable characteristic of the Hamamatsu R4443 is its small size, which was strongly constrained by the necessity to accommodate 194 PMTs in a limited volume.

One of the consequences of ACD segmentation is the unavoidable presence of gaps between the tiles. These gaps are of the order of 2-3 mm to allow for thermal expansion and to avoid tiles hitting each other during the strong vibrations experienced at launch. The tile layout avoids some gaps by overlapping the tiles in one dimension (as seen in fig. 3.5). Nevertheless, some significant gaps remain that if left unattended would bring the total ACD detection efficiency below the required performance $(>0.9997$ for single charged particle detection). The solution employed was to cover the gaps with flexible scintillating fibers or "ribbons". Each ribbon consisting of 25 separate fibers arranged in three layers, approximately 3 meters long. Each ribbon was carefully shaped to fit (as seen in fig. 3.5) the three-dimensional gap profile produced by the tile layout, with a total of 8 ribbons used to cover all gaps.

The ACD is surrounded by a MicroMeteoroid Shield (MMS) and thermal blanket designed to protect the instrument from high-speed micrometeoroids (that could impact and damage the instrument), and from temperature fluctuations in space. The MMS with a total area density of $0.39 \mathrm{~g} / \mathrm{cm}^{2}$ has a $95 \%$ probability of allowing no more than 1 penetration in five years according to the NASA ORDEM2000 orbital debris model [158]. Although a "dead" spot resulting from a single penetration would 
affect significantly the ACD efficiency, the track information could still be used to eliminate events entering from this region, resulting in a modest reduction of the LAT acceptance.

Careful consideration was given to the total amount of inert material outside the ACD (mostly MMS and thermal blanket) because it provides target material for interactions of $\gamma$-rays and cosmic rays. Gamma-rays could interact via pair-production outside the LAT, resulting in the loss of the photon. Cosmic rays, more importantly, could produce spurious $\gamma$-rays (through $\pi^{0}$ production/decay and positron annihilation), which are impossible to distinguish from "astrophysical" $\gamma$-rays for those cases where there is no associated signal in the $\mathrm{ACD}^{2}$. The goal was to keep the total density per unit area below $0.34 \mathrm{~g} / \mathrm{cm}^{2}$, but a larger value was required $\left(0.39 \mathrm{~g} / \mathrm{cm}^{2}\right)$ because of the larger area of the LAT (greater chance to be hit by a modest size micrometeor over the lifetime of the mission) and because of increases in orbital debris.

\subsubsection{ACD Electronics}

The ACD electronics reads out the signals from the 194 PMTs $^{3}$ with 12 circuit boards known as FREE (FRont End Electronics). Each FREE has capacity for 18 channels (for a total of 216), but just 15-17 channels in every board are used to service PMTs. Each board functions independently and communicates directly with the LAT central electronics (to be discussed later). For redundancy, the two signals from each tile (one signal for each fiber bundle) are always sent to PMTs in different boards, so that if one of the board fails, the tile (ribbon) can still be read with the other PMT.

Each FREE board consists of 18 analog Application Specific Integrated Circuits (ASICs) referred as GAFEs (GLAST-ACD Front End), 18 analog-to-digitalconverters (ADCs) and one digital ASIC referred as GARC (GLAST-ACD Readout

\footnotetext{
${ }^{2}$ These type of events constitute a source of irreducible background (sec. 3.5.2.3)

${ }^{3}$ (89 tiles +8 ribbons) x 2 PMTs per channel
} 


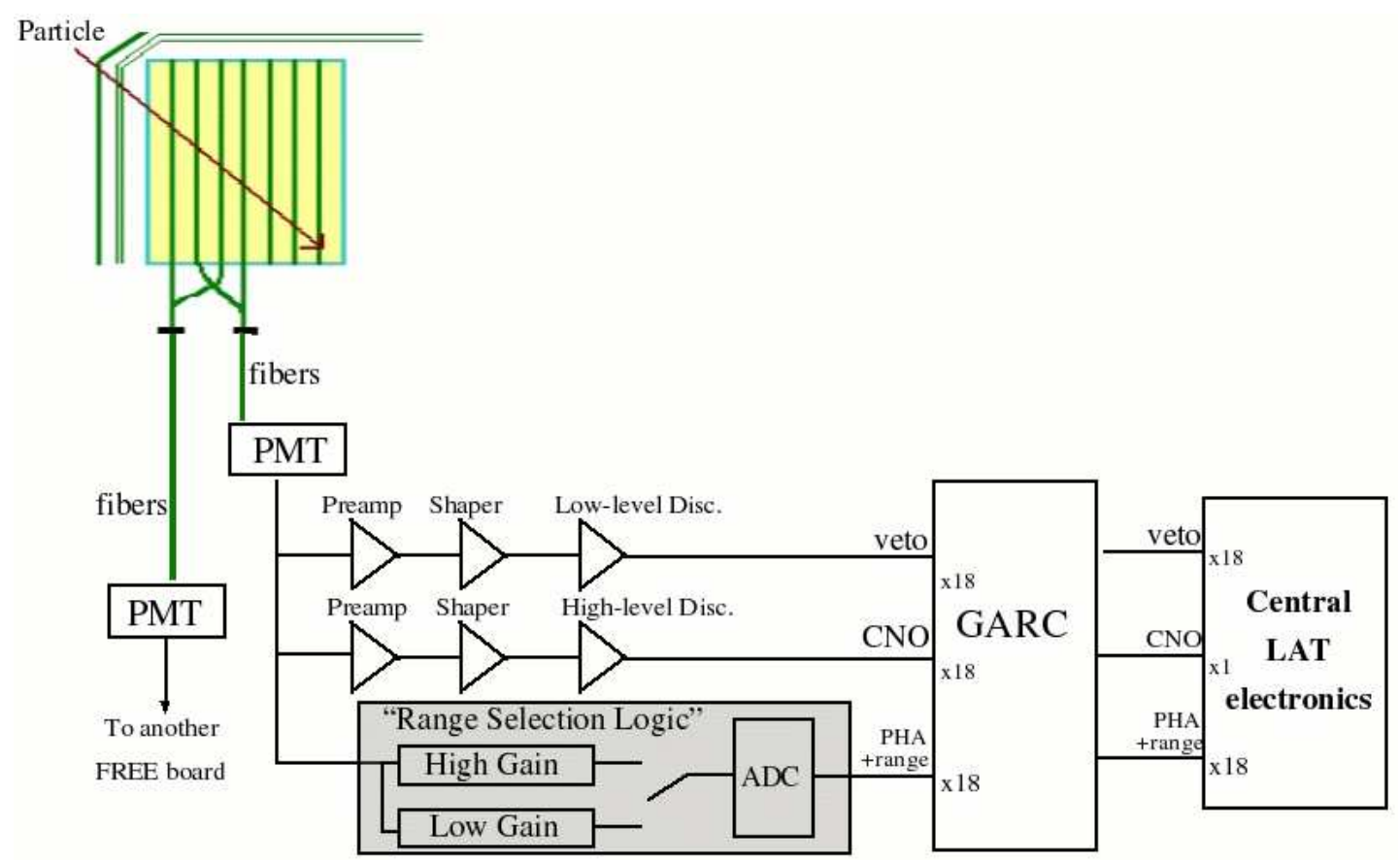

Figure 3.6: Schematic representation of ACD electronics.

Controller). The GAFE (one per channel) splits the analog signal from its PMT into two signals: a low-level discriminator (LLD) signal and a high-level discriminator (HLD) signal. The LLD (or veto signal) is discriminated against a low threshold $\left(0.45 \mathrm{mips}^{4}\right)$ to inform the passage of any charged particle. The HLD signal, with a threshold of 20 mips, is used instead to detect the passage of heavy ions (Carbon, Nitrogen, Oxygen, etc.) and is often referred as $C N O^{5}$ signal. Both signals are separately amplified, shaped and discriminated. The peak shaping time of both signals is equal to $3.2 \mu$ s and the discriminator threshold can bet set through a configuration register. The functionality of the ACD electronics is illustrated schematically in fig. 3.6 .

An additional discriminator in the GAFE is used to control the choice between two

\footnotetext{
${ }^{4} 1 \mathrm{mip}$ (in lower case) is defined as the most probable energy deposited by a single-charged minimum ionizing particle (MIP), when crossing an ACD tile (10 $\mathrm{mm}$ plastic scintillator) at normal incidence. $1 \mathrm{mip}$ is equal to $1.9 \mathrm{MeV}$.

${ }^{5} \mathrm{CNO}$ ions are quite useful for calibration purposes because its energy deposition is very well understood and easy to identify (one peak per ion in a histogram distribution).
} 
gains (low and high) for the pulse height digitization in the ADC. This discriminator performs a "range selection logic" to avoid range saturation by selecting low gain (high range) if the signal is high $(\sim>10$ mips), or high gain (low range) in the opposite case. This is the signal that is forwarded to the ADC for digitization ${ }^{6}$.

\section{Trigger Output}

Each GAFE produces veto (low-level discriminator) and CNO (high-level discriminator) signals. The 18 CNO signals in a free board are ORed by the GAFE so that only one CNO signal is transmitted to the Global Trigger Electronics Module (GEM), along with the individual veto signals from the 18 channels. The GEM receives then from the whole ACD system: 216 tile-like $^{7}$ signals (18 per free board) and 12 CNO signals (1 per free board)

\section{Event-readout Output}

When requested to perform event data readout by the central LAT electronics, the signal from each channel is digitized by the FREE. Depending on the signal strength, either low gain or high gain is applied to the signal before digitization. Thus for every channel (2 channels per tile), the ACD contributes the pulse height value (PHA) and the used gain (low or high).

\footnotetext{
${ }^{6}$ In order to avoid the digitization and handling of a large number of electronic noise (very low signals), a discriminator can be used to suppress the signals below a very low threshold. This is known as zero-suppression.

${ }^{7}$ Of the 18 channels available in each GAFE, a few (1-2) are empty while others correspond to ribbons. Ribbons are not designed to provide useful information during triggering, thus the signals coming from them are usually ignored.
} 


\subsubsection{LAT Tracker}

\subsubsection{Design Considerations}

The LAT performance depends on three quantities that are determined in great measure by the tracker design [261]: the effective area, the single-photon angular resolution, and the field of view. The effective area measures the probability that an incident photon will be detected and well reconstructed by the instrument, and therefore, depends on the amount of material in the conversion layers: with increasing total material thickness, the better the chance the photon will undergo pair-production. This is especially critical for the detection of high-energy photons which are scarce.

The single-photon angular resolution -better known in astrophysics as PointSpread Function (PSF)- is determined by the ability to reconstruct the direction of the incoming $\gamma$-ray from the instrument data. This in principle is limited by ratio of the detector resolution to the vertical lever arm over which the measurement can be made. Although a long lever arm will be highly desirable, this is strongly restricted in reality by the necessity to make the direction measurement before significant multiplescattering takes place in the subsequent conversion foils (at low energies) or the first bremsstrahlung photon has been emitted by the electron or positron (at high energies). This will be discussed in section 3.4.1. One of the features of the LAT design was the need to balance the trade-off between the use of thin foil converters (to minimize multiple-scattering and improve PSF at low energies), and thicker foils that increase the effective area. A hybrid design with thin and thick converters (described in next section) was chosen.

The field of view depends on the ability of the LAT to detect and reconstruct photons that are incident with large angles with respect to the axis of symmetry of the instrument $(+\hat{z})$. The TKR is shorter than it is wide, because such aspect-ratio ensures that the majority of the events will interact with the calorimeter. Unlike 
other instruments, the LAT does not require a time-of-flight system to distinguish upward from downward incident particles thanks in part to the use of a segmented calorimeter, which is used to image the shower and thus determine if the event is going up or down.

\subsubsection{TKR Design}

The LAT tracker [12] contains Tungsten converter layers and Silicon-strip detectors (SSDs). Tungsten layers are used to induce the conversion of a $\gamma$-ray into an electronpositron pair, while SSDs are used to track the trajectories of such particles. Each one of the 16 TKR modules is organized in a hierarchical structure: SSDs are combined into ladders, ladders are combined into planes, planes into layers and layers into towers. This hierarchical structure will be followed now to describe each one of the TKR components.

SSDs are a common type of solid-state detector. LAT SSDs (see fig. 3.7) consist of p-type strips implanted on a n-type silicon wafer $400 \mu \mathrm{m}$ thick. The strips are $56 \mu \mathrm{m}$ wide and have a separation or "pitch" of $228 \mu \mathrm{m}$, with 384 of them being implanted on each wafer. An aluminum backplane is bonded to the n-type material, while the p-type strips are covered with aluminum strips, but separated from them by a thin insulator. When a charged particle passes through the detector, the ntype material is ionized by an amount that is proportional to the particle charge and momentum, as described by the Bethe-Block formula [19]. The free electrons created by ionization drift towards the positivity biased (100V) aluminum plane, while the "holes" left behind drift towards the p-type strip and induce a charge on their corresponding aluminum strips [12]. The charge of the strip is measured by converting the current into a voltage, so that the passage and energy loss of the charged particle can be determined from the signal. Because of the large number of electron/hole pairs created by the passage of a charged particle ( 32000 electron/hole 
pairs for a minimum ionizing particle at normal incidence), the signal-to-noise ratio is very high, and this translates into a very high hit efficiency.

Another key advantage of SSD technology is that the separation between strips can be made very small, so that the passage of a charged particle can be pinpointed with high resolution. Hence, the passage of a charged particle results in a collection of "hits" across the TKR layers that clearly show the trajectory or "track" of the incident particle. SSD technology is also advantageous because of self-triggering capability, low dead-time $(<10 \mu \mathrm{s})$, and absence of consumables.

Four SSDs (and their individual strips) are bonded in series, with the strips connected by aluminum wire bonds, to form a 4-SSD long ladder as seen in fig. 3.8. Then, four ladders are placed next to each other in a 4x4 SSD array to form a Silicon plane. The resulting plane contains $1536(384 \times 4)$ strips or channels and is about 35 cm x 35 cm large.

When a charged particle passes through a plane, it will produce an electric signal in one, or maybe a few, nearby strips. From the location of the hit strips it will possible to calculate the point of particle crossing along the base of the plane. It should be noted however, that it is not possible to know where along the strip the particle went through. In this respect, the orientation of the strips determines which position measurement $(x$ or $y$ ) is obtained from a single plane. By using two planes whose strips are orthogonal to each other, the $(\mathrm{x}, \mathrm{y})$ position of the crossing point can be measured. The combination of $\mathrm{x}-\mathrm{y}$ planes is referred to as a layer.

Detector planes, converter foils and readout electronics are packed into trays for a practical and convenient mechanical design. Each tray is about $3 \mathrm{~cm}$ thick and contains a structural panel for support that is made of aluminum honeycomb with carbon composite faces and four carbon-carbon ${ }^{8}$ lateral closeouts (fig. 3.9). The

\footnotetext{
${ }^{8}$ Carbon-carbon composites consist of a carbon matrix reinforced with carbon fibers. These materials are often used because of their structural strength and thermal stability.
} 


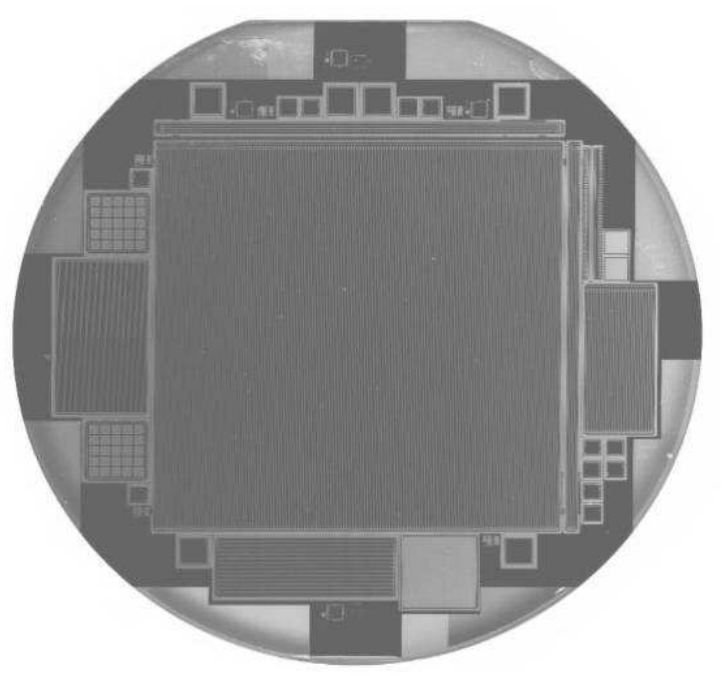

Figure 3.7: Picture of a SSD (seen as a square shape at the center) before being cut out (described in detail in $[232,33]$ ). Each SSD contains 384 strips (aligned vertically in the picture) with a $0.228 \mathrm{~mm}$ pitch.
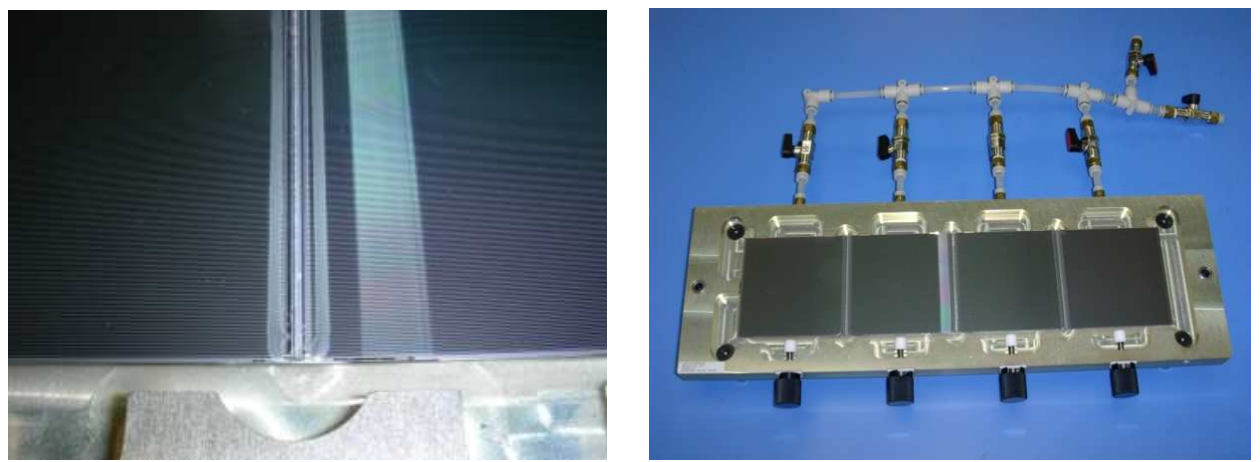

Figure 3.8: TKR ladder: a) Close-up of the strips bond, and b) full 4-SSD ladder in a test-bed (described in detail in $[232,33]$ ). 
Tungsten foil is attached under the structural panel and two planes of silicon with the same orientation ( $x$ or $y$ ) are placed at the top and bottom of the tray, as indicated in fig. 3.9. The readout electronics consists of two Multi-Chip electronics Modules (MDMs) that are mounted on two opposite sides of the tray in order to minimize the dead area.

Since particle tracking requires determination of $x$ and $y$ positions of the hits, $x$ and y planes are placed next to each other. This is achieved by alternating trays that are rotated $90^{\circ}$ with respect to each other. Two consecutive trays form therefore a $x-y$ set of planes separated by a $2 \mathrm{~mm}$ gap (even though they are packed in different trays). The TKR design has the converter foil right above the $x-y$ pair of layers, ensuring that the electron and positron directions are measured immediately after the conversion, which is critical for the PSF.

A total of 19 trays are stacked on top of each other to form a TKR module that can be seen in fig. 3.10. The trays at the top and bottom of the stack only contain one silicon plane (since in those cases only one $x$-y layer can be formed with the only tray that is adjacent). This results in $18 x-y$ layers with a $3 \mathrm{~cm}$ separation between them.

In order to balance the need for thin converters (good PSF at low energy) versus the need for large amount of converter material (larger effective area), each TKR module contains two sections, "front" (or thin) and "back" (or thick). The front section consists of the top 12 trays (and 12 associated x-y tracking layers) which were provided with thin foil converters, each $0.12 \mathrm{~mm}$ thick (for 0.03 radiations length each). The back section meanwhile, has converter foils $0.72 \mathrm{~mm}$ thick (for 0.18 radiation length each) on trays 13 through 16 (4 x-y tracking planes). The 3 bottom trays (2 x-y tracking planes) have no converter foil present because a photon converting in such place could only produce hits in two x-y layers, which as will be explained later, would not trigger the instrument. 


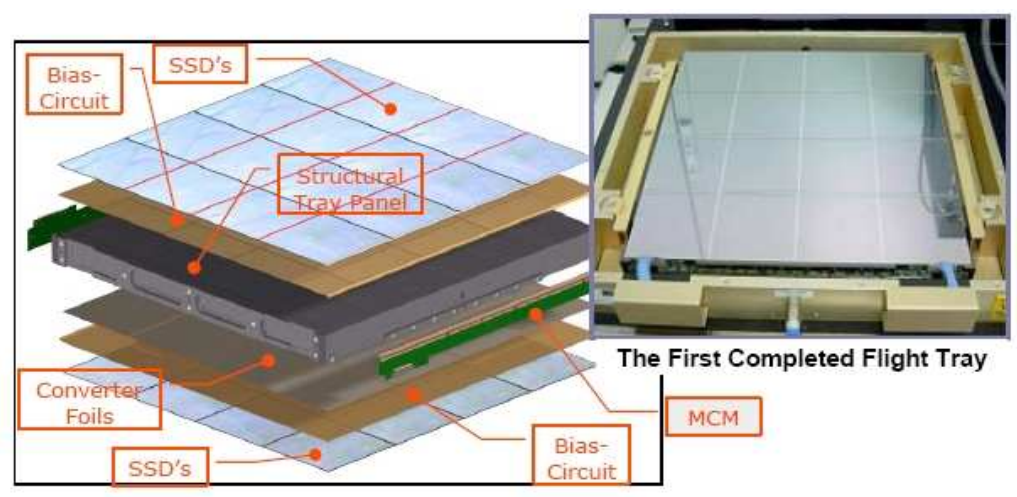

Figure 3.9: [Schematic representation of a tracker tray] Tracker tray consisting of two silicon panels, converter foil and structural panel (described in detail in [261]).

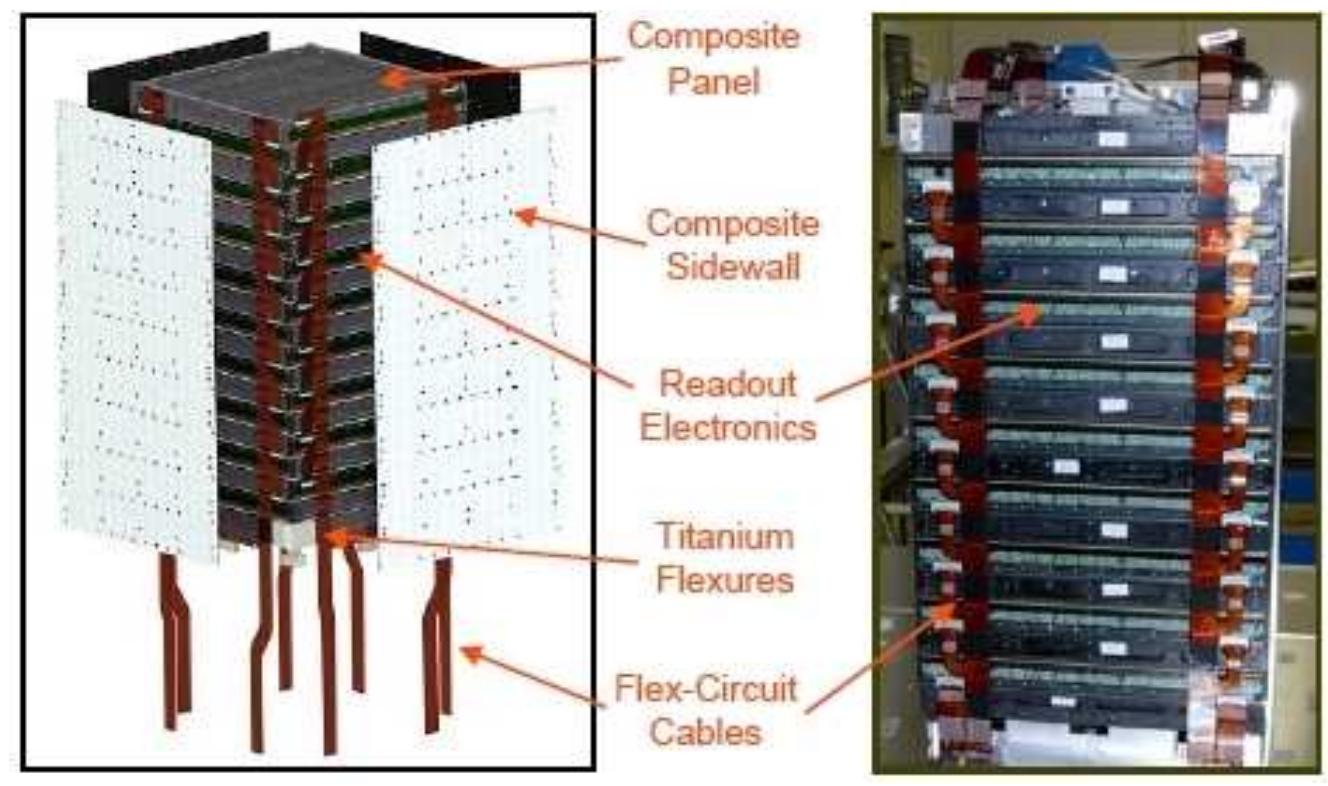

Figure 3.10: TKR module (described in detail in [261]): schematic (left) and picture of inverted module with sidewall removed (right). 
The stack of 19 trays is contained and supported by carbon-composite sidewalls which also serve to conduct heat to the TKR base. The TKR module is mechanically assembled to a $4 \times 4$ grid through eight titanium flexures located at the base (see fig. $3.10)$.

\subsubsection{TKR Electronics}

In each tray the readout electronics are mounted on the lateral walls. The 1536 channels in each layer are divided into 24 groups (64 channels each) that are read by custom-designed ASIC chips referred as GTFEs (GLAST Tracker Front End). Each one of the 24 GTFEs amplifies and shapes the signal from 64 different strips. No attempt was made to digitize the pulse height of every strip, instead a comparator is used in every channel to discriminate the signal against a common programmable threshold. The only output from a single channel is therefore a binary signal that indicates if the pulse is over threshold. Nevertheless, pulse-height information is available through the time-over-threshold measurement, which is described in the event-readout section below.

The 24 GTFEs reading out a given plane are serviced by a separate custom digital chip, the GLAST Tracker Readout Controller (GTRC). For redundancy, every plane uses two GTRCs. Each GTRC nominally services half of the GTFEs in a layer, but the split point is arbitrary and if desired, a single GTRC could service all 24 GTFEs. The GTRCs are located on opposite ends of the MCM (tray side) and it is common then to refer to the left and right ends of a plane.

Each GTRC communicates with the TEM (tower electronics module; [119]) through one of eight flex-circuit cables. Each cable is responsible for a plane-end, which implies that every cable services 9 plane-ends. If required (as in the case of a cable failure), the whole plane can be read through the cable servicing the other end, providing redundancy to the system. 
Calibration mask and capacitors

Trigger and Data mask registers

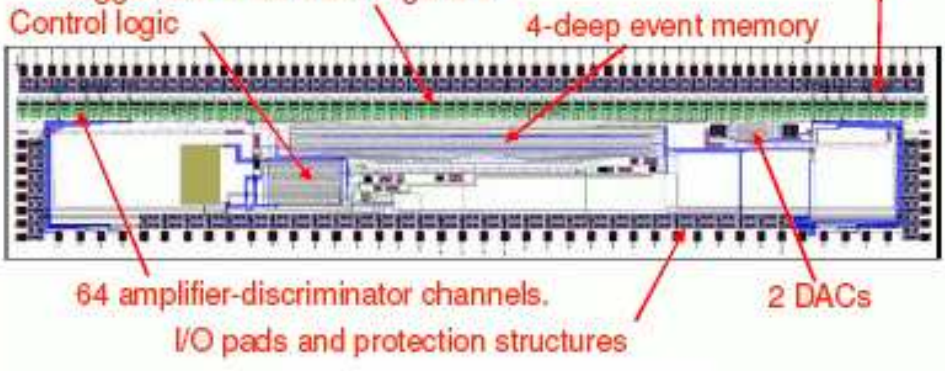

Amplifier-Discriminator Chip

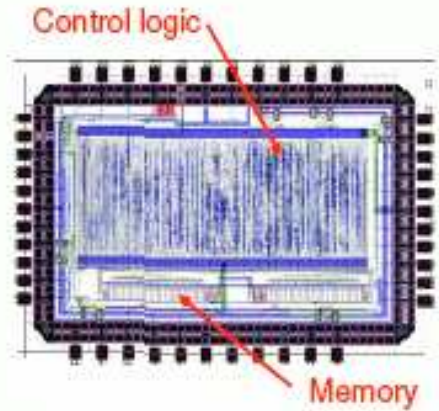

Readout Controller Chip

Figure 3.11: GTFE (left) and GTRC(right) chips (described in detail in [203] and [209] respectively).

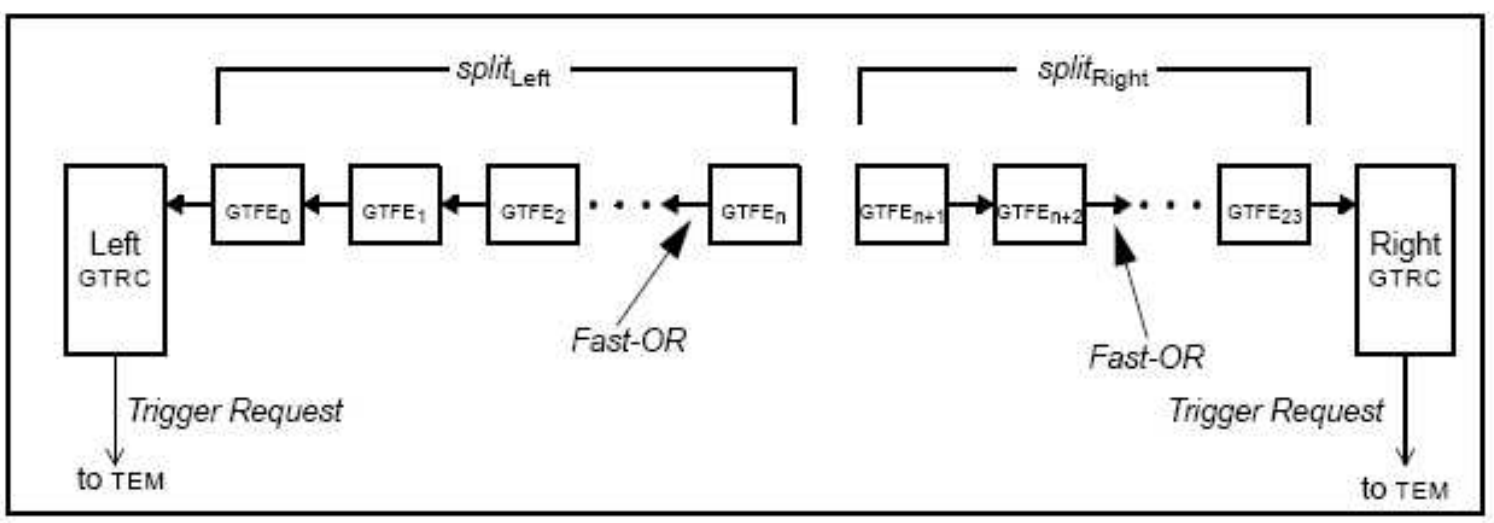

Figure 3.12: Fast-OR signal from GTFEs, from [115]. 


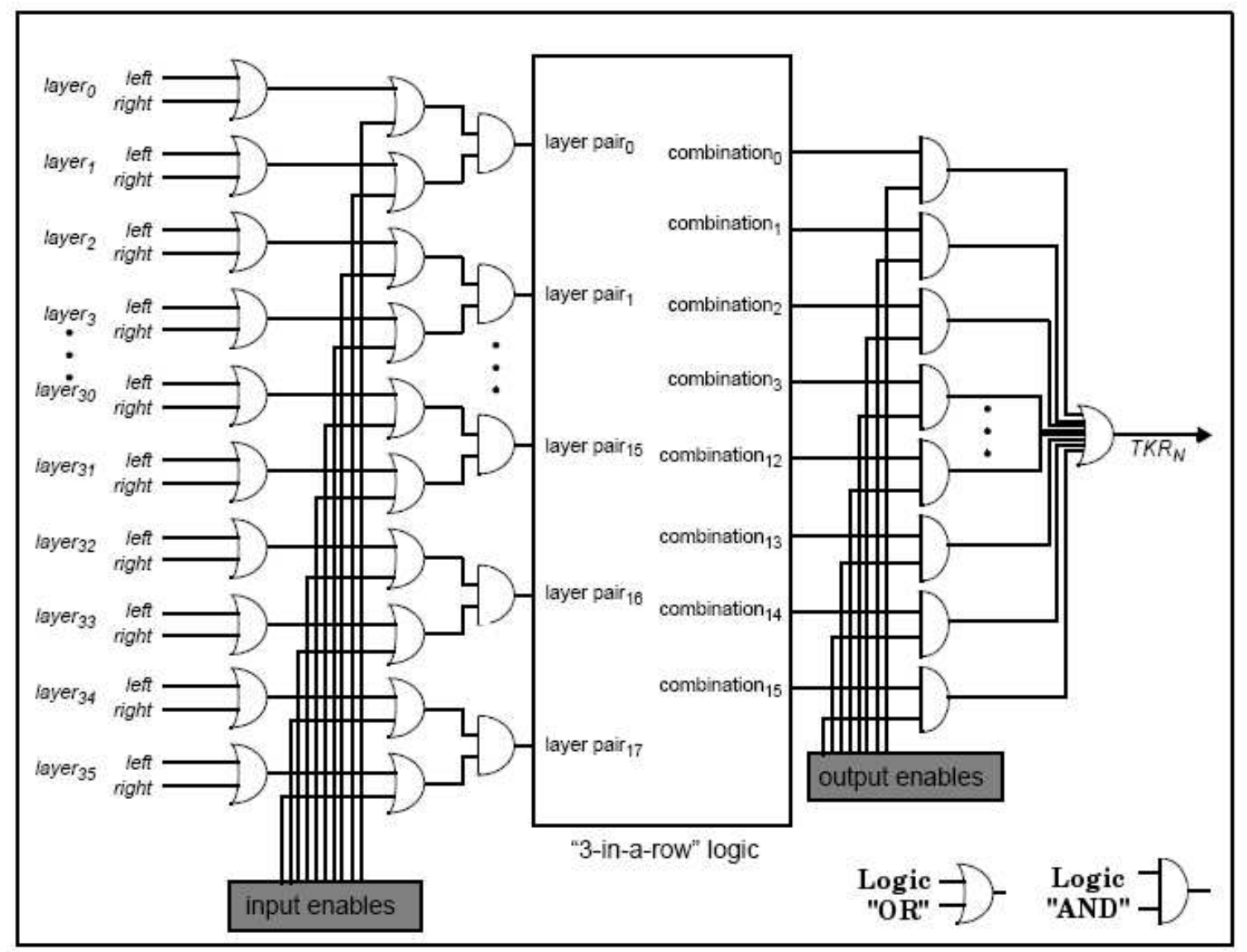

Figure 3.13: TKR trigger logic diagram, adapted from [115]. The left and right layer signals are ORed together for form a single signal per layer. The 3-in-a-row algorithm looks then for the coincidence of $3 \mathrm{x}$-y layer pairs, with 16 possible combinations (from 18 layer pairs). Finally, these 16 combinations are masked and ORed together in order to issue a single TKR trigger request per tower.

\section{Trigger Output}

Within each GTFE the 64 digital signals are masked individually and then ORed together to produce a single trigger related output signal called FAST-OR. The FAST$O R$ signals from every GTFE are chained together to form a single FAST-OR signal per plane-end, as indicated in fig. 3.12. The FAST-OR is processed by the GTRC of that end and then sent to the TEM as a trigger input. Thus, every TKR module contributes 72 trigger signals to the TEM, one for each plane-end.

Once in the TEM, the left and right trigger signals from each plane are ORed 
together into 36 plane signals as indicated in fig. 3.13. These 36 plane signals are the input for the 3-in-a-row algorithm. This algorithm looks for the coincidence of three adjacent $x$ - $y$ layer pairs in order to produce a TKR-trigger-request. The 3-in-a-row requirement is very effective to detect particle tracks while keeping the probability of noise-induced trigger quite negligible, since it requires a six-fold coincidence. The 3in-a-row algorithm calculates the logic value of the 16 possible combinations of layers which are then masked with an output enable register and ORed together to produce a single TKR trigger signal per tower. The trigger request from every tower is then sent to the GEM as a trigger primitive.

\section{Event-readout Output}

The TKR electronics also gathers the strip information that is going to be used later to reconstruct the event. The data of individual strips is passed from GTFE to GTFE (along with the FAST-OR) until it arrives at the GTRC. Once at the GTRC, a list is formed of the addresses of the channels with a hit. If a decision is made to trigger and keep the event, this information will be sent to the central LAT electronics system.

Another important piece of information provided by the TKR is the Time Over Threshold (TOT) of the FAST-OR signal from every layer-end, which is digitized by the GTRC. The TOT is linear with the charge deposited in the layer all the way up to 50-60 fC (equivalent to the charge deposited by $\sim 6$ mips at normal incidence [35]). Because the charge is proportional to the ionization, as described by the Bethe-Block formula [19], the TOT value substitutes the pulse-height value information (which is not digitized). For instance, a slow moving proton will experience increasing energy loss, and deposit increasing charge, as it passes through planes of silicon. In contrast, a MIP will deposit a much smaller and constant amount of energy in each layer. 


\subsubsection{LAT Calorimeter}

\subsubsection{Design Considerations}

The LAT calorimeter (CAL) makes use of CsI crystals to measure the energy and shape of the electromagnetic shower produced by a $\gamma$-ray. Apart from good energy resolution, one of the key requirements of the CAL is to provide precise determination of the energy centroid ${ }^{9}$ of the electromagnetic shower. This information is extremely important for event reconstruction since it provides an anchor point to the track finding algorithms. This is especially crucial for high energy events which produce a broad electromagnetic shower and multiple secondary tracks.

The CAL energy deposition pattern produced by an electron (or photon) is quite different from those produced by protons, muons or other particles. Since the physics of passage of electromagnetic particles through matter is well known, the profile of the shower measured by the segmented calorimeter can be used to differentiate $\gamma$ ray events from other particles. Furthermore, the depth of the shower maximum for electrons increases with the log of the energy; a fact that can be used to distinguish particles going downwards from particle going upwards (which are background).

For very high-energy events, the electromagnetic shower will not be completely contained in the CAL, and a significant fraction will leak through the side faces or bottom of the calorimeter. This will result in a raw energy deposition that is not equal to the total event energy. Leakage corrections are then applied to the measured energy by fitting the contained part of the shower to a known shower profile curve. Another type of correction applied to the measured energy takes into account the gaps and dead material in the calorimeter region, which was minimized as much as possible during the design of the sub-system (less than $16 \%$ of the total mass).

\footnotetext{
${ }^{9}$ Energy-weighted mean geometrical center of the energy deposition.
} 


\subsubsection{CAL Design}

The calorimeter is built as a $4 \mathrm{x} 4$ array of identical modules that match one-by-one the TKR modules. Each module contains 96 CsI crystals with dimensions $2.67 \mathrm{~cm} \mathrm{x} 1.99$ cm x $32.6 \mathrm{~cm}$ arranged in 8 horizontal layers of 12 crystals each, as shown in fig. 3.15. CsI is a well known scintillator with a very good stopping power, fast response and adequate light yield. Each log is optically bonded at each end to two photodiodes: i) the large diode with an area of $1.5 \mathrm{~cm}^{2}$, which covers the range $2 \mathrm{MeV}-1.1 \mathrm{GeV}$, and ii) the small diode with an area of $0.25 \mathrm{~cm}^{2}$ that covers energy deposits up to $70 \mathrm{GeV}$. Crystals in even layers (also known as x-layers) are orthogonal to crystals in odd layers (y-layers), thus forming a $x$-y hodoscopic array [128]. The full calorimeter's depth is 8.5 radiation lengths for normal incident particles (10 radiation lengths total when a considering the TKR also).

The size of each crystal was chosen to balance the need for large light yield (at least 5000 electrons per $\mathrm{MeV}$ for the large diode and 1100 electrons per $\mathrm{MeV}$ for the small diode ${ }^{10}$ ), and good spatial resolution (given by the location of the log and therefore limited by its size). It should be noted that in addition to the two discrete spatial coordinates provided by the location of the crystal in the stack, the light output asymmetry of the photodiodes at each end is used to calculate via a simple analytic function the location of the energy deposition along the crystal. The threedimensional location information together with the energy deposition reported from each crystal are used then to calculate the energy centroid of the event.

The mechanical design of the calorimeter minimized the dead material in the instrument while safely providing support for the heavy array of crystals $(\sim 80 \mathrm{Kg}$ per module) that during launch can be accelerated up to $\sim 6 \mathrm{~g}$. The material used for support must also have a low atomic number in order to minimize the chance of

\footnotetext{
${ }^{10}$ As measured when a MIP crosses within $\pm 3 \mathrm{~cm}$ of the crystal center
} 


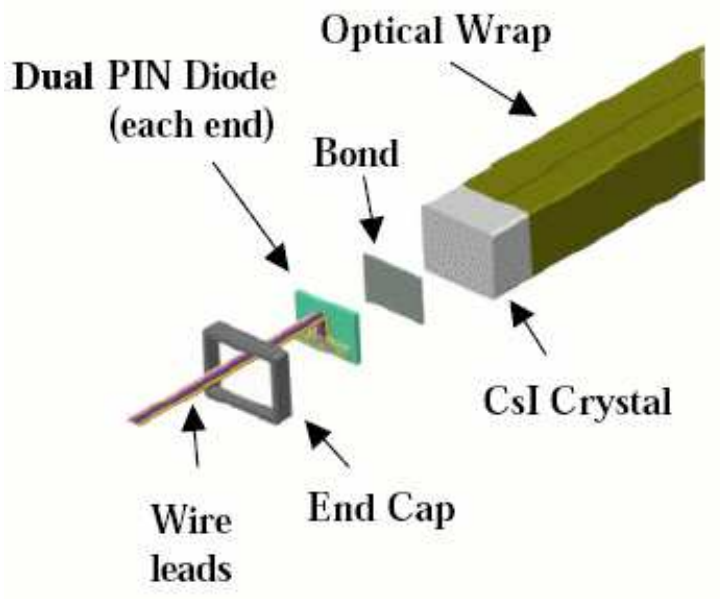

Figure 3.14: GLAST calorimeter crystal log (described in detail in [147]).

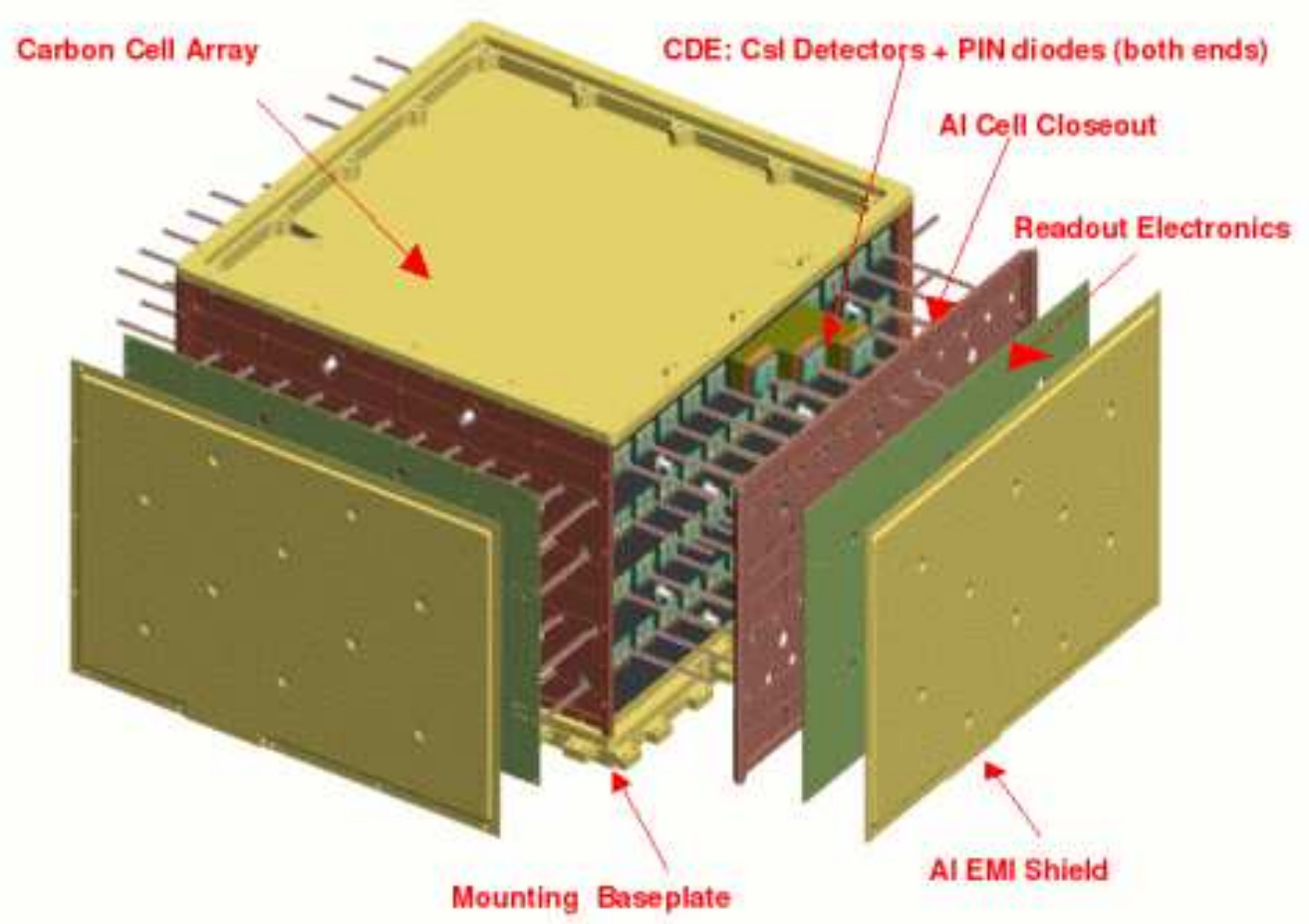

Figure 3.15: GLAST CAL module (described in detail in [30]). The carbon fiber composite structure, CsI detectors, readout electronics boards and Aluminum ElectroMagnetic Interference (EMI) shields are shown. 


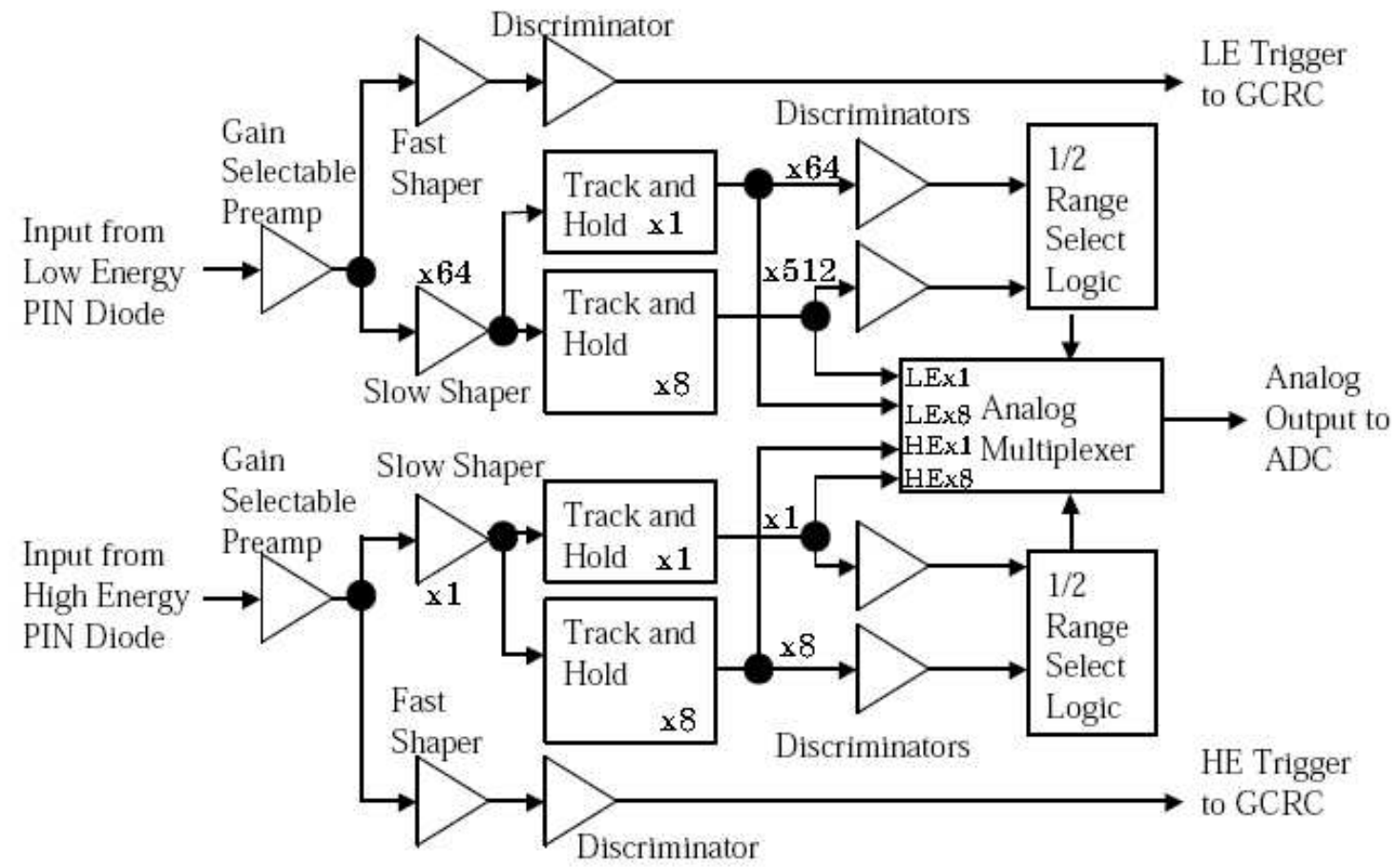

Figure 3.16: GLAST Calorimeter Front End (GCFE) functional block diagram, adapted from [115]. The signals from each diode are used for trigger primitives (fast shaping) and data readout (slow shaping). The data readout has 4 different gain ranges ( 2 per diode). The multiplexer sends the signal from one of the ranges to the ADC for digitization (unless 4-range readout is requested, in which case the signal from the four ranges are digitized). Not shown: DACs used for calibration of the GCFEs, calibration injection and digital logic circuits.

interactions with the electromagnetic shower from an event. The basic mechanical concept chosen was therefore a carbon fiber composite box which is shown in fig. 3.15 . The readout electronics are mounted on four electronic boards, known as Analog Front End Electronics (AFEE), that are located on the sides of the CAL module between the aluminum cell closeout and the outer Electro-Magnetic Interference (EMI) shield. Each board services 48 crystal ends (4 layers x 12 crystals).

\subsubsection{CAL Electronics}

The two photodiodes from every crystal end are connected via a cable lead to an ASIC chip known as GLAST Calorimeter Front End (GCFE), for a total of 48 GCFEs per 
board [115]. Each GCFE is responsible for both fast response signals that are used as trigger primitives, and readout of event data that will be used to reconstruct the event (described below). The functional block diagram of a GCFE is shown in fig. 3.16. The digitization of each channel is performed by a dedicated ADC.

The GCFE and ADC signals from each one of the 12 crystal ends that constitute a CAL-module-row are sent to a digital GLAST Calorimeter Readout Controller (GCRC) chip in the AFEE (4 GCRC per board). The GCRC serves as an interface between the single-crystal-end dedicated electronics and the Tower Electronics Module (TEM) as illustrated in fig. 3.17. In general terms, each GCRC performs functions of control, data readout, data packing, and housekeeping for its associated GCFE and ADC chips.

\section{Trigger Output}

The GCFE takes the output from the small (high energy) and the large (low energy) photodiodes and passes each one through a preamplifier and a fast shaper $(\sim 3.5 \mu \mathrm{s})$. If the signal is above an adjustable threshold (that can be set individually for every channel), a CAL trigger request of the type CAL-low (set nominally at $100 \mathrm{MeV}$ ), or CAL-high (set nominally at $1 \mathrm{GeV}$ ), or both, will be issued and forwarded to the GCRC.

The CAL-low and CAL-high trigger outputs from every GCFE (one per crystal end) are ORed together (OR) into a single $C A L$-low and $C A L$-high signal per CAL layer as indicated in fig. 3.18. The GCRC receives these signals and forwards them to the TEM. Each TEM receives therefore $C A L$-low and $C A L$-high trigger signals from 16 GCRCs (4 per face), which are ORed together to form one single CAL-low and CAL-high trigger signal per tower. Both signals are then sent to the GEM. 
CALORIMETER FUNCTIONAL BLOCK DIAGRAM

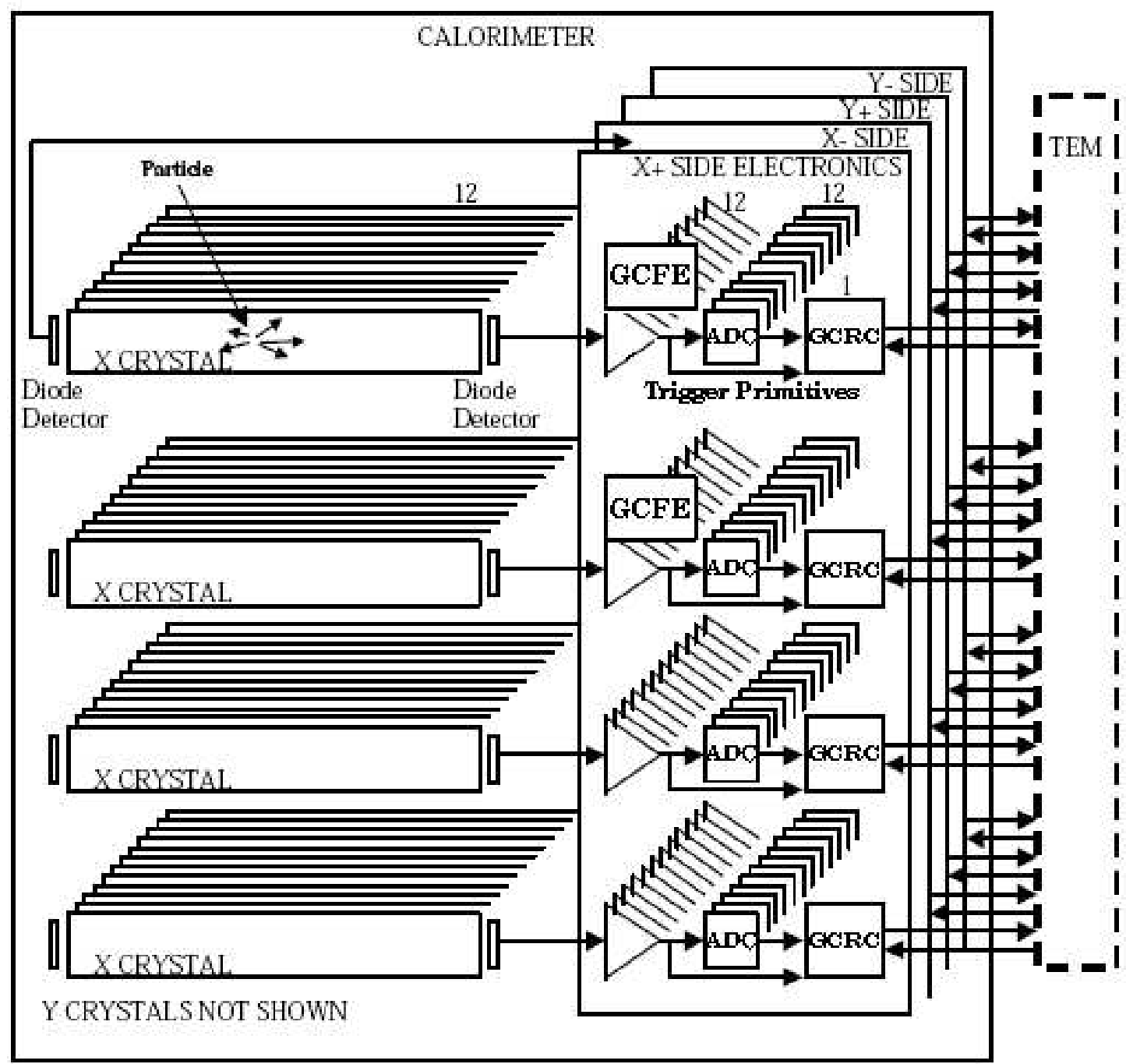

Figure 3.17: CAL electronics functional block diagram, adapted from [115]. Each GCFE sends trigger primitives (CAL_LO and CAL_HI) and event readout (through an ADC) to the GCRC. The GCRC serves as an interface between the TEM and the GCFEs and ADCs. 


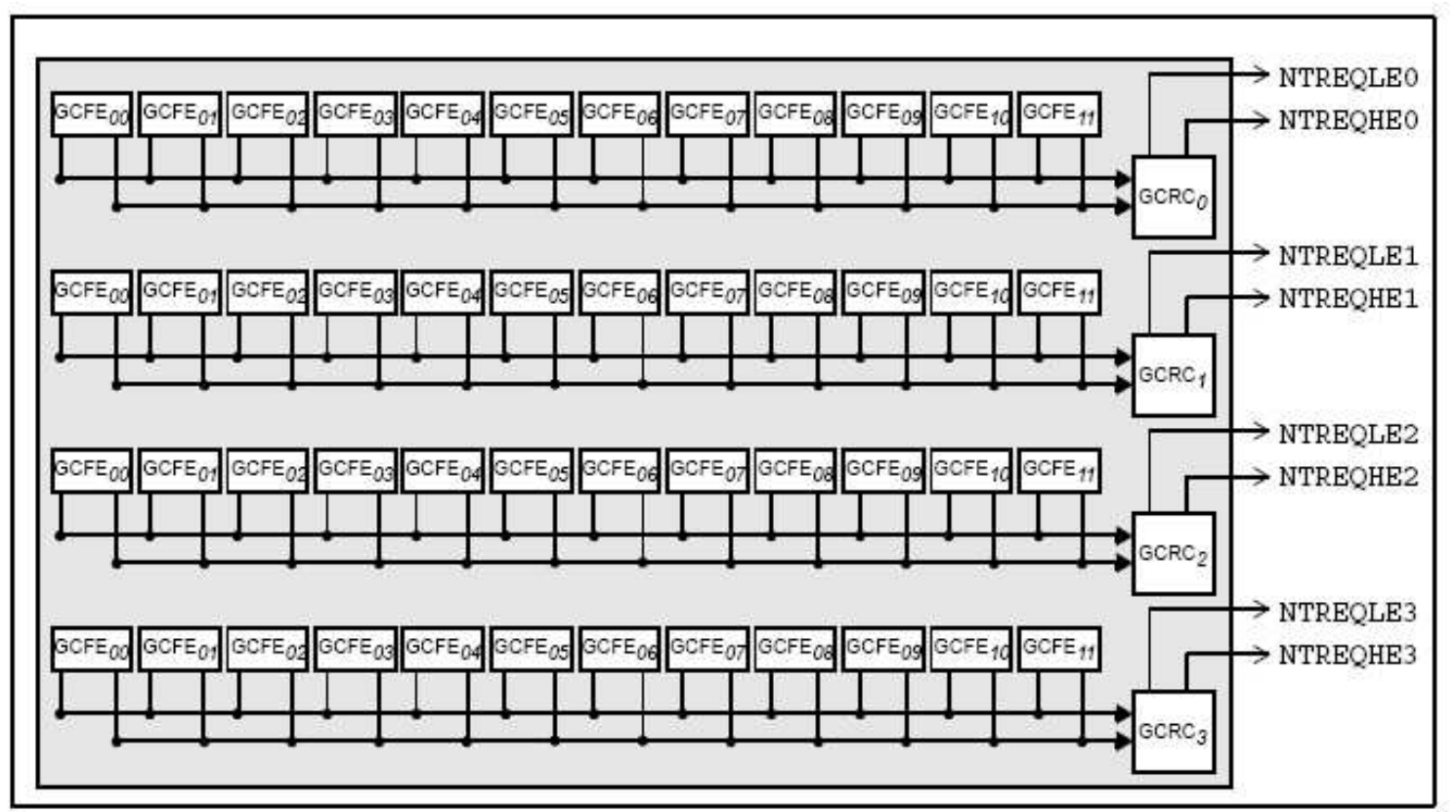

Figure 3.18: CAL trigger signals from a single AFEE, from [115]. The low- and high-energy trigger outputs from each GCFE are ORed by layers and delivered to the GCRC, and then forwarded to the TEM. A total of four circuit-boards like the one represented here process the trigger requests in every CAL module.

\section{Event-readout Output}

Each GCFE shapes and amplifies the signals from both diodes with a low and high gain $(\mathrm{x} 1, \mathrm{x} 8)$, for a total of 4 gain ranges per crystal end (with ratio 1:8:64:512) ${ }^{11}$. These different gains are needed because of the large dynamic range that each crystal is designed to cover $(\sim 2 \mathrm{MeV}$ to $\sim 70 \mathrm{GeV})$. When requested to perform event data readout by the central LAT electronics, the GCFE perform a "range selection logic" that selects the lowest unsaturated energy range, and nominally, this is the signal that is forwarded -via the analog multiplexer- to the 12-bit ADC for digitization. Nevertheless, the GCFE can be told by the LAT central electronics to use a specific gain, or for calibration purposes, the LAT can be set to 4-range readout mode, in which case all the gains are digitized sequentially. Obviously, the readout in this mode takes longer and the deadtime of the instrument increases. To decrease the

\footnotetext{
${ }^{11}$ The measured signal ratio is somewhat different than the nominal one (1:9:60:540).
} 
data volume, it is possible to suppress the transfer of the digitized data for signals below the Log Accept (LAC) threshold, which can be set individually for each crystal end (useful if the channel becomes noisy). In its default mode of operation, the CAL contributes for every channel (2 channels per crystal): i) pulse height value (PHA), and ii) a two-bit word that indicates the gain used. Of all the subsystems, digitization of the CAL signals is the most time consuming, and therefore, it sets the deadtime of the instrument $(\sim 27 \mu$ s per event $)$.

\subsubsection{LAT Data Acquisition (DAQ) System}

The Data Acquisition system (DAQ) makes all the detector sub-systems work as a coherent unit. It takes care of triggering the instrument, sending the data to the onboard filter for background rejection, and packing the event into an event structure for data transmission to the ground.

\subsubsection{Global Trigger}

The LAT trigger system takes signals from each subsystem (TKR, CAL, ACD) as input and determines if an event is to be recorded. Before making a trigger decision, the trigger system reduces the trigger signals from the ACD in the following way:

The two veto signals from each tile are consolidated (ORed) into a single signal per tile. This results in 108 tile inputs that are sent to the Region of Interest (ROI) generator for further reduction. A ROI is a user-defined subset of the 108 tiles $^{12}$ of the ACD, which may include as few as none or as many as all 108 tiles. The tile subsets from different ROIs are allowed to overlap without any restriction. However, the trigger system supports a maximum a 16 ROIs. The ROIs are useful during

\footnotetext{
${ }^{12}$ There are only 89 physical tiles in the ACD. However this description is taking place in "electronics space" where it doesn't matter if the electronic channel is coupled to a PMT or not. In practice, the electronic channels without a physical tile attached to them don't produce any output and can be safely ignored.
} 
trigger formation because they offer a flexible way to interpret the ACD-veto data. Indeed, the ROI signals can be used i) to trigger the instrument, or more often ii) to veto a trigger decision (hardware trigger veto, defined below).

In addition to the ROIs, the following signals are also considered inputs (primitives) for the trigger:

- TKR 3-in-a-row (from all the TKR modules)

- CAL-Low (from all CAL modules)

- CAL-Low (from all CAL modules)

- CNO (from the GAFEs. Each GAFE contributes the consolidated CNO signal from 12 tiles)

- Periodic (a fixed rate signal from the system clock)

- Solicited (received from the control unit)

The fact that any number of trigger primitives is affirmative does not imply that such trigger request would be acknowledged. Indeed, one of the most important functions of the hardware trigger is to look for patterns in the trigger primitives that suggest that the signals are caused by a cosmic ray and not a $\gamma$-ray. In such occasions, the trigger system prevents the instrument from triggering and reading out the event. This functionality is known as hardware trigger veto. The hardware trigger is flexible to allow optimization of trigger efficiency (once more information about the background is obtained on-orbit), and is also versatile to accommodate for various event signatures [120]. The advantage of the hardware trigger veto is that it rejects background particles before event read-out, thus reducing the time the instrument is busy because of background events.

In particular, the hardware trigger veto makes use of the shadowing of towers by ACD tiles to reject charged particles. Each tower in the LAT has a configurable list 
of ACD tiles that shadow it, and this set is conveniently used to define a ROI (one per tower). When a cosmic ray passes through the LAT, it will create an ACD veto signal and a TKR trigger request (3-in-a-row) in at least one of the towers. Because of ROI shadowing, the ACD tile that went over threshold belongs to the ROI associated with the triggered tower (or one of the triggered towers, if more than one), and thus, the hardware trigger veto can make use of the coincidence of ROI and TKR signals (associated by shadowing) to avoid triggering on events that are very likely due to charged particle background. It should be noted however, that the lower two rows on the side faces of the ACD are not used in the ROI definition to avoid removing two types of event: i) $\gamma$-rays whose conversion products scatter out the sides, and ii) $\gamma$-ray events with ACD backsplash ${ }^{13}$.

The hardware trigger veto also makes use of the CAL trigger information. Indeed, the hardware trigger will not reject any event that has a CAL-low or CAL-high primitive, independently of the ROI + TKR situation. This is done to ensure that no significant fraction of gammas is rejected by the hardware veto. Although this will allow background events to trigger the instrument, the purpose of the hardware veto is to reduce the trigger rate, not to get rid of all background events. More detailed background rejection algorithms are performed at later stages.

The hardware trigger is implemented through a configurable register that indicates the system what trigger decision to take when any combination of the trigger primitives is met. In this context, the hardware trigger is global because it considers all possible trigger inputs from all subsystems before making a decision. If the combination of trigger primitives results is an affirmative trigger decision and the instrument is not busy (reading out a previous event), the central electronics module issues a Trigger Accept Message (TAM) that is broadcasted to the subsystems. The

\footnotetext{
${ }^{13}$ Lower ACD tiles are closer to the CAL and thus are more likely than the rest to go over threshold because of backsplash radiation originated when a high-energy event hits the CAL.
} 
function of this message is to alert its recipients that their respective detectors must be read out, and also indicates how to read out (zero suppressed, 4-range readout, etc.).

\subsubsection{Onboard Filter (OBF)}

The space environment where the LAT will operate presents high fluxes of cosmic rays. Even with hardware trigger veto this background flux will trigger the instrument at a high average rate $(\sim 2.5 \mathrm{kHz})$, with an expected maximum of $\sim 6 \mathrm{kHz}^{14}$. With an average event size of 3 Kbits after compression, an average data transmission rate of 11 Mbits per second (Mbps) would be required to downlink the data to the ground. This represents a problem because the LAT's bandwidth only allows a maximum average transmission rate of $1.2 \mathrm{Mbps}$, i.e. a maximum event rate of $400 \mathrm{~Hz}$.

The Onboard filter (OBF) reduces the event rate to a value that meets the downlink bandwith requirement by rejecting enough of the events that have a clear background signature (see [278] for a complete description). Since the rate of astrophysical $\gamma$-ray events is expected to be a few $\mathrm{Hz}$ on average, the onboard filter is not required to reject background events with very high efficiency, indeed, this could have the dreadful consequence of rejecting a significant fraction of $\gamma$-rays. A more detailed background rejection can be done on the ground with far superior computing power and analysis tools. The OBF thus aims to reduce the background event rate only by a factor of $\sim 10$.

Events filtered out by the onboard software are those that meet a set of tests that search for background-like patterns in the combined signal output from all subsystems. The tests (or vetoes) can be grouped as follows (see [278] for a detailed description):

- Vetoes that look at the number and spatial distribution of hit ACD tiles. These

\footnotetext{
${ }^{14}$ Average and maximum background rates are not completely known. The LAT collaboration uses a set of background flux models that constitute the best estimates (see [211] for a detailed description)
} 


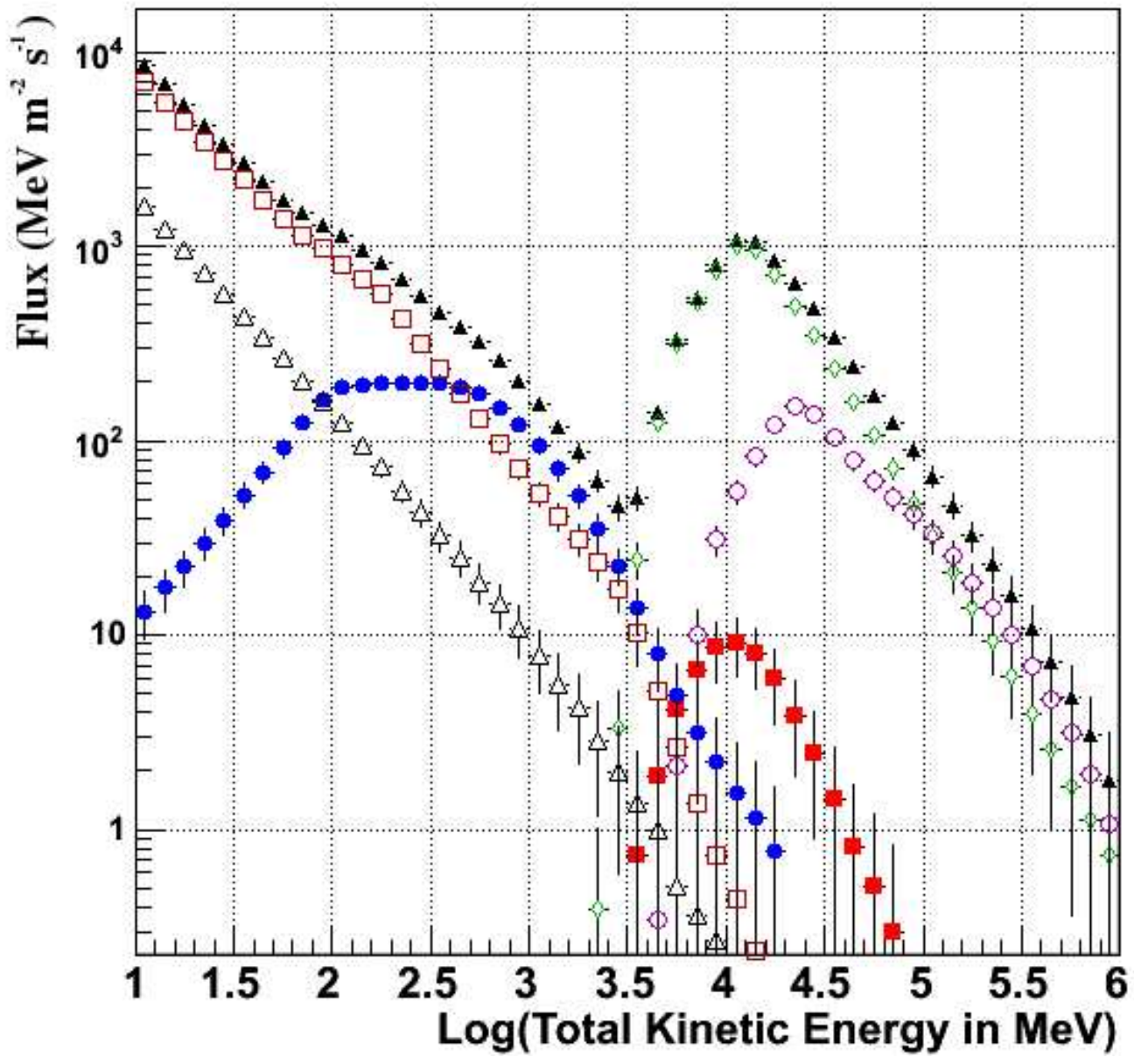

Figure 3.19: Expected average background flux as function of kinetic energy. The curves are as follows: total flux (black solid triangles), galactic cosmic ray proton flux (green empty diamonds), He + CNO flux (purple empty circles), galactic cosmic ray $\mathrm{e}^{-} \mathrm{e}^{+}$flux (red solid squares), albedo proton and antiproton flux (blue solid circles), electron and positron albedo flux (brown empty squares), and albedo $\gamma$-ray flux (black empty triangles). 
vetoes are generally disengaged by significant CAL energy depositions to avoid backsplash self-veto

- Vetoes that look for the association of hit ACD tiles with TKR hits. These vetoes are similar to the hardware trigger veto but are not restricted to towers with a TKR trigger (3-in-a-row) (towers with non-consecutive layer hits are considered)

- Vetoes that look for events without enough TKR hits for track reconstruction, since such type of events have limited scientific value

- Vetoes that look for particles moving upwards in the CAL based on the energy deposited in each CAL layer

- Vetoes that use a rudimentary track-finding algorithm to veto events whose $\operatorname{track}(\mathrm{s})$ points to hit $\mathrm{ACD}$ tiles, or to the gap region between the top of the CAL and the ACD.

The vetoes are evaluated sequentially. When the on-board filter determines that an event has failed a veto, it notes the reason for the veto in a summary word and stops processing the event. This ensures that no computing power or time is wasted. A pass-through mode can be enabled to allow vetoed events to continue into the data stream, at a prescaled rate, for diagnostic purposes.

One of the most important design characteristics of the on-board filter is its flexibility: individual vetoes can be turned on and off, energy thresholds and other selection parameters can be tuned, etc. Indeed, the on-board filter configuration will be evaluated and tuned during the first year, as more information is gathered by the LAT about the background fluxes and their effects on the LAT trigger ${ }^{15}$.

\footnotetext{
${ }^{15}$ To monitor on-orbit backgrounds and study filter performance, the on-board filter will send to the ground and unbiased sample of all trigger types at an average rate of $\sim 30 \mathrm{~Hz}[120]$.
} 
On a final note, the filtering capabilities of the onboard software are scientifically important, even in the hypothetical case of unlimited bandwidth. The LAT is expected to detect Gamma-ray Bursts (GRBs) on its own, but this is only possible if the onboard detection algorithms can be run with a reasonable clean sample of $\gamma$-rays [278]. Otherwise, the transient signal from (even bright) GRBs will be swamped by the enormous amount of background events.

\subsection{LAT Simulation}

The main tool for study of the instrument performance is the Monte Carlo simulation software. The software contains a extremely detailed description of the LAT geometry that includes the instrument components, their location, constituent materials and noise levels. The simulation makes use of a widely used high-energy physics code known as GEANT4 [91] to model the particle propagation through the instrument (pair conversion, bremsstrahlung, multiple-scattering, etc.) and the response of the detectors (energy depositions in silicon strips, CAL crystals and ACD tiles). The software also includes a simulation of the hardware trigger and an embedded copy of the on-board filter [278]. The output of this simulation (digital signals from the instrument detectors) is delivered to the event reconstruction software in the same format that real data would have.

Gamma-ray and background fluxes are provided to the Monte Carlo simulation by a fully configurable source engine that can emulate a wide range of astrophysical and calibration sources. A detailed study of simulated data has been used to characterize the instrument response functions that are described in section 3.6.1.

Obviously, Monte Carlo simulations are useful as long as they correctly represent the real instrument. Thus, thorough verification of the simulation has been central to the design, construction and review phases of the instrument. The accuracy of 
the simulation has been tested by placing actual detector hardware in beams of high energy particles (photons, electrons, protons, etc.) whose energy and direction are known. Several beam tests have taken place:

- Beam test in 1997 to verify technology choices and Monte Carlo simulations $[15]$

- Beam test in 1999-2000 of ACD tiles, TKR and CAL detectors at SLAC with beam energies up to $30 \mathrm{GeV}$ [61]. This beam test focused on sub-systems integration, data acquisition performance, and validation of Monte Carlo simulations for on-axis and off-axis incident beams, including hadron beams.

- Beam test in 2006 of the LAT Calibration Unit (actual flight hardware) at CERN with beam energies up to $280 \mathrm{GeV}$. Results from this beam test will be discussed in Chapter 4, where an analysis of ACD backsplash will be presented.

\subsection{Event Reconstruction}

The raw information contained in each event includes:

- ACD: PHA value and gain range for every PMT (2 PMTs per tile)

- TKR: strips with a hit (out of 884736 total strips) with time over threshold (TOT) information from every plane-end

- CAL: PHA value and gain range for every crystal end

- LAT electronics: trigger condition summary, trigger primitives, timestamp, housekeeping data, and so forth.

The event reconstruction software takes this data as input and produces tracks (direction, energy), energy measurement (total raw energy, corrected energy, energy 


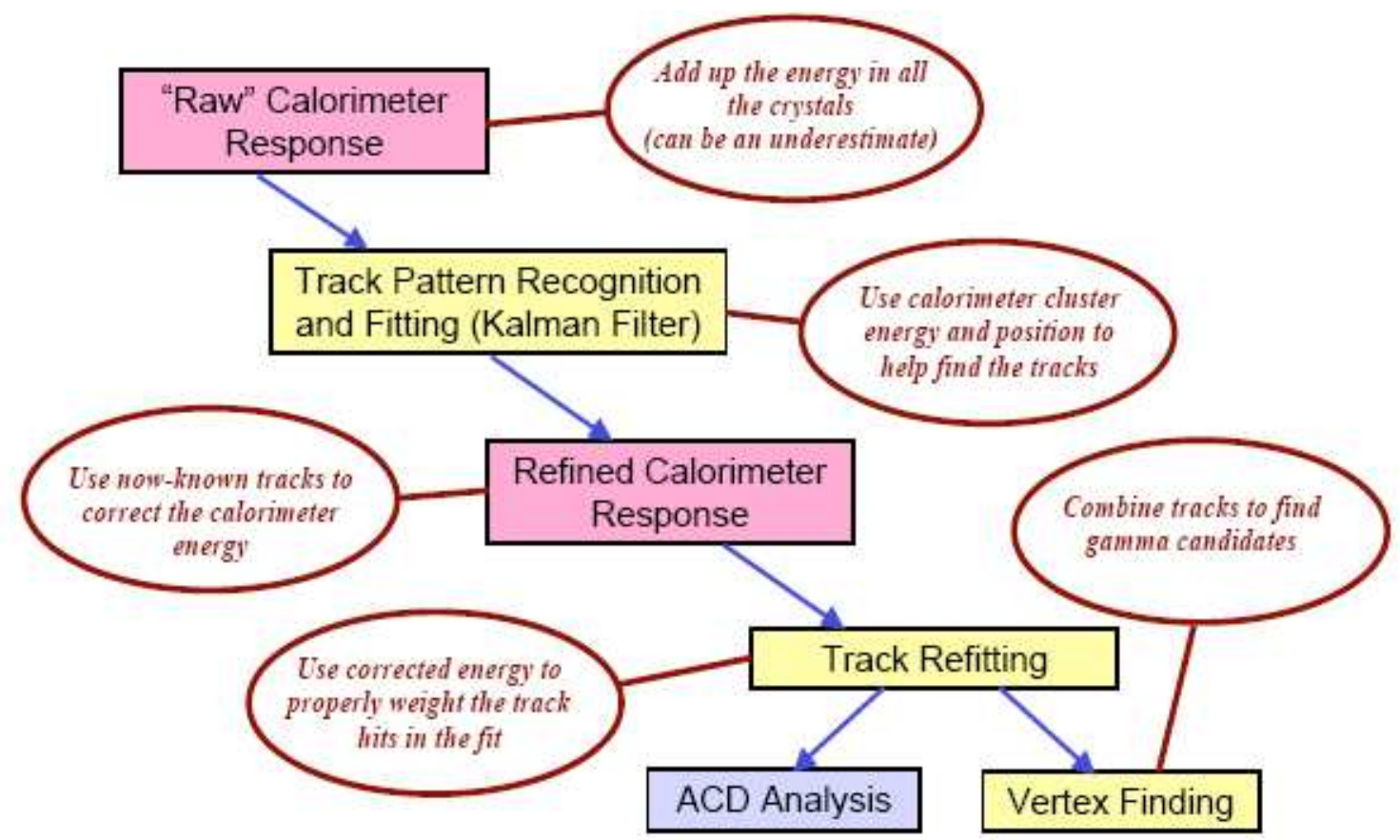

Figure 3.20: Schematic representation of LAT event reconstruction. From [231].

centroid) and related information (TOT, tracks intersecting ACD tiles, MIP tracks in the CAL, and so forth). The steps involved in event reconstruction are described below (see fig. 3.20 for a schematic representation).

\section{Raw Calorimeter Response}

The first step in energy reconstruction is the conversion of the PHA value from every crystal end to deposited energy (taking into account the associated gain range). The energy from each end is i) summed to find the total energy in the crystal, and ii) compared in order to find the point along the log where more energy was deposited (light asymmetry). The information from all logs is then used to obtain: i) the total CAL raw energy, ii) the energy-weighted centroid, and iii) a three-dimensional image of the shower (thanks to the hodoscopic design). 


\section{Track Pattern Recognition and Fitting}

Reconstruction of the particle tracks begins by grouping nearby strip hits into clusters. Starting with clusters in the uppermost layer that trigger, a line is drawn to the CAL energy centroid (if no centroid is present, clusters in layers below are used). This line guides the algorithm to find clusters in the layer right below that could be associated with the track. If no clusters are found close to the line, the next layer is searched. By stepping down the layers and associating clusters with the line, a possible track is formed. However, multiple clusters could be present in a given layer, and sometimes the algorithm ends up with a large list of possible tracks. For every track in the list a quality parameter is calculated from:

- Number of hits

- The $\chi^{2}$ value obtained by fitting to a straight line

- How high the track started (tracks that start high are more likely to be associated with the original $\mathrm{e}-\mathrm{e}^{+}$pair)

- Missing layer hits

Up to this point, the track reconstruction has been based on pattern recognition algorithms that decide which hits are part of a track, with no physics involved. The challenge now consists of making the best estimate of the track caused by a physical particle (electron, muon, proton, etc.) in order to find its incoming direction and $\operatorname{energy}^{16}$.

\footnotetext{
${ }^{16}$ The energy estimate from the TKR is not nearly as good as the energy determination from the $\mathrm{CAL}$, but nevertheless, it is useful information for background rejection. Furthermore, low-energy particles will lose a significant fraction of their energy in the TKR, requiring then to add CAL and TKR estimates for the total energy determination.
} 
The LAT event reconstruction makes used of an implementation by Frühwirth [88] of the Kalman filter [132] that is widely used in experimental particle physics. At low energies, the track propagation is dominated by multiple-scattering and the best the filter can do is to connect the dots. The incoming $\gamma$-ray direction is then calculated geometrically from the two upper hits, while the level of multiple-scattering is used to calculate the energy according to the relation [74]

$$
\theta_{\mathrm{rms}} \simeq \frac{0.015 \mathrm{GeV}}{p} \sqrt{z / X_{0}}
$$

where $\theta_{\text {rms }}$ is the root mean square of multiple scattering, $p$ is the particle momentum and $z / X_{0}$ is the material thickness in units of radiation lengths.

At high energies, multiple-scattering is negligible and the error is dominated by the hit position (error measurement). The best track is obtained then with a leastsquared-fit to a straight line of the TKR hits. In the intermediate energy band, the filter provides an optimal balance for the competing effects of multiple scattering and measurement error. The Kalman filter provides a logically consistent, mathematically rigorous basis to find the best track in all energy regimes.

The filter consists of two consecutive processes: the filter and the smoother [127]. The filter begins at the first (uppermost) hit of a track and makes a prediction of the location of the next hit according to its current guess of the track parameters (direction and energy) at that point. The prediction is then refined in light of the measured hit location (track parameters are recalculated and error matrices are updated). This iterative process continues to the end of the track (see fig. 3.21). When the filtering is finished, the track estimation at any given point does not have any feedback from the hits below. Smoothing takes care of this by step-backing up the track, refining the track parameters at each point based on the information down the track (as illustrated in fig. 3.22). After the second step is completed, a reliable determination 


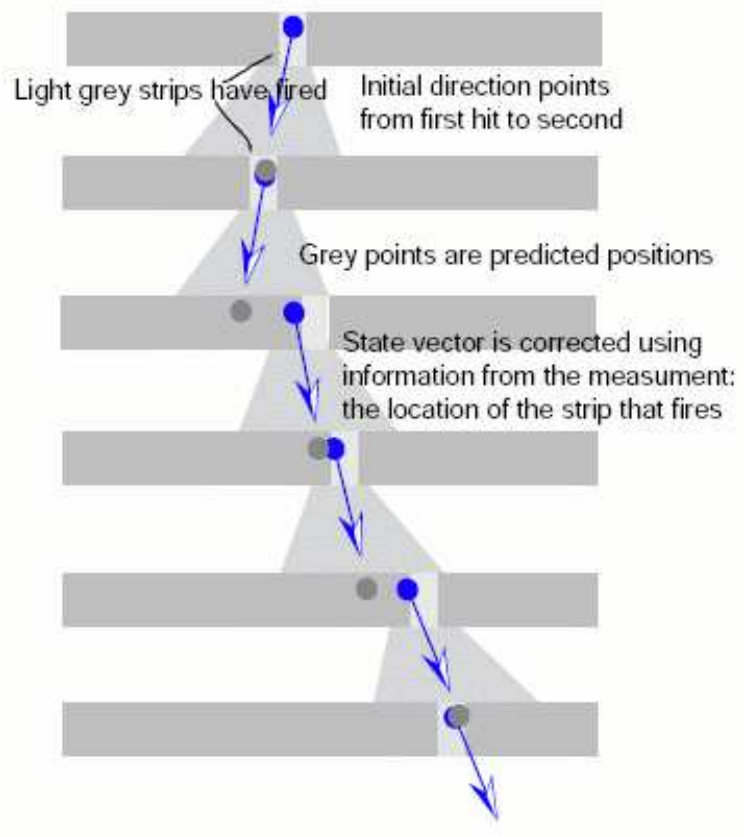

Figure 3.21: The Kalman filtering process. From [127].

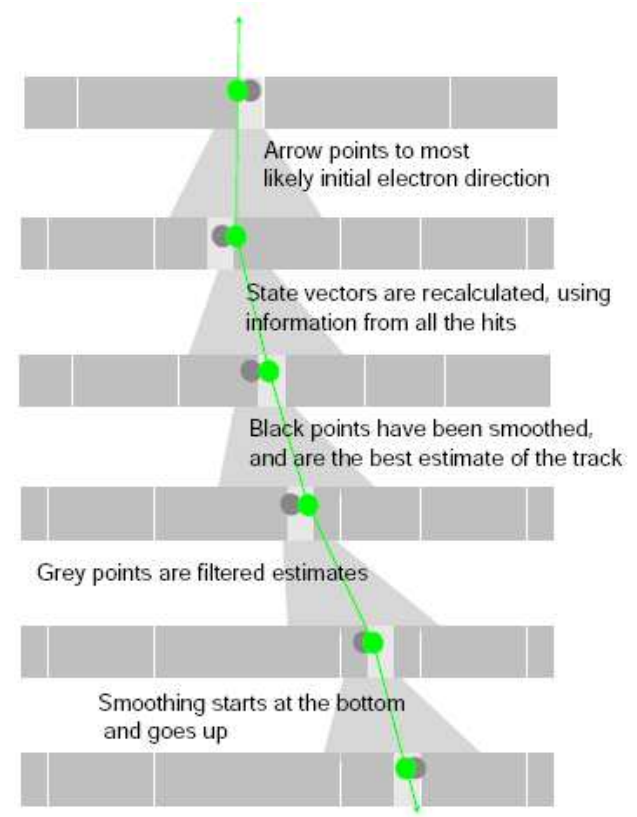

Figure 3.22: The Kalman smoothing process. From [127] 
of the track parameters, namely direction and energy, is available. Since the Kalman filter is somewhat computer intensive, it is only applied to the best-quality tracks, as determined by the track finding algorithm.

\section{Refined Energy Determination}

The fitted tracks and raw energy in the CAL are now used to provide a good estimate of the incident particle energy. The algorithm propagates the tracks across the calorimeter, determines the number of traversed radiation lengths, and corrects for edges and leakage through the back of the CAL. The measured raw energy and energy centroid are combined with this analytical expectation to determine the CAL incident energy.

Independently, the energy deposited in the tracker is estimated from the number of TKR hits ${ }^{17}$. The energy lost in the TKR is then added to the energy estimate from the CAL to determine the total incident energy. In practice, the TKR energy only constitutes a significant fraction of the total energy for low energy events (e.g. at $100 \mathrm{MeV}$ the tracker energy is $\sim 50 \%$ of the total, while at $1 \mathrm{GeV}$ it is $\sim<5 \%$ ).

It should be noted that the energy value reported at this stage of the event reconstruction algorithm is not final. The final energy analysis of LAT events will be described in the next section.

\section{Candidate Gamma-ray Event Reconstruction}

In this step, the current estimate of the incident energy is used to weight the hits in the track fitting routines and the tracks are thus recalculated.

The event reconstruction software uses the information from the tracks to define $\gamma$-ray candidates. For low energy events $(E \lesssim 1 \mathrm{GeV})$, determining the direction and

\footnotetext{
${ }^{17} \mathrm{~A}$ TKR hit represents an average energy loss of $\sim 0.6 \mathrm{MeV}$ if the hit happens in the thin section of the TKR, $\sim 1.97 \mathrm{MeV}$ on the thick section, and $\sim 0.35 \mathrm{MeV}$ on the empty trays
} 
energy of the $\gamma$-ray candidate involves reconstructing the vertex of the $\mathrm{e}^{-}-\mathrm{e}^{+}$tracks (even if the initial opening angle is small, multiple scattering will cause the separation to increase). For high energy events, the $\mathrm{e}^{-}-\mathrm{e}^{+}$pair will appear to make a single track that points back along the direction of the $\gamma$-ray.

Another important piece of information is obtained by projecting the tracks back to the ACD tiles and calculating the point of impact. If the track points clearly to an ACD tile (or ribbon) and that tile is hit, the event is very likely a background event. This is the same principle used by the hardware trigger veto and the on-board filter, however, the knowledge of the track direction at this stage is much more precise, and thus, this type of background rejection is a lot more powerful.

Apart from obvious quantities like event energy and direction, the event reconstruction software calculates hundreds of variables that completely describe the event as seen by the different sub-systems and the whole instrument. These quantities, known as ntuple variables, will be used during event selection and background rejection analyses (described below) to define a subset of "good" events. These are events that are classified as $\gamma$-rays (as opposed to background), and whose reconstructed energy and direction (and respective uncertainties) are expected to be well determined.

\subsection{Event Selection and Background Rejection}

\subsubsection{Energy Analysis}

Because of the vast phase space of the LAT, no single method is expected to provide the best energy reconstruction over all possible event interactions (particle angle, energy, etc.). Instead, the LAT collaboration has developed three independent approaches for energy reconstruction, as described below: 
- The parametric correction is the method explained in the last section to calculate the CAL energy during event reconstruction. This method can be applied to any event, and thus, provides a floor from which to improve.

- The likelihood method calculates the event energy using the observed correlation between energy deposition in the last calorimeter layer and the leaked energy. This method can only be used on those events with energy leakage through the back (bottom) of the calorimeter, and thus, its applicability is limited.

- The profile fitting method calculates the energy by fitting layer by layer the shower profile to an analytical description of the shower development in the calorimeter. This method works best when the shower peak is contained within the calorimeter.

The problem of choosing the best method for each event has been solved with the use of a data-mining technique known as classification trees [34]. In the context of LAT analysis, classification trees are used to predict the class to which an event belongs based on its ntuple variables. By training on Monte Carlo simulations, classification trees discover the event properties that make an event member of a given class, and the subtle correlations between those properties (event variables). In the case at hand, classification trees consider event properties (ntuple variables) such as raw energy and incoming angle to predict which energy method is best for each particular event.

The classification tree algorithm also calculates for each event the probability that the energy was well-reconstructed. Classification trees accomplish this by learning what circumstances make an event rather hard to reconstruct (e.g. the calorimeter misses a significant fraction of the particle energy because of the angle of incidence). For events with those characteristics the classification tree will yield a low probability for successful reconstruction. 
Classification trees offer a powerful tool to reject events whose energy is poorly measured, and thus, can be used to improve the LAT energy resolution at the expense of photon detection efficiency. The compromise between energy resolution and $\gamma$-ray acceptance is set by choice and depends on the special needs of the science topic under consideration. The baseline (default) energy resolution of the LAT instrument will be introduced in section 3.6.1.3.

\subsubsection{PSF Analysis}

The single-photon angular resolution is given by the angular distance between true and measured incident directions, and thus, it is used to characterize the angular resolution of the instrument (PSF). The measured direction of each event is given by the best track found during event reconstruction. However, many low energy events $(E<1 \mathrm{GeV})$ have a good vertex solution (combination of two tracks) in addition to the best track.

The LAT PSF is improved by using classification trees in a very similar way to the energy analysis described above. This time classification trees are used to: i) decide on whether or not to use the vertex solution, and ii) yield the probability that the track was well reconstructed.

The classification tree analysis takes into consideration the distinction between thin and thick events ${ }^{18}$, event topology (best track vs. vertex) and the PSF dependence on event energy (from multiple scattering). Independent classification trees are required for each one of the possible categories (e.g. thick-vertex-LowEnergy) to ensure proper classification.

Once again, the compromise between good PSF and $\gamma$-ray acceptance is set by

\footnotetext{
${ }^{18} \mathrm{As}$ discussed in section 3.2.2.2, the LAT TKR is divided into thin and thick sections. Depending on the energy, tracks from $\gamma$-ray events that convert in the thin section experience less multiple scattering at the beginning of the track than those that convert in the thick section. Since the initial hits are critical for determination of the track direction, thin events have a better PSF.
} 
choice and depends on the special needs of the science topic under consideration. The baseline (default) PSF of the LAT instrument will be introduced in section 3.6.1.2.

\subsubsection{Background Rejection}

Before proceeding with the discussion of background rejection in the ground, it should be noted that background rejection actually takes place in three different stages:

1. Hardware trigger veto

2. On-board filter

3. Event selections on the ground

As discussed in the previous section, the hardware trigger and the on-board filter make use of rather simple event quantities and their purpose is mostly operational (reduction of deadtime and compliance with the downlink bandwith). Background rejection on the ground is a lot more powerful since it can be done with sophisticated analysis tools and computational resources that are not available on orbit.

A detailed description of the LAT background rejection analysis is beyond the scope of this Chapter. Nevertheless, a brief description of the involved steps is given here for completeness:

1. Events are divided into categories determined by their topology (vertex or best track), energy, and location (thin or thick)

2. A specific set of selection cuts is applied to each category, followed by a classification tree that yields the probability that the event is background

3. Surviving events are now analyzed globally:

(a) with selection cuts, and 
(b) with a final classification tree

Not all background events are eliminated with the current background rejection analysis (which is under constant improvement). The rate and composition of the residual background obtained with a simulation that included over 5 billion background events is illustrated in fig. 3.23. Although there is still room for improvement, a special class of residual events are known to be a source of irreducible background (i.e. they cannot be eliminated by event selection). Typical irreducible background events include decay of pions produced in the blanket by cosmic ray protons, positron annihilation in the blanket, and $\mathrm{e}^{-}-\mathrm{e}^{+}$bremsstrahlung, also in the blanket. Since these reactions: i) occur outside the ACD, and ii) produce $\gamma$-rays within the LAT field-of-view, there is no way to distinguish these background-originated photons from astrophysical $\gamma$-rays. The rate of residual background events is very small for many GLAST observations. Nevertheless, the LAT collaboration has devised strategies to systematically subtract the irreducible residual contamination from the measured diffuse spectrum, namely, determination of the incoming particle fluxes $\left(\mathrm{e}^{-}, \mathrm{e}^{+}\right.$, protons) with LAT data and other satellite experiments (like PAMELA [219]), followed by MC simulations to infer the level of contamination.

The background rejection analysis provides the flexibility to fine tune the final selection according to the specific science analysis priorities. Nevertheless, a baseline implementation of background rejection has been defined for the LAT, which is adequate for most scientific analysis (including the one regarding this dissertation). The next section describes the instrument performance obtained with this implementation. 


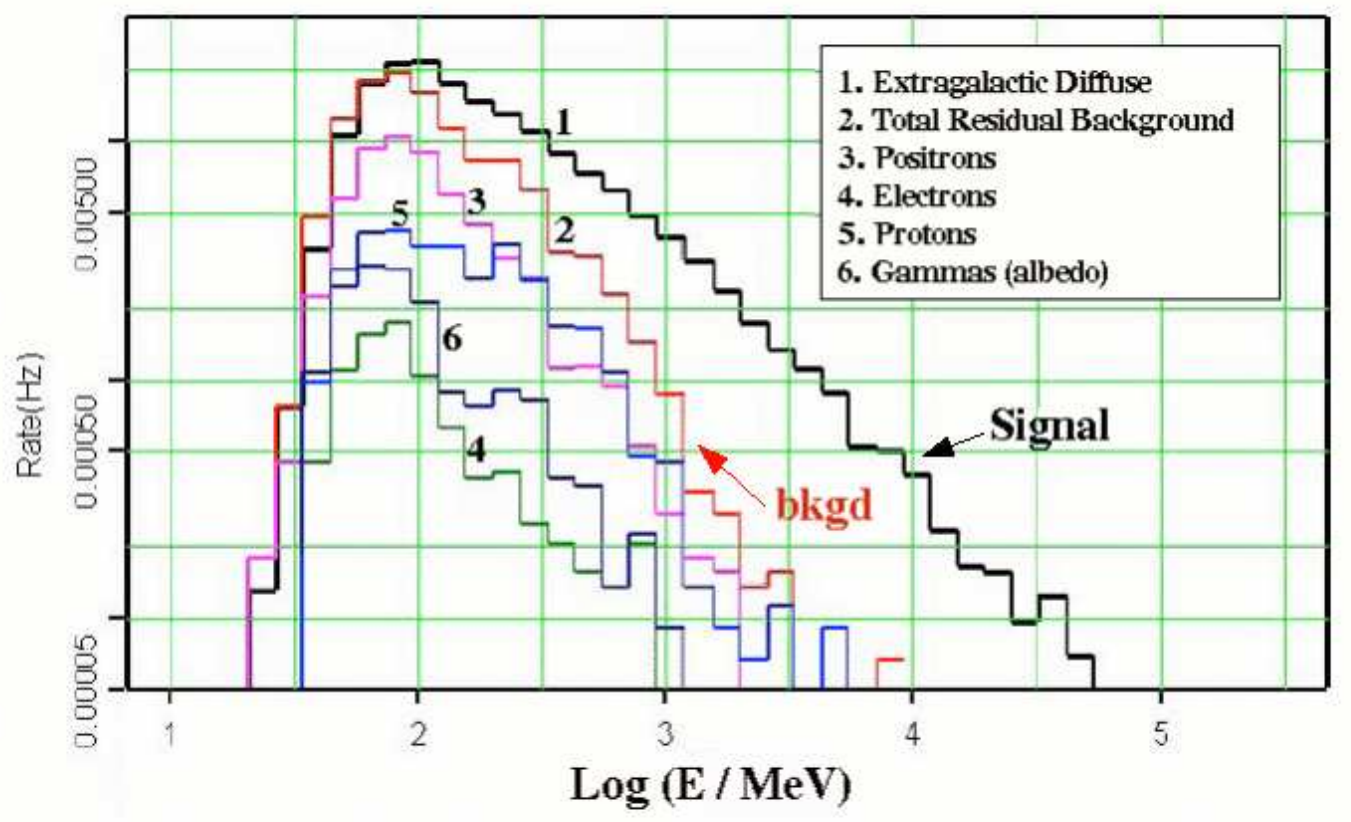

Figure 3.23: Residual background and extragalactic $\gamma$-ray diffuse rate as a function of energy. Adapted from [11] (for a background probability rejection cut of $P_{\text {background }}>$ $0.5)$. The extragalactic diffuse flux $\left(1.5 \times 10^{-5}\right.$ photons $\left.\mathrm{cm}^{-2} \mathrm{~s}^{-1} \mathrm{sr}^{-1}\right)$ is used as a benchmark for background rejection. Residual background (curve 2) consists mostly of positrons (curve 3) and protons (curve 5). Electrons (curve 4) and albedo gammas (curve 6) also contribute. 


\subsection{Brief overview of Instrument Performance}

\subsubsection{Instrument Response Functions (IRFs)}

The LAT instrument performance ${ }^{19}$ is determined by the interplay of the three elements below, all of which have already been discussed in this Chapter.

- Hardware design

- Event reconstruction algorithms

- Background rejection and event quality selections

A set of choices regarding the trade-off between $\gamma$-ray acceptance, event quality and background contamination has been made to produce a particular instrument performance that is adequate for most science topics. This standard instrument performance is introduced here through instrument response functions (IRFs) that describe the response of the instrument as a function of photon energy, incidence angle, conversion point within the instrument, and other important parameters [96].

The angular resolution for photons that convert in the thin section of the LAT is intrinsically better than those that convert in the thick section because the electronpositron pair are detected by the silicon detectors before significant multiple-scattering -which scales with the material thickness- has taken place.

The standard instrument performance comes in three flavors:

- Class $\boldsymbol{A}$ is the result of an analysis aimed at the extragalactic diffuse gammaray flux measurement, which is the most challenging for background rejection. For that analysis, $\gamma$-ray acceptance is sacrificed to obtain the purest sample.

\footnotetext{
${ }^{19}$ The instrument performance is under continuous improvement. The results presented here are subject to change. See [96] for an up to date description of the instrument performance.
} 
- Class $\boldsymbol{A l l}$ is the appropriate instrument performance for science topics that do not require a high level of background rejection. The residual background fraction in this class is substantially higher.

- Class $\boldsymbol{A}$ thin is the most restrictive of the classes. It offers a very pure $\gamma$-ray sample with superb angular resolution. It is obtained by selecting only those Class $A$ events that converted in the thin section of the TKR.

\subsubsection{Effective Area}

The first instrument response function to be considered is effective area. The LAT effective area reflects the final $\gamma$-ray detection efficiency of the instrument, which results from the total geometric acceptance, the conversion probability, and the detector and reconstruction efficiencies. The actual signal observed from a source is thus given by the convolution (over phase space) of flux and effective area. The effective area can be parametrized in terms of the photon energy (see fig. 3.24) or the incident angle (fig. 3.25) among others.

\subsubsection{Point-Spread Function (PSF)}

The point-spread function (PSF) gives the angular resolution of the instrument (angular distance between true and measured incident directions). It is usually expressed in terms of the angle for $68 \%$ (or 95\%) containment versus true photon energy as shown in fig. 3.26. It can be seen in fig. 3.26 that, as mentioned before, the PSF depends strongly on the photon energy $(\sim 1 / E)$ due to the multiple-scattering, which is higher at low energies. 


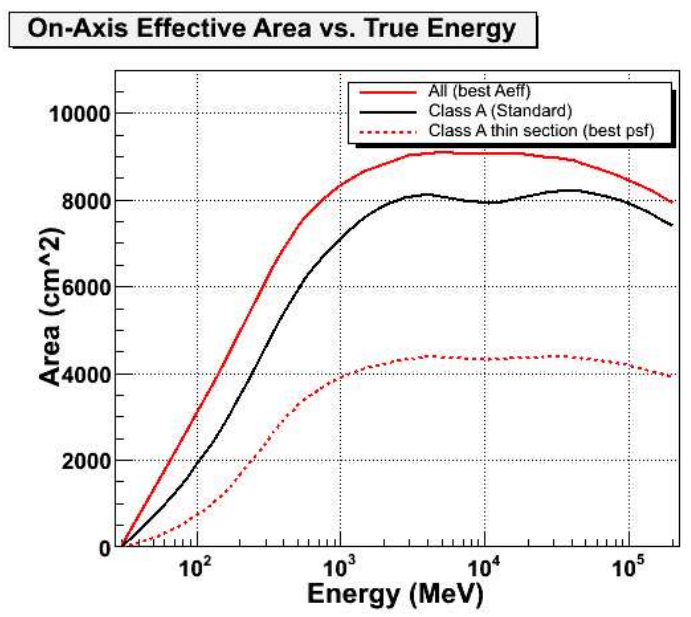

Figure 3.24: LAT on-axis effective area as a function of photon energy after selections for class all (solid red line, top curve), class $A$ (black line, middle curve) and class $A$ thin (red dashed line, bottom curve). Since the effective area at low energy is strongly influenced by background rejection selections, the LAT collaboration is studying a significantly looser set of selection cuts to increase the effective area below $1 \mathrm{GeV}$ for science topics such as gamma-ray bursts observation which require far less background rejection level.

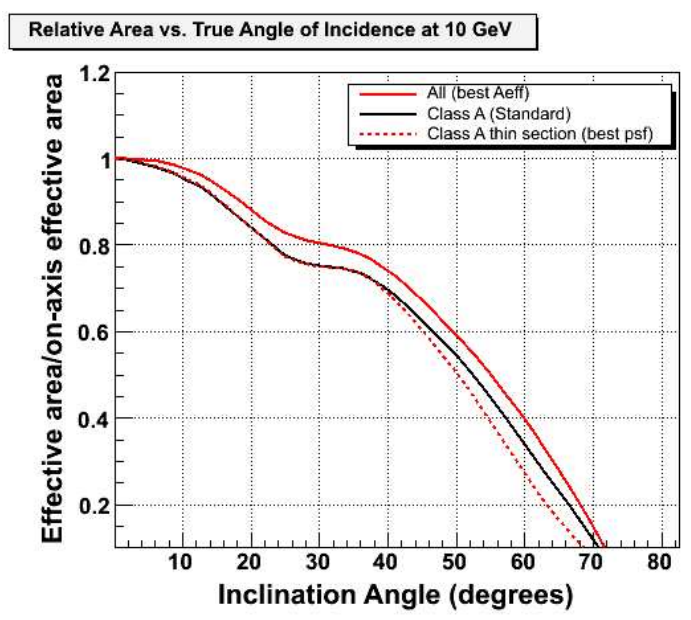

Figure 3.25: Relative effective area vs photon true angle of incidence for $10 \mathrm{GeV}$ photons. Effective area for class all (solid red line, top curve), class A (solid black line, middle curve) and class $A$ thin (dashed red line, bottom curve) are shown. The inflection at 25 degrees is an artifact of the parametrization. 


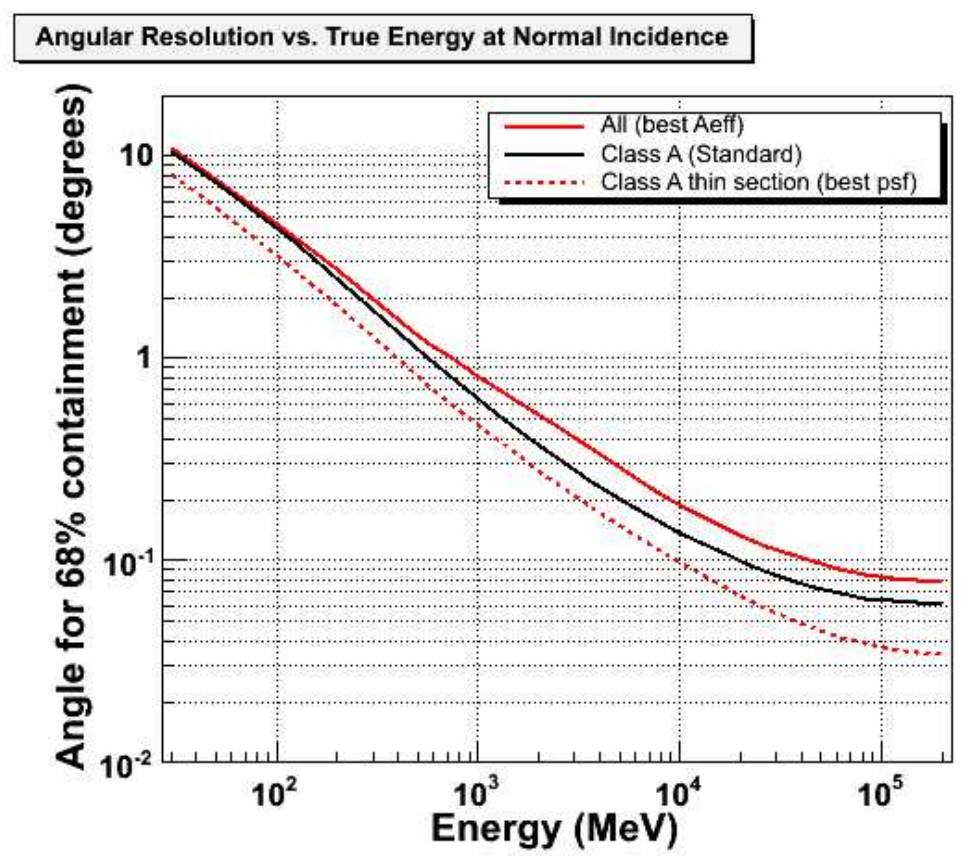

Figure 3.26: 68\% containment PSF versus true photon energy for class $A$ (black, middle curve), class all (red solid, top curve), and class A thin (red dashed, bottom curve).

\subsubsection{Energy Resolution}

The energy resolution $\Delta E / E=\left(E_{\text {Recon }}-E_{M C}\right) / E_{M C}$ (see fig. 3.27) measures the capability of the instrument to reconstruct correctly the energy of the incident $\gamma$ ray energy (which can be seen in fig. 3.28). Independently of the method used to reconstruct the event energy, the energy resolution is limited by the random nature of energy deposition of the shower, instrumental effects that are rather independent of the energy deposition (noise, pedestal dependence on temperature, etc.), and the systematics of energy correction (leakage, dead material, gaps) and shower modeling.

\subsubsection{Point-Source Sensitivity}

The LAT performance can now be used to calculate the sensitivity of the instrument to a point-like $\gamma$-ray source as a function of the source flux and observation time. This point-source sensitivity can then be used to predict for given conditions if a source 


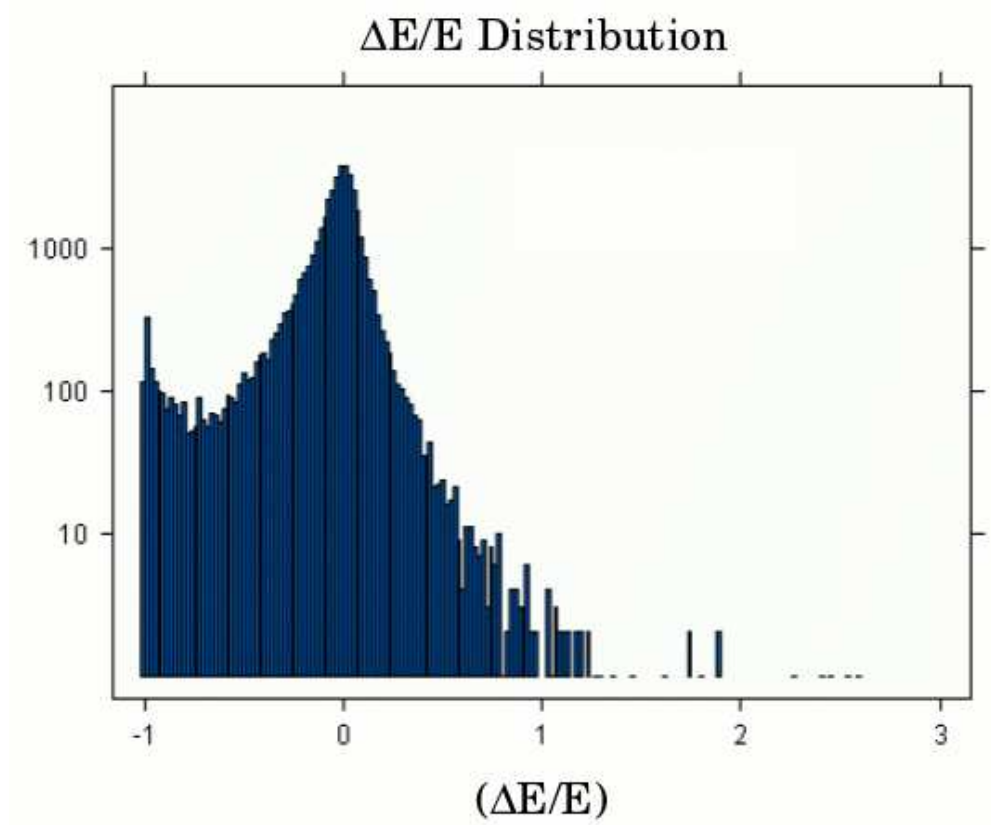

Figure 3.27: Energy resolution $\Delta E / E=$ abs $\left(E_{\text {Recon }}-E_{M C}\right) / E_{M C}$ over the LAT energy range after background rejection and energy resolution cuts. From [13].

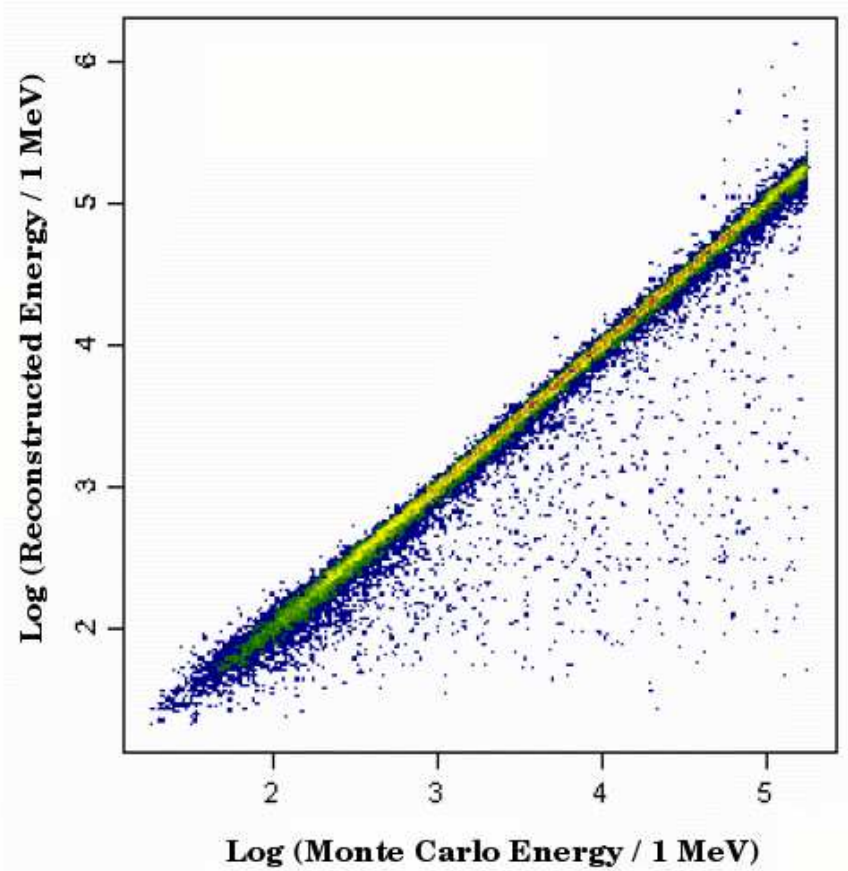

Figure 3.28: Reconstructed vs Monte Carlo Energies after background rejection and energy resolution cuts. From [13]. 


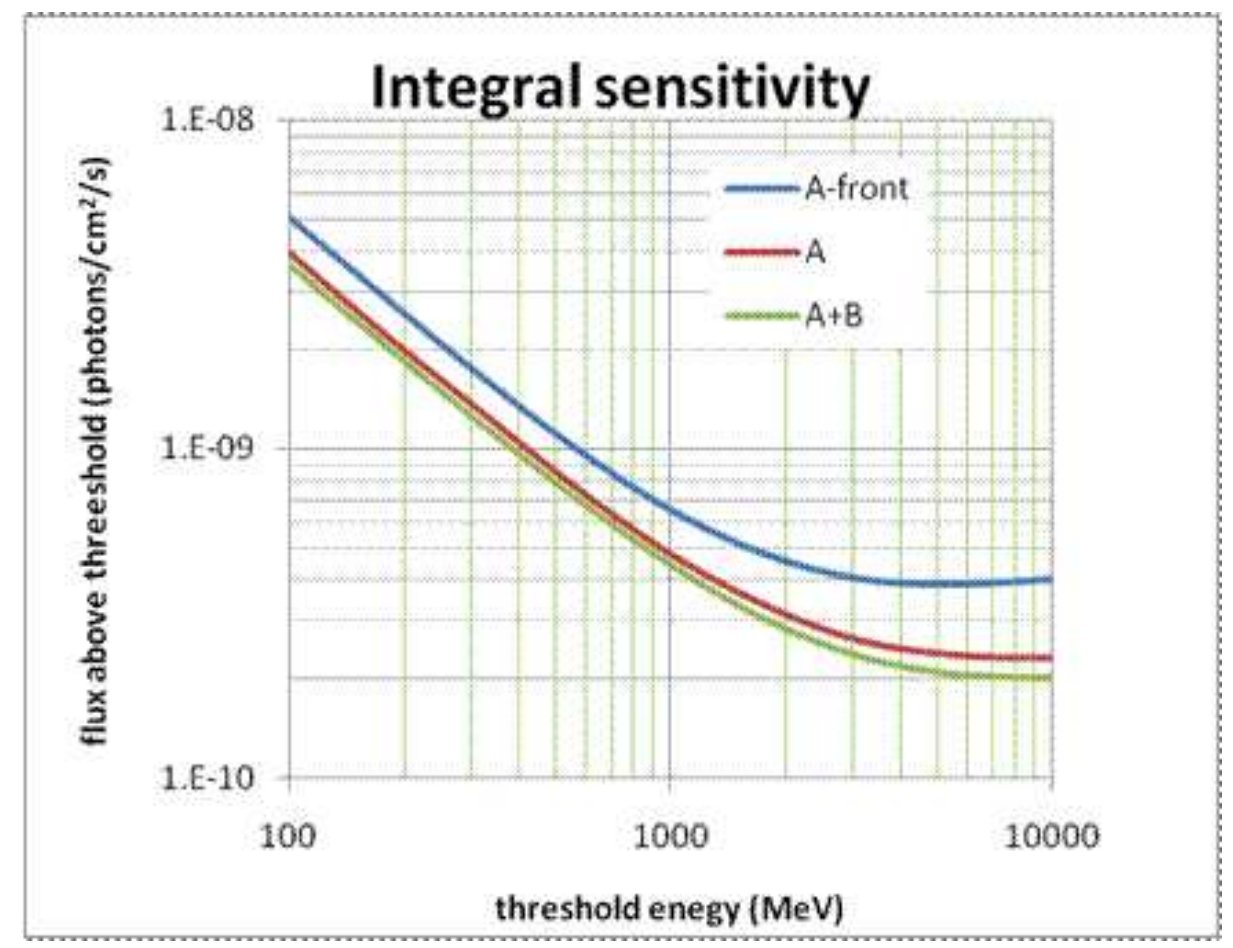

Figure 3.29: GLAST integral point-source sensitivity from [96] for a point source with $E^{-2}$ spectrum and diffuse background flux of $1.5 \times 10^{-5} \mathrm{~cm}^{-2} \mathrm{~s}^{-1} \mathrm{sr}^{-1}$. The red solid line represents the 1-year, $5 \sigma$ integral sensitivity of GLAST as a function of integral flux (y-axis) and threshold energy (x-axis). The 5-year curve is also shown in blue.

will be above detection threshold $(5 \sigma)$. The instrument point source sensitivity will be used in Chapter 5 to produce a reasonable prediction of the number of blazars that GLAST will detect according to given models of intrinsic blazar luminosity function, which is currently unknown, but will be measured by GLAST. Figure 3.29 shows the GLAST integral point-source sensitivity for a point source with $E^{-2}$ spectrum and diffuse background flux of $1.5 \times 10^{-5} \mathrm{~cm}^{-2} \mathrm{~s}^{-1} \mathrm{sr}^{-1}(E>100 \mathrm{MeV})$ [96].

The description of the LAT instrument and its performance is now concluded. Chapter 4 presents an analysis of the ACD backsplash effect as observed during the beam test at CERN of the LAT calibration unit in 2006. If left unmitigated, backsplash self-veto has the potential to greatly reduce the effective area of the instrument at high energies $(E>10 \mathrm{GeV})$, which is essential for EBL studies (Chapter 5). 


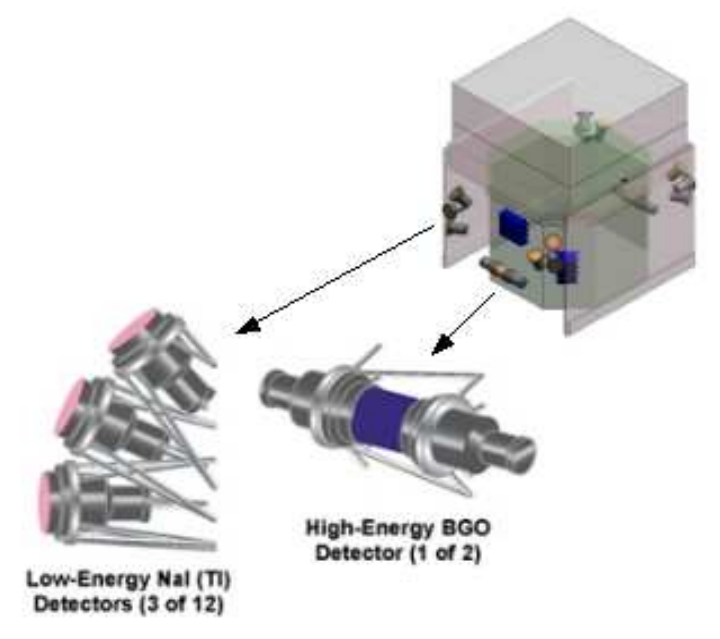

Figure 3.30: GLAST Burst Monitor. The $12 \mathrm{NaI}$ and 2 BGO scintillator detectors are located around the spacecraft pointing at different directions in order to maximize the spatial coverage.

\subsection{GLAST Burst Monitor (GBM)}

The second instrument on board GLAST is the GLAST Burst Monitor (GBM) [273, 185], whose primary purpose is to detect gamma-ray bursts (GRBs) over a very large field of view $(8-10 \mathrm{sr})$ in the energy range between $10 \mathrm{keV}$ and $\sim 25 \mathrm{MeV}$. Since the GBM is not designed to study AGN (and their attenuation by the EBL), only a brief description is given here for completeness.

The GBM consists of $12 \mathrm{NaI}$ and 2 BGO scintillation detectors (described in sec. 1.1.2.1) located around the spacecraft pointing at different directions in order to maximize the spatial coverage (fig. 3.30). The NaI detectors are the workhorse of the GBM, they cover the lower part of the energy range (10 keV $-1 \mathrm{MeV})$ and are responsible for the burst triggers and source localizations. The BGO detectors cover the energy band $0.15 \mathrm{MeV}-\sim 25 \mathrm{MeV}$ and therefore provide good energy overlap between the NaI detectors and the LAT.

The GBM will detect and localize gamma-ray bursts (GRB) within its large field of view and then will provide prompt alerts to the LAT and to ground-based instruments. 
The spacecraft can slew towards the location of the GRB and measure its flux at higher energies. It should be noted that the LAT will also detect GRBs on its own, with onboard software that analyzes real-time data in search for transients. 


\section{Chapter 4}

\section{Beam Test of the LAT Calibration Unit: ACD Backsplash Studies}

\subsection{Beam Test Rationale}

Monte Carlo simulations are used to study the LAT instrument response and to develop and optimize reconstruction and background rejection algorithms that will be used during science operations. It follows then, that a thorough validation of the Monte Carlo tools is essential to the scientific success of the LAT.

Beam tests are used to verify and tune the Monte Carlo simulations. A beam test at SLAC (Stanford Linear Accelerator Center) in 1997 [15] used simple versions of the three subsystems (ACD, CAL, TKR) and its main goal was to verify the technology and design choices of the instrument. A second test in 1999/2000, also at SLAC [61], was performed with an engineering model very similar in design to one of the tower modules of the full instrument. It focused on system integration and validation of Monte Carlo simulations for on-axis and off-axis incident beams (positrons and tagged photons) of different energies up to $\sim 20 \mathrm{GeV}$.

The latest beam test was performed in 2006. The primary goal of this very comprehensive beam test was to examine the LAT performance with as-built flight hardware. 
Since the energy range and field-of-view of the LAT are vast, it is neither practical nor necessary to verify by direct comparison the full range of LAT performance with the beam test. Instead, the beam test is used to sample the performance space, so that more extensive and detailed analyses with simulated data can be used to characterize accurately the full instrument performance [14].

There are two particular areas where verification by this beam test are particularly important:

- Implementation in the simulation of the underlying physics of electromagnetic and hadronic interactions (as described by GEANT4 [91], a widely used suite of simulation routines)

- Instrument model (geometry and detectors response)

The analysis presented below of beam test data provides a Monte Carlo verification and characterization of one aspect of the instrument: the ACD backsplash effect. As explained in Chapter 3, backsplash from an incident high-energy photon $(\gtrsim 10 \mathrm{GeV})$ can produce ACD veto signals (self-veto effect), and thus reduce the acceptance of the instrument to high-energy events, which are essential to many scientific analyses, including EBL studies.

\subsubsection{ACD Backsplash}

The LAT has been designed to detect $\gamma$-rays with energies up to $>300 \mathrm{GeV}$, where any space-based detector of plausible size (effective area $\sim 1 \mathrm{~m}^{2}$ ) will begin to run out of photons due to the typical falling spectra of astrophysical $\gamma$-ray sources. Detection of photons at such energies with good energy resolution also requires a calorimeter that is deep enough to absorb much of the electromagnetic shower produced by the $\gamma$-ray after undergoing pair-conversion. For a given incident $\gamma$-ray, the profile and intensity of the shower depend on the amount of material in the calorimeter (measured in 


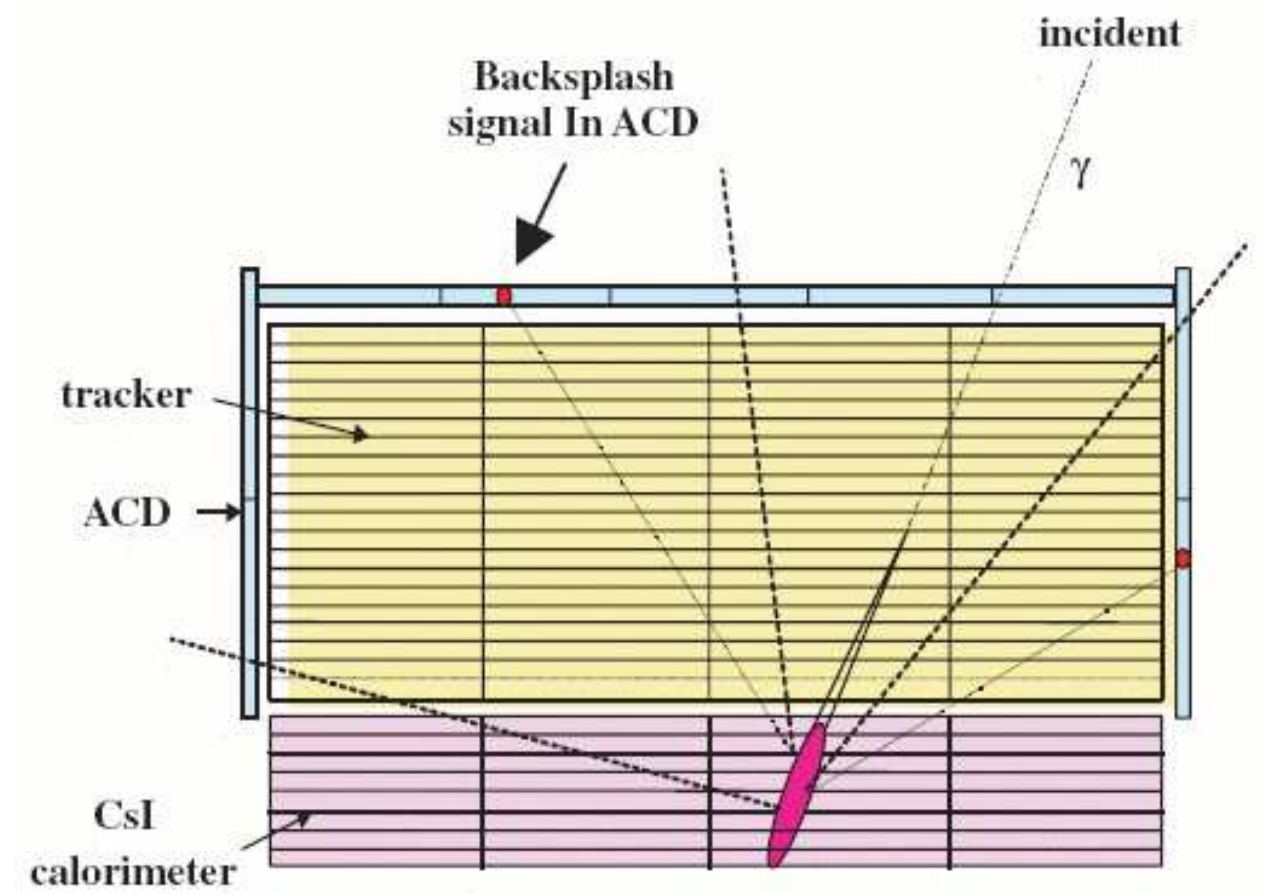

Figure 4.1: Schematic representation of ACD backsplash, from [191]. Incident $\gamma$-ray and backsplash particles are indicated. Signals in the ACD are shown by red dots.

radiation lengths) and the type of material itself (via atomic-number dependence of the electromagnetic processes).

Most of the charged particles and photons in the electromagnetic shower (sec. 1.1.1.2) travel along the direction of the incident photon, but a small fraction of them are emitted isotropically (mostly $100-1000 \mathrm{keV}$ photons). This radiation is known as backsplash. Backsplash radiation can create additional signals in the detectors above the calorimeter, namely, the TKR and the ACD (see fig. 4.1). The number and distribution of backsplash hits in the TKR have no net effect on the track fitting and reconstruction algorithm when added to those produced by the incident high energy $\gamma$-ray. On the other hand, the effect of backsplash in the ACD could be very significant. When backsplash photons cross the ACD tiles, they can interact with the material via Compton scattering and thereby produce scintillation light that is digitized and discriminated by the ACD electronics as described in Chapter 3 . If the 
signal produced by backsplash is over veto threshold, the gamma-ray event could be misidentified and rejected as background. EGRET with its monolithic ACD dome, was greatly affected by this effect. Its effective area for $10 \mathrm{GeV}$ photons was a factor of two lower than at $1 \mathrm{GeV}$ due to backsplash self-veto [201].

The LAT was designed to avoid this problem by using a segmented ACD. When a particle is incident on the instrument, only the ACD segment intersected by the backwards projected path of the particle is used to veto the event. Thus, the fraction of events that are self-vetoed is determined by the hit probability integrated over the relatively small area $\left(\sim 1000 \mathrm{~cm}^{2}\right)$ covered by an individual ACD tile (as opposed to EGRET, whose backsplash was determined by the hit probability integrated over the whole ACD dome).

The ACD hit probability per unit area as a function of energy and distance backwards from the shower has already been studied with simulations and beam tests for different calorimeter materials [191]. These studies were used to set the level of segmentation in the ACD design and to validate the design choices. The purpose of the ACD backsplash analysis presented in this Chapter is two-fold:

1. To study the backsplash probability with as-built flight detectors and readout electronics

2. To verify the capabilities of the Monte Carlo simulations to reproduce backsplash effect

\subsection{Beam Test Description}

\subsubsection{LAT Calibration Unit Description}

For this beam test the LAT collaboration built a calibration unit that consists of two complete flight spare towers (TKR $+\mathrm{CAL})$, an additional CAL module, five ACD 


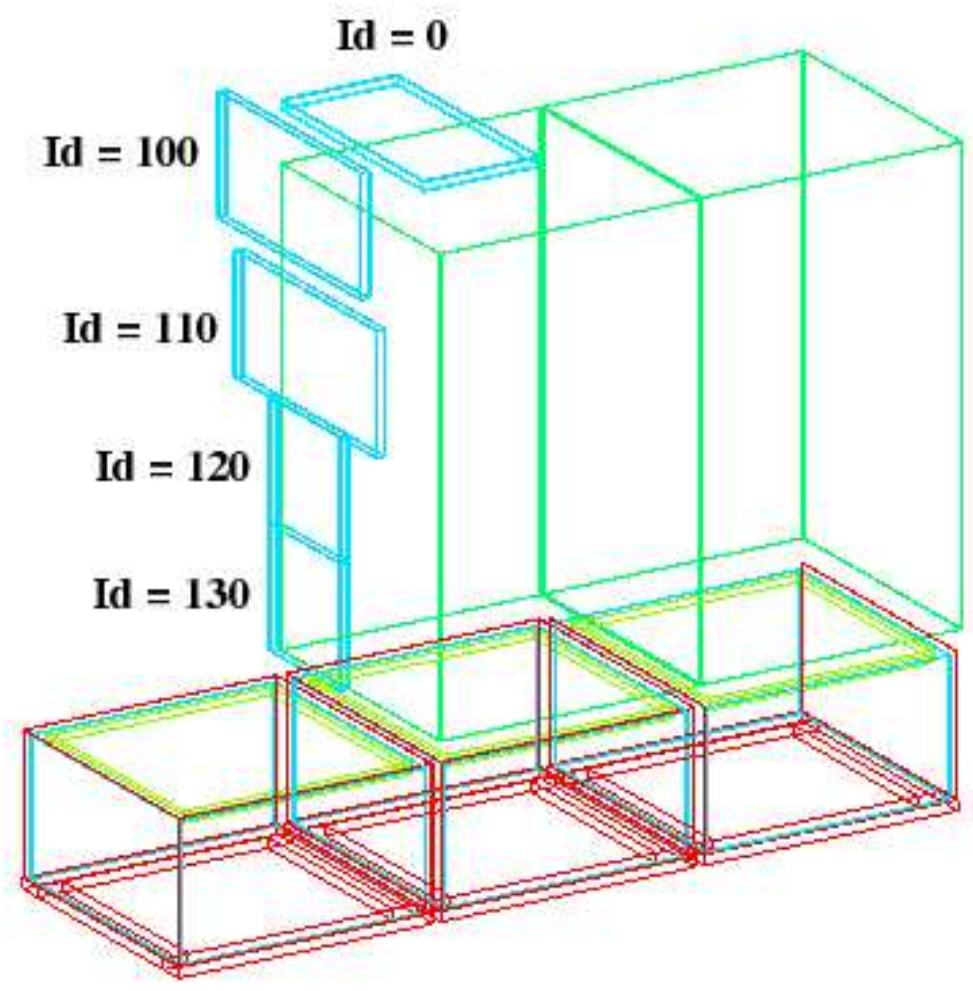

Figure 4.2: ACD tiles location with respect to the TKR (green) and CAL (red) modules. The ACD tiles shadows the adjacent tower in the same way that the ACD subsystem shadows the 16 towers of the LAT. 
tiles and flight-like readout electronics (calibration unit is shown schematically in fig. 4.2). This simplified version of the LAT allows for a comprehensive test of the event reconstruction since most of the LAT events will be contained within 2 towers.

The ACD tiles of the calibration unit are, for practical purposes, identical to the tiles in the LAT. They are made of the same material and have the same thickness, with the only difference being their size (see fig. 4.3 for dimensions). Large and small tiles were placed on the top and side of one of the towers to reproduce the shadowing effect that is characteristic of the LAT ACD as illustrated in fig. 4.2. The wavelength shifting fibers ( 1 m long), PMTs and Front-End Readout Electronics (see Chapter 3) are all identical to those used in the LAT.

As can also be seen in figs. 4.2-4.3, each tile is assigned a number id that follows a LAT geometry naming convention. The tile ids are kept here for convenience and they are from top to bottom: 0, 100, 110, 120, and 130. Tile 100 of the calibration unit has poor light collection uniformity due to known problems with its embedded fibers, and thus, will not be considered for this analysis.

\subsubsection{Beam Description}

The CERN (European Center for Nuclear Research) accelerator complex was chosen for this beam test because it can provide electron, photon, pion and proton beams with energies high enough to fully probe the LAT energy range (up to $\sim 280 \mathrm{GeV}$ ). The GLAST beam test at CERN was organized in two different runs, one at the PS (Proton Synchrotron) accelerator and a second one at SPS (Super Proton Synchrotron). The experimental lines at PS offer $e^{-}, p^{+}, \pi$ and tagged photon beams with energies up to a few $\mathrm{GeV}$. The H4 line at SPS, where the second part of the experiment took place, is regarded as the best experimental line at CERN because of its well-defined, clean and low energy-dispersion particle beams. Electron, proton and pion beams were obtained at this facility with energies ranging from $10 \mathrm{GeV}$ to $280 \mathrm{GeV}$. Because backsplash is 

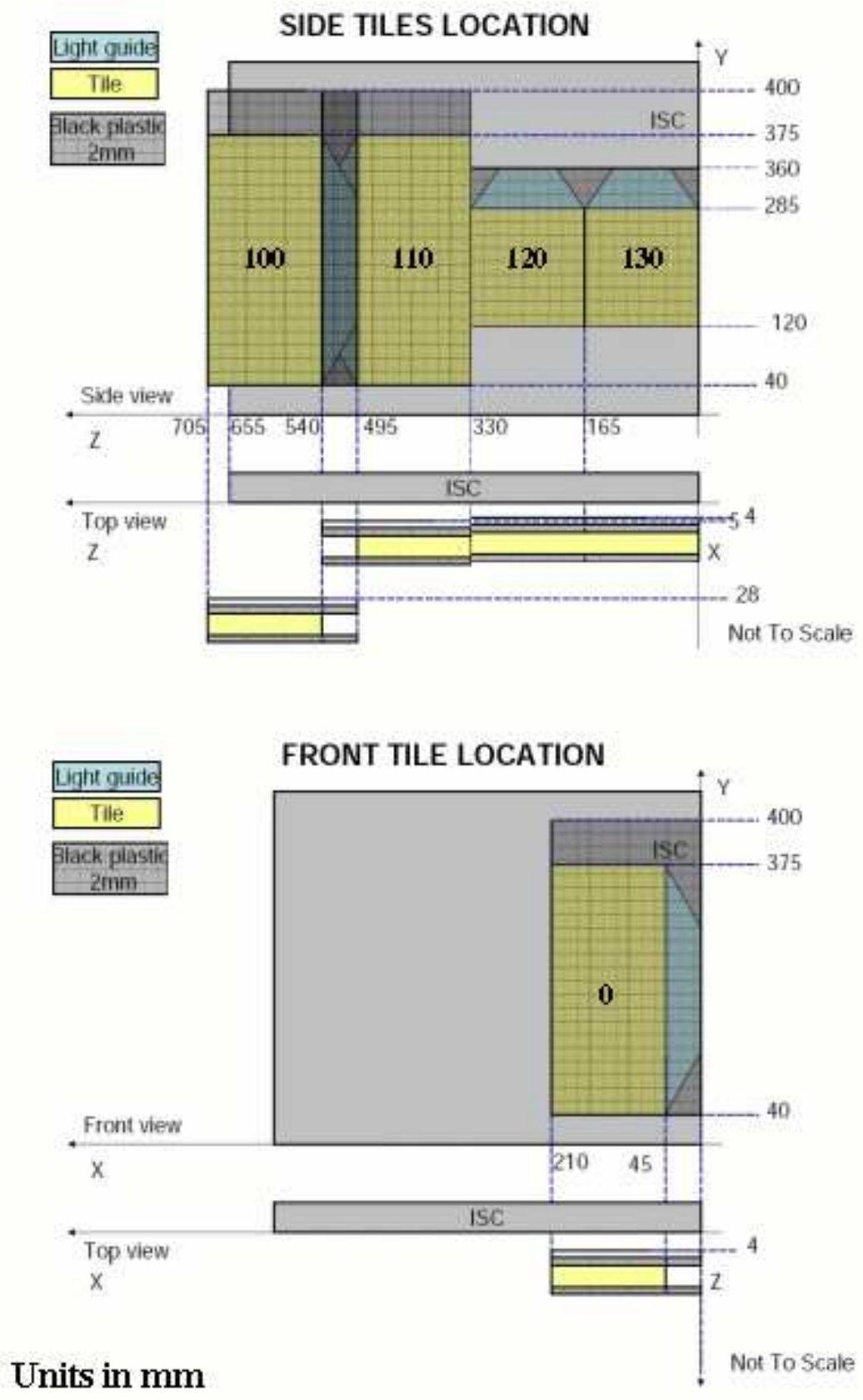

Figure 4.3: Dimensions of the ACD tiles in the LAT calibration unit. Courtesy of the beam test group. 


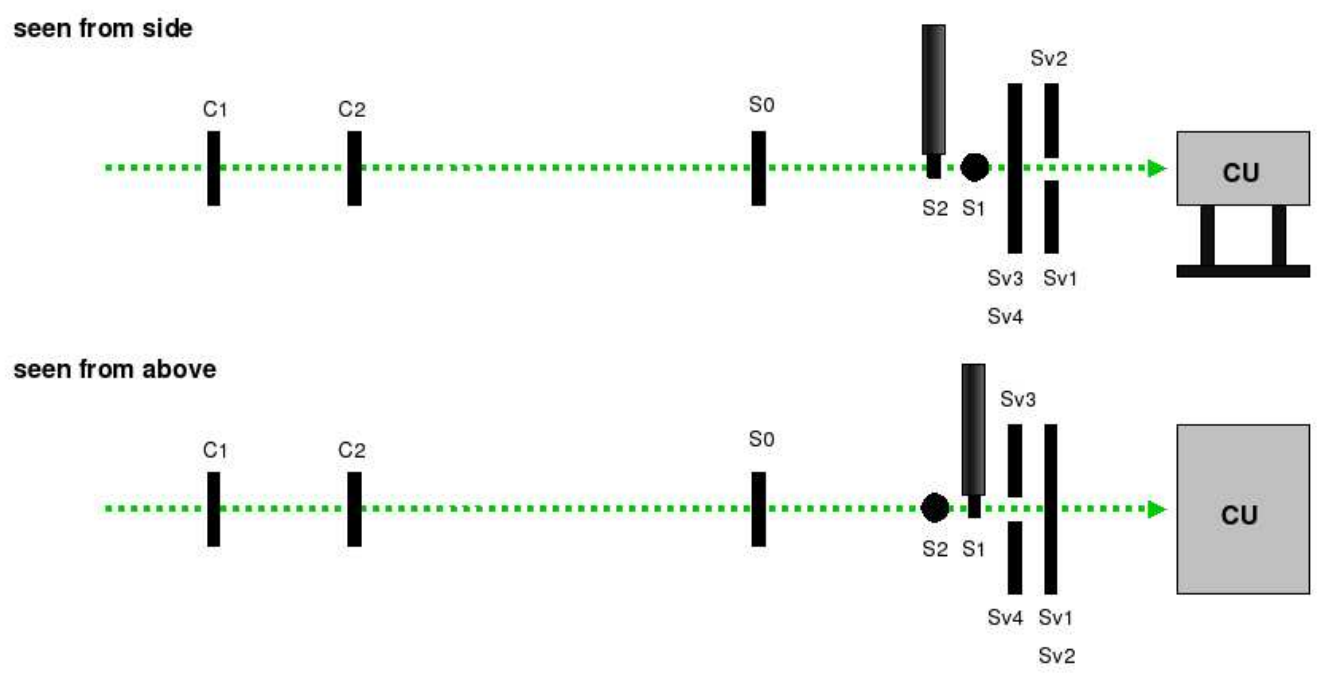

Figure 4.4: Side and front view of the experimental setup at SPS. Courtesy of the beam test group.

significant at high energy depositions, only data taken at SPS is considered for this analysis.

\subsubsection{Experimental Setup}

The experimental setup at SPS is shown schematically in fig. 4.4. In addition to the beam line and the calibration unit, a system of scintillators provided an external signal that was used (when appropriate) to trigger the instrument. The optional use of Cherenkov detectors along the beam line provided particle discrimination for proton and pion beams. Beam events were selected by the coincidence of two of the detectors (S1, S2) and the anti-coincidence of the remaining four (SV1, SV2, SV3, SV4). The detectors location and trigger logic ensured a narrow beam profile $(\sim 2 \mathrm{cmx} 2 \mathrm{~cm})$ with small angular dispersion. 


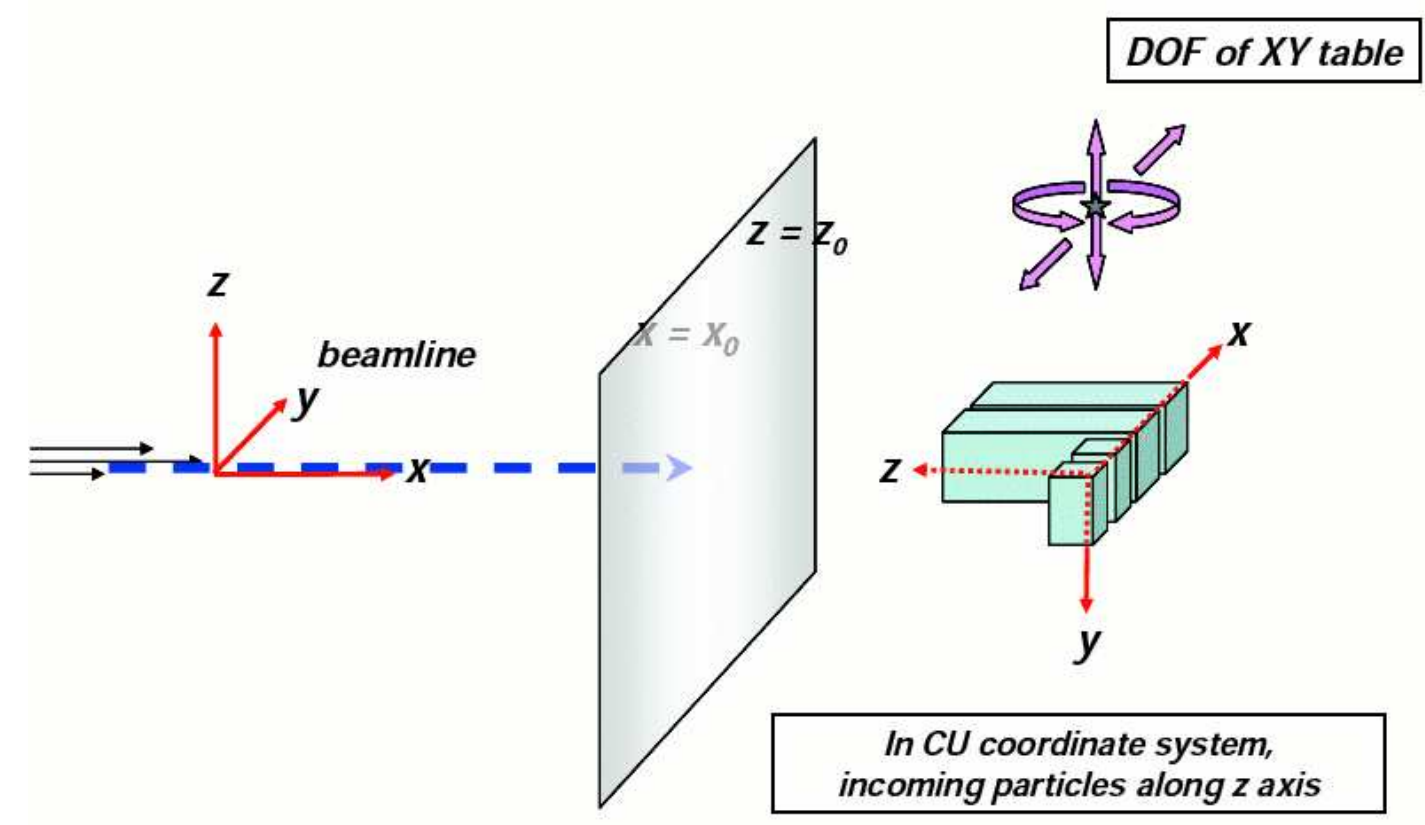

Figure 4.5: Orientation of the calibration unit with respect to the beam direction. The calibration unit is placed in a moving table which was controlled remotely. The table had 3 spatial degrees of freedom $(\mathrm{x}, \mathrm{y}, \mathrm{z})$ and one rotational degree of freedom in the XZ plane $(\theta)$. Courtesy of the beam test group. 

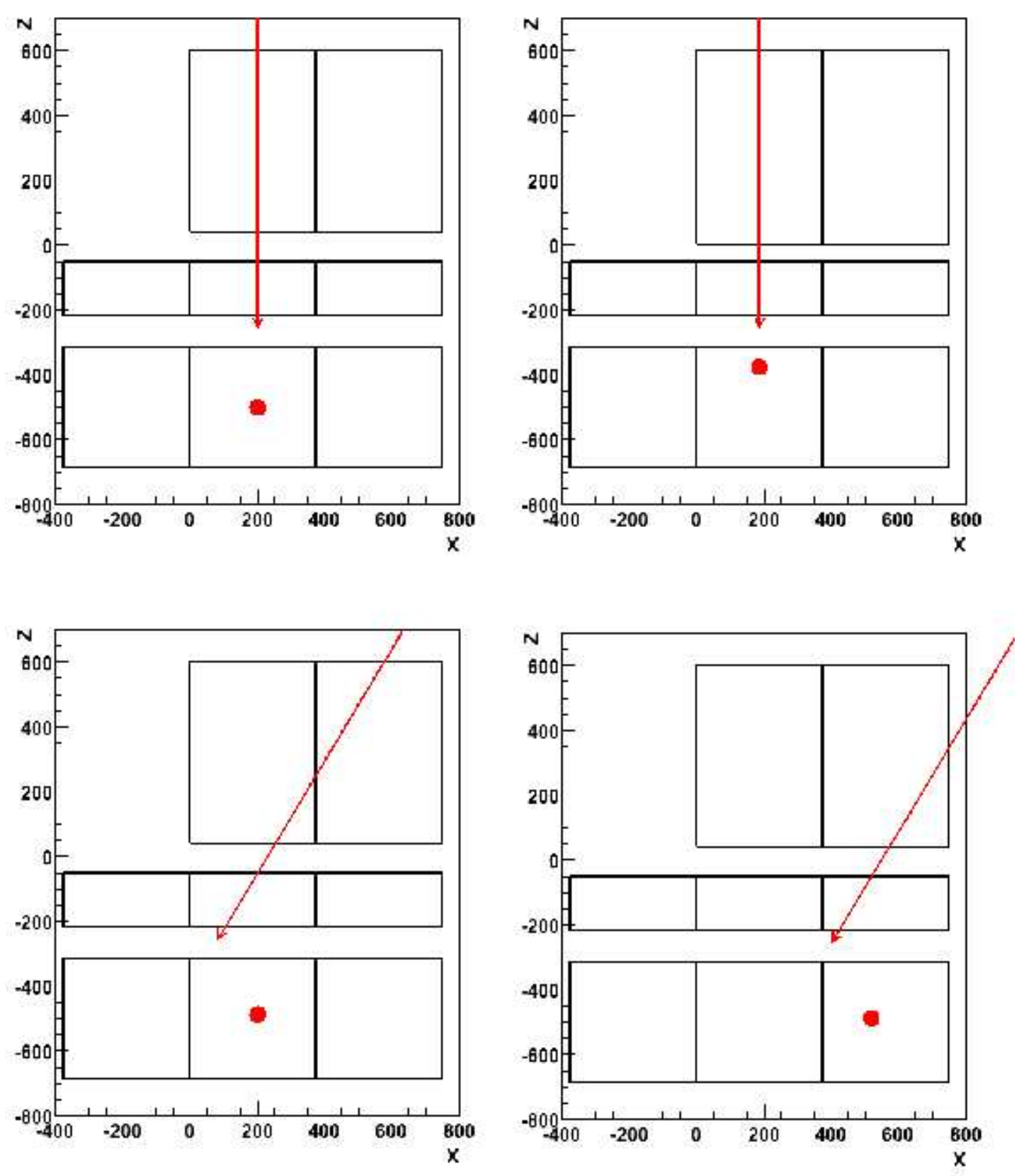

Figure 4.6: Calibration unit during different data taking configurations. Courtesy of the beam test group. Each panel contains a schematic of the calibration unit as seen from the top and from the side. The schematic at the top of each panel indicates the beam trajectory (red line), while the figure at the bottom indicates the impact point of the beam with respect to the three calorimeter modules. From the panels it can be seen that the impact point and angle of incidence can be changed at will. Over 300 different configurations were used during the beam test. 


\subsubsection{Data Taking Configurations}

The calibration unit was placed on a moving table with the TKR towers in a horizontal position and thus parallel to the beam. The table had two spatial degrees of freedom and one rotational degree of freedom $\theta$, as shown in fig. 4.5. This allowed the beam to hit the calibration unit at different impact points and with different angles of incidence, as illustrated in fig. 4.6.

The freedom to choose different particle beams (electrons, protons, pions), with different energies $(10-280 \mathrm{GeV})$, incident with different angles $\left(-90^{\circ}-90^{\circ}\right)$ and impact points, permitted us to sample the LAT phase space with over $\sim 300$ data taking configurations, which are summarized in table 4.1 .

Although the ultimate goal of this analysis is to understand the effects of backsplash for high energy photon events, electrons are used instead since photon beams are not available at the relevant energies at CERN or any similar facilities. This does not diminish the validity of the results since the differences between showers initiated by electrons and photons are well understood (as discussed in Chapter 1) and considered in the simulations [74]. The different data configurations used during the beam test with high energy electrons are summarized in table 4.2.

\subsection{Analysis}

\subsubsection{Considerations}

The signal output from an ACD tile is determined by the following processes (in sequential order):

- The amount of light produced in the scintillator tile by the incident particle

- Light collection by the wavelength shifting fibers (WLS; described in Chapter 3) 
- Light attenuation in the fibers

- Poisson fluctuations in the number of photoelectrons produced in the PMT

- Noise fluctuations in the pulse digitization

The amount of light produced in the tile is proportional to the particle energy loss by ionization, and thus is described by the Bethe-Block formalism [19]. It is well known that the energy fluctuations in such processes are characterized by a Landau distribution [74]. In the LAT simulation this is calculated with GEANT4.

The light collection efficiency of the ACD tiles has been studied during the design, building, and testing phases of the instrument [190]. During the design phase, the use of WLS fibers was found to provide the best light collection efficiency, and the space between fibers was optimized to achieve good uniformity ${ }^{1}$ over the tile area. This uniformity has been measured for the LAT ACD tiles, and it was found that the light collection is uniform in the central area of the tile (fluctuations of the order of $\sim 10 \%$ or less) and that it decreases towards the tile edge. The reduction factor by which the light collection decreases near the edge is different tile to tile, but in general, it was found that the light collection could be as bad as $\sim 70 \%$ at the tile edge, and recovers back to $100 \%$ when measured $\sim 3 \mathrm{~cm}$ away from the edge [190].

The light collected in the tile is delivered to the PMTs through fibers, which in the case of the calibration unit, are located $\sim 0.4$ to $1 \mathrm{~m}$ away, depending on the tile. Although a significant fraction of the light is lost during transport ( $\sim 20 \%$ for $1 \mathrm{~m}$ long fiber), light attenuation is not expected to induce significant fluctuations in the signal and thus its effect is absorbed into the channel calibration.

When the collected light is delivered to the PMT, the light hits the PMT cathode and liberates electrons via the photoelectric effect. These photoelectrons are acceler-

\footnotetext{
${ }^{1}$ Good uniformity is defined as the absence of large fluctuations in the light collection over the tile area. The design goal for the LAT ACD was to have a light collection efficiency in any central region of the tile that is within $10 \%$ of the tile average.
} 
ated and guided by electric fields to impinge on a secondary metallic plate, or dynode, which multiplies the number of electrons by an average factor (or gain), that is equal to 5 in the case of the LAT PMTs. This multiplication process is repeated for a total of 10 times to generate a sufficient number of electrons for voltage measurement in the external circuit. The number of photoelectrons produced at every stage is governed by Poisson statistics, and when considering all the stages involved in the ACD output, Poisson fluctuations in the number of photoelectrons have the largest statistical impact on the observed ACD signal distribution, especially when the number of photoelectrons is low $\left(N_{p e}<5\right)$.

Finally, the ACD electronics contribute to the width of the distribution with a low level of noise, which was estimated during calibration.

\subsubsection{ACD Calibration}

As the first step of the analysis, the response of every ACD tile was calibrated with data obtained during special runs. In the first run, the pedestal of every ACD channel was observed by triggering the instrument with zero-suppression off. The observed pedestal distribution in ADC counts for tile 0 is shown in fig. 4.7. As can be seen from the plot, the pedestal distribution is well fitted by a gaussian function. The same procedure was performed for all the tiles and the resulting mean values and Gaussian widths are summarized in table 4.3.

Afterwards, every tile was exposed to a $150 \mathrm{GeV}$ proton beam at normal incidence. The beam was aimed at the center of the tile where the light collection is uniform, and thus, no edge effects are expected. The pulse-height distribution obtained with the proton beam is shown in fig. 4.8 for tile 0 . The energy loss of protons passing through matter is well known from experiment and for the case at hand $(150 \mathrm{GeV}$ proton traversing a $1 \mathrm{~cm}$ thick plastic scintillator at normal incidence) follows a Landau distribution whose mean value is equal to $\sim 2.2 \mathrm{MeV}$ [74]. A minimum- 


\begin{tabular}{|c|c|c|c|}
\hline Particle type & Energy $(\mathrm{GeV})$ & Angle $\left(^{\circ}\right)$ & Events \\
\hline \hline electrons & $\begin{array}{c}10,20,50, \\
100,200,280\end{array}$ & $\begin{array}{c}0,10,20,30, \\
\pm 45, \pm 60, \pm 90\end{array}$ & $\sim 17.7 \mathrm{M}$ \\
\hline protons & $20,100,150$ & $\begin{array}{c}0,30,45,60,-51, \\
\pm 90\end{array}$ & $\sim 0.8 \mathrm{M}$ \\
\hline pions & 5,20 & $0,-90$ & $\sim 2.2 \mathrm{M}$ \\
\hline \hline & & Total: & $\sim 20.7 \mathrm{M}$ \\
\hline
\end{tabular}

Table 4.1: Data configurations during the beam test at CERN SPS in 2006.

\begin{tabular}{|c|c|c|}
\hline Energy $(\mathrm{GeV})$ & Angles $\left(^{\circ}\right)$ & Number of Events \\
\hline \hline 10 & $0,10,20,30,45,60$ & $\sim 1.2 \mathrm{M}$ \\
\hline 20 & $0,10,20,30,45,60$ & $\sim 2.7 \mathrm{M}$ \\
\hline 50 & $0,10,20,30,45,60$ & $\sim 2.5 \mathrm{M}$ \\
\hline 100 & $0,10,20,30,45,60$ & $\sim 3.5 \mathrm{M}$ \\
\hline 200 & $0,10,20, \pm 30, \pm 45, \pm 60$ & $\sim 3.8 \mathrm{M}$ \\
\hline 280 & $0,10,20,30,45,60, \pm 90$ & $\sim 4 \mathrm{M}$ \\
\hline \hline & Total: & $\sim 17.7 \mathrm{M}$ \\
\hline
\end{tabular}

Table 4.2: Electron runs at SPS.

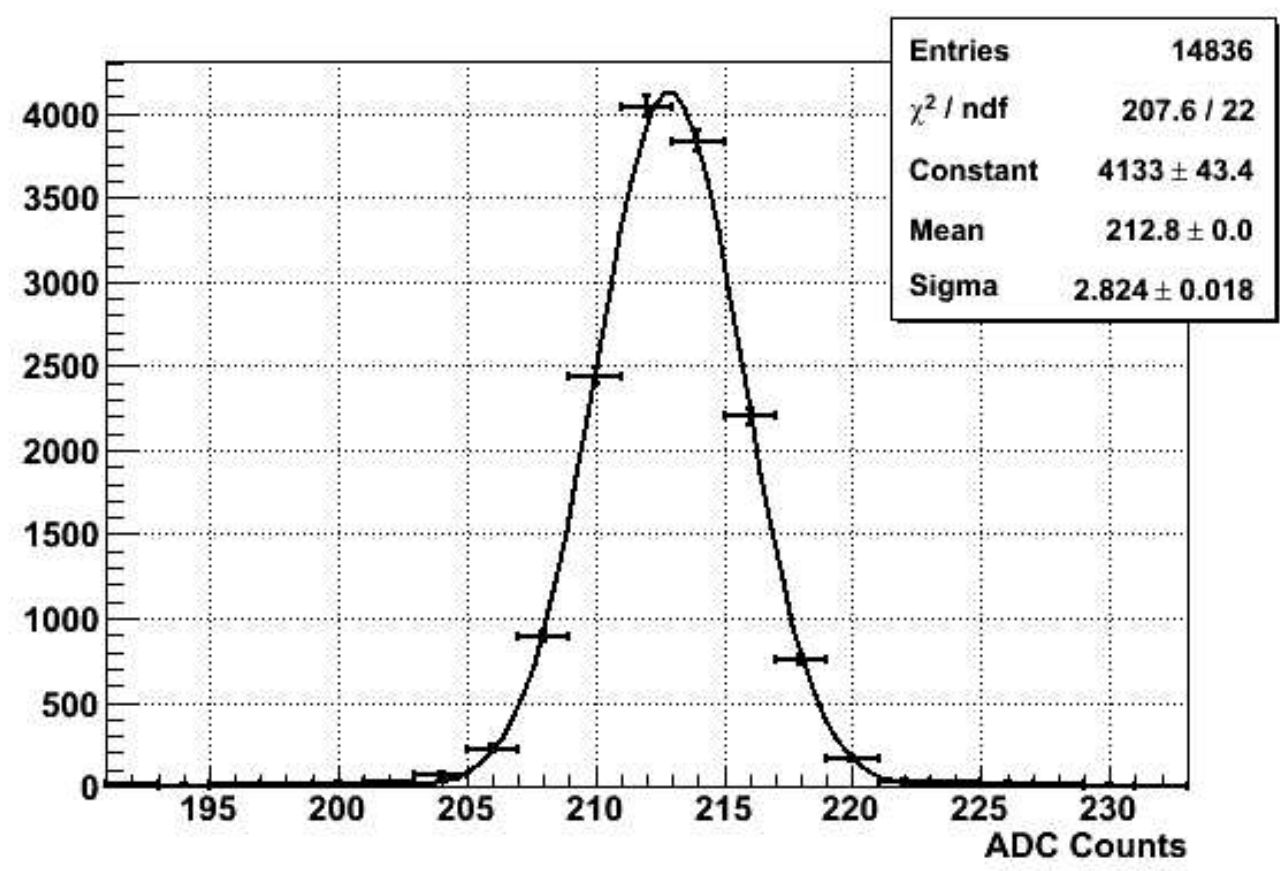

Figure 4.7: Pedestal for tile 0 in ADC counts. The pedestal distribution can be approximated by a Gaussian function with mean equal to 212.8 and sigma equal to $2.82 \pm 0.02 . \mathrm{Th}$ 


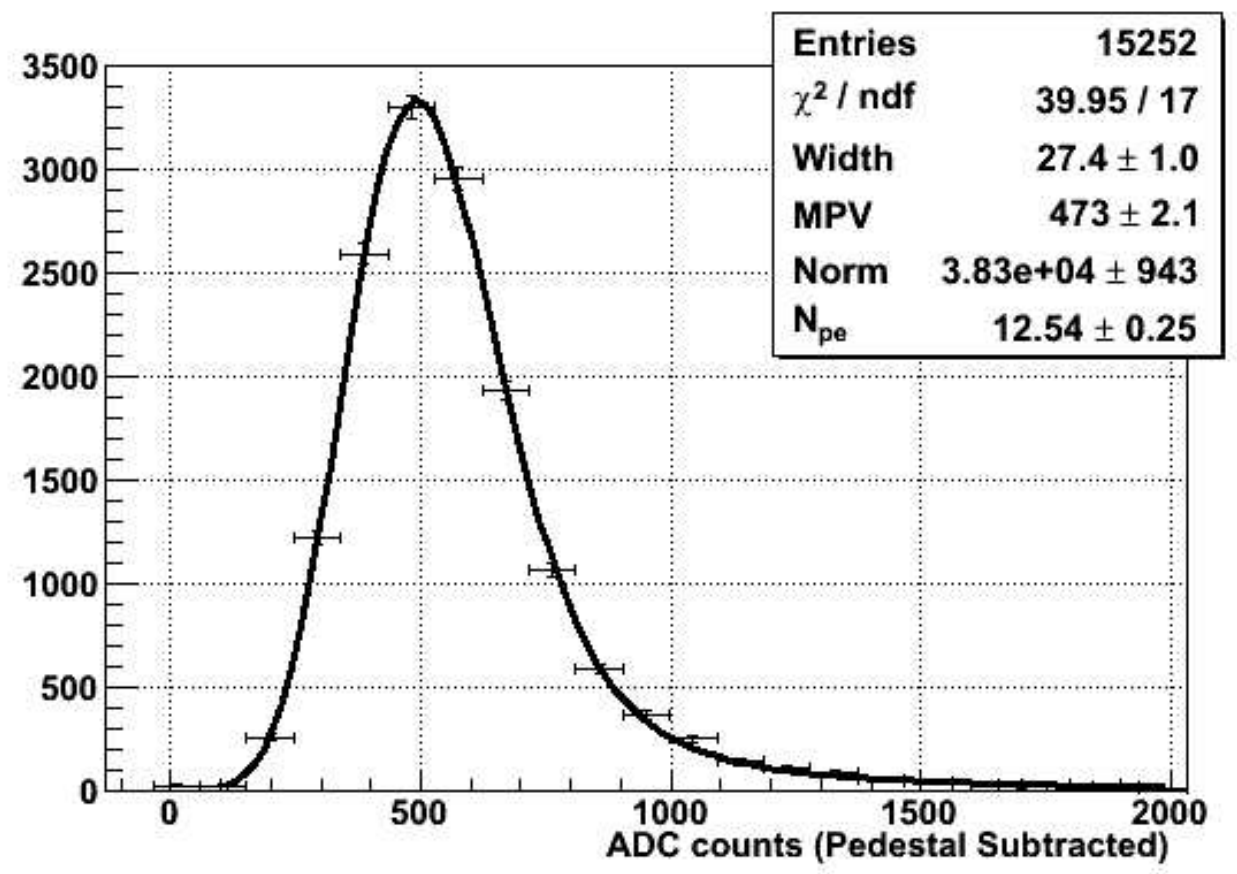

Figure 4.8: Pulse-height spectrum on ADC counts for tile 0 exposed to a $150 \mathrm{GeV}$ proton beam. The MIP peak position is determined by fitting the histogram to the convolution of Landau (energy fluctuations) and Poisson (number of photoelectrons) distributions.

ionizing particle (MIP) meanwhile, crossing the same tile at normal incidence would deposit $1.9 \mathrm{MeV}$. The latter value is a natural scale for the energy deposition in the detector and hereafter will be used as a measurement unit and referred to as 1 mip.

The gain on each ACD channel was determined by fitting the pulse-height histogram obtained with the proton beam to the convolution of Landau and Poisson distributions, as can be seen in fig. 4.8. The free parameters of the fit are:

- Width (or sigma) of the Landau distribution

- Most Probable Value (MPV) of the Landau Distribution

- Normalization Constant

- Mean number of photoelectrons $\left(N_{p e}\right)$ for an energy deposition of $2.2 \mathrm{MeV}$ (i.e. 1.2 mips) 
The peak position, the full width at half maximum (FWHM), the mip position in $\mathrm{ADC}$ units and the mean number of photoelectrons per mip were obtained from the fitted distribution. These values are summarized in table 4.4. The ratio of Landau width to FWHM ( $\sim 0.3$ on average) reveals that the relative magnitude of the Landau fluctuation is not dominant and that most of the signal width is due to Poisson fluctuations in the number of photoelectrons.

ACD calibration provides very important quantities for this analysis:

- The mip position and pedestal values of every channel will be used in the following sections to express the energy deposition in every tile in units of mips

- The mean number of photoelectrons per mip will be used to simulate the Poisson fluctuations experienced by the ACD signals

- The Gaussian width of each pedestal distribution will be used in the simulation to account for fluctuations due to electronics noise.

\subsubsection{Measured Backsplash Distribution}

The backsplash distribution measured for a $200 \mathrm{GeV}$ electron beam is considered in this section to illustrate the analysis procedure. For this particular run, the calibration unit was oriented at $0^{\circ}$ with respect to the beam as indicated in fig. 4.9 , with the impact point set to the center of the tower.

\subsubsection{Event Selection}

The event selection ensures that only high energy electrons coming from the beam are considered in the analysis. This is possible thanks to the external trigger provided by the ancillary system described in sec. 4.2 .3 and to the reconstruction and trigger information that is available for each event. 
The signals produced by the incident electron and its electromagnetic shower are digitized by the instrument and used to reconstruct the event in the same way that an event in the LAT instrument would have been reconstructed. This results in several low- to high-level variables that can be used to select a clean sample of events. In particular, the event selection requires that the particle track is consistent with the direction expected for the beam. The event selection cuts are summarized in table 4.5 .

The data acquisition system of the calibration unit gathers and reduces the trigger primitives from the different subsystems, making them available for analysis. Thus, beam events can be selected by requiring the coincidence (within $0.6 \mu \mathrm{s}$ ) of the external trigger (from the ancillary system) with the internal TKR 3-in-a-row trigger and $C A L$ low ${ }^{2}$ trigger of the calibration unit.

It should be noted that this event selection reduces the impact of background events only when the background particles can be detected with the instrument via the tracker or the calorimeter (cosmic muons that cross the instrument at the same time than the beam for example). The possibility remains however, that soft background $(E \lesssim 10 \mathrm{MeV})$ could be present in the experimental area, especially during beam operation. These types of particles would not be detected by the TKR or CAL but could mimic the effect of backsplash in the ACD tiles. This possibility will be considered in sec. 4.3.3.3.

\subsubsection{Results}

The ACD signal measured in tile 110 is shown in fig. 4.10. As expected, the backsplash spectrum falls quickly as a function of energy, and thus, it is very different from the one measured when a single charged particle crosses the detector (see fig. 4.8 for comparison). Ideally, backsplash self-veto could be reduced by setting a high veto

\footnotetext{
${ }^{2} 100 \mathrm{MeV}$ energy deposition in any of the CAL crystals (described in sec. 3.2.3.3)
} 
threshold. This however, is contrary to the high charged-particle detection efficiency that is necessary for background rejection at the trigger level (which requires a low veto threshold). Therefore, the veto threshold has been carefully tuned to optimize the instrument acceptance to high energy $\gamma$-rays (by reducing backsplash self-veto) while conserving the ability to reject background events (as described in sec. 3.5.3). The current nominal setting of ACD veto threshold is 0.45 mips as indicated in fig. 4.10 .

Hereafter, backsplash is calculated for every tile as the ratio of the number of events $n_{x}$ with energy deposition above a threshold $x$ to the total number of events $N$ :

$$
P_{b a c k s p l a s h}(x)=\frac{n_{x}}{N}
$$

If $P(x)$ is considered as a backsplash probability, then the probability of obtaining exactly $n_{x}$ backsplash hits in $N$ independent trials is given by the binomial distribution

$$
f\left(n_{x} ; N, P(x)\right)=\frac{N !}{n_{x} !\left(N-n_{x}\right) !} P(x)^{n_{x}}(1-P(x))^{N-n_{x}}
$$

with the variance of $n_{x}$ given by [74],

$$
\sigma_{n_{x}}^{2}=N P(x)(1-P(x))
$$

Thus, the statistical error of the measured $P(x)=n_{x} / N$ is given by,

$$
\sigma_{P(x)}=\sqrt{\frac{P(x) \times(1-P(x))}{N}}
$$

Figure 4.10 shows the obtained backsplash distribution $P(x)$ for tile 110 for the data run introduced above. Although the probability of having an energy deposition over 0.1 mips is high $(\sim 20 \%)$ for this tile, a veto threshold at 0.45 mips would result 


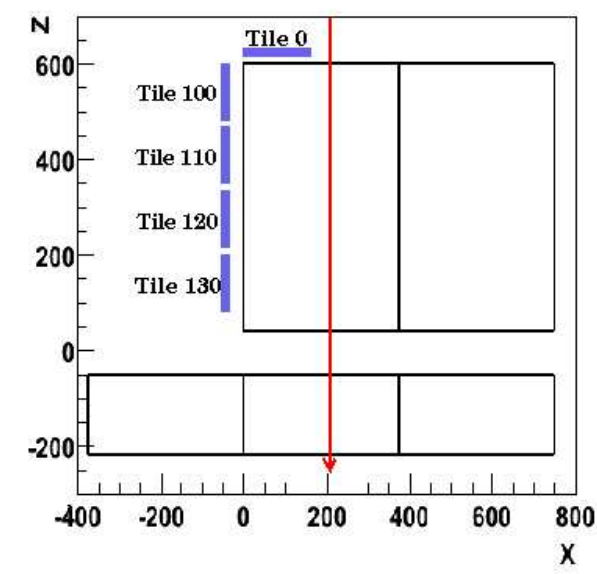

Figure 4.9: Calibration unit orientation with respect to the beam.

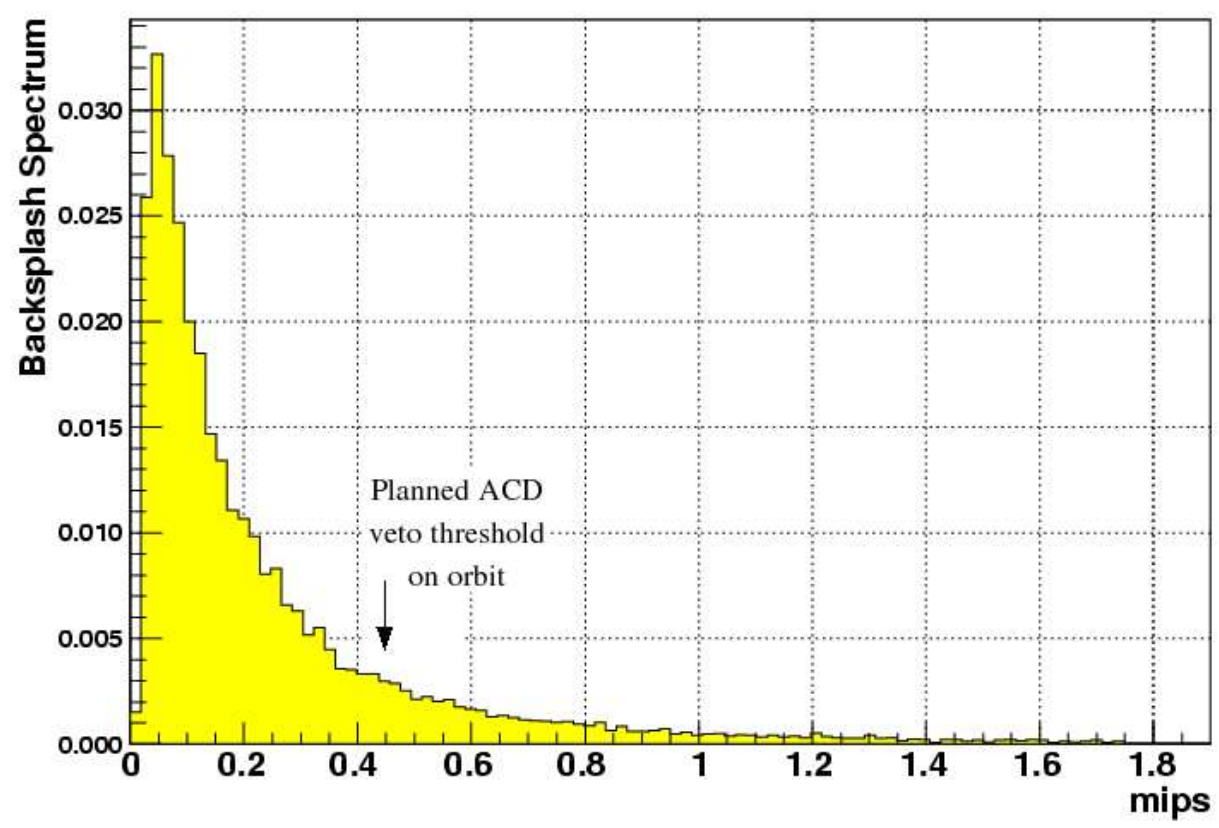

Figure 4.10: Measured ACD signal distribution in units of mips for tile 110, normalized to the total number of events in the sample $(\sim 75 \mathrm{k})$. These signals are originated by backsplash radiation from a $200 \mathrm{GeV}$ electron beam that is hitting the calorimeter as indicated in fig. 4.9 . 


\begin{tabular}{|c|c|c|}
\hline Tile & Pedestal Mean & Gaussian Width \\
\hline \hline 0 & 212.8 & $2.82 \pm 0.02$ \\
\hline 110 & 168.2 & $2.46 \pm 0.01$ \\
\hline 120 & 140.4 & $3.06 \pm 0.02$ \\
\hline 130 & 140.1 & $2.75 \pm 0.02$ \\
\hline
\end{tabular}

Table 4.3: Pedestal mean and width in ADC counts for every ACD tile.

\section{Tile 110}

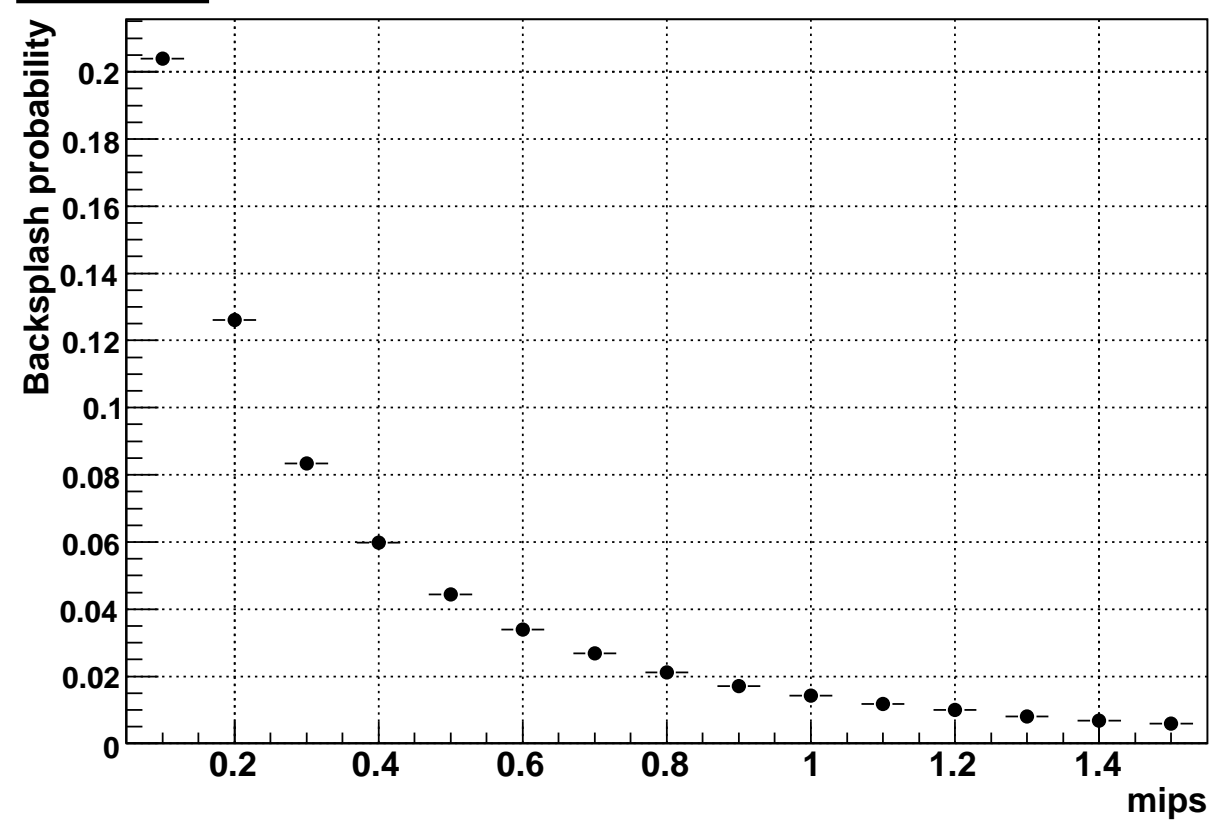

Figure 4.11: Measured backsplash probability for tile 110 for a $200 \mathrm{GeV}$ electron beam at normal incidence. Backsplash is calculated as a function of energy threshold with every data point being equal to the ratio of the number of events above the threshold to the total number of events. The statistical error bars $(1 \sigma)$ are small due to the large number of events $(\sim 75 \mathrm{~K})$. 


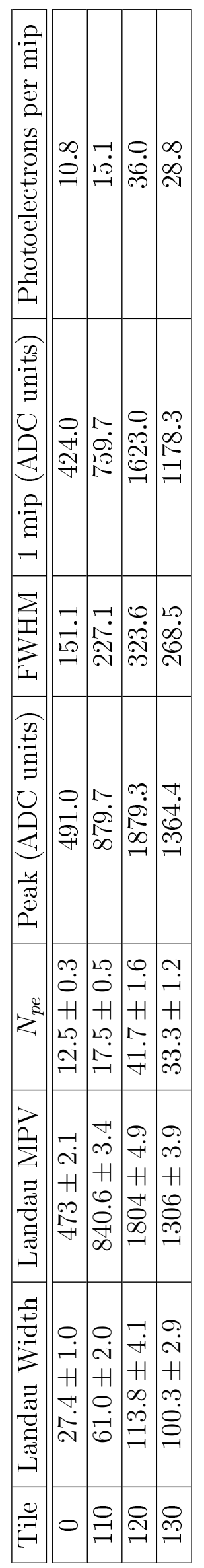

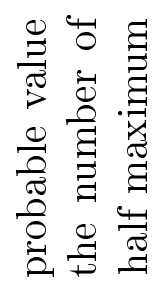

落.

丞芯

宅 异

ซี

击是

晃番

$\stackrel{\pi}{\ominus} . \Xi z^{\dot{\alpha}}$

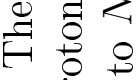

들

항

흥

용

氙苟

$\therefore$ a

웡 웅

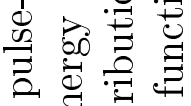

\&

I

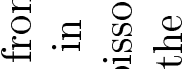

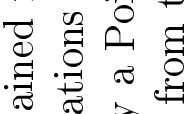

㐘要

0 要

ब

छ

䒕.

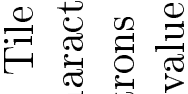

$\because$ 궝

ㄱ용

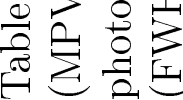


in just $(\sim 5 \%)$ of the events being rejected ${ }^{3}$.

\subsubsection{Background Considerations}

A possible source of background in this beam test could arise from the beam line: either by bremsstrahlung interactions of beam electrons as they travel through air ${ }^{4}$ and cross the ancillary scintillators ${ }^{5}$ or by any other type of soft particle that might come with the beam, or present in general in the experimental area. During a previous beam test of the ACD design concept in 2002 [191] at the same experimental line (H4) at SPS, a persistent level of background was observed after moving the calorimeter away from the beam and the ACD tiles. Such strategy cannot be applied in this beam test because all elements of the calibration unit are carefully integrated. Hence, another method should be employed to estimate the background contribution, if any.

Irrespective of the background intensity, the ansatz can be made that the observed probability distribution $P(x)$ is the sum of backsplash and background contributions:

$$
P(>x)=P_{\text {backsplash }}+P_{\text {background }}
$$

Since backsplash is due to secondary photons produced by the interaction of high energy particles in the calorimeter, the backsplash contribution is expected to correlate with the deposited energy. Therefore, in the limit where the deposited CAL energy is negligible,

$$
\lim _{C A L \text { Energy } \rightarrow 0} P(>x)=P_{\text {background }}
$$

\footnotetext{
${ }^{3}$ The ACD requirement regarding backsplash effect is to reject no more than $20 \%$ of otherwise accepted photons at $300 \mathrm{GeV}$.

${ }^{4}$ The beam setup was such that the beam particles moved through $\sim 2.5 \mathrm{~m}$ of air (i.e. $\lesssim 10^{-2}$ $X_{0}$ ) after exiting the low pressure beam pipe.

${ }^{5}$ About $\sim 2 \mathrm{~cm}\left(\sim 10^{-2} X_{0}\right)$ in total thickness for three scintillators (S0, S1, S2), as described in sec. 4.2 .3
} 


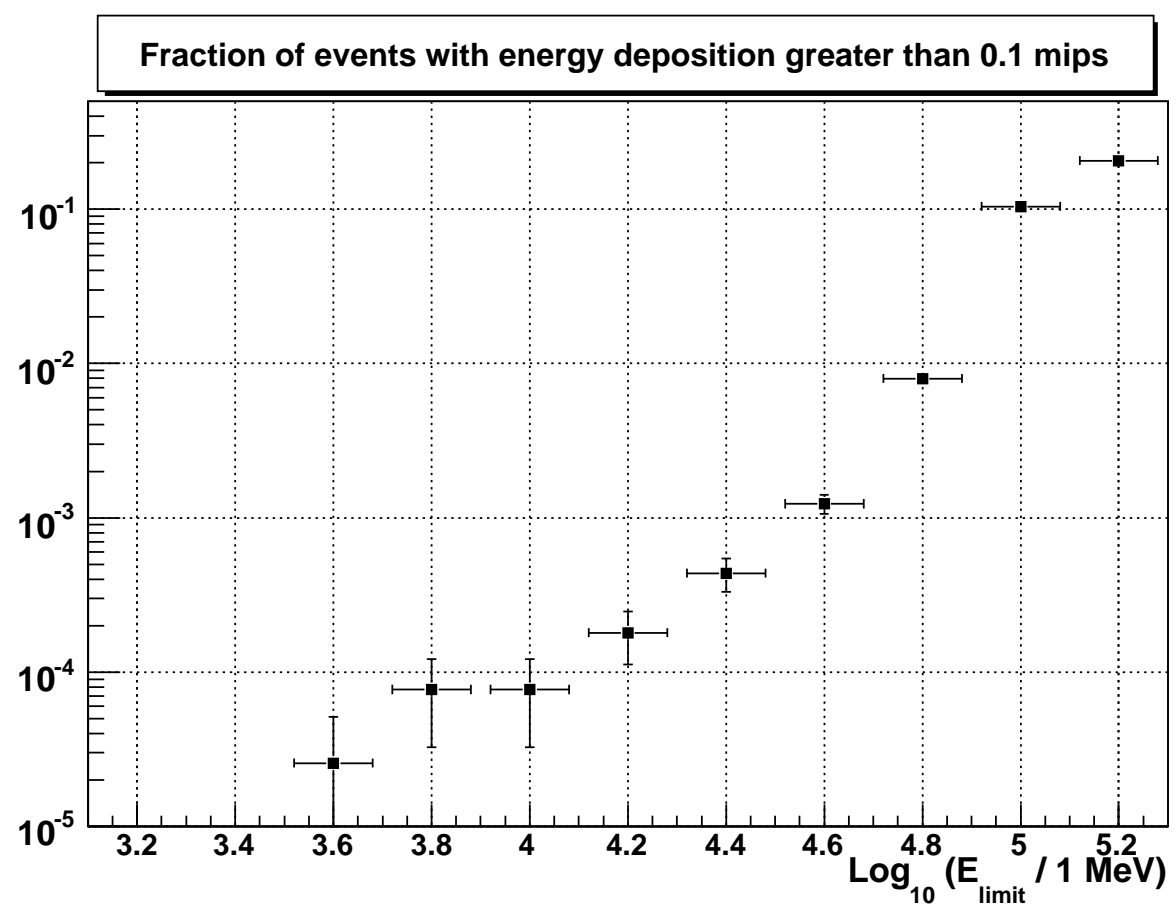

Figure 4.12: Fraction of events for which the signal in tile 110 is above 0.1 mips, as a function of the maximum energy in the CAL. Decrease in $E_{\text {limit }}$ is accompanied by decrease in tile occupancy, thus constraining the contribution of background contributions to backsplash.

This limit is extrapolated by considering the behavior of the ACD signals after successively decreasing -via event-energy selections- the maximum amount of energy deposited in the CAL $\left(E_{\text {limit }}\right)$. In the case of significant background intensity, there would a persistent signal that is independent of the energy measured in the calorimeter.

This is illustrated in fig. 4.12, where the tile occupancy above $0.1 \mathrm{mip}$ for tile 110 is shown as a function of $E_{\text {limit }}$. As the maximum amount of allowed energy in the calorimeter decreases, the signal occupancy in the tile is reduced to $\sim 10^{-4}$ which is a negligible amount when compared to the occupancy due to backsplash (fig. 4.11). This indicates that no significant level of background is present in the ACD signal after event selections. 


\subsubsection{Monte Carlo Simulations}

The same software framework used to analyze the LAT instrument has been adapted to study the beam test of the calibration unit. The simulation in this case combines a full description of the CU geometry (detectors and location) with the main properties of the beam (particle type, energy distribution, profile, angular dispersion etc.). The Monte Carlo software makes use of GEANT4 [91] to model the particle propagation through the instrument (bremsstrahlung, multiple-scattering, ionization, etc.) and the response of the detectors (energy depositions in silicon strips, CAL crystals and ACD tiles). As an illustration of the particle propagation and detectors response, a simulated event is shown in fig. 4.13. In particular, GEANT4 considers the creation of backsplash radiation by high energy particles incident in the instrument and follows this radiation as it propagates through the different detectors.

When a particle hits an ACD tile, the ACD response is calculated according to the following sequence of steps:

1. The energy deposited in the scintillator by each backsplash particle is calculated by GEANT4 taking into consideration the particle type, energy loss mechanisms, incident energy, and trajectory.

2. Correction due to non-uniform light collection at the edges is applied if required (when the incident particle hits the ACD tile close to the edge). As described above, the reduction factor by which the light collection decreases near the edge is different tile to tile, but in general, it is known from the LAT that the light collection is as bad as $\sim 70 \%$ at the tile edge, and recovers back to $100 \%$ when measured $\sim 3 \mathrm{~cm}$ away from the edge [190]. This represents an uncertainty in the analysis. As will be explained in the next section, two different scenarios will be considered in the simulation to bracket the expected backsplash probability.

3. The energy deposited in the tile (after edge correction) is converted to mip 


\begin{tabular}{|l|c|}
\hline \multicolumn{1}{|c|}{ Event Selection } & $\begin{array}{c}\text { Number of Events } \\
\text { Remaining }\end{array}$ \\
\hline \hline Total triggered events & 101157 \\
\hline Raw CAL energy $>1 \mathrm{GeV}$ & 100434 \\
\hline $\begin{array}{l}\text { TKR trigger within CU trigger window } \\
(\sim 600 \mathrm{~ns})\end{array}$ & 90163 \\
\hline $\begin{array}{l}\text { CAL Low trigger within CU trigger } \\
\text { window }(\sim 600 \mathrm{~ns})\end{array}$ & 83017 \\
\hline At least one reconstructed track & 82878 \\
\hline $\begin{array}{l}\text { Angle of best track with respect to beam } \\
<3^{\circ}\end{array}$ & 82021 \\
\hline$\chi^{2}$ of best track $<2.0$ & 74940 \\
\hline \multicolumn{1}{|c|}{ Remaining events after all cuts: } & 74940 \\
\hline
\end{tabular}

Table 4.5: Event selection cuts and number of events surviving each cut.

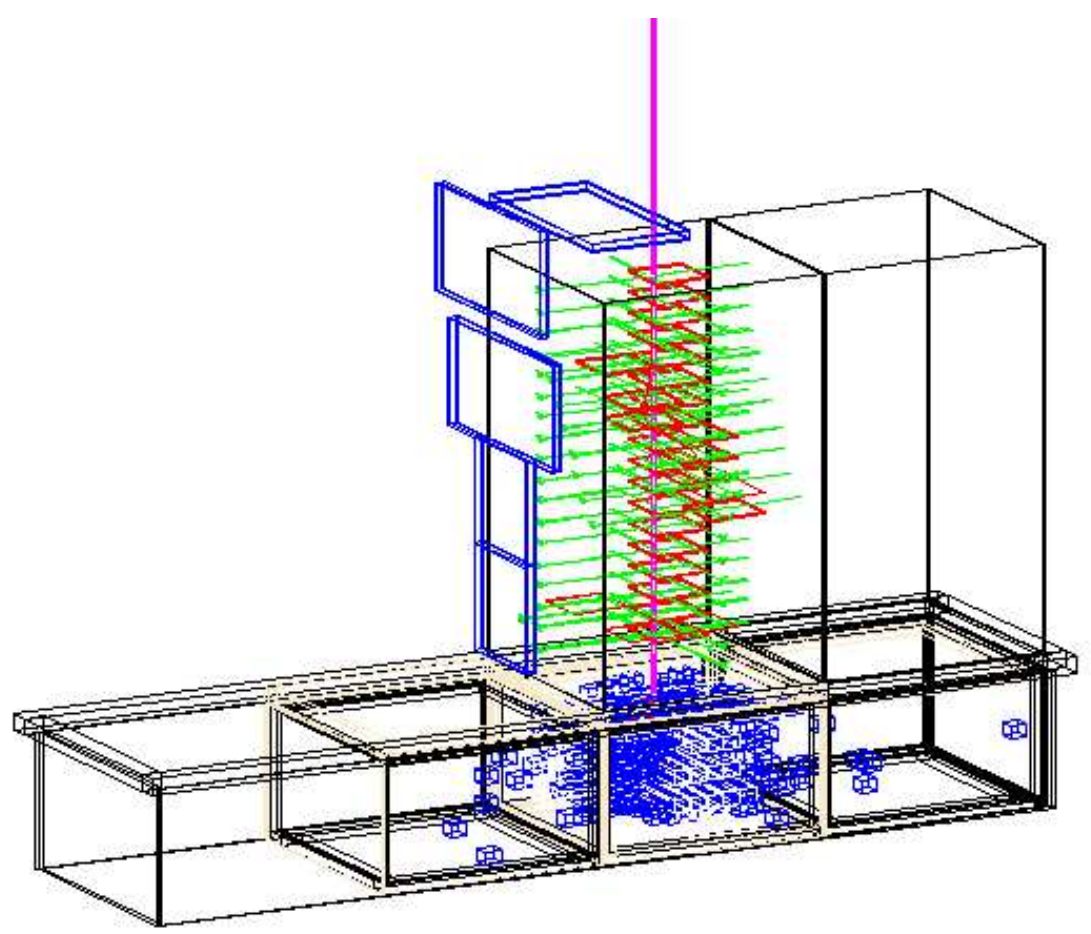

Figure 4.13: Event display of a $20 \mathrm{GeV}$ electron incident on the calibration unit. The electron trajectory is indicated by a purple line (angle of incidence of the event is $0^{\circ}$ and the impact point is at the center of the left tower). Hits in the TKR layers and CAL crystals can be seen in the figure. Neutral particles are not displayed for clarity. 
units, according to the relation $1 \mathrm{mip}=1.9 \mathrm{MeV}$.

4. For a given tile, the $N_{p e}$ value obtained during calibration is used to calculate the most likely number of photoelectrons produced when the collected light hits the PMT cathode. Poisson fluctuations are applied to this number and its subsequent gains in the PMT first few stages (dynodes). Fluctuations thereafter have negligible impact on the overall statistics and consequently are not implemented in the simulation.

5. In the last step a small Gaussian fluctuation is applied to the ACD signal to simulate electronics noise. The variance of this fluctuation is estimated from the pedestal width observed during ACD calibration, which is about $\sim 3 \times 10^{-3}$ mips on average for all the tiles.

\subsubsection{Comparison of Data and Monte Carlo Simulations}

Figures 4.14-4.17 show the obtained backsplash distributions for all the tiles as obtained from the beam test data (black points) for a $200 \mathrm{GeV}$ electron beam with statistical errors. Each figure includes the expected backsplash distribution expected from Monte Carlo simulations. As explained before, the main uncertainty in the simulation is the light collection uniformity at the edge of every tile. Thus, the expected backsplash distribution is bracketed by two extreme scenarios:

- In the minimum light collection efficiency scenario, edge corrections are applied within $3 \mathrm{~cm}$ of the tile edge. The light collection efficiency is assumed to decrease linearly from $100 \%$ to $70 \%$ within this edge.

- In the maximum light collection efficiency scenario, no edge corrections are applied. 
Both scenarios are shown in the backsplash figures as "bands" that bracket the expected backsplash distribution. The width of each band is equivalent to twice the statistical error $(2 \sigma)$ obtained from the simulation.

As can be seen from the figures, the Monte Carlo simulations are able to reproduce very well the backsplash distribution. The only significant difference between data and simulation is found for the lowest energy deposition $(\sim 0.1$ mips; first bin in the plot) for tiles $0,110,120$. It should be noted however, that these differences between Monte Carlo and data are at the $\sim 3 \%$ level or less. Possible reasons for the small remaining discrepancies include differences between the beam and its Monte Carlo representation, such as beam profile, beam angular dispersion, impact position and incident angle.

\subsubsection{Backsplash Energy Dependence}

The measured backsplash energy dependence observed in tile 110 is shown in fig. 4.18. As expected, the backsplash probability correlates with the beam energy. Furthermore, it can be seen that backsplash does not increase dramatically at the highest energies. This is due to the fact that the shower is not fully developed and contained in the calorimeter at the higher energies, and thus, the raw energy deposited in the calorimeter by the electromagnetic shower does not scale linearly with the event energy $^{6}$.

An empirical formula was found in the beam test of the ACD design choices in 2002 to describe the backsplash probability [191]. The energy dependence of such formula is given by,

$$
P_{b a c k s p l a s h} \propto \sqrt{E}
$$

and as can be seen in fig. 4.18, it fits well the data and thus corroborates the results

\footnotetext{
${ }^{6}$ As discussed in Chapter 3, the segmented calorimeter provides a clear image of the shower profile, which is used to calculate the actual energy of the event.
} 


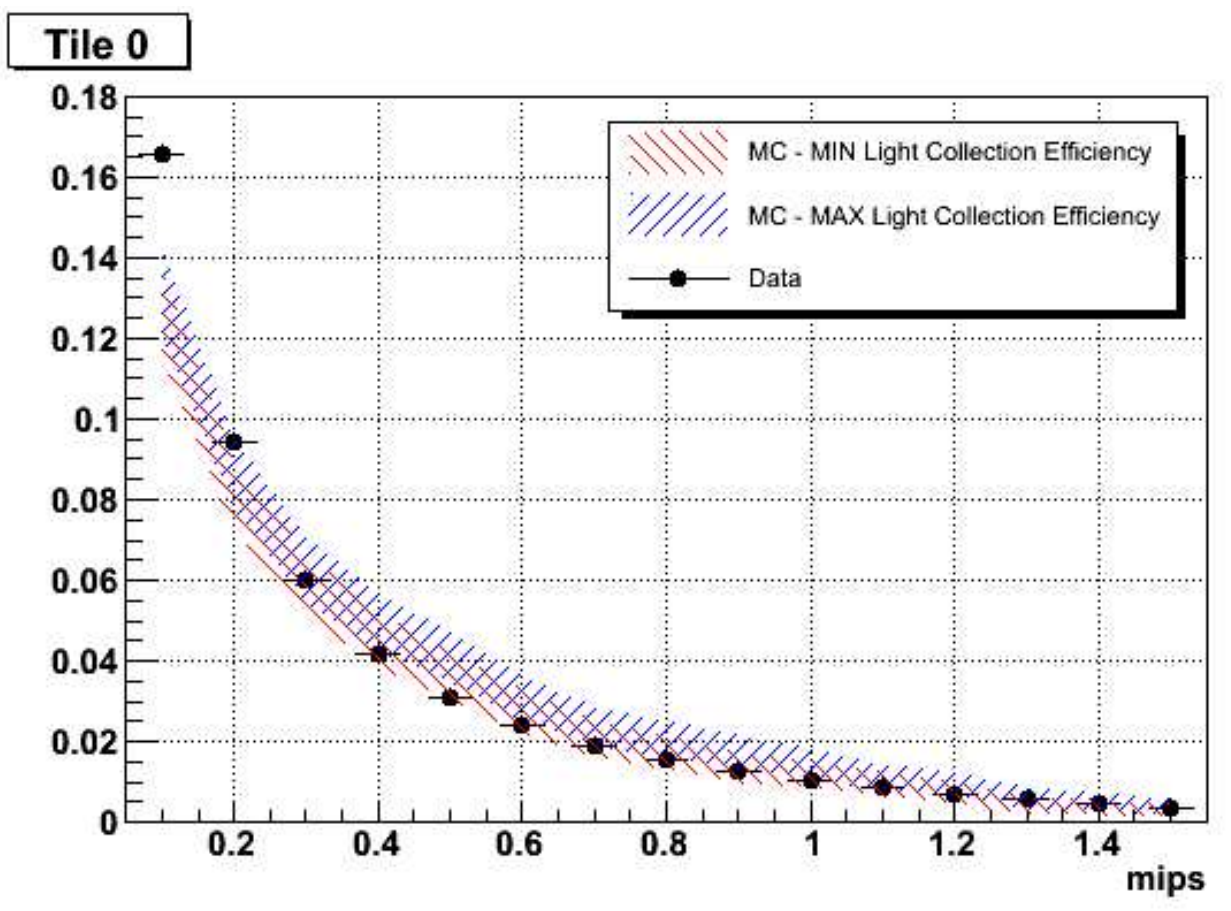

Figure 4.14: Backsplash distribution for tile 0 as obtained from beam test data (black points) and Monte Carlo expectations. In every case, backsplash is expressed as the fraction of events for which the signal in the tile is above a given threshold. The error bars in the data are statistical $(1 \sigma)$. Monte Carlo simulations consider two extreme scenarios for light collection uniformity at the tile edge. In the MIN efficiency scenario, the collection efficiency decreases linearly from $100 \%$ (3 cm away from the edge) to $70 \%$ at the tile edge. In the MAX case, the collection efficiency is $100 \%$ throughout the tile edge. The width of each Monte Carlo band is statistical $(2 \sigma)$. 


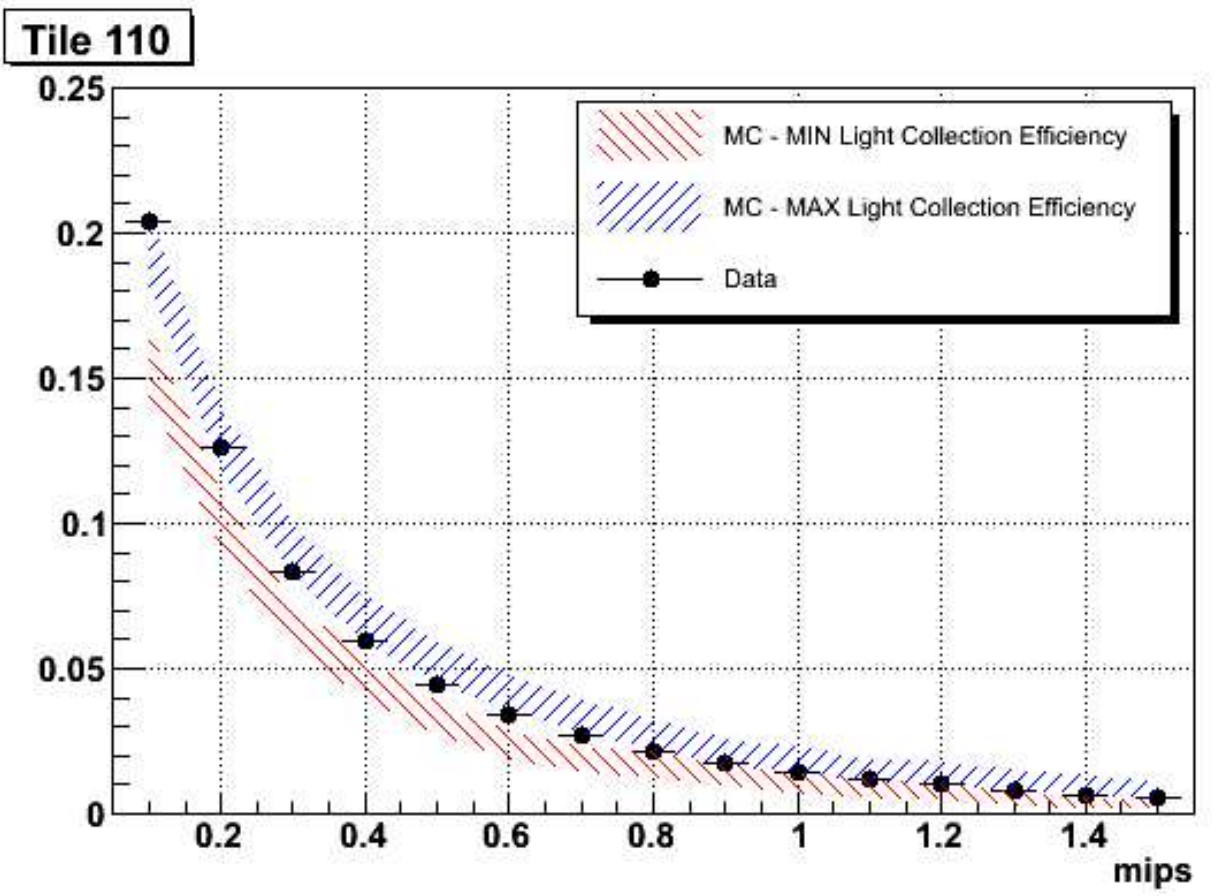

Figure 4.15: Backsplash distribution for tile 110 as obtained from beam test data (black points) and Monte Carlo expectations. In every case, backsplash is expressed as the fraction of events for which the signal in the tile is above a given threshold. The error bars in the data are statistical $(1 \sigma)$. The description of the Monte Carlo data can be found in the caption of fig. 4.14. 


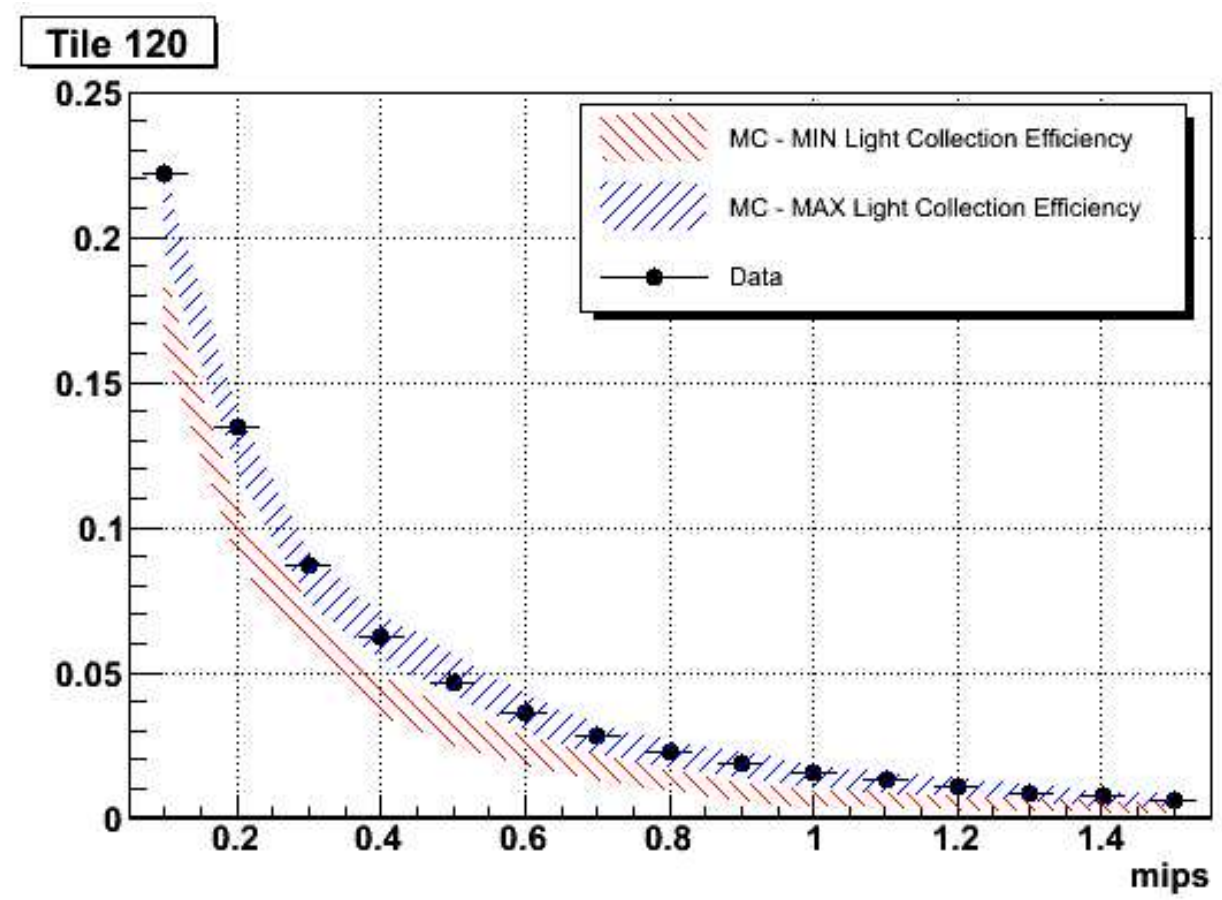

Figure 4.16: Backsplash distribution for tile 120 as obtained from beam test data (black points) and Monte Carlo expectations. In every case, backsplash is expressed as the fraction of events for which the signal in the tile is above a given threshold. The error bars in the data are statistical $(1 \sigma)$. The description of the Monte Carlo data can be found in the caption of fig. 4.14. 


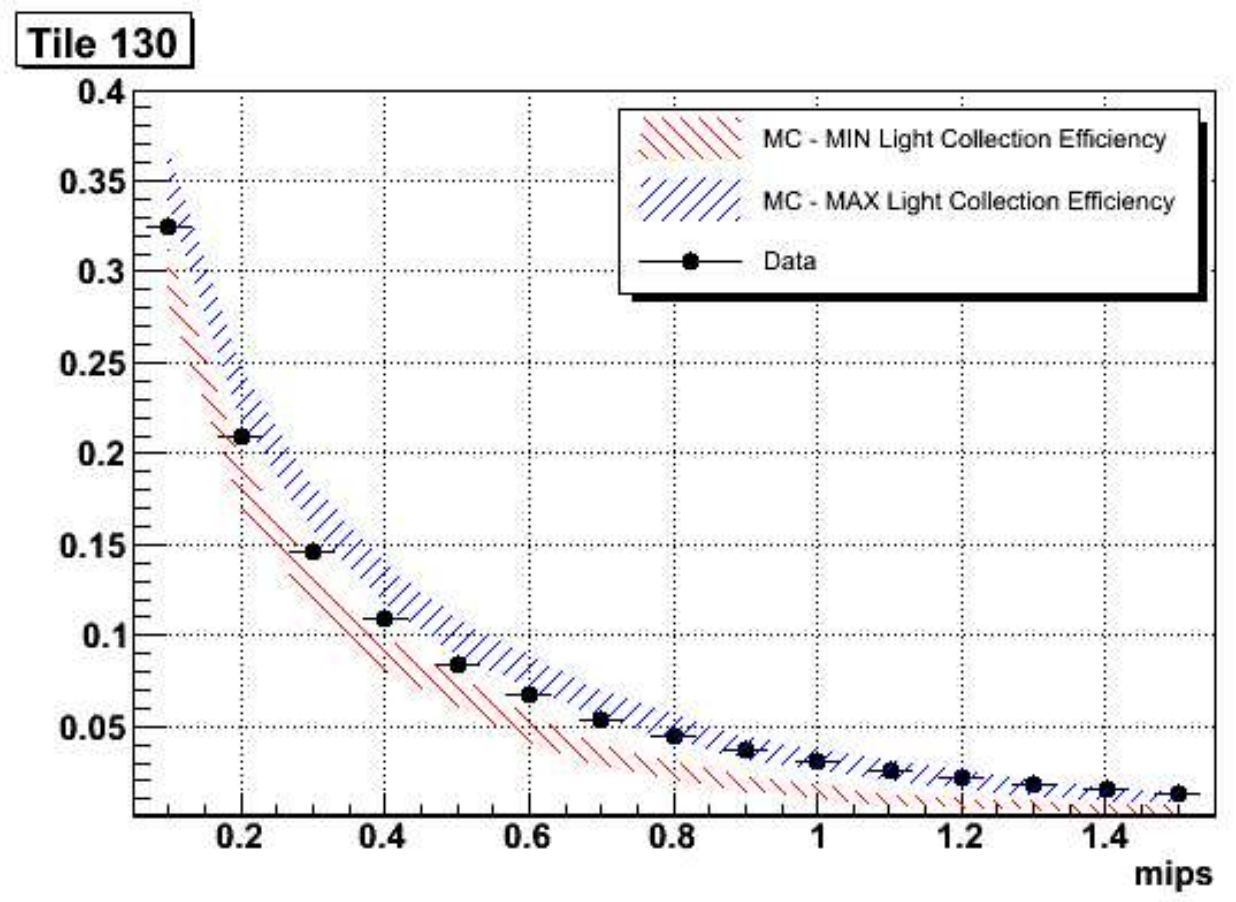

Figure 4.17: Backsplash distribution for tile 130 as obtained from beam test data (black points) and Monte Carlo expectations. In every case, backsplash is expressed as the fraction of events for which the signal in the tile is above a given threshold. The error bars in the data are statistical $(1 \sigma)$. The description of the Monte Carlo data can be found in the caption of fig. 4.14. 


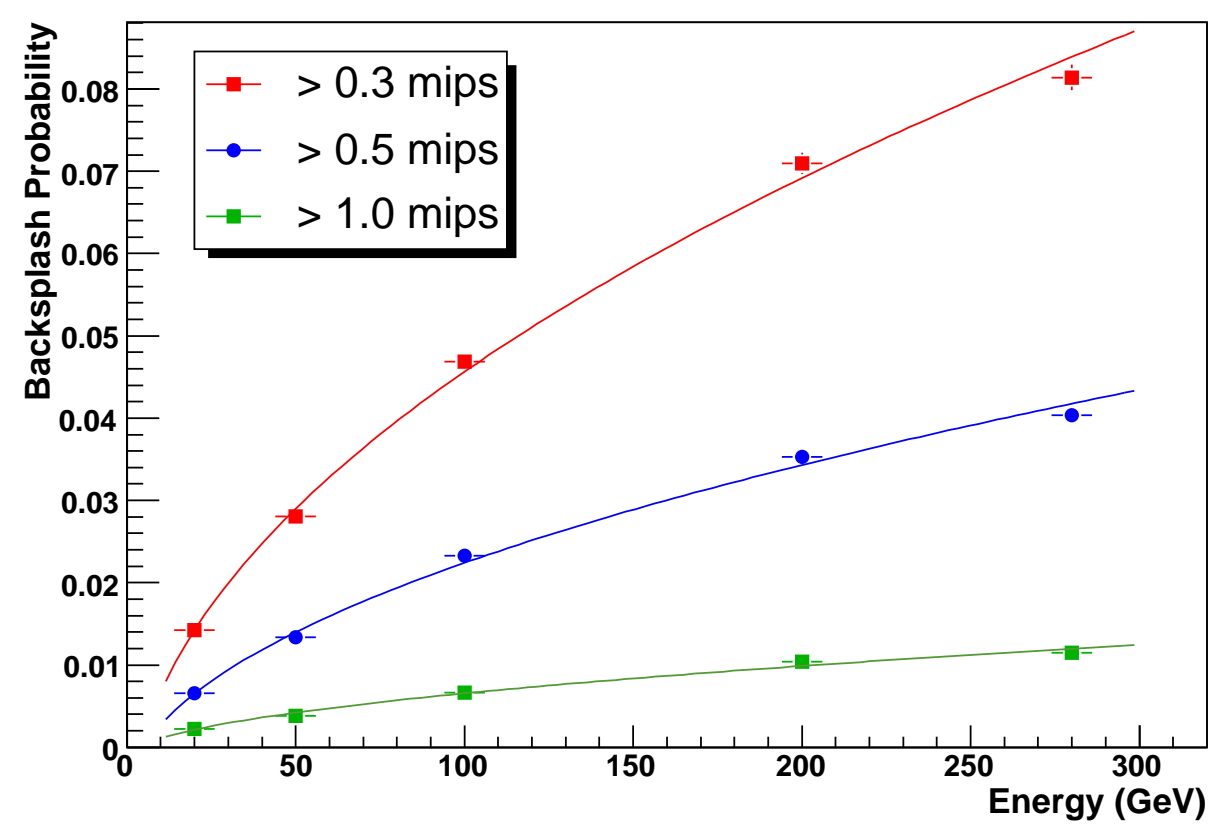

Figure 4.18: Energy dependence of backsplash in tile 110 for different thresholds. The data is well fitted by a function of the form $\sqrt{E}$.

obtained in that beam test.

\subsection{Summary and Conclusions}

The LAT effective area at high energies (which is fundamental for EBL studies) must be preserved by minimizing the fraction of high energy $\gamma$-rays that are rejected due to backsplash self-veto. This could be achieved by increasing the veto threshold, as can be gathered from fig. 4.18. However, there is a limit to which this can be done, since the veto threshold must be kept low enough to ensure a highly efficient charged particle detection, which is critical for on-orbit background rejection. These two competing effects must be reconciled, and ACD segmentation makes it possible. As measured during this beam test, the backsplash probability for individual tiles at a veto threshold of 0.45 mips ranges from $\sim 3 \%$ to $\sim 10 \%$, depending on the tile location and size. Thus, the probability that a high energy $\gamma$-ray will create a backsplash 


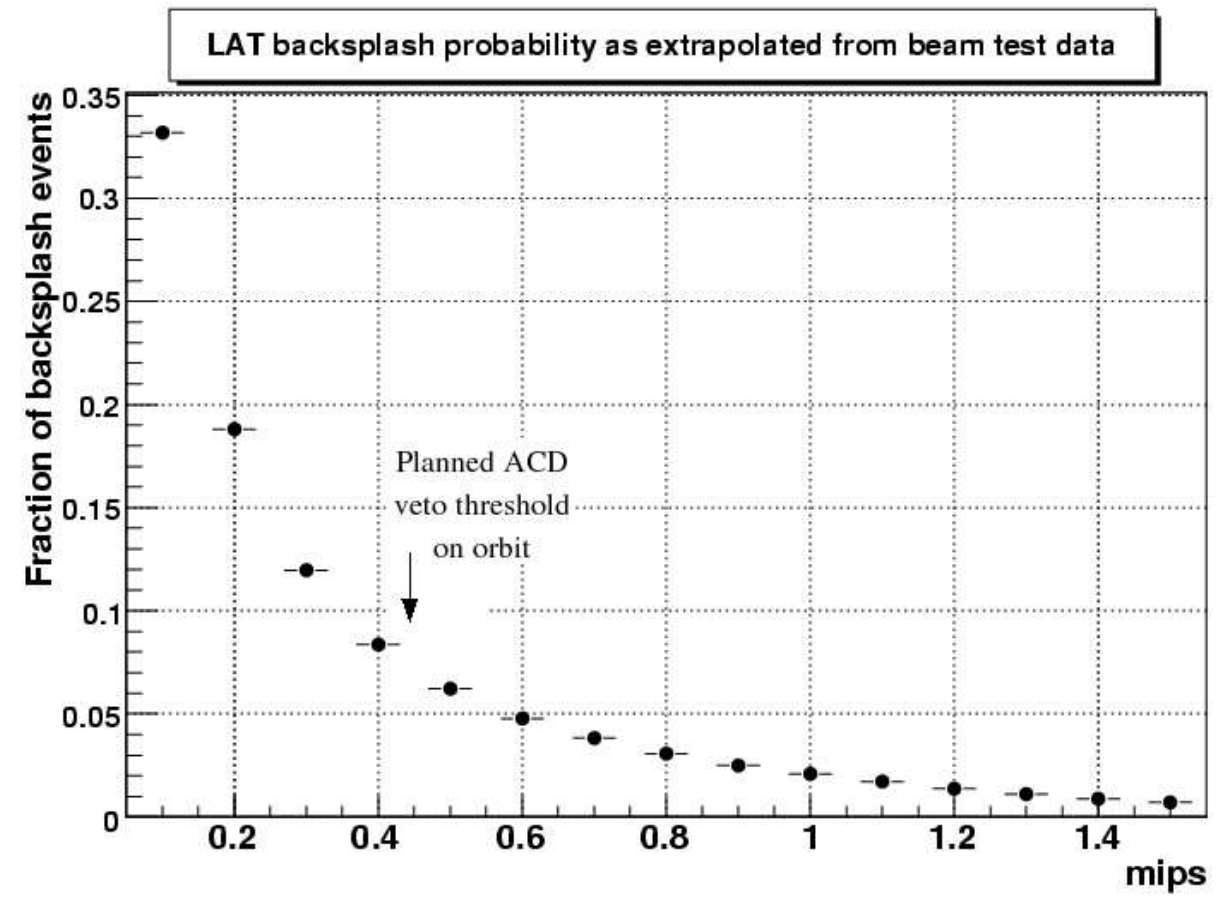

Figure 4.19: Estimated LAT ACD backsplash probability (top tile, for a normally incident $280 \mathrm{GeV}$ electron), as extrapolated from the beam test data. As indicated in the plot, for a veto threshold of 0.45 mips the backsplash self-veto probability is about $\sim 10 \%$. 
signal in the particular ACD tile that it crossed can be constrained with the level of segmentation in the ACD design.

The LAT ACD was designed with the requirement that backsplash self-veto should remove not more than $20 \%$ of otherwise accepted $\gamma$-ray events at $300 \mathrm{GeV}$ [191]. The compliance of the design to this requirement has been tested with Monte Carlo simulations, that with this analysis, have been empirically verified (for the first time with actual flight-like hardware and data acquisition electronics).

In particular, tile 0 can be used to estimate the backsplash probability of a single ACD tile located at the top of the LAT, when crossed by a high energy $\gamma$-ray. It should be noted that the distance between this tile and the calorimeter is very similar (within a few centimeters) to the corresponding distance on the LAT. The only difference between tile 0 in the calibration unit and an actual LAT ACD tile is the area: $\sim 550$ $\mathrm{cm}^{2}$ for the former and $\sim 1100 \mathrm{~cm}^{2}$ for the latter. Thus, the backsplash probability in a top LAT-ACD tile is roughly twice the value obtained in this beam test, as illustrated in fig. 4.19 for a $280 \mathrm{GeV}$ electron at normal incidence. 


\section{Chapter 5}

\section{Detecting the EBL Attenuation of Blazars with GLAST}

\subsection{The Impact of GLAST on AGN Science}

GLAST will bring important advances to the study of blazars and AGN thanks to the improvements in instrument performance (PSF, effective area, field of view, energy range and energy resolution) described in Chapter 3. Directly related to the topic of this dissertation, the most important expected advances that GLAST will make possible are i) a significant increase, by at least one order of magnitude, in the number of identified blazars up to very high redshifts $(\mathrm{z} \sim 5)$, and ii) precise measurements of the spectra of these blazars over the broad LAT energy range $(20 \mathrm{MeV}$ to $>300 \mathrm{GeV})$.

Although not all the advances during the GLAST era are likely to have a direct impact in the study of the EBL, it is still worthwhile to give a brief but general overview of the areas where GLAST observations are expected to bring significant progress in the understanding of blazars (Science Goals document [166] from the LAT's Blazar and other $A G N$ science group):

- Physics of $\gamma$-ray emission in blazars and properties of radio-loud AGN 
in general. This relates to the physical processes by which radio-loud AGN accrete material, accelerate particles and radiate (up to $\gamma$-ray energies). GLAST observations will be especially useful to understand the formation, structure and content of jets, where particle acceleration takes place, and the resulting radiative processes.

- Blazar Spectra: Observations with the improved sensitivity and energy resolution of GLAST, in conjunction with other multi-wavelength observations, will reveal the spectra of blazars over a wide energy range. These broadband observations will be combined with theoretical models to test and develop a consistent picture of $\gamma$-ray emission process.

- Blazar Variability: The increased effective area of GLAST, combined with its large FOV and all-sky coverage ${ }^{1}$ will allow for constant monitoring of the $\gamma$-ray flux and spectra over a wide range of timescales, during flaring and quiescence states, providing thus an overall view of the dynamical behavior of blazars.

- Blazars as a population. Given the instrument's point source sensitivity (sec. 3.6.3), the number of blazars detected by GLAST will range from a thousand [56] to several thousand [43, 195, 257]. A sample of sources so large will allow for a detailed study of the luminosity distribution of blazars and its different subclasses (FSRQs, BL Lacs). As described in [188], GLAST observations will probe the evolutionary connection between FSRQs and BL Lacs, verify the unified model for radio-loud AGN [270], and test (or even reformulate) the "blazar sequence" [86].

\footnotetext{
${ }^{1}$ The default observing mode of GLAST is the survey or scanning mode. In this mode, the spacecraft slews once per orbit, alternating between 35 degrees above and 35 degrees below the orbit plane. This provides observation of the full sky every two orbits ( 190 mins). The primary goals of survey mode are to provide uniform sky coverage and to maximize GLAST's ability to study the behavior of time variable sources like blazars over a wide range of timescales.
} 
- Blazars as an astrophysical tool. Deeper understanding of blazars will make them useful probes of i) the matter content of the region around the black hole, and ii) the radiation content of the universe (EBL). The second item is the topic of this dissertation and will be discussed in detail below.

This chapter introduces two complementary analyses to detect and measure the EBL attenuation of blazars with GLAST observations. Each technique will be demonstrated with Monte Carlo simulations that combine established models of the expected blazar luminosity function at gamma-ray energies, different models of the opacity of the universe to $\gamma$-rays due to EBL absorption, and GLAST's instrument performance. The results obtained with each technique prove that gamma-ray blazars have the potential to be a highly effective probe of the optical-UV EBL and its evolution over cosmic time. The organization of the chapter is as follows: first, the luminosity function of blazars is introduced to provide reasonable estimates for the number of blazars that GLAST will detect. Thereafter, the two analysis techniques, namely, the flux ratio and spectrum fitting are described and tested. Other issues, like the possibility of "cosmic conspiracies" and the importance of redshift determination for GLAST-detected blazars are discussed at the end of the chapter.

\subsubsection{Blazar Luminosity Function}

For a population of discrete astrophysical sources like blazars, the luminosity function describes the distribution of the objects as a function of their luminosity and redshift. In general, the number of sources with luminosity $L$ in a volume element $d V$ at redshift $z$ can be expressed in terms of the luminosity function $\phi(L, z)$ :

$$
d N(L, z)=\phi(L, z) d V(z) d L
$$


To determine the luminosity function from observations, one needs a collection of objects having at least flux and redshift information. Then, using the redshift as a distance indicator, the flux-number surface density (better known as $\log (N)-\log (S)$ plot) can be unfolded to give the number of sources per unit volume as a function of blazar luminosity, which is the fundamental quantity. It should be noted that the redshift of a blazar cannot be determined from $\gamma$-ray observations alone due to the lack of "line" features in the gamma-ray spectra of blazars. Redshift determination must be accomplished at longer wavelengths (infrared to UV), just another example of the importance of multi-wavelength observations.

The number density of AGNs in general (and of blazars in particular) is a fundamental piece of cosmological information since it provides a measure of the time scale for supermassive black hole formation in the early universe. Unfortunately, the available data is often incomplete or biased, especially at fluxes close to the detection threshold; making necessary to use different techniques, assumptions and models to overcome the incompleteness of the sample. By extending the $\log (N)-\log (S)$ curve to fluxes about 25 times lower than EGRET, GLAST observations will mark a milestone in the understanding of blazars and their luminosity function, with the condition that redshifts for the individual sources are obtained in a joint effort with the astronomical community.

EGRET established blazars as the dominant class of extragalactic $\gamma$-ray emitters with the high confidence detection of more than 60 sources, most of them FSRQs and a few BL Lacs. The $\log (N)-\log (S)$ in figure 5.1 presents the flux distribution observed by EGRET (solid lines) and different model predictions (dashed, dot-dashed lines) that extrapolate the luminosity function of blazars down to fainter fluxes. Given the point source sensitivity for $5 \sigma$ detection of GLAST (one-year long, sky-survey mode), the models predict that the number of blazars to be detected by GLAST ranges from 〜1000 (Dermer ; short-dashed lines) to 10000 (Stecker \& Salamon, long-dashed 


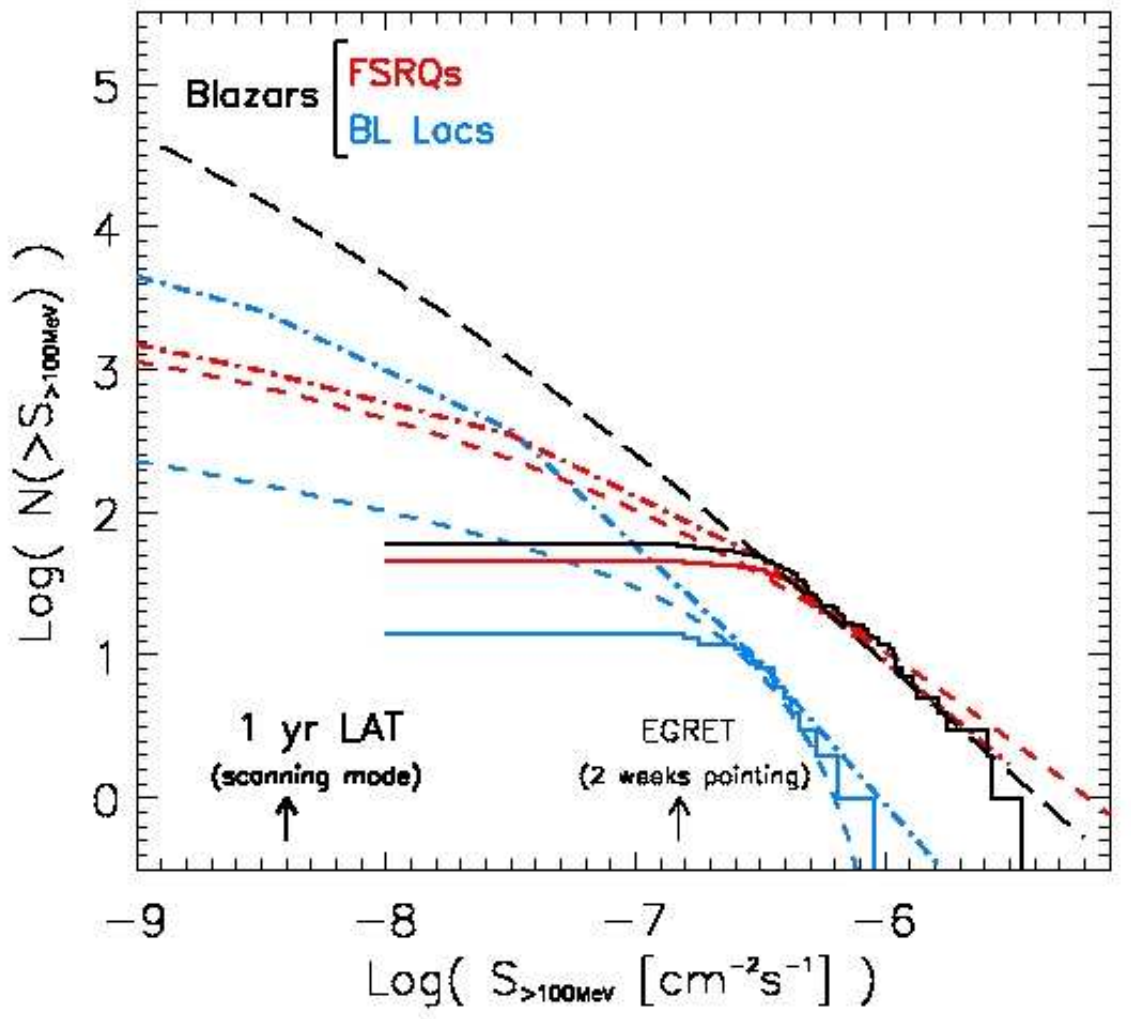

Figure 5.1: Blazar $\log (N)-\log (S)$ curve from [188]. The plot contains the distribution observed by EGRET (solid lines) and various model predictions (dashed and dot-dashed lines), which eventually will be replaced with GLAST data. The source counts from the EGRET data and some of the model predictions are further subdivided into FSRQs (red lines) and BL Lacs (blue lines) contributions. The included model predictions are: Stecker \& Salamon (black dashed line ; [255]), Mücke \& Pohl (dot-dashed line ; [195]), and Dermer (short-dashed lines ; [56]). The sensitivities of EGRET (two-week long, pointed observations) and GLAST (one year, scanning mode) are indicated. The predicted number of blazars to be observed by GLAST ranges from $\sim 1000$ (Dermer) to $\sim 10000$ (Stecker \& Salamon). 
line). This would increase the number of known $\gamma$-ray blazars (in either scenario) by at least one order of magnitude ${ }^{2}$. It is important to note, however, that these models are predictions to be tested in the near future by GLAST, and that eventually, the $\log (N)-\log (S)$ plot will be populated with actual data.

The blazar luminosity function affects the extent to which blazars can be used to probe the EBL. Indeed, the techniques proposed in this dissertation to detect and measure the EBL attenuation of blazars were designed specifically to take advantage of the large number of blazars that GLAST is expected to observe.

\subsection{Flux-Ratio Method}

The major challenge in the study of the EBL attenuation of blazars is to distinguish the attenuation due to extragalactic absorption from intrinsic peculiarities that could be present in the spectrum of the individual sources. The flux-ratio method ${ }^{3}$ offers a powerful way to make this distinction through a statistical analysis of a large sample of blazars, by measuring the common level of EBL attenuation experienced by sources located at similar redshifts.

\subsubsection{Monte Carlo Simulation}

A Monte Carlo simulation is used to demonstrate the flux-ratio technique. The simulation combines established models of the blazar luminosity function at $\gamma$-ray energies, the opacity due to EBL absorption according to different models introduced in Chapter 2, and GLAST's instrument performance.

\footnotetext{
${ }^{2}$ For comparison, the number of high confidence detected blazars at other wavelengths is about 1000, with over 7000 blazar candidates. Blazar catalogs are described in sec. 5.4.2.1

${ }^{3}$ Published by Andrew Chen, Steve Ritz and the author himself [42].
} 


\subsubsection{Simulated Blazar Populations}

Since the blazar luminosity function determines the statistical power of the technique, and since this function is still unconstrained down to the GLAST sensitivity, two different models for the luminosity function are used to obtain reasonable estimates of the number of blazars that GLAST will detect and its redshift distribution. These two models are briefly discussed below.

\section{Stecker \& Salamon Luminosity Function}

The first model, by Stecker and Salamon [255], assumes that blazars and FSRQs are the same population. Not all FSRQs are observed as $\gamma$-ray blazars because the gamma-ray radiation is narrowly beamed, and accordingly, only a small fraction of the sources are pointing at Earth. An additional assumption is that the radio and gammaray luminosities are linearly related $L_{\gamma}=\kappa L_{r}$. The number density of gamma-ray blazars $\rho_{\gamma}$ and FSRQs $\rho_{r}$ is given then by,

$$
\begin{aligned}
\rho_{\gamma}\left(L_{\gamma}, z\right) & =\eta \rho_{r}\left(L_{r}, z\right) \\
& =\eta \rho_{r}\left(\frac{L_{\gamma}}{\kappa}, z\right)
\end{aligned}
$$

where $\kappa$ is a proportionality constant and $\eta=\left(\theta_{\gamma} / \theta_{r}\right)^{2}$ is a parameter of the model that takes into account the reduction in the number of blazars, as compared to FSRQs, because of the narrow beaming angle for gamma-ray emission $\theta_{\gamma}$ (as compared to $\theta_{r}$ ). Since the FSRQ radio luminosity function is known from radio observations [66],

$$
\rho_{r}\left(L_{r}, z\right)=10^{-8.15}\left\{\left[\frac{L_{r}}{L_{c}(z)}\right]^{0.83}+\left[\frac{L_{r}}{L_{c}(z)}\right]^{1.96}\right\}^{-1}
$$

with $\log _{10} L_{c}(z)=25.26+1.18 z-0.28 z^{2}$, the gamma-ray luminosity function for blazars is straightforward to calculate, 


$$
\rho_{\gamma}\left(L_{\gamma}, z\right)=\eta \times 10^{-8.15}\left\{\left[\frac{L_{r} / \kappa}{L_{c}(z)}\right]^{0.83}+\left[\frac{L_{r} / \kappa}{L_{c}(z)}\right]^{1.96}\right\}^{-1}
$$

The units of comoving density $\rho$ are $\mathrm{Mpc}^{-3} \mathrm{x}$ (unit interval of $\left.\log _{10} L\right)^{-1}$ and the units of $L$ are $\mathrm{W} \mathrm{Hz}^{-1} \mathrm{sr}^{-1}$. Stecker \& Salamon constrained their model to predict the number of blazars detected by EGRET, given its instrumental flux sensitivity. In their original paper, Stecker \& Salamon used cosmological parameters $\Omega_{M}=1$, $\Omega_{\Lambda}=0$, and $H_{0}=50 \mathrm{~km} \mathrm{~s}^{-1} \mathrm{Mpc}^{-1}$, which are in contradiction with the current values, as measured by WMAP (Wilkinson Microwave Anisotropy Probe [250, 249]). The impact of this on the number of detected blazars is minimum since the small bias introduced when calculating the luminosity function is self-corrected when calculating the flux (by using a the same set of cosmological parameters). The original values from the model are thus preserved here for simplicity.

Given the blazar's redshift $z$ and differential luminosity $L_{\gamma}(E)$, the observed integral flux above some energy $\mathrm{E}_{0}$ can be calculated as ${ }^{4}$

$$
F\left(E_{0}\right)=\frac{L_{\gamma}\left(E_{0}\right)}{\alpha(1+z)^{\alpha+1} r^{2} R_{0}^{2}}
$$

where $\alpha$ is the spectral index for the blazar and $r R_{0}$ is a measure of cosmological distance given by $r R_{0}=\frac{2 c}{H_{0}}\left(1-(1+z)^{-1 / 2}\right)$.

Using this, Stecker \& Salamon predict the number of sources at redshift $z$ observed at Earth with an integral flux $F$

$$
\frac{d N}{d F d z} \Delta z \Delta F=4 \pi r^{2} R_{0}^{3} \times \Delta r \times \rho\left(L_{\gamma}, z\right) \times \Delta\left(\log _{10} L_{\gamma}\right)
$$

\footnotetext{
${ }^{4} \mathrm{~A}$ factor of $4 \pi$ is absent from the equation because $L_{\gamma}$ is the differential luminosity per steradian
} 


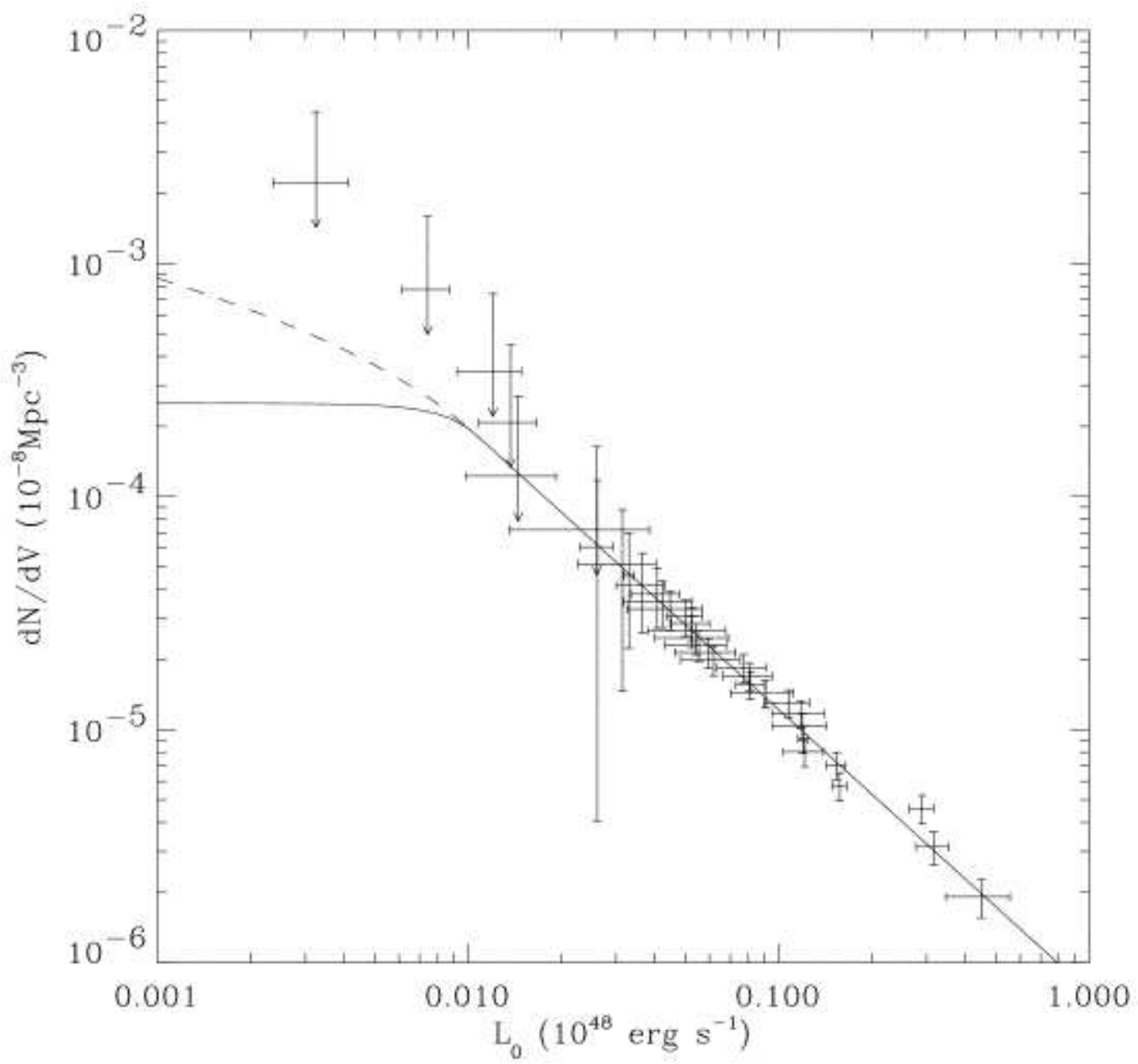

Figure 5.2: From [43]. Cumulative luminosity function derived from the de-evolved luminosities by Chiang \& Mukherjee. The solid line is a broken power law that has been fitted to the de-evolved luminosities for $L_{0}>10^{46} \mathrm{erg} \mathrm{s}^{-1}$ and to the redshift distribution at the low luminosity end. The dashed line is the $99 \%$ confidence limit on the low end of the fit. 


\section{Chiang \& Mukherjee Luminosity Function}

The model by Chiang \& Mukherjee [43] does not assume a correlation between the blazar luminosity at gamma-ray energies and the luminosity at any other band. Instead they pursue a unified picture based on all the blazars detected by EGRET. Any possible bias resulting from missing redshifts is avoided by requiring every source to be included in the $1 \mathrm{Jy}$ catalog ( 1 Jansky $=10^{-26} \mathrm{~W} \mathrm{~m}^{-2} \mathrm{~Hz}^{-1}$ ) of radio sources of Kuhr et al [149] (all of the EGRET sources contained in this catalog have optical identifications and measured redshifts).

This model tries to address directly the evolution of blazars. Although there is mounting evidence that the blazar population evolves with cosmic time [17, 154, 171], there is still not clear understanding on how the individual blazars change with time. There are two extreme possibilities: either a constant but small fraction of galaxies harbor radio-loud AGN, and the luminosities of these sources change systematically with time ("luminosity evolution"), or most galaxies harbor AGN, but at any given time most are in "quiet" states. In the latter case, the fraction of galaxies with radio-loud AGN in an active state changes with time ("density evolution"). Chiang \& Mukherjee consider separately both scenarios. Pure density evolution is considered by parameterizing the luminosity function; both as an exponential function $\exp \left[\beta \tau(z) H_{0}\right]$, and as a power law $(1+z)^{\beta}$. $\beta$, the "evolution parameter" is then determined by applying the method of maximum likelihood to the data. No evidence of pure density evolution was found ${ }^{5}$.

Next, pure luminosity evolution is considered through several functional forms, with the power law $\left(f(z)=(1+z)^{\beta} ; \beta=2.7\right)$ being the preferred one after the respective fits and statistical tests are performed. With the evolution already determined, the cumulative luminosity function is calculated (see fig. 5.2). Focusing

\footnotetext{
${ }^{5} \mathrm{~A}$ recent analysis by Narumoto \& Totani [200] finds evidence of luminosity-dependent density evolution, which is the favored model for the X-ray luminosity function of AGN. This could indicate that the jet activity is universally correlated with the accretion history of AGNs.
} 


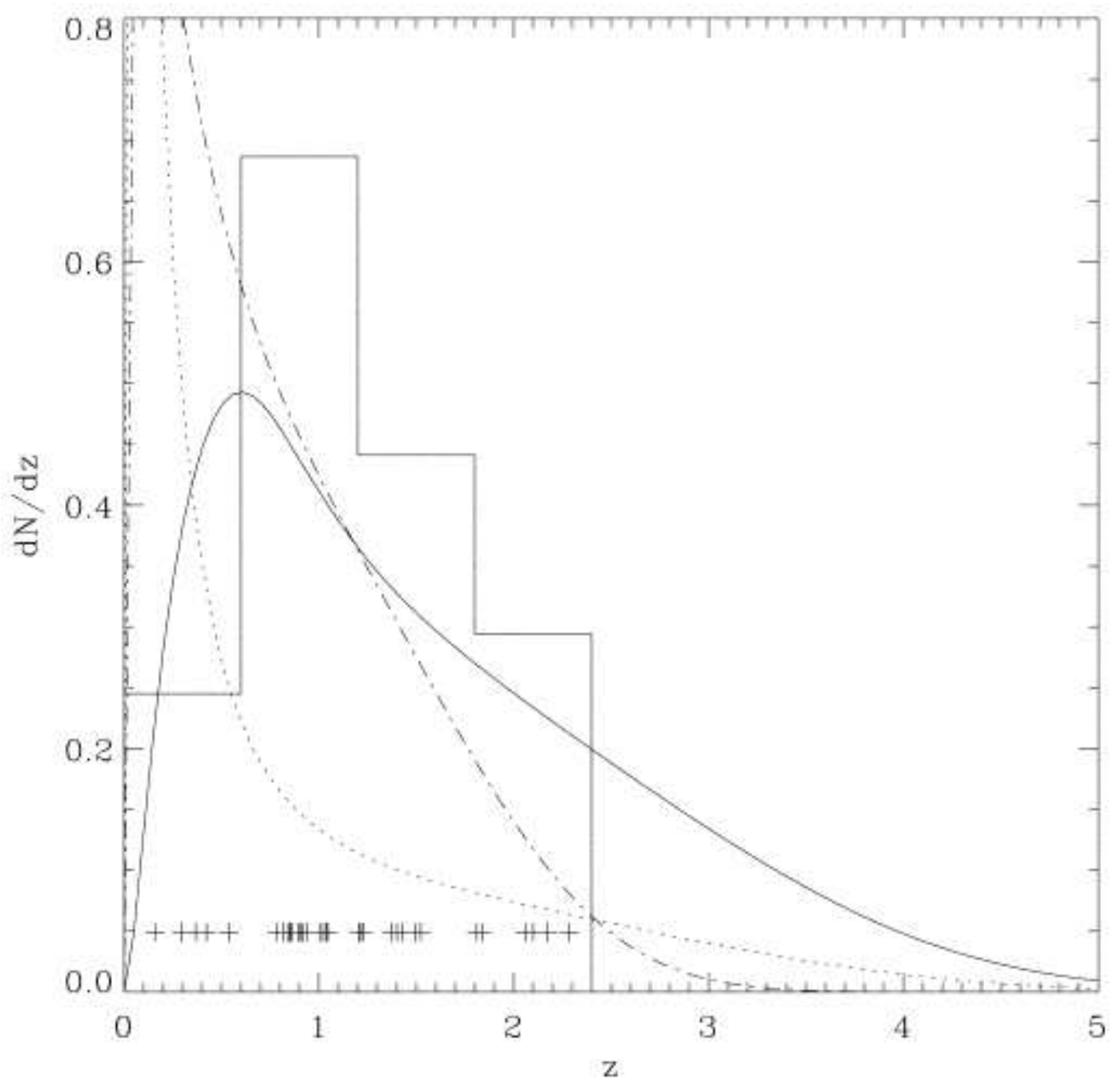

Figure 5.3: From [43]. Redshift distribution of EGRET sources and expected redshift distribution according to the model by Chiang \& Mukherjee. Crosses and histograms are EGRET data. The dotted curve is the model redshift distribution using a single power law fitted to the high luminosity end of Fig.5.2 with cutoff at the minimum measured luminosity in the sample. The solid line is obtained by fitting to a broken power law (adopted method). The dot-dashed curve is the redshift distribution from Stecker \& Salamon luminosity function discussed above. 
initially just in the data points, it is clear that the distribution is well constrained only in the high luminosity end $\left(L_{0}>10^{46} \mathrm{erg} \mathrm{s}^{-1}\right)$.

Nevertheless, additional information is available in the redshift distribution. This can be used to constrain the low luminosity end of the luminosity function. A single power law does not fit well the data since such model fails to reproduce the actual redshift distribution of the EGRET sources reproduced in fig. 5.3 (dotted line). Instead, a broken power law can be used to fit the data, with the redshift distribution of the EGRET blazars being used to determine the break luminosity and the index of the lower end of the luminosity function.

The resulting luminosity function is parametrized as

$$
\begin{aligned}
\frac{d N}{d L_{0} d V} \propto\left(\frac{L_{0}}{L_{B}}\right)^{-\gamma_{1}}, & L_{0} \leq L_{B} \\
\frac{d N}{d L_{0} d V} \propto\left(\frac{L_{0}}{L_{B}}\right)^{-2.2}, & L_{0}>L_{B}
\end{aligned}
$$

with $\gamma_{1} \leq 1.2, L_{B}=1.1 \times 10^{46} \mathrm{erg} / \mathrm{s}$. The cosmological parameters ${ }^{6}$ used by Chiang \& Mukherjee are $\Omega_{M}=1, \Omega_{\Lambda}=0$, and $H_{0}=75 \mathrm{~km} / \mathrm{s} / \mathrm{Mpc}$.

\subsubsection{Blazar spectra}

From observations it is known that the spectra of EGRET blazars can be characterized in the energy range $100 \mathrm{MeV}<E<30 \mathrm{GeV}$ by single power laws with an average photon spectral index of $-2.15 \pm 0.04$ [196]. However, the measured index of some of the blazars differs significantly from the mean value (by more than three standard deviations in 2 out of 51 blazars; figs. 4 and 5 of [196]), which suggests that scatter

\footnotetext{
${ }^{6}$ As explained before, the impact of this (incorrect) cosmological parameter set in the number of detected blazars is small. The original values from the model are preserved here for simplicity.
} 
in the distribution of blazar spectra is real, and not just an artifact from statistical fluctuations. The simulation takes this into account.

To date, most of the EGRET blazars have not been detected by ground-based telescopes at (sub-TeV) energies. Although the intergalactic attenuation discussed in this dissertation could account for the lack of detection of distant sources $(z>0.5)$, there is a significant sample of low redshift EGRET blazars that are bright but remain undetected at $\mathrm{TeV}$ energies. This indicates a spectral break or rolloff taking place between the EGRET energy range and the TeV regime. Further evidence comes from the fact that most of the detected $\mathrm{TeV}$ blazars belong to the same subset of blazars, the X-ray selected BL Lac (XBL) objects (or High-peaked BL Lacs ; HBLs), that represent only a small fraction of the sources seen with EGRET. All of this suggests an intrinsic spectral rolloff for FSRQ-type blazars. Unfortunately, with little observational data in the $30-300 \mathrm{GeV}$ range, no firm conclusions can be drawn about the precise shape of the spectra. This is one of the motivations for the next generation of ground based experiments and GLAST.

Considering the likelihood of this spectral rolloff, blazars are simulated with two types of spectra: single and broken power laws. In the first case spectral indexes normally distributed around -2.15 with standard deviation of 0.04 are used, representing a situation without intrinsic rolloff in the GLAST energy range. To model intrinsic rolloffs, the analysis is repeated with a sample of blazars whose unredshifted spectra have a broken power law with mean index -2.15 below $50 \mathrm{GeV}$ and -3.15 above, again with a standard deviation of 0.04 in each case.

\subsubsection{Simulated population of blazars}

Combining the luminosity functions described above with the GLAST point source sensitivity, a reasonable prediction can be made above the number of blazars that 
will be observed with the instrument. It is important to keep in mind that these calculations are necessary only to show the performance of the technique, and that all this information will be replaced by the data GLAST itself provides.

The first step is to generate separate versions of the blazar sky with redshift and luminosity dictated by each luminosity function. For the Stecker \& Salamon luminosity function (SS_LF hereafter), the luminosity function is integrated in order to calculate the number of blazars with a flux above ${ }^{7} 1.5 \times 10^{-9}$ photons $\mathrm{cm}^{-2} \mathrm{~s}^{-1}$ for $E>100 \mathrm{MeV}$ (5 $\sigma$ point source flux sensitivity expected to be reached by GLAST within 2 years). This results in $\sim 12000$ likely sources, before any observational selections. For the Chiang \& Mukherjee luminosity function (CM_LF hereafter) a representative number of 10000 blazars was generated with redshifts in the range 0 $<\mathrm{z}<5$ according to the solid plot in fig. 5.3. The flux for each source was then calculated as

$$
F=L \frac{(1+z)^{2-\alpha}}{4 \pi d_{l}^{2}(z)}
$$

where $\alpha$ is the photon spectral index (gaussian distributed) and $d_{l}$ is the cosmological luminosity distance ${ }^{8} d_{l}(z)=\frac{2 c}{H_{0}}(1+z)\left[1-(1+z)^{-1 / 2}\right]$.

Galactic and extragalactic diffuse backgrounds were added to the observed fluxes of each blazar, assuming an exposure equivalent to two full years. The Galactic backgrounds were derived from the diffuse model used in EGRET analysis [121], while the extragalactic background component was modeled as an isotropic flux with an intensity of $4 \times 10^{-6}$ photons $\mathrm{cm}^{-2} \mathrm{~s}^{-1} \mathrm{sr}^{-1}$ for $\mathrm{E}>100 \mathrm{MeV}$ and a power law index of -2.15, under the assumption that a significant fraction of the EGRET isotropic

\footnotetext{
${ }^{7}$ The expected point source sensitivity for GLAST has been revised since publication of this analysis. The current estimate is $4 \times 10^{-9}$ photons $\mathrm{cm}^{-2} \mathrm{~s}^{-1}$ for $E>100 \mathrm{MeV}$ after 1-year-long observation in sky-survey mode. This value is obtained assuming a diffuse background flux of 1.5 x $10^{-5} \mathrm{~cm}^{-2} \mathrm{~s}^{-1} \mathrm{sr}^{-1}$ (i.e. as observed by EGRET). Nevertheless, GLAST is expected to resolve a significant fraction of the diffuse background into point sources, and this would lead to a better point source sensitivity. Thus, the point source sensitivity used in the analysis could still be achieved.

${ }^{8}$ For consistency, the luminosity distance is calculated with the same cosmological parameters used for the luminosity functions.
} 


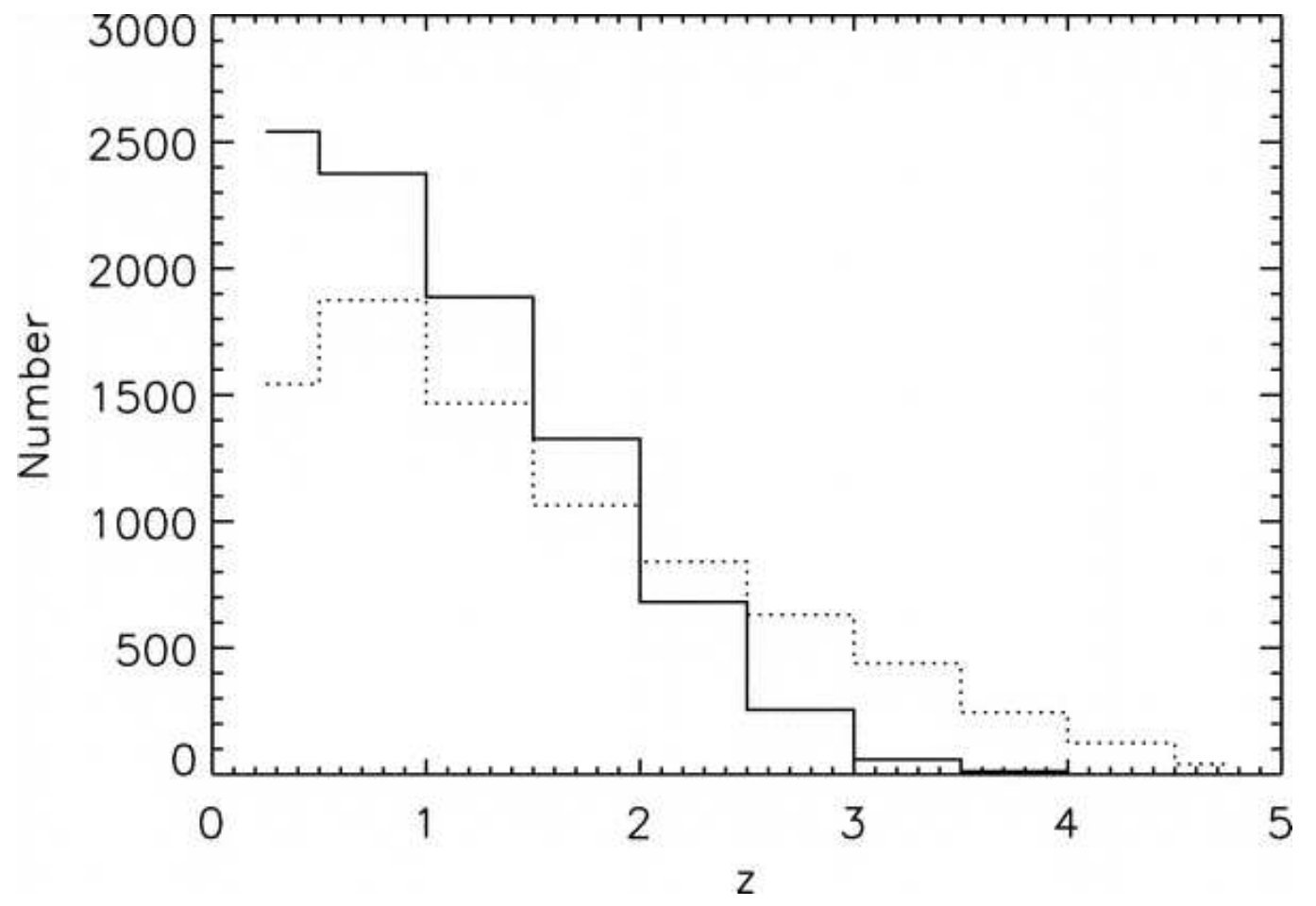

Figure 5.4: Number of detectable blazars with GLAST in each redshift interval. The conditions for blazar detectability are defined as: i) a $5 \sigma$ excess above the diffuse background flux at $E>1 \mathrm{GeV}$, and ii) location outside the galactic plane $\left(|b|>10^{\circ}\right)$. The solid curve is the population according to Stecker \& Salamon Luminosity Function, and the dotted line corresponds to the model by Chiang \& Mukherjee. 
background will be resolved by GLAST. Also included was the redshift dependent absorption above $10 \mathrm{GeV}$. The form of this dependence was parametrized from the EBL models by Stecker \& Salamon (1998) and by Primack et al (1999) discussed in Chapter 2 (EBL models are reviewed in section 2.5).

Any blazar within $10^{\circ}$ of the galactic plane or whose observed flux was less than $5 \sigma$ above the background flux at $\mathrm{E}>1 \mathrm{GeV}$ was removed from the sample. After these observational selections $\sim 9100$ blazars are left when using SS_LF, and $\sim 8200$ when using CM_LF. Figure 5.4 shows a histogram of the number of blazars in each 0.5 redshift bin. CM_LF predicts a population of blazars that are intrinsically brighter when compared to the population from SS_LF. In that case, GLAST would detect more blazars at higher redshift, as can be observed from the graph.

\subsubsection{Calculating the Flux ratios}

To detect the attenuation of blazar gamma ray emission by EBL absorption, the ratio between observed fluxes for $\mathrm{E}>10 \mathrm{GeV}$ and $\mathrm{E}>1 \mathrm{GeV}$ is calculated for each blazar,

$$
\frac{F(E>10 G e V)}{F(E>1 G e V)}
$$

where these observed fluxes are calculated using Poisson distributions equivalent to two full years of exposure. This quantity has the following characteristics:

- Simple to calculate.

- Very sensitive to EBL attenuation for most EBL models. As explained in Chapter $2, \gtrsim 10 \mathrm{GeV}$ photons have the possibility to experience (given the redshift at which they are produced) a broad range of optical depths, from strong attenuation $(\tau \gtrsim 5)$, to no absorption whatsoever $(\tau \sim 0)$. In contrast, photons with energy below $10 \mathrm{GeV}$ suffer no attenuation, independently of the source redshift. 
- Independent of blazar brightness.

- If no EBL absorption is present, the flux ratio depends only on the spectral index.

- Still useful with rolloffs above $50 \mathrm{GeV}$ at the source. Since the contribution of photons above $50 \mathrm{GeV}$ to the integral flux is small.

A mean spectral index of -2.15 yields a flux ratio $F(E>10 \mathrm{GeV}) / F(E>1 \mathrm{GeV})$ of $\sim 0.07$; therefore, when calculating the flux-ratio as a function of redshift, any deviation from this value will hint the effect of EBL attenuation. In the same way, different EBL models will result in different ratios as a function of redshift, making of this technique a powerful tool to distinguish and constraint present and future EBL models.

The error in each flux ratio is given by

$$
\sigma_{\text {ratio }}=\frac{1}{F(E>1 G e V)} \times \sqrt{\sigma_{F(E>10 G e V)}^{2}+\left[\frac{F(E>10 G e V)}{F(E>1 G e V)} \sigma_{F(E>1 G e V)}\right]^{2}}
$$

where $\sigma_{F}$ is the statistical error of the flux measurement in each energy range. Figure 5.5 presents a scatter plot of the flux ratio of each blazar with its corresponding error as a function of redshift.

\subsubsection{Results}

The crosses and triangles in Fig. 5.6 show respectively the weighted mean ratio in each redshift bin when using the EBL models by Salomon \& Stecker (1998) and Primack et al (1999). To avoid the bias of small number Poisson statistics toward lower values, the flux ratio of each source was weighted by the Poisson error of the $\mathrm{E}>1 \mathrm{GeV}$ flux, rather than the formal, propagated error of the flux ratio. For comparison, the diamonds show the same ratio when the intergalactic absorption is 


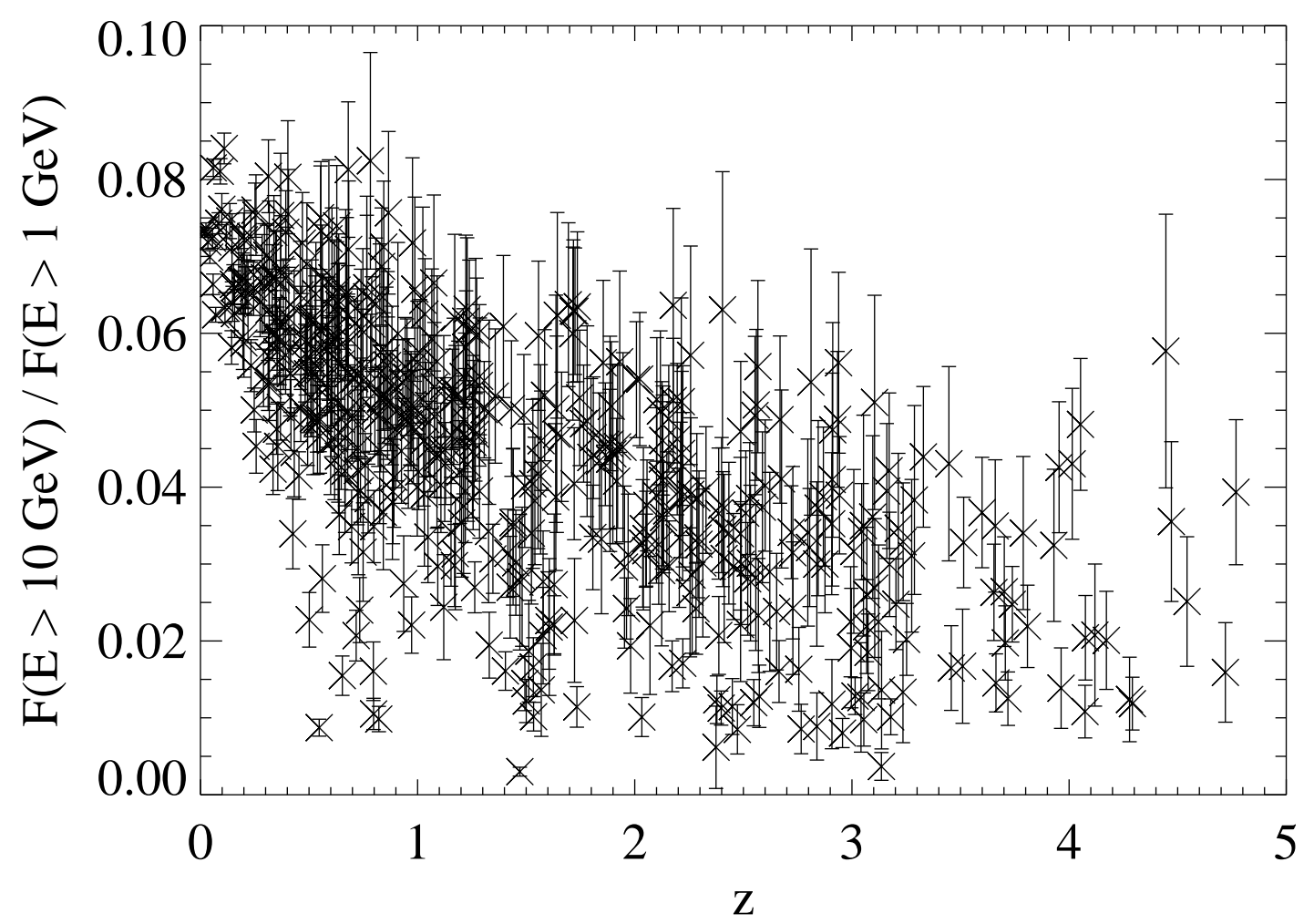

Figure 5.5: Flux ratio as a function of redshift using the Chiang and Mukherjee luminosity function and the EBL model by Salamon \& Stecker (1998). Each data point corresponds to the flux ratio of a blazar with an error bar given by eq. 5.8. For better clarity, only one out of every 20 blazars in the simulation is shown in this figure. 

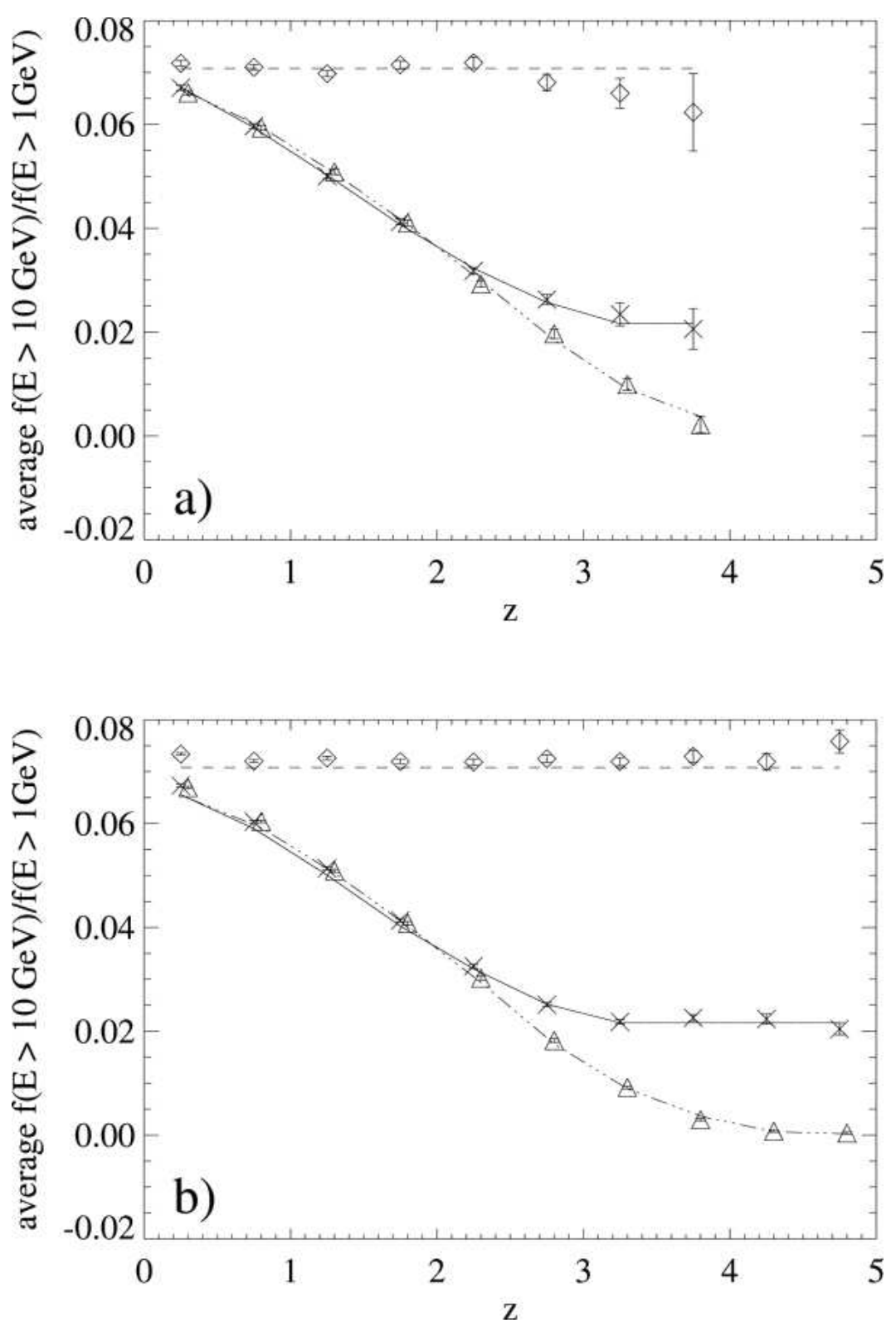

Figure 5.6: Mean observed flux ratio using the luminosity function by (a) Stecker \& Salamon [255] and (b) Chiang \& Mukherjee [43]. Each cross is the mean observed flux ratio in the corresponding redshift interval with fluxes attenuated by the EBL model by Salamon \& Stecker (1998) [233]. The solid curve is the ratio calculated analytically with the same model. The triangles and the dash-dotted line are the mean ratio and analytically calculated flux ratio for blazars, respectively, with the EBL model by Primack et al (1999) [223]. The diamonds on the top correspond to the mean observed flux ratio with no EBL attenuation, while the dashed line is the corresponding analytical ratio. 
removed from the observed blazar fluxes. The analytically derived flux ratio using each model are plotted as a solid line (Kneiske's best fit) and as a dot-dashed line (Kneiske's High-UV); the dashed lines correspond to the case with no attenuation. In all cases, the error bars are statistical, obtained by computing the RMS scatter within each redshift bin and dividing by $\sqrt{N}$.

The results shown here demonstrate the power of the technique. It is evident from the figure that the flux-ratio values obtained with each model are different, but most important that the decrease in flux ratio due to EBL is observable starting at $z=1$ (with respect to the case with no EBL attenuation). This indicates, assuming the availability of gamma ray sources and sufficient EBL density, that blazars can be used to effectively probe the EBL density at cosmological redshifts. Oh [210] performed an independent calculation of the opacity of gamma ray blazar emission to pair production by UV photons as a function of redshift. Although he does not link his calculations to GLAST capabilities, he obtains attenuation factors that vary strongly with redshift in a manner roughly consistent with the calculations used here.

As mentioned before, the analysis is repeated with the blazar spectra changed from single power law with mean index -2.15 , to broken power laws with mean index -2.15 below $50 \mathrm{GeV}$ (at the source) and -3.15 above. The results are plotted in Figure 5.7 following the same conventions used in fig. 5.6. Since the break energy is defined at the source, the ratio obtained without EBL absorption (diamonds and dashed line) is not constant as a function of redshift.

Fewer blazars have a detected flux above $10 \mathrm{GeV}$; however, the effects of absorption are still evident. Sources with no detectable flux above $10 \mathrm{GeV}$ (zero photons) still provide valuable information; indeed, neglecting them introduces a bias. The modified $\chi^{2}$ statistic used here (from [189]) accounts for those sources.

Since publication of the flux-ratio method [42], new and updated EBL models have become available in the literature. The analysis has been repeated with these new 

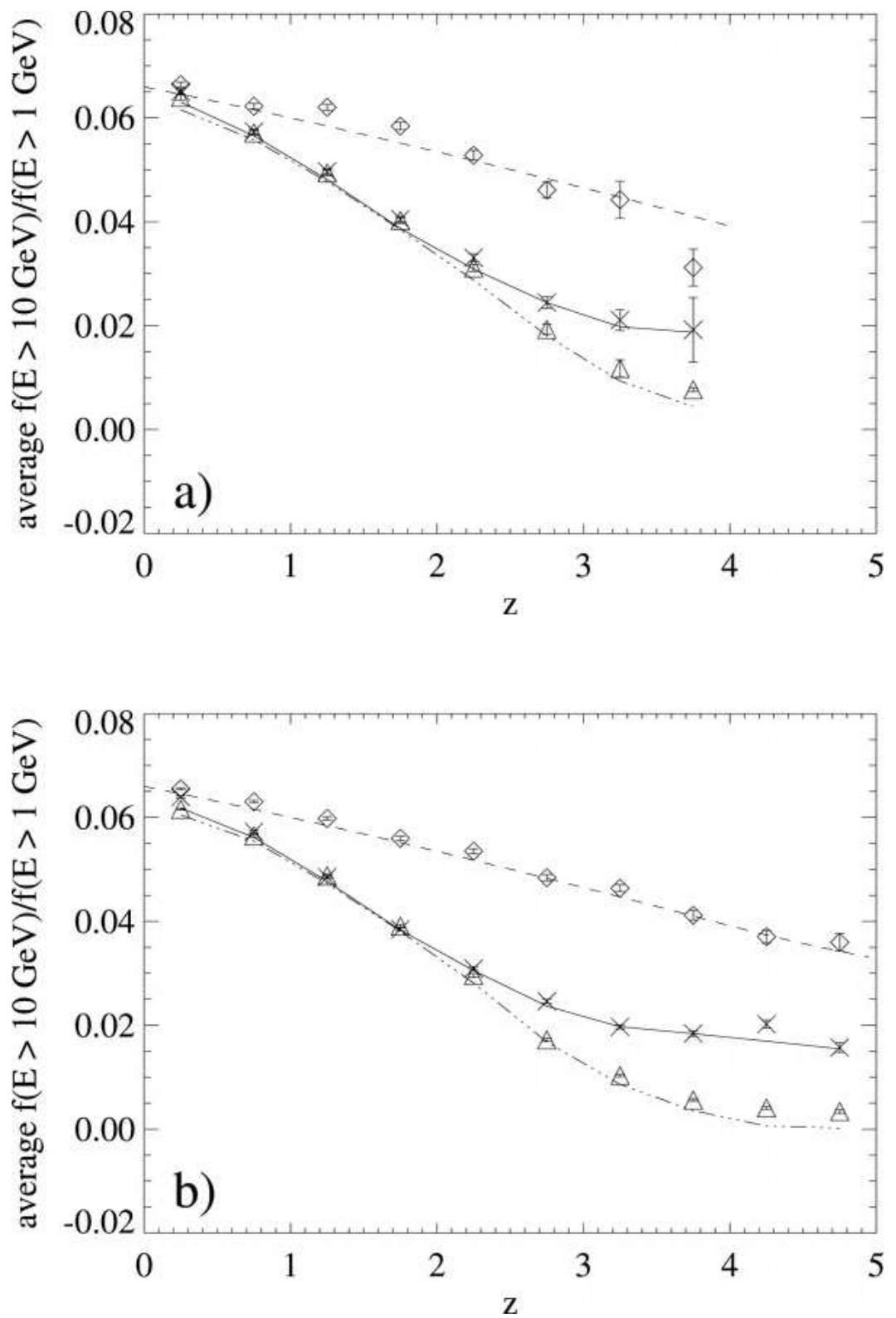

Figure 5.7: Mean observed flux ratio for blazars with a broken power-law spectrum (break energy set to $50 \mathrm{GeV}$ at the source). The luminosity function is obtained from (a) Stecker \& Salamon [255] and (b) Chiang \& Mukherjee [43]. The EBL attenuation is given by either Salamon \& Stecker (crosses) or Primack et al EBL models (triangles). The analytically calculated flux ratios for each luminosity function are shown by the solid and dot-dashed lines respectively. The dashed line and the diamonds show the same results in the case of no EBL attenuation. 
EBL models (keeping the same blazar populations) and the results are summarized in fig. 5.8. It can be deduced from the plot that despite the observational and theoretical advances in the last decade, the opacity predictions by competing EBL models diverge even more nowadays. This highlights the importance of GLAST observations for the study of the EBL at high redshifts.

From figures 5.6 and 5.7 it is evident that detecting the attenuation of blazar gamma ray emission by the EBL is feasible; with the condition that a large sample of gamma ray sources is available, especially at high redshifts. The analysis tool explained here, applied to the large population that GLAST will observe can be used to differentiate intrinsic single blazar peculiarities from the redshift dependent EBL absorption. Indeed, the obtained results indicate that this redshift dependence should be easily detectable, even when the diffuse background is taken into account and possible high-energy intrinsic rolloffs are considered.

\subsection{Spectral Analysis}

An alternate method to the flux-ratio method is presented in this section. This technique consists of measuring the spectrum steepening of individual blazars by means of a functional form with adjustable parameters, which is fit to the observed spectrum by the method of maximum likelihood. By studying the collective steepening (via fit parameters) of the sources as a function of redshift, it is possible to measure or at least constrain the effects of intergalactic absorption by the EBL.

Spectral source modeling has been used before to determine the strength of EBL attenuation of blazars $[68,150,271]$. The analysis presented here is different, however, because in order to determine the effects of EBL attenuation, it relies on the collective behavior as a function of redshift of the large number of blazars that GLAST is expected to detect. 

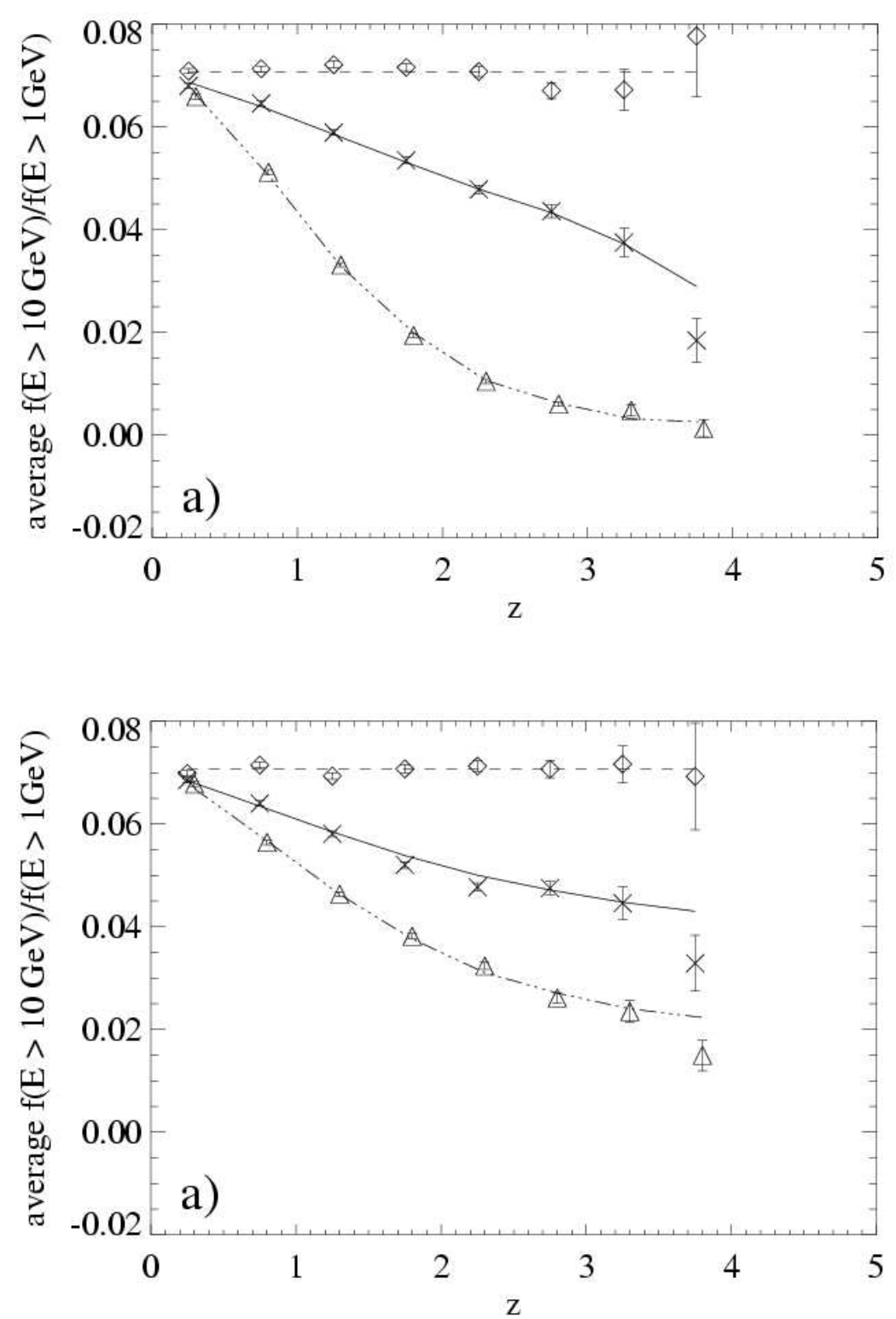

Figure 5.8: Mean observed flux ratio using the luminosity function by Stecker \& Salamon [255]. In the top plot, the EBL attenuation is given by either Primack et al (2005; crosses) (sec. 2.5.2.2, [222]) or Stecker et al (2006 ; triangles) (sec. 2.5.2.3, [253]). In the bottom plot, the EBL attenuation is given by Kneiske - best fit (crosses) and and Kneiske - High UV (triangles) (sec. 2.5.2.1, [144]). The analytically calculated flux ratios for each luminosity function are shown by the solid and dotdashed lines respectively. The dashed line and the diamonds show the same results in the case of no EBL attenuation. 


\subsubsection{Monte Carlo Simulation}

The analysis introduced in this section relies on the capabilities of the LAT to obtain well measured spectra for a relatively large number of bright $\gamma$-ray sources. The simulation used to demonstrate this analysis should therefore be more detailed and realistic in what regards to blazar emission. A 1-year-long simulation of the $\sim 300$ brightest blazars expected to be detected with GLAST was produced. The simulation also included galactic and extragalactic diffuse background, and a detailed model for the variability of blazars. Absorption by the EBL was simulated using the best-fit model by Kneiske et al (sec. 2.5.2.1; [144]).

\subsubsection{Blazar Population}

\section{EGRET Blazars}

The brightest objects in the simulation are the blazars from the 3rd EGRET cata$\log$ [106]. From the catalog, 105 sources are identified as blazars by the Candidate Gamma-Ray Blazar Survey (CGRaBS) [247] (discussed in section 5.4.2). The 105 sources consist of 102 FSRQs and 3 BL Lacs.

\section{Population Synthesis Model}

The population synthesis code by Giommi et al. [93] provided other bright blazars for the simulation. The code considers separately the radio luminosity functions for LBLs, HBLs and FSRQs, and also assumes pure luminosity evolution for FSRQs (see section 5.1.1)

$$
L(z)=L(0) e^{2.2 z /(1+z)}
$$

while no evolution is assumed for BL Lacs. With this as an input, the redshift and radio luminosity for the blazar population is obtained by Monte Carlo simulation. Next, the radio flux is used to extrapolate the gamma-ray flux, while taking into consider- 
ation the duty cycle of blazars, which is constrained by the requirement that blazars as a population should not overproduce the extragalactic radiation background, not only at high energies, but in the microwave band as well [94].

\subsubsection{Blazar Variability}

Among the AGN sub-classes, blazars are distinguished for their strong variability across the electromagnetic spectrum. This variability manifests itself in the form of flares with varying amplitudes on a wide range of timescales that can be caused by internal shocks in the jet [248], ejection of relativistic plasma into the jet [174], or magnetic reconnection events in a magnetically dominated jet [160], among others.

For the simulation, the blazar light curves are generated with the code by Tosti et al. [267]. This code implements a phenomenological method developed by Timmer \& Koenig [264] to reproduce the stochastic behavior observed in the lightcurves of blazars, which can be well-characterized by a power-law power spectrum density (PSD):

$$
P S D(f) \propto f^{-\alpha}
$$

where $f$ is the frequency, and where the power-law index $\alpha$ has a value $\sim 2-3$ in the case of blazars [139]. One of the lightcurves obtained with this method is shown in fig. 5.9 as an example.

In summary, the simulation contains:

- 105 identified blazars from the 3rd EGRET catalog (CGRaBS [247])

- Bright sources (flux greater than $1.5 \times 10^{-7} \mathrm{~cm}^{-2} \mathrm{~s}^{-1}$ ) from the Giommi et al. code.

- 150 FSRQs were assigned broken-power-law spectra (energy break at $\sim 50$ $\mathrm{GeV})$ 


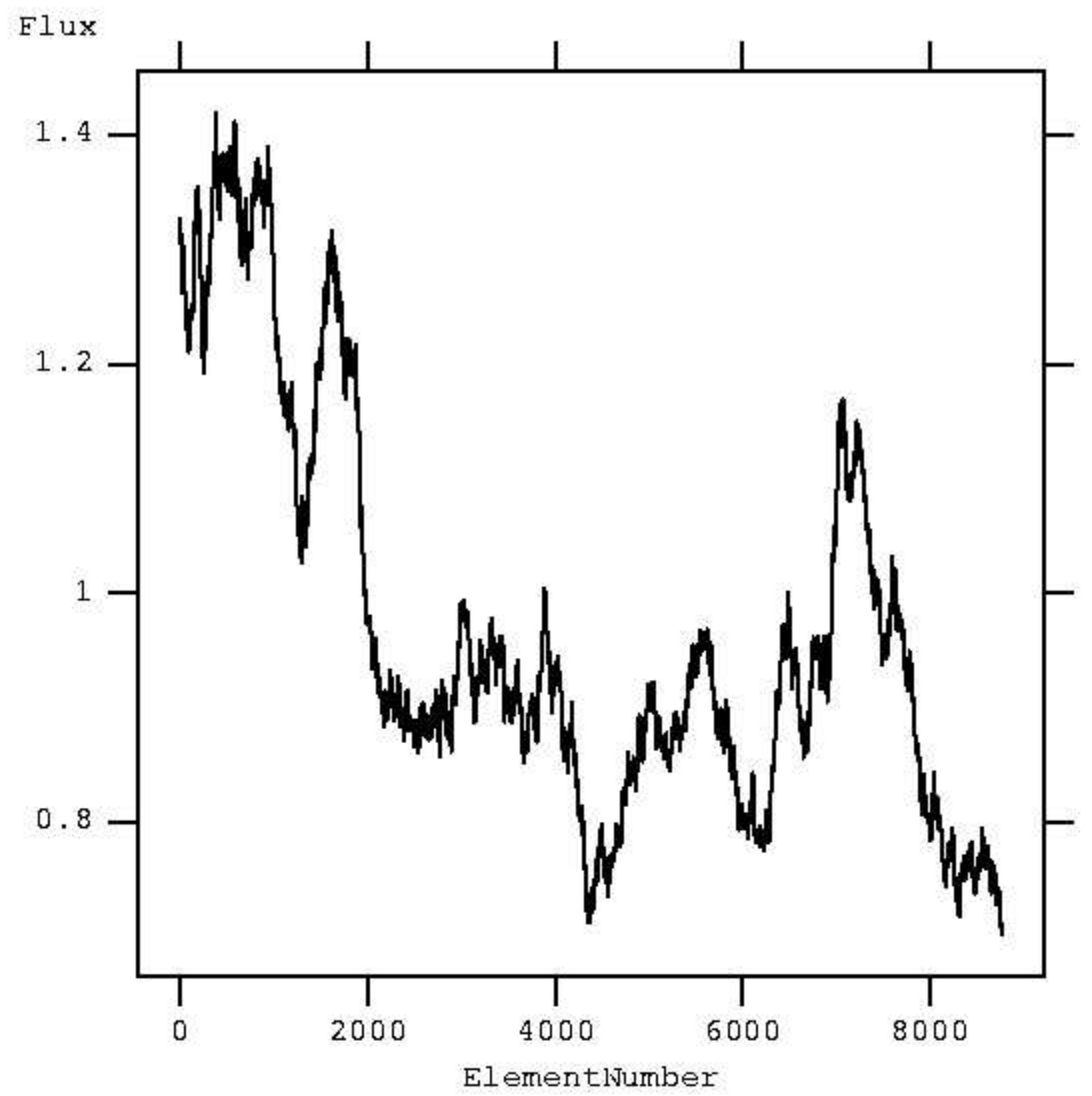

Figure 5.9: Simulated blazar lightcurve, from [267]. The time interval of the simulation is one year, although the $\mathrm{x}$-axis is expressed in arbitrary units. 
- 50 BL Lacs were assigned power-law spectra

- Galactic diffuse emission according to the GALPROP model by Moskalenko et al [193]

- Extragalactic diffuse background with intensity $1.55 \times 10^{-5} \mathrm{~cm}^{-2} \mathrm{~s}^{-1} \mathrm{sr}^{-1}(\mathrm{E}>$ $100 \mathrm{MeV}$ ), which is consistent with the EGRET measurement [46].

\subsubsection{Modeling the observed blazar spectrum}

The flux attenuation of extragalactic sources is expressed in terms of the optical depth introduced in Chapter 2:

$$
\left(\frac{d N}{d E}\right)_{\text {observed }}=\left(\frac{d N}{d E}\right)_{\text {intrinsic }} \times e^{-\tau}
$$

where $\tau$ is a function of the photon's observed energy $E$ and the redshift $z$ of the source $\tau=\tau(E, z)$. If a functional form is to be used to approximate the attenuated spectrum of a source at a given redshift, this functional form should be able to reproduce the different possible realizations of $\tau(E, z)$ while restricting the number of free parameters to a minimum.

The attenuated flux of blazars observed at $\mathcal{O}(\gtrsim 100 \mathrm{GeV})$ energies has been characterized before in the literature $[68,150]$ as

$$
\left(\frac{d N}{d E}\right)_{\text {observed }}=\Gamma E^{-\alpha} \times e^{-E / E_{0}}
$$

where $\alpha, \Gamma$, and $E_{0}$ are free parameters whose value is determined by fitting the model to the data. It should be noted, that the term $\Gamma E^{-\alpha}$ (where $\alpha$ is the spectral index and $\Gamma$ is the overall normalization), is the single power law function that is commonly used to describe the spectrum of blazars to first approximation (more on 


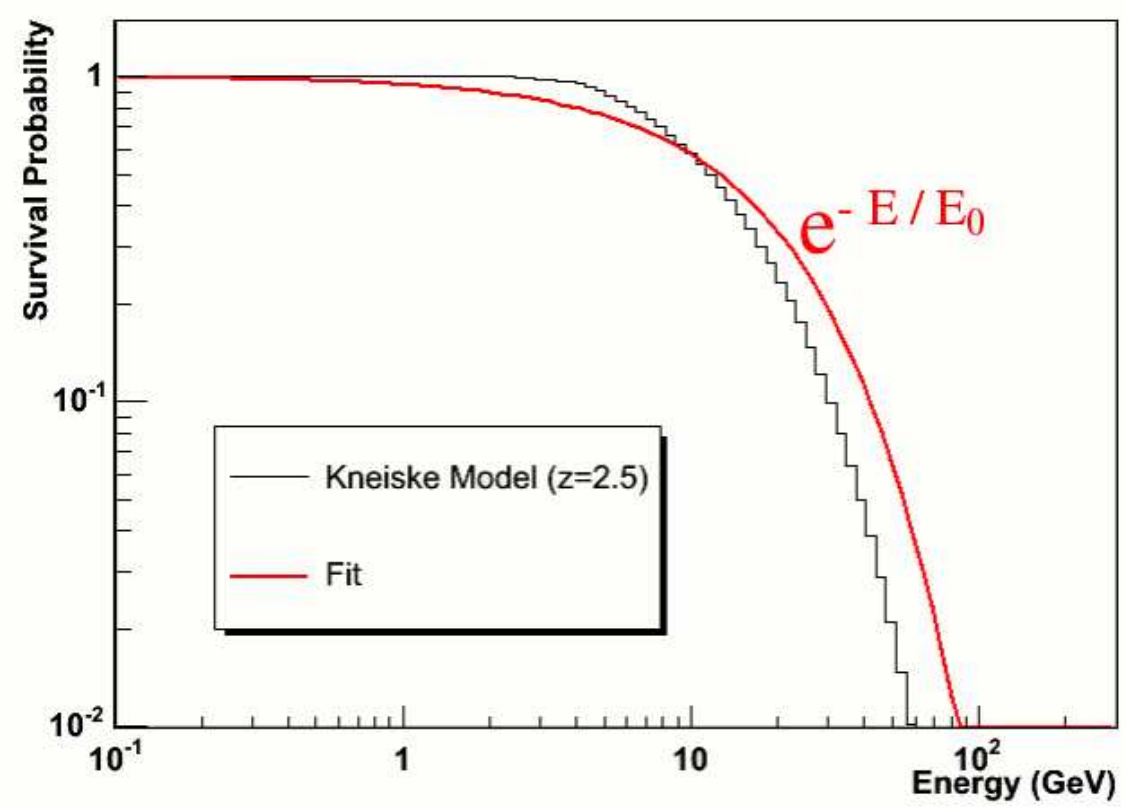

Figure 5.10: Survival probability $\left(e^{-\tau}\right)$ for a $\gamma$-ray emitted at redshift $z=2.5$ according to the best-fit EBL model by Kneiske et al (black line). The fit obtained with a function of the form $e^{-E / E_{0}}$ is also shown in red. This function does not fit the data well because it cannot reproduce the fact that $\tau$ is effectively zero at low energies $(E<10 \mathrm{GeV})$ and then increases rapidly at some particular energy that depends on the redshift of the source. Thus, a different function (eq. 5.12) is used to model the optical depth.

this below). If one takes this parametrization choice at face value, the $\exp \left(-E / E_{0}\right)$ term is expected to reproduce the spectrum steepening due to EBL absorption.

The adequacy of this parametrization model was tested for the optical depth values expected in the LAT energy range at intermediate and high redshifts. The model does not fit the data well as can be seen in fig. 5.10. The reason for this is that $e^{-\left(E / E_{0}\right)}$ cannot reproduce the behavior of $\tau$, which is effectively zero in the low end of the LAT energy range $(\mathrm{E}<\sim 1 \mathrm{GeV})$, and then increases rapidly $\Delta \tau / \Delta \log E \gg 1$ at some particular energy above $10 \mathrm{GeV}$ which depends on the redshift of the source (see fig. 2.7 in Chapter 2).

A much better result can be obtained by introducing an additional degree of freedom to the original function in the following way 


$$
\begin{array}{cc}
e^{-\left(E-E_{b}\right) / P_{1}} & \text { if } E>E_{b} \\
1 & \text { if } E<E_{b}
\end{array}
$$

This parametrization has proven useful under different EBL models and different redshifts for the level of attenuation that $\gamma$-ray sources observed by the LAT are expected to experience. The survival probability $e^{-\tau}$ as a function of the energy and its respective fit with the proposed function can be seen in fig. 5.11. Therefore, the functional form proposed here to represent the observed spectrum of EBL attenuated blazars is given by:

$$
\begin{array}{ccc}
\Gamma E^{-\alpha} & \text { if } & E<E_{b} \\
\Gamma E^{-\alpha} \times e^{-\left(E-E_{b}\right) / P_{1}} & \text { if } & E>E_{b}
\end{array}
$$

There are two features in the expression above that will affect the analysis. The first one is the choice of $\left(E-E_{b}\right) / P_{1}$ as a good approximation ${ }^{9}$ to $\tau$. It is important to realize that the optical depth $\tau$ is a function of the EBL density $n_{E B L}$, and therefore summarizes the energy output over cosmic time of all the galaxies in the visible universe, with each galaxy having a peculiar star formation history (with some galaxies having accretion rate history as well) and thus, a spectral energy distribution. The EBL models considered in Chapter 2 attempt to address all these issues. The complexity of the physical processes that give origin to the EBL also make $\tau(E, z)$ a complex quantity. Although a parametrization of $\tau$ in terms of physical EBL parameters (such as mean number of photons, mean energy, etc.) would be highly desirable,

\footnotetext{
${ }^{9}$ In the case of a very bright source with a very large number of detected photons one could envision a scenario where more degrees of freedom are used to characterize the spectrum steepening more accurately.
} 


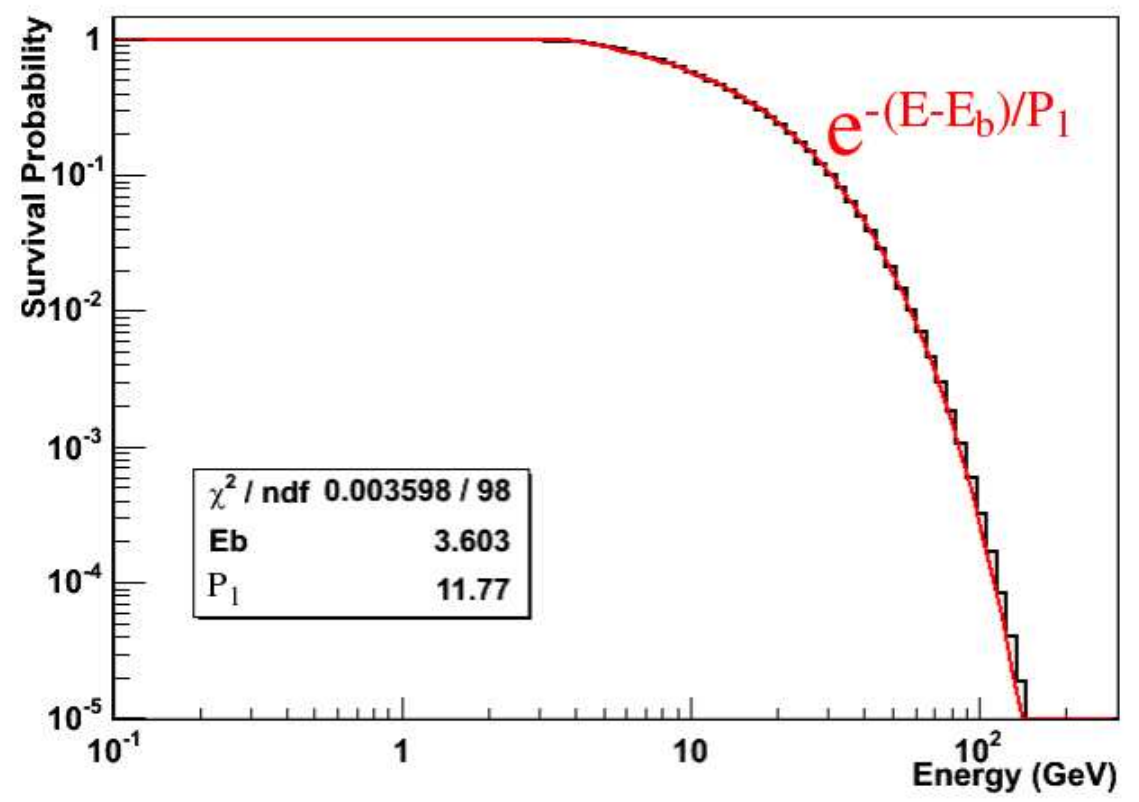

Figure 5.11: Survival probability $\left(e^{-\tau}\right)$ for a $\gamma$-ray emitted at redshift $z=2.5$ according to the best-fit EBL model by Kneiske et al (black line). This time a fit to the model is tried with the function $e^{-\left(E-E_{b}\right) / P_{1}}$ (red line). The insert contains the $\chi^{2} /$ ndf and the values of $E_{b}$ and $P_{1}$ obtained from the fit.

this has not been developed yet and might not be feasible given the intrinsic complexity of $n_{E B L}$. A parametrization like the one presented above is just an approximation, and therefore, is intended only to describe within observational uncertainties the observed spectrum of a source after EBL absorption. For each blazar, the values of $E_{b}$ and $P_{1}$ obtained from the fit will be used to calculate the energy cutoff due (in principle) to EBL attenuation. The correlation with redshift of the energy cutoffs obtained this way can be used to distinguish between different EBL models.

The second choice in the expression above that needs to be discussed is the explicit assumption that the unattenuated spectrum of blazars can be well approximated by a single power law. At first sight, this might seem a bad choice since it is known that blazar spectra are considerably more complex, exhibiting curvature and variable intensity [6]. Furthermore, strong radiation fields within the sources could lead to gamma-ray cut-off energies well below the EBL-induced ones [64]. The choice is 
justified, however, because such simple parametrization will allow the determination of the spectral steepening of a larger number of GLAST sources. Consequently, the bias introduced by individual sources would become less significant when considered together as a population.

An alternate analysis to the one presented here would require the calculation of the blazar intrinsic spectrum at $\gamma$-ray energies with state-of-the-art emission models that use observations at other wavelengths as an input. The difference between this predicted intrinsic spectrum and the contemporaneously ${ }^{10}$ measured $\gamma$-ray spectrum could then be used to estimate the effects of EBL absorption. For example, Coppi \& Aharonian [49] propose using X-ray observations in conjunction with Synchrotron Self-Compton (SSC) emission models to calculate the intrinsic spectrum of HBL objects observed by ground-based instruments at $\mathcal{O}(\gtrsim 100 \mathrm{GeV})$ energies. The problem with this type of approach, however, is that the current theoretical models are still far from reproducing the complexity of the blazar-emission mechanism(s) realized in nature. The richness of the AGN-phenomena is vast, with many issues still not understood, and often not even attempted to model [227]. Nonetheless, great progress will be made in the understanding of blazars and their emission processes with the advent of GLAST and the new generation of ground-based telescopes. This approach would constitute an independent type of analysis with respect to the one proposed here, and when considered together, they will validate and complement each other.

\footnotetext{
${ }^{10}$ Simultaneity in the observations would be desirable, but the long integration time required by the LAT to measure the high energy end of the source spectrum makes this impractical.
} 


\subsubsection{Spectrum fitting}

\subsubsection{Maximum Likelihood}

Analysis of LAT data is complicated by the relatively low photon detection rate and the highly variable LAT point-spread-function (PSF). The predominant method for analysis of LAT point sources (as it was for EGRET [182]) is the maximum likelihood technique (hereafter called likelihood). Likelihood is a well-known statistical technique used to quantify the relative extend to which the data support a statistical hypothesis [21]. When the hypothesis takes the form of a model with adjustable parameters, the likelihood can be expressed in terms of those parameters, and by maximizing the likelihood, one can find the parameter values that fit best the data. Hence, the expression "method of maximum likelihood".

For LAT point sources like blazars, the hypothesis usually consists of a point source spectrum $S_{P}(E)$, plus galactic and extragalactic diffuse backgrounds $S_{B}(E)=$ $S_{G a l}(E)+S_{E x}(E)$. The data density (i.e. photons) resulting from such spectrum (or any other by that matter) depends on the response of the instrument $R\left(\vec{x} ; \vec{x}^{\prime}\right)$ :

$$
M\left(\vec{x}^{\prime}\right)=\int d E d \vec{x} R\left(\vec{x} ; \vec{x}^{\prime}\right)\left[S_{P}(E)+S_{B}(E)\right]
$$

where $\vec{x}$ stands for the true properties of the signal according to the model (energy, direction, etc.) and $\vec{x}$ ' for the reconstructed ones. Consequently, the total number of photon detections predicted by the model is given by

$$
N_{\text {pred }}=\int d \vec{x}^{\prime} M\left(\vec{x}^{\prime}\right)
$$

and the Extended Maximum log-Likelihood (EML) can now be defined as [182]:

$$
\log (L)=\sum_{i} \log M\left(\vec{x}_{i}\right)-N_{\text {pred }}
$$


where the sum is over all the data. For the analysis of LAT data, likelihood has at least two very important applications. The first one is to determine the statistical significance of source detections through the "Test Statistic" (TS) test. The TS value is defined as the log likelihood ratio of two models $L_{1}$ and $L_{2}$

$$
T S \equiv-2 \log \left(\frac{L_{2}}{L_{1}}\right)
$$

where the $L_{2}$ hypothesis is that a point source exists at the position under consideration, and $L_{1}$ is the null hypothesis. The statistical significance of the candidate source can then be determined by treating TS as a $\chi^{2}$ value with one degree of freedom (Wilks's theorem [276]), or equivalently, treating $\sqrt{T S}$ as $n \sigma$, namely, the integral of the standard normal distribution from $n$ to $\infty$.

The second important application of the maximum likelihood method for analysis of LAT data, as already mentioned above, is to find the parameters of a given flux model that fit best the data. This application will be used below to look for signatures of EBL attenuation in the spectra of blazars.

The likelihood tool of the GLAST software gtlikelihood was used to fit the spectrum of the simulated sources. Figure 5.12 shows the spectral fit of one of the simulated blazars as an example. A power-law times $e^{-\left(E-E_{b}\right) / P_{1}}$ (functional form introduced above) provides a very good fit to the data. This example in particular, has a clear exponential cutoff in the spectrum that is due to the EBL attenuation routine introduced in the simulation.

If this spectrum had been obtained from actual data instead of a Monte Carlo simulation, the possibility would remain that the observed cut-off was a feature of the intrinsic spectrum of the source, resulting for example, from the presence of strong radiation fields within the source. The uncertainty about the true shape of the gamma-ray spectra -before EBL absorption has occurred- will always be the main 


\section{FSRQ \#8}

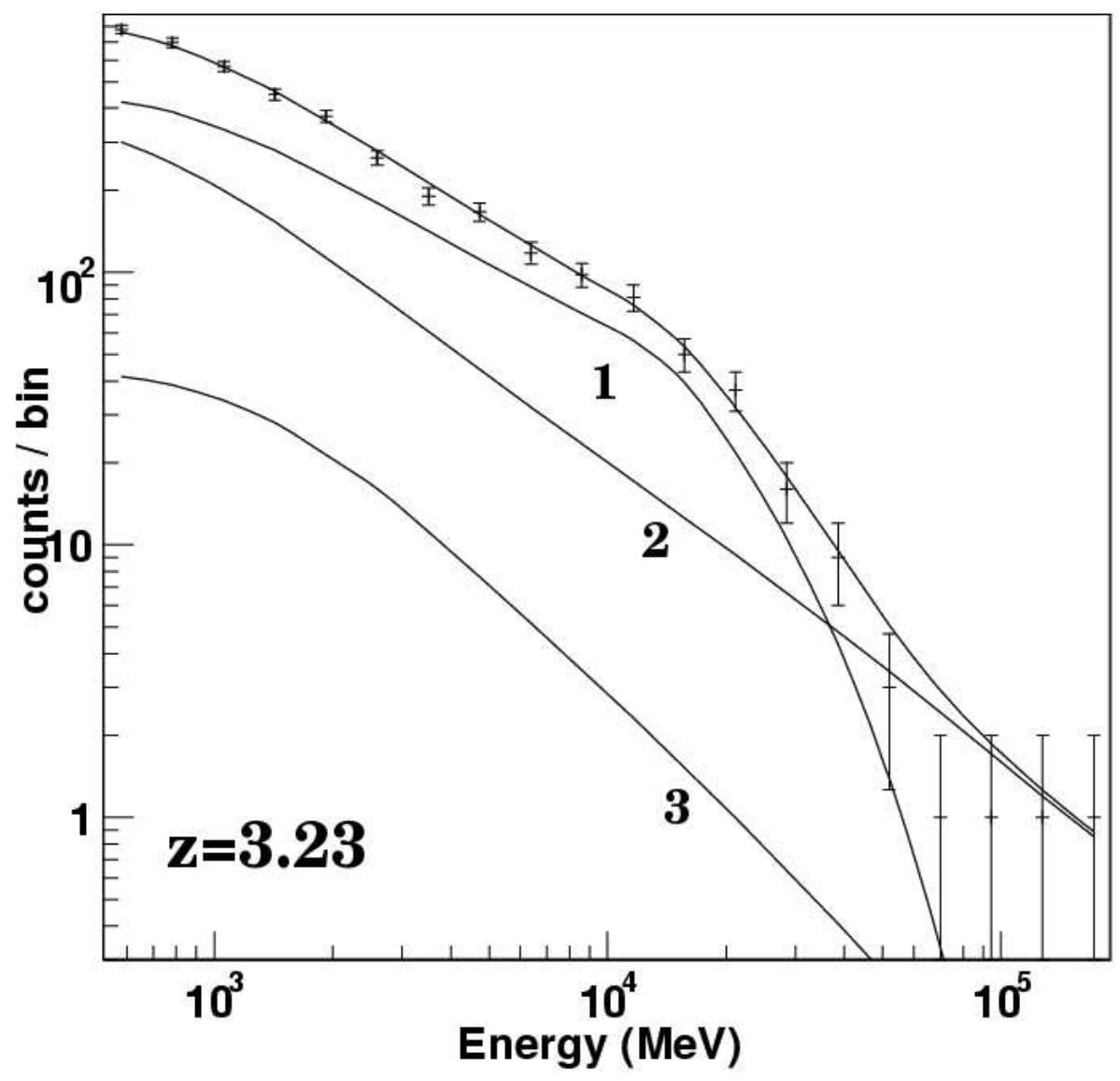

Figure 5.12: Spectrum and model fit for a simulated $\gamma$-ray blazar at redshift $z=3.23$. The solid lines correspond to: (1) point source with spectrum given by eq. 5.13, (2) extragalactic diffuse background, and (3) galactic diffuse background. Each spectrum is obtained from the convolution of the instrument response function with the source model. 


\begin{tabular}{|c|c|c|c|c|c|c|}
\hline Source & $z$ & $E_{b}(\mathrm{GeV})$ & $P_{1}(\mathrm{GeV})$ & $\sigma_{E_{b}, P_{1}}\left(\mathrm{GeV}^{2}\right)$ & $E_{0}(\mathrm{GeV})$ & $E_{M C}(\mathrm{GeV})$ \\
\hline \hline BL Lac \#26 & 0.89 & $33.3 \pm 6.5$ & $47.0 \pm 4.8$ & -7.52 & $80.3 \pm 7.1$ & 84.4 \\
\hline FSRQ \#104 & 1.47 & $23.4 \pm 5.3$ & $28.1 \pm 6.1$ & -1.64 & $51.5 \pm 7.9$ & 52.4 \\
\hline J1828+0142 & 1.77 & $15.4 \pm 1.2$ & $27.0 \pm 1.3$ & -0.3 & $42.4 \pm 1.6$ & 45.2 \\
\hline FSRQ \#48 & 3.91 & $12.4 \pm 5.4$ & $20.0 \pm 0.7$ & 0.5 & $32.5 \pm 5.4$ & 28.8 \\
\hline
\end{tabular}

Table 5.1: Fit parameters and spectral energy cut-offs for the sources shown in fig. 5.13

obstacle to any kind of analysis of EBL attenuation. Nevertheless, this obstacle may be overcome by collecting data from a large sample of sources (such as the future catalog of GLAST-detected blazars), because sources at similar redshifts would experience the same level of EBL attenuation.

\subsubsection{Fazio-Stecker Relation}

As already explained in Chapter 2, the relation $\tau\left(E_{\gamma}, z\right)=1$ where $\tau$ is the optical depth of the universe to $\gamma$-rays as a function of the observed $\gamma$-ray energy $E_{\gamma}$ and redshift z, was first introduced by Fazio \& Stecker [80] in 1970, and has been recently coined by [144] as the Fazio-Stecker relation (FSR).

Kneiske et al [144] have proposed to use the FSR to compare EBL models (such as those reviewed in Chapter 2) with the FSR distribution obtained from observations. The convergence (or lack thereof) of the theoretical expectations and the measured values can be used to validate or at least constrain EBL models. This idea is implemented here by considering the FSR obtained after determination of the e-fold cut-off energies of the brightest blazars expected to be observed with GLAST.

Taking the spectrum in fig. 5.12 as an example, the following parameter values and statistical errors are obtained when fitting to eq. 5.13,

$$
\begin{aligned}
& E_{b}=(13.66 \pm 1.97) \mathrm{GeV} \\
& P_{1}=(14.98 \pm 2.71) \mathrm{GeV}
\end{aligned}
$$



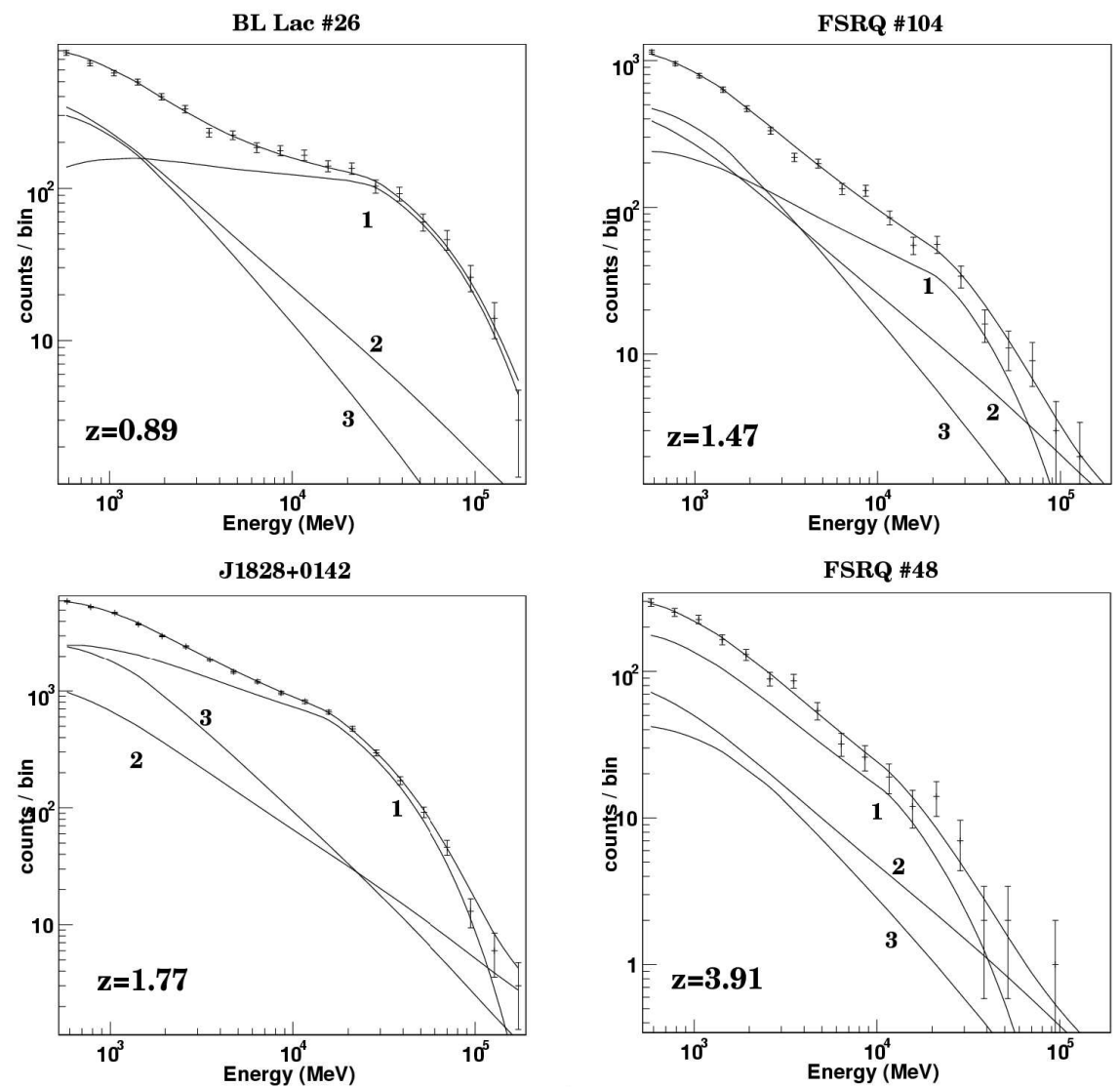

Figure 5.13: Spectra and model fits for blazars simulated at different redshifts with the same EBL model (Kneiske et al. best-fit). The functional form $\Gamma E^{-\alpha} \exp \left[\left(E-E_{b}\right) / P_{1}\right]$ is observed to fit well the blazar spectrum (1). Extragalactic (2) and galactic (3) diffuse background flux is also shown. Detailed inspection of the plots reveals the systematic spectrum steepening as a function of redshift. 
with a correlation factor in the error matrix of -1.48. The e-fold cut-off energy $E_{0}$ and its error $\sigma_{E_{0}}$ can now be determined from the fit parameters, which for this example yields

$$
\begin{aligned}
E_{0} & =E_{b}+P_{1} \\
& =28.64 \mathrm{GeV}
\end{aligned}
$$

and

$$
\begin{aligned}
\sigma_{E_{0}}^{2} & =\sigma_{E_{b}}^{2}+2 \sigma_{E_{b}} \sigma_{P_{1}}+\sigma_{P_{1}}^{2} \\
& =2.87 \mathrm{GeV}
\end{aligned}
$$

According to the Monte Carlo simulation, the true value of the energy cut-off is $E_{M C}=31.4 \mathrm{GeV}$ (Kneiske et al. best-fit EBL model). It is clear then, that at least for this example, the fit is able to quantify correctly the spectrum steepening due (in this case) to EBL attenuation. What follows now is to repeat the same analysis with all the blazars in the simulation. Figure 5.13 contains the spectra and model fits for a representative collection of such blazars. The corresponding energy cut-offs are presented in table 5.1.

Figure 5.14 presents the FSR scatter plot obtained from the analysis of the Monte Carlo simulation. The black squares indicate the energy cut-offs as determined from the fits (observations) and can be seen to reproduce very well the EBL model used for the simulation (Kneiske et al. - best fit). Not all the sources considered in the simulation produced meaningful fits: for some blazars the error in the determination of $E_{0}$ is greater than $E_{0}$ itself. This is due to the lack of photons at the highest energies for sources with soft intrinsic spectra $\left(\propto E^{-\alpha}\right.$; with $\alpha>2.5$. Of the 165 blazars included in the simulation with redshift $z>0.5$ (with EBL energy cut-offs 
that are in the energy range measured by GLAST) 97 of them yielded meaningful fits.

In the absence of intrinsic absorption within blazars and any type of spectrum curvature, the data points in the FSR plot will converge -amid statistical fluctuationsto the true curve $\tau(E, z)=1$ due to EBL absorption. If it turns out, however, that this is not the case for a few or most blazars, their measured cut-off energies would spread below the EBL-induced value, but never above. This would enable at least and upper limit on EBL attenuation (least-attenuated flux in a particular redshift range).

\section{$5.4 \quad$ Related Issues}

\subsubsection{Possible Sources of Bias}

\subsubsection{Selection Effects}

Analysis techniques like the two presented here will be affected by observational and selection effects, which will have to be addressed and quantified once the data is available. A list in order of apparent importance, from minor to major impact on the analysis, includes :

- Source misidentification. A $\gamma$-ray source can be misidentified (and therefore assigned a wrong redshift) when two or more source counterparts are present in its position error box. This is more likely to happen to dim sources, which will have larger error boxes. Misidentified blazars will appear as outliers in a FSR plot.

- Detection of distant (i.e. high-redshift) blazars is naturally biased towards highluminosity sources, which likely have high-accretion rates and could have strong radiation fields close to the acceleration site(s). This could lead to internal 


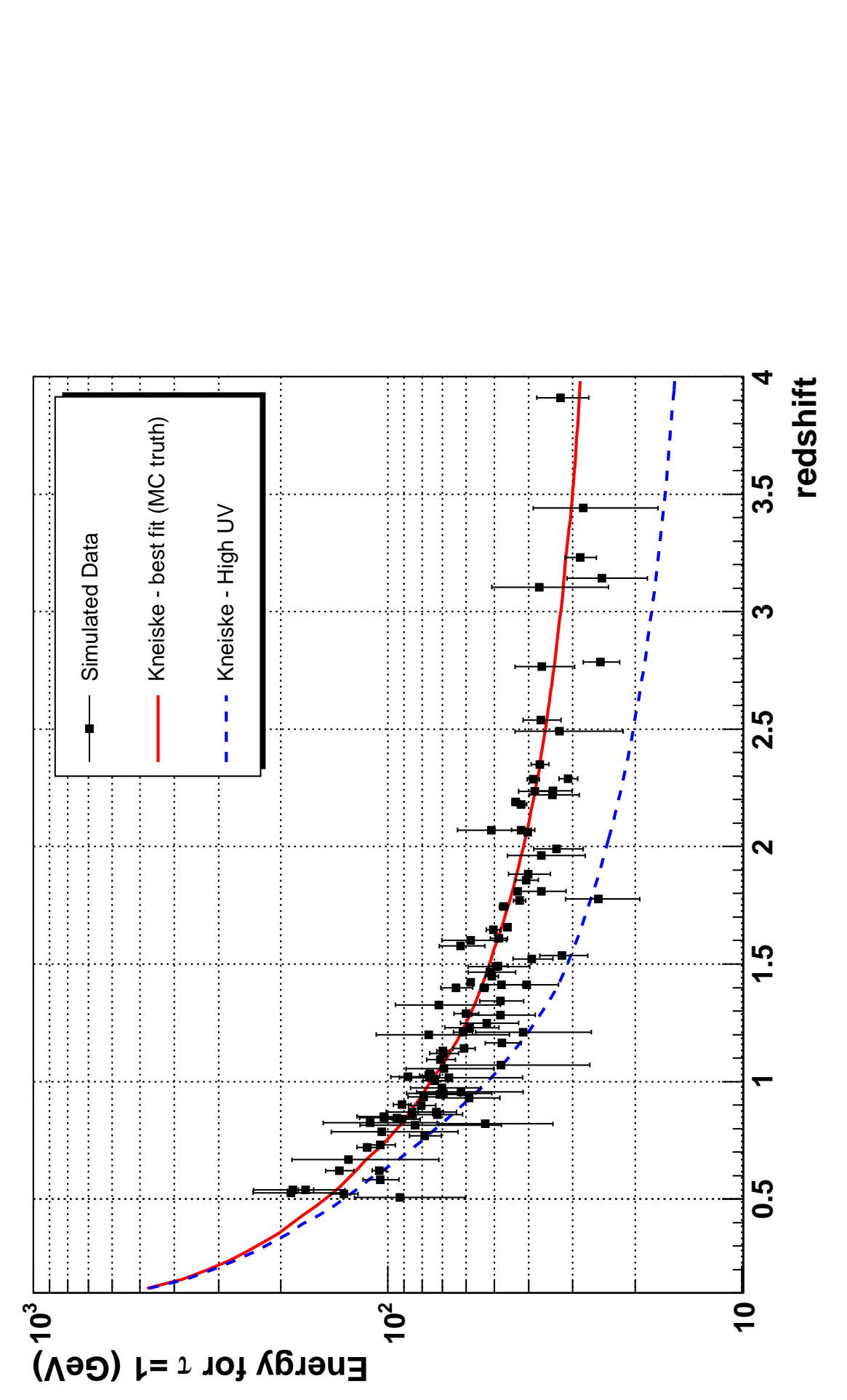

웅

घ.

.$\stackrel{\circ}{\Xi}$

记

园导

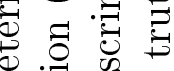

क

림

U

bo

㖓要

व.

政

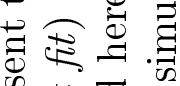

Wँ

눈

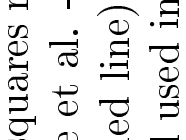

की

$\widetilde{\sigma}$

这焉

웡…王

형

完

荙国:

苛声范

क्षे

$g$.

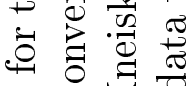

훙

䓔它志

政

ज结

武.

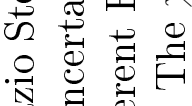

蛋

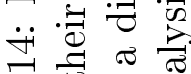

ம்

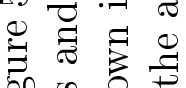
的等 $\frac{0}{\pi}$ 
absorption and cut-off energies that are systematically below the EBL-induced ones. The correlation of this selection effect with redshift could mimic the effect of EBL attenuation of blazars, leading to an overestimation of the EBL density at high redshifts (this scenario will be considered again in sec. 5.4.1.3).

- Related to the item above, the majority of blazars detected by GLAST are expected to be FSRQs, which are intrinsically brighter than BL Lacs. According to the current understanding of blazars, FSRQs are more likely to present strong radiation fields close to the acceleration site (narrow- and broad-line regions, etc.), leading to internal absorption of the $\gamma$-ray radiation [159]. If it turns out that most FSRQs are not suitable for EBL analyses due to this feature, the statistical precision of GLAST to probe the high-redshift EBL with blazars could be reduced since BL Lacs are not numerous at high redshifts (see redshift distributions in section 5.4.2), and its redshift determination is difficult because of the absence of optical lines in their spectrum.

\subsubsection{Blazar Variability}

Blazars display strong variability in their intensity and spectra. Indeed, observations of a few EGRET blazars indicated that the two may be correlated [197]. However, a recent study of all EGRET blazars (over the lifetime of the instrument) suggests that this correlation may be less strong than previously thought [199]. For the study of EBL attenuation, blazar variability is both a nuisance and an opportunity.

Variability could be a nuisance because measuring the spectral steepening of a source is more difficult when such spectrum is changing constantly. In the case of the LAT, or any other space-based instrument, a precise measurement of the high energy spectrum of a source requires long integration times, and thus, the timeaverage steepening is what is actually measured. The impact of blazar variability has already been probed with the simulation and analysis described above (see sec. 
5.3.1.2), and as shown, it did not prevent a correct determination of the collective level of EBL attenuation experienced by blazars as a function of redshift. It should be noted however, that blazar variability is not well understood (this is something that GLAST will measure), and that the variability model used in the simulation might differ significantly from reality.

Blazar variability could also represent an advantage. The energy cut-off observed in a given blazar should be the same independently of the flaring state of the source, if due to EBL absorption. This would constitute a powerful check of the effectiveness of individual blazars as probes of the EBL.

\subsubsection{Spectral Blazar Evolution}

An observation of a redshift-dependent effect does not guarantee actual absorption by EBL background. There would be a possibility that spectral evolution of $\gamma$-ray blazars might coincidentally mimic EBL attenuation. For example, if blazars that formed in the early universe suffered more internal attenuation than younger blazars, a similar effect could be observed. Such possibility has been proposed by Reimer [226] after modeling the intrinsic absorption of $\gamma$-rays with photons from the accretion disk and broad-line region of blazars during periods of strong accretion. Given the blazar emission model considered in this study, and assuming a correlation between accretion history and black hole mass, it was found that the intrinsic opacity of blazars is redshift-dependent (through black hole mass evolution), and thus, it mimics EBL attenuation.

In scenarios like the one just considered, the intrinsic energy cut-offs (the same applies for flux-ratios) observed in blazars would vary blazar-to-blazar, and thus, the energy cut-offs for blazars in a given redshift bin would have larger scattering with respect to the mean than in EBL-only absorption scenarios (where the scattering is introduced by the measurement and not by the underlying physics). This would 
allow at least an upper limit on EBL attenuation by looking at the least-attenuated energy-cutoff (or flux ratio) in a particular redshift bin. Furthermore, intrinsic opacity is likely to change within each blazar during different emission states, allowing thus to constrain the nature of the observed energy cut-off.

Another possibility of spectral blazar evolution derives from the variability feature of blazars and indications that their spectra can become harder when flaring; if the flaring probability changes with time there is a chance of finding a larger than expected fraction of quiescent blazars at high redshift and therefore a dimmer emission. Theorists will have to decide the likelihood of an evolutionary conspiracy. Nevertheless, advancement could be made by complementary observations that reduce the likelihood of such conspiracies. For example, the electron - positron pair produced by EBL absorption belong to the highly-relativistic regime and could eventually emit a cascade of photons in the GeV range (sec. 5.5.3). Detection of this secondary radiation would confirm the attenuation and would put stronger constraints in the density and spectrum of EBL radiation. In general, as the blazar unified picture becomes more solid, it will become easier to distinguish real attenuation by EBL absorption from individual peculiarities at the source.

\subsubsection{Redshift Determination for GLAST blazars}

The analysis techniques described in this chapter will require redshift determinations for a large fraction of GLAST blazars. This is just another example of the importance of cross-wavelength studies: by using optical measurements of blazar redshifts, $\gamma$-ray observations can effectively probe the optical-UV EBL.

Given the importance of redshift determinations for this and other studies (blazar luminosity function, blazar evolution, bolometric flux measurements, etc.), the LAT collaboration has engaged the astronomical community in a common effort to carry out this type of multi-wavelength studies, which are necessary to maximize the science 
return from GLAST [95].

A brief description of the multiple on-going efforts to provide redshift determinations for GLAST blazars is given below. These include existing blazar catalogs with known counterparts (and redshifts) and other spectroscopy observations of blazars with unknown redshift.

\subsubsection{Existing Blazar Catalogs}

\section{3rd EGRET Catalog}

The third EGRET catalog contains 66 high-confidence blazar identifications with another 27 low-confidence identifications, and 170 unidentified sources ${ }^{11}$. Since GLAST is expected to detect sources very much fainter than those detected by EGRET, most of the blazars in the 3rd EGRET catalog will be observed by GLAST and their redshift information will be inherited.

The redshift distribution of the blazars in the 3rd EGRET catalog is shown in fig. 5.15. Redshifts have been measured for 62 (of the 66) high-confidence blazars (red line) and for 20 (of the 27) low-confidence blazars. The total distribution (black line) has mean $\langle z\rangle=0.97$ and a maximum of $z_{\max }=3.1$. This relatively small number of blazars already constitutes a substantial set of bright $\gamma$-ray sources to probe the EBL, and given a suitable intrinsic spectra (hard emission without energy cut-offs due to internal absorption) it could be used to validate -or refute- different EBL models from the literature (through the Fazio-Stecker Relation for example). 


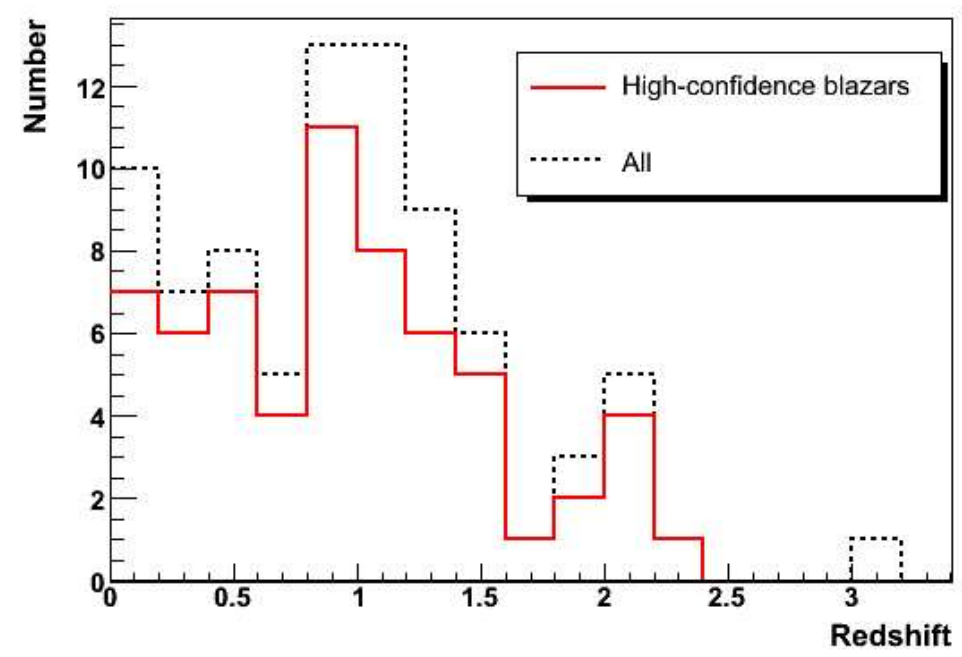

Figure 5.15: Redshift distribution for blazars in the 3rd EGRET catalog [106]. The catalog contains 66 high-confidence blazars (red line) and another 27 possible identifications. The total distribution (high-confidence+possible identifications) is shown in black.

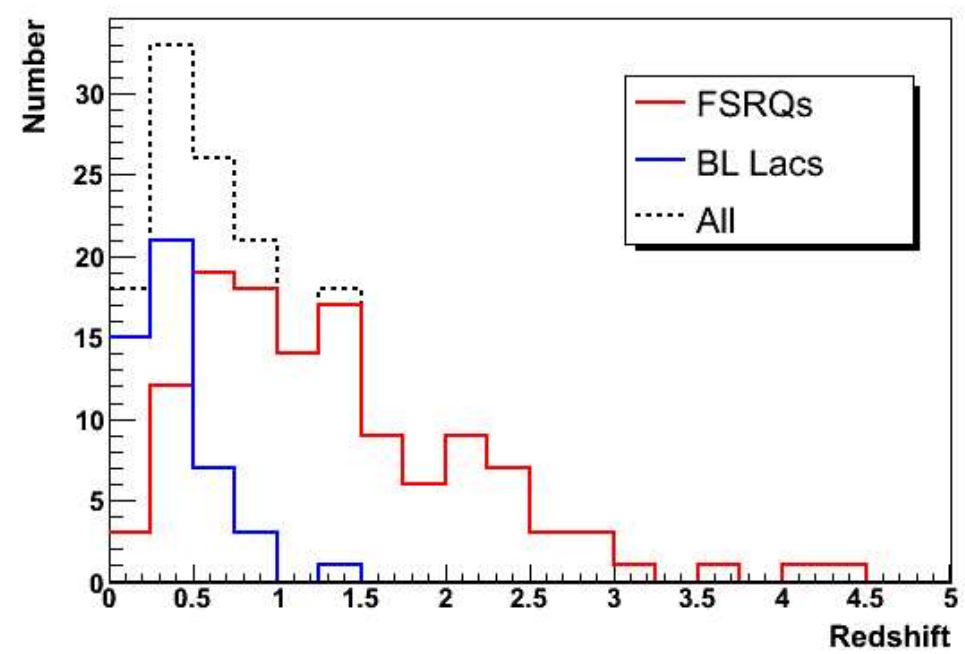

Figure 5.16: Redshift distribution of blazars (black) in the ASDC Catalog of blazars Vol. I [173]. Also shown: FSRQs (red) and BL Lacs (blue) distributions. 


\section{ASDC $^{12}$ Catalog of Known Blazars}

Massaro et al. [173] have compiled a list of blazars (detected at optical to X-ray wavelengths) which includes a large database of broad-band spectral energy distributions. Volume I of their catalog covers one quarter of the sky (Right Ascension: $0^{\circ}-90^{\circ}$ ) and consists of 239 high-confidence blazars (115 BL Lacs and 124 FSRQs), of which 171 have measured redshifts. The redshift distribution of this sample (see fig. 5.16) has a mean redshift $\langle z\rangle=1.06$ and a maximum of $z_{\max }=4.41$. A catalog that covers the whole sky has been announced for release at the end of 2007 .

\subsubsection{Catalogs of Blazar Candidates}

\section{Candidate Gamma-ray Blazar Survey (CGRaBS)}

In preparation for GLAST, Sowards-Emmered et al. [247] have compiled a sample of blazar candidates to increase the pool of well-studied AGN from which counterparts to the GLAST-detected sources will be obtained. A figure of merit that uses radio and X-ray observations as an input was trained on EGRET blazars, and then applied to objects in the Cosmic Lens All-Sky Survey (CLASS). The sources selected with this figure of merit have thus optical and X-ray fluxes similar to those of the EGRET blazars and are excellent blazar candidates to be detected by GLAST. This resulted in 710 blazar candidates in the northern sky, of which nearly half have archival classification and measured redshift (from the Quasar Catalog [272] and Sloan Digital Sky Survey [1]). Follow-up observations of the remainder sources have already resulted in 241 new identifications, most of them with measured redshifts. This sample is being extended to the southern sky by considering sources in the VLA (Very Large Array)

\footnotetext{
${ }^{11}$ Thanks to its better localization, GLAST will help identify most of the unidentified sources in the EGRET catalog.

${ }^{12}$ ASI (Agenzia-Spaziale-Italiana) Science Data Center
} 
survey, from which a similar number of $\gamma$-ray blazar candidates is expected. The redshift distribution of the identified FSRQs in the survey is shown in fig. 5.17.

\section{The Radio Optical X-ray ASDC (ROXA) Blazar Survey}

Turriziani et al. [269] have used radio observations from $\mathrm{NVSS}^{13}$, X-ray observations from RASS ${ }^{14}$, and optical magnitudes from GCSII $^{15}$ to select objects that, according to their ratio-to-optical and optical-to-X-rays flux ratios are good blazar candidates as explained in [212]. This resulted in over 7650 blazar candidates (see fig. 5.18), of which 500 are included in catalogs of known blazars. Identification through optical spectroscopy using SDSS and $2 \mathrm{dF}$ was pursued for a sub-sample of $\sim 800$ candidates. Approximately $63 \%$ of the candidates were confirmed as blazars, while another $13 \%$ of the sources kept their "candidate" label because although they have QSO optical spectrum no radio spectral information is available to determine if they have steep (nonblazar-like) or flat (blazar-like) radio spectrum. Extrapolation of these results to the whole sky yields over $\sim 5000$ expected blazars (70\% of 7650 ). How many of the blazar candidates in this survey will have $\gamma$-ray emission over the LAT sensitivity threshold remains an open question, but current estimates predict well over $\sim 1000$.

\subsubsection{Blazar Identification}

The existence of complete blazar catalogs at radio and X-ray wavelengths is a necessary but not sufficient condition towards the identification and redshift determination of $\gamma$-ray blazars. In general, gamma-ray instruments have poor angular resolution (as

\footnotetext{
${ }^{13}$ National Radio Observatory (NRAO) Very Large Array (VLA) Sky Survey is a 1.4 GHz continuum survey covering the sky north of -40 deg declination.

${ }^{14}$ ROSAT All-Sky Survey. ROSAT was an X-ray observatory that observed the sky between 1990 and 1999 .

${ }^{15}$ Guide Star Catalog II is an all-sky optical catalog based on 1" resolution scans of the photographic Sky Survey plates, at two epochs and three band-passes, from the Palomar and UK Schmidt telescopes.
} 


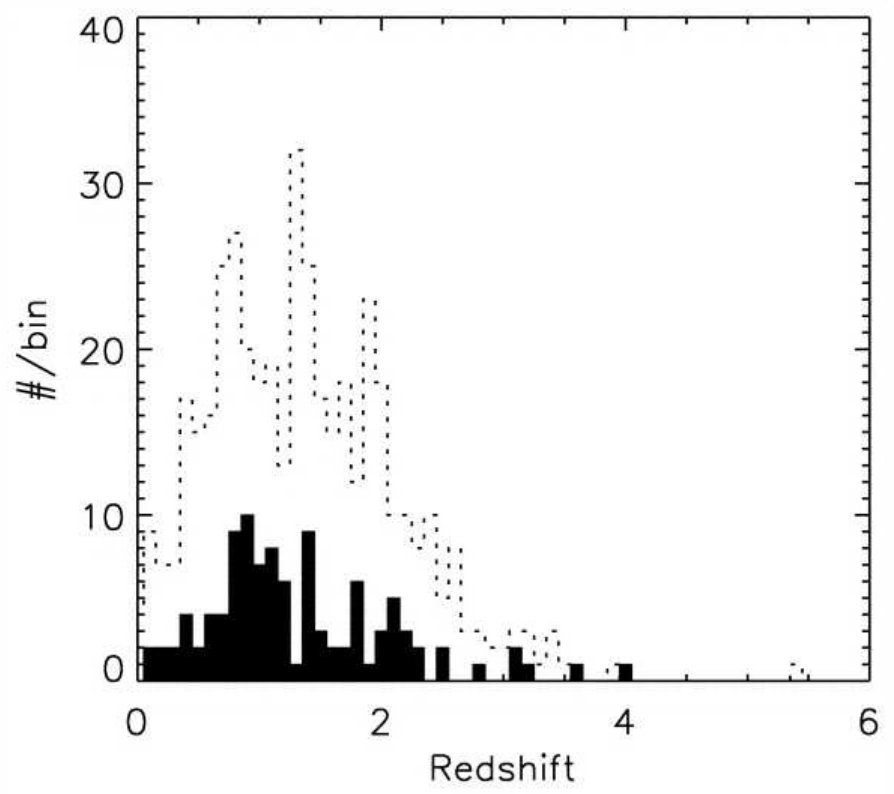

Figure 5.17: Redshift distribution of FSRQs in the Candidate Gamma-ray Blazar Survey [247] in the northern sky (dotted line). The redshift distribution of the FSRQs in the 3rd EGRET catalog is also shown for comparison (filled histogram).

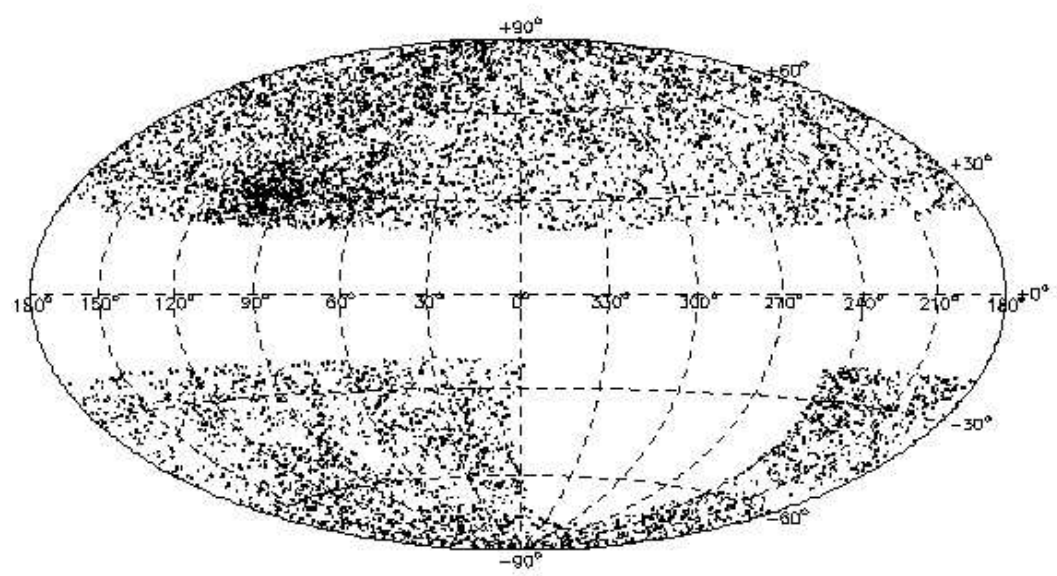

Figure 5.18: Blazar candidates in the ROXA survey, from [269]. 
compared to telescopes at other wavelengths), and this leads to large uncertainties in the position of their detected sources. This means that multiple counterparts (from different catalogs) can be found within the error box of a typical $\gamma$-ray source. This degeneracy is overcome with the use of figure-of-merit quantities that indicate the degree of correlation between the $\gamma$-ray source and its possible counterparts. Figure-of-merit quantities are obviously based on spatial coincidence, but they may also include additional information such as radio and X-ray fluxes, spectral indices, source luminosity, variability, etc. The likelihood of a possible counterpart is quantified by the figure-ofmerit and a threshold is set for positive identification. Identification techniques for blazars have already been established [180, 247], and in general they look for radio signatures (flux and spectral index) that are consistent with the correlation between FSRQs and $\gamma$-ray blazars discovered by EGRET.

\subsection{The Future}

\subsubsection{Unfolding of EBL density from Optical depth}

In contrast to previous analysis of $\mathcal{O}(\gtrsim 100 \mathrm{GeV})$ observations that are sensitive to the current-age EBL density $n(E, z=0)$, GLAST observations can be used to probe the EBL density evolution, which is particularly sensitive to the history of star formation and the effects of dust extinction in the early universe. Given the right conditions (enough suitable sources at the relevant redshifts, careful consideration of the causes of bias, etc.) this would be accomplished by measuring first the optical depth $\tau(E, z)$, and then unfolding the EBL photon density $n(E, z)$.

The analysis techniques presented in this dissertation are an effective way to detect the EBL attenuation of blazars, and in addition, the spectral analysis technique can be used to provide an effective (although rough) approximation of the observed optical 
depth $\tau(E, z)$. As an intermediate step towards a better understanding of the EBL, the FSR plot obtained from GLAST observations can be used to validate (or refute) EBL models from the literature like those introduced in Chapter 2 (Kneiske et al., Stecker et al., Primack et al.). Once the values of $\tau(E, z)$ measured by GLAST have been established, future EBL models can be constrained to reproduce such data.

A slightly different approach has been proposed by Vassiliev [271] to unfold the EBL. This technique assumes that both the measured optical depth and the expected EBL density can be expressed in parametric form, which allows to invert the expression

$$
\tau\left(E_{\gamma}, z\right) \propto \int_{0}^{z} \int_{\epsilon_{t h}}^{\infty} d z^{\prime} d \epsilon \frac{d l}{d z^{\prime}} \sigma\left(E\left(z^{\prime}\right), \epsilon\right) n_{E B L}\left(\epsilon, z^{\prime}\right)
$$

so that $n(\epsilon, z)$ can be expressed in terms of the measured values of $\tau(E, z)$. Although the calculations presented by Vassiliev in [271] are only valid for low-redshift sources $(z \ll 0.3)$, they can be generalized to any redshift.

\subsubsection{Joint spectral analysis with ground-based instruments}

The LAT, with a threshold well below $10 \mathrm{GeV}$, has access to the region of the gammaray spectrum that is not attenuated by the EBL (at any redshift). Observations in this range could be particularly useful for sources observed also at very high energy ( $\mathrm{E}$ $>100 \mathrm{GeV}$ ), since it would provide a reliable measurement of the intrinsic spectrum of the source.

As an example, two scenarios are considered for a $\gamma$-ray source at $\mathrm{z}=0.2$ (see fig. $5.19)$ :

(i) a soft power-law intrinsic spectrum $(\alpha=2.2)$ with nominal EBL absorption (Primack et al. 2005), and

(ii) a hard spectrum $(\alpha=2.0)$ with the EBL absorption increased by $20 \%$. 


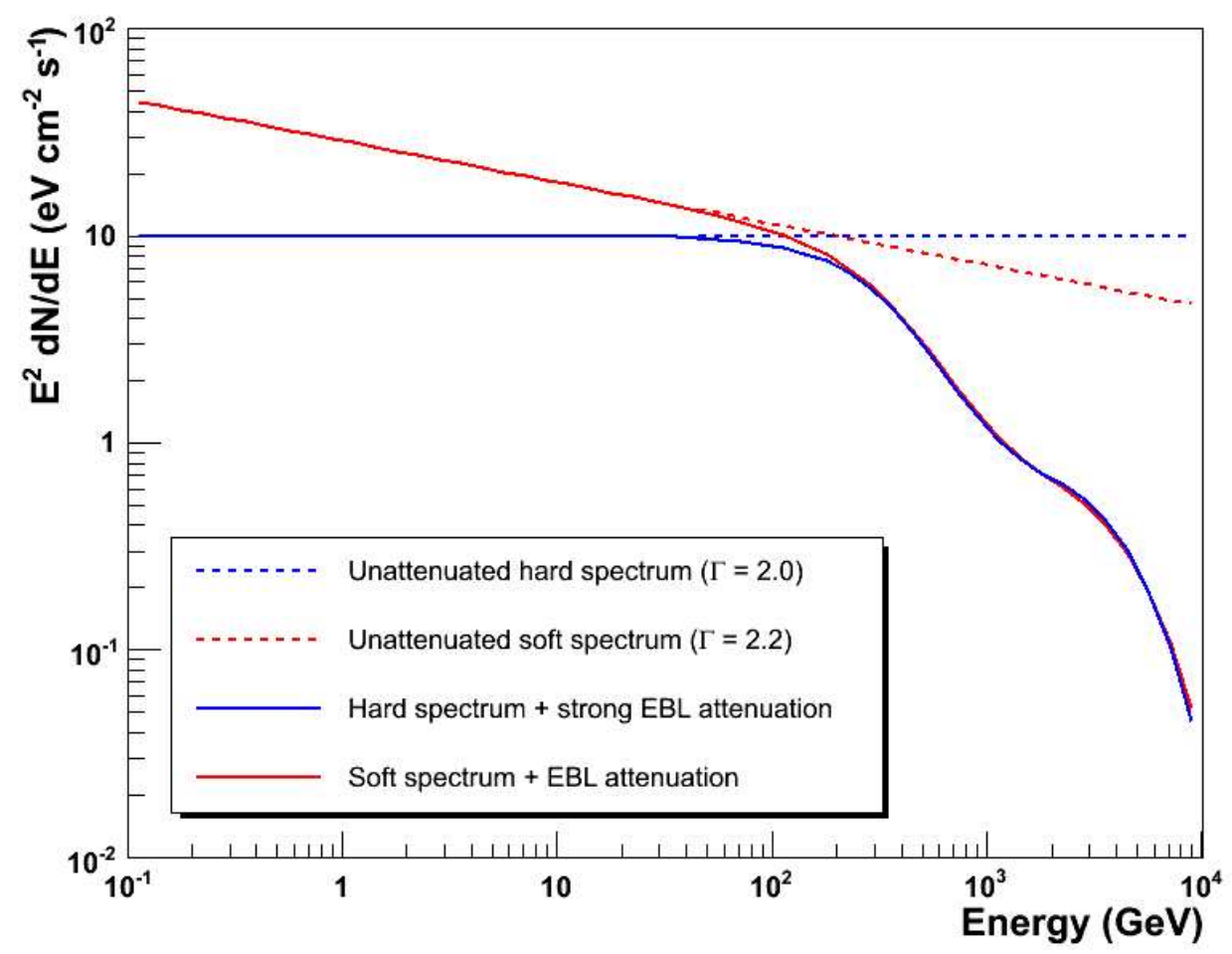

Figure 5.19: Two scenarios are considered for a $\gamma$-ray source at $z=0.2$ : a soft powerlaw intrinsic spectrum $(\alpha=2.2)$ with nominal EBL absorption according to Primack et al. 2005 (red solid line), and a hard spectrum $(\alpha=2.0)$ with the EBL absorption increased by $20 \%$ (blue solid line). These two scenarios are impossible to distinguish with observations above $100 \mathrm{GeV}$ only. The unattenuated spectra are also shown (dashed lines). 
Both scenarios are impossible to distinguish with data above $100 \mathrm{GeV}$ only, but joint observations by the LAT and ground-based telescopes could be used to eliminate the ambiguity and better constrain the optical-infrared EBL.

\subsubsection{Related EBL-Attenuation Phenomena}

\section{Gamma-rays cascading down in energy}

The interaction of $\gamma$-rays with EBL photons produces $e^{ \pm}$pairs that, by conservation of energy and momentum, are very energetic $\left(\sim E_{\gamma} / 2\right)$ and travel -at least initiallyin the direction of the original $\gamma$-ray. The pairs will subsequently Compton scatter on ambient photons (from the CMB and EBL), and as a result, secondary $\gamma$-rays with average energy $\sim 0.63\left(E_{\gamma} / 1 \mathrm{TeV}\right)^{2} \mathrm{GeV}$ will be produced ([52] and references therein). This chain reaction will continue until the nth-generation $\gamma$-rays have an energy such that $\tau\left(E_{n}\right) \ll 1$, which according to the EBL models is roughly $E_{n} \sim 10$ $\mathrm{GeV}$.

The observational manifestation of this "gamma-ray cascade" is determined by the distance traveled by the original $\gamma$-ray before the pair-production takes place (which in turn depends on the EBL density and the $\gamma$-ray energy), and the strength of the ambient magnetic field. Primary photons with $E_{\gamma} \sim 100 \mathrm{TeV}$ emitted by nearby blazars have a typical mean free path of a few megaparsecs $\left(1 \mathrm{Mpc} \simeq 3 \times 10^{22} \mathrm{~m}\right)$, which is small compared to the distance to the observer, but large enough for the photons to escape to intergalactic space (where the magnetic field is probably weaker, but remains unknown). The mean free path traveled by the relativistic electron (or positron) before Compton scattering a low energy photon from the background radiation is given by $\lambda_{e} \sim m_{e}^{2} c^{4} /\left(4 E_{\gamma} \sigma_{T} u_{B}\right)$, where $m_{e}$ is the electron mass, $\sigma_{T}$ is the Thomson cross section and $u_{B}$ is the energy density of the radiation background, which can be approximated by $u_{C M B}\left(u_{C M B} \gg u_{E B L}\right.$ as discussed in Chapter 2) 
and thus, $\lambda_{e} \sim\left(E_{\gamma} / 1 \mathrm{TeV}\right)^{-1} \mathrm{Mpc}$. This distance is also much less than the distance from the source to the observer, and in the presence of a magnetic field $B$, determines the amount of deflection experienced by the electron: $\theta=\lambda_{e} / R_{L} \simeq$ $1.3 \times 10^{-5}\left(E_{\gamma} / 1 \mathrm{TeV}\right)^{-2}\left(B / 10^{-20} \mathrm{G}\right)$, where $R_{L}=\gamma_{e} m_{e} c^{2} /(e B)$ is the Larmor radius of the electron.

\section{Formation of pair halos around blazars}

If the inter-galactic magnetic field (IGMF) is stronger than about $10^{-12} \mathrm{G}$, the relativistic electron(positron) will suffer a strong deflection, and its direction will become isotropic before the secondary $\gamma$-ray is produced through inverse Compton scattering. This would result in the formation of a extended halo around the source whose $\gamma$-ray radiation is isotropic. The extent and spectral profile of this halo (discussed in [7]) depend on the EBL density and the source spectrum. From the observational point of view, compact halos (radio $\ll 1 \mathrm{Mpc}$ ) would be probably brighter (total energy divided by surface) but also impossible to observe because of its small angular size from the observer point of view $\left(\ll 0.03^{\circ}\right.$ for a source at $\left.z=0.5\right)$ given the current PSF of spaceborne instruments. In that case, the flux emitted directly by the source and by the halo would overlap in a single point source. Very extended halos in the other hand, would be fainter and therefore difficult to detect. Nevertheless, the existence of halos in any form would imply $\gamma$-ray emission by misaligned blazars (i.e. any radio-loud AGN) that could be detected by GLAST as individual $\gamma$-ray sources (if bright enough to be resolved), or by their contribution to the extragalactic $\gamma$-ray background [143, 50].

If the IGMF is weak, no halo will be formed because the relativistic electron would not suffer any significant deflection before inverse Compton scattering takes place. In this case, the cascade photons will seem to come from the same point source and their time delay (with respect to the original emission) is dominated by the energy- 
dependent angular spread time $\Delta t \sim 1 / 2\left(\lambda_{e} / c\right) \theta^{2} \simeq 6 \times 10^{3}\left(E_{\gamma} / 1 \mathrm{TeV}\right)^{-5}\left(B / 10^{-20} \mathrm{G}\right)^{2} \mathrm{~s}$ for $\theta \ll 1$. Indeed, the existence of a hitherto undiscovered $\mathrm{GeV}$ emission during flaring and quiescence states has been proposed for nearby blazars ([52] for Mrk 501, and [78] for H1426+428), with a spectrum and duration that depend on the field strength and thus, could be used to probe the IGMF. The GeV flux levels predicted are consistent with existing EGRET upper limits and should be detectable with GLAST for $B_{I G M F} \lesssim 10^{-16} \mathrm{G}(\mathrm{Mrk} 501 ;[52])$, or $B_{I G M F} \lesssim 10^{-18} \mathrm{G}(\mathrm{H} 1426+428 ;[78])$.

\section{EBL Attenuation of the Extragalactic $\gamma$-ray Background}

The extragalactic $\gamma$-ray background (EGRB) detected by EGRET [251, 259] is believed to be a superposition of unresolved sources of high-energy emission. Since blazars are the dominant class of extragalactic sources at this energies, there is wide consensus that blazars (and radio-galaxies to a lesser extent) contribute significantly to this emission $(25 \%$ to $100 \%$ of the observed intensity according to different predictions). Contributions from other plausible sources have also been suggested (see [259] for relevant references): galaxy clusters, dark matter annihilation, particles accelerated in shock waves associated with large-scale cosmological structure formation, distant $\gamma$-ray bursts, etc.

Independently of their origin, high energy $(E \gtrsim 10 \mathrm{GeV}) \gamma$-rays emitted at cosmological distances will experience absorption by the EBL, and therefore, the EGRB spectrum is expected to steepen above $\sim 10 \mathrm{GeV}$. The presence of this spectral feature was not effectively probed by EGRET because its effective area decreased rapidly above $10 \mathrm{GeV}$ (due to backsplash self-veto, discussed in Chapter 4). GLAST, with a much better sensitivity at these energies, will measure the spectrum of the EGRB and test if it is actually dominated by blazar emission [233] or any other cosmological population.

As described above, extragalactic sources with spectra extending well into the $\mathrm{TeV}$ 
regime would lead to significant amounts of lower energy $\gamma$-rays through a photon cascade. Evidence of such cascade emission at the individual or collective level would thus provide information about the sources emissivity at very high energies, for which direct observation is impossible because of EBL absorption. Coppi \& Aharonian [50] applied this principle to the energy flux observed by EGRET above $100 \mathrm{MeV}$, and calculated an upper limit of $\sim 1-3 \times 10^{50} \mathrm{erg} \mathrm{s}^{-1}$ to the very high energy emissivity of the universe. This limit, that is not too high, applies to any cosmological population with significant emission above $\gtrsim 1 \mathrm{TeV}$. GLAST observations could strongly reduce this limit by resolving a significant fraction of the extragalactic background into individual blazars, implying therefore that typical blazar spectra only extends up to $\sim 100 \mathrm{GeV}$. In any case, EBL results from blazar observations must be consistent with the observed extragalactic $\gamma$-ray background.

\subsection{Summary and Conclusions}

GLAST is expected to detect thousands of blazars with redshifts up to z $\sim 5$ given its improved sensitivity with respect to previous missions, and reasonable extrapolations of the $\log (N)-\log (S)$ plot measured by EGRET. By measuring the attenuation of these sources, GLAST will probe the UV-optical EBL density and its evolution over cosmic time. Indeed, if enough sources are observationally available at the relevant redshifts, EBL attenuation of $\gamma$-ray sources could become a direct cosmological probe of the high-redshift universe. Statistical analyses which involve a large number of sources, as those presented in this dissertation, are a powerful tool to distinguish intrinsic peculiarities of blazar spectra from redshift-dependent EBL attenuation.

These are not the only methods. EBL absorption can also be measured by using blazar-emission models to predict the unattenuated spectrum of blazars through fitting of multi-wavelength data. Furthermore, blazars are not the only class of ex- 
tragalactic $\gamma$-ray sources, GRBs (gamma-ray bursts) are also located at cosmological distances (observed up to $z \gtrsim 6$ ) and will experience the same kind of EBL attenuation [134]. Little is known about the high energy emission $(E>1 \mathrm{GeV})$ of GRBs and thus it is difficult to predict the feasibility of EBL studies this type of objects. These two possibilities constitute independent types of analysis with respect to the one illustrated here, and when considered together, they will validate and complement each other.

It is hard to overstate the importance of multi-wavelength observations for $\gamma$-ray blazars. EBL studies with the analysis techniques introduced here will require the identification and redshift determination for a large number of GLAST blazars. This is not a trivial undertaking, but the effort will be well rewarded.

Even after observation of a redshift-dependent effect, the possibility would remain that the spectral evolution or observational selection of $\gamma$-ray blazars mimic EBL absorption. Future analyses will have to address the likelihood of such scenarios. GLAST observations, in any case, will provide an important constraint. 


\section{Chapter 6}

\section{Conclusion}

This dissertation explores the capability of GLAST to detect the effects of EBL absorption in the spectra of gamma-ray blazars. This is motivated by the exciting possibility of using this effect to probe the optical-UV EBL and its evolution over cosmic history.

Determination of the EBL flux remains one of the most challenging problems in astrophysics. So far, most direct measurements of the EBL have yielded results with strong systematic uncertainties due to the model-dependent subtraction of local and galactic foregrounds. On the other hand, lower limits on the EBL obtained by stacking the light of resolved galaxies are not satisfying either, since the possibility would remain that a significant population of dim sources remains undetected or that a truly diffuse component exists on the EBL flux.

The use of gamma-ray observations to study the EBL offers a powerful and elegant way to overcome some of the problems faced with direct measurements. This technique has already been applied to $\mathcal{O}(\gtrsim 100 \mathrm{GeV})$ observations of blazars by groundbased gamma-ray telescopes to put upper limits on the near-infrared cosmic background. Observation of just two blazars by the HESS collaboration has already provided a huge leap towards answering one of the outstanding questions in the study 
of the cosmic infrared background, namely, the existence of a large near-infrared background excess.

Study of the optical-UV EBL with GLAST is greatly anticipated because GLAST observations will probe the EBL as a function of cosmic time. The fact that the EBL models reviewed in Chapter 2 predict gamma-ray opacities that are so different should not be taken lightly. These models represent the best attempts to describe the EBL based on the current understanding of the formation and evolution of matter in the universe. So, the lack of a unified picture reflects that there are some issues that are still not understood. GLAST has the potential to distinguish among these models.

Measurement of the EBL from the study of its effects on the spectra of blazars is not a simple task. This is due mostly to the fact that blazars and their emission processes are not well understood. Conversely, blazars can not be completely understood if the effects of EBL absorption are not considered. GLAST represents a great opportunity to break this vicious circle by allowing the study of EBL attenuation with a large population of sources that are distributed over a wide range of redshifts. The analysis techniques introduced in this dissertation make use of this advantage by studying the collective behavior of blazars as a function of redshift. Techniques of this type offer a powerful way to separate the common level of attenuation due to the EBL from the intrinsic peculiarities that vary blazar to blazar.

Even after observation of a redshift-dependent attenuation in the spectra of blazars, the possibility would remain that the spectral evolution or observational selection of $\gamma$-ray blazars mimic EBL absorption. Future analyses will have to address the likelihood of such scenarios. GLAST observations, in any case, will provide an important constraint.

The type of analysis presented in this dissertation is not the only way to measure the effects of EBL absorption. Indeed, blazars are not the only class of extragalactic 
$\gamma$-ray sources that can be used to probe the EBL. Study of the EBL by spectral modeling of blazars, or with GRB observations, would constitute and independent type of analysis to the one presented here, with different systematics, and when considered together they will validate and complement each other.

The potential of GLAST to probe the EBL depends on great measure on its ability to mitigate the ACD backsplash self-veto problem that affected EGRET. This is accomplished by making use of a segmented ACD that reduces the effective area over which the backsplash probability is integrated. The compliance of the ACD design to this goal, and the effects of backsplash on the event reconstruction and $\gamma$ ray acceptance of the LAT have been studied by the LAT collaboration with detailed Monte Carlo simulations. The capability of these simulations to describe backsplash effects has been verified in this dissertation by measuring the backsplash probability with as-built flight detectors during a beam test of the LAT calibration unit at CERN in 2006.

In conclusion, the great window for very high energy $\gamma$-rays that GLAST will open will also provide unique insight into the optical-UV universe. This will lay a path for a deeper understanding of the universe for many years to come. 


\section{Bibliography}

[1] Abazajian, K., et al. 2005, AJ, 129, 1755

[2] Aharonian, F. A., et al. 2006, Nature, 440, 1018

[3] Aharonian, F. A., et al. 2006, ApJ, 636, 777

[4] Aharonian, F. A., et al. 2004, Nature, 432, 75

[5] Aharonian, F. A. 2000, NewA, 5, 377

[6] Aharonian, F. A., et al. 1999, A\&A, 349, 11

[7] Aharonian, F. A., Coppi, P., \& Völk, H. J. 1994, ApJ, 423, L5

[8] Altieri, B., et al. 1999, A\&A, 343, 59

[9] Amelino-Camelia, G., et al. 1998, Nature, 393, 319

[10] Arendt, R. G., et al. 1998, ApJ, 508, 74

[11] Atwood, W. B. 2007, LAT Event Analysis \& Performance, LAT Science Working Group (SWG) Review, February 2, 2007

[12] Atwood, W. B., et al. 2007, Design and Initial Tests of the Tracker-Converter of the Gamma-ray Large Area Space Telescope, in preparation 
[13] Atwood, W. 2005, GLAST Calibration and Analysis Group Meeting Minutes (08/15/05), available online at http://wwwglast.slac.stanford.edu/software/AnaGroup/

[14] Atwood, W., et al. 2003, LAT Beam Test Rationale, LAT-TD-00006-01

[15] Atwood, W., et al., 2000, NIM A 446, 444

[16] Barker, E., Pirzkal, N., et al. 2006, NICMOS Instrument Handbook, Version 9.0, (Baltimore: STScI).

[17] Barger, A. J., et al. 2001, AJ, 122, 2177

[18] Barger, A. J., et al. 2001, AJ, 121, 662

[19] Barnett et al. 1996, Phys. Rev. D54, 118

[20] Bernstein, R., Freedman, W. L., \& Madore, B. F. 2002, ApJ, 571, 56

[21] Bevington P. R., Robinson, D. K. 1992, Data Reduction and Error Analysis for the Physical Sciences, 2nd Ed. (New York: McGraw-Hill, Inc.)

[22] Biller, S. D., et al. 1998, Phys. Rev. Let., 80, 2992

[23] Biller, S. D., et al. 1995, ApJ, 445, 227

[24] Blain, A., et al. 1999, ApJL, 512, 87

[25] Blanch, O., \& Martinez, M. 2005, APh, 23, 598

[26] Blanch, O., \& Martinez, M. 2005, APh, 23, 588

[27] Blanch, O., Lopez, J., \& Martinez, M. 2003, APh, 19, 245

[28] Blanford, R. D., \& Znajek, R. L. 1977, MNRAS, 179, 433

[29] Bloom, S. D., Marscher, A. P. 1996, ApJ, 461, 657 
[30] Bogaert, G. 2001, CAL Pre-Electronics Module (PEM) Specification, (LAT Document: LAT-SS-00240)

[31] Bogges, N. W., et al. 1992, ApJ, 397, 420

[32] Bond, J. R., Carr, B. J., Hogan, C. 1986, ApJ, 306, 428

[33] Borden, T. 2002, LAT TKR Tray Assembly, Ladder Assembly, (LAT Document: LAT-DS-00026)

[34] Breiman, L., et al. 1984, Classification and Regression Trees, (New York: Chapman \& Hall)

[35] Brigida, M., et al. 2002, Signal simulation in silicon strip detectors (SSDs) and Digit package development, (LAT Document: LAT-TD-01058)

[36] Brown, T. M., et al. 2000, AJ, 120, 1153

[37] Bromm, V., \& Larson, R. B. 2004, ARA\&A, 42, 79

[38] Bruzual, G. A., \& Charlot, S. 1993, ApJ, 405, 538

[39] Burrows, A., \& Liebert, J. 1993, Rev. Mod. Phys., 65, 301

[40] Cambresy, L., et al. 2001, ApJ, 555, 563

[41] Checktman, A, et al., 2005, Shaped Readout Noise in $C A L$, available online at: http://wwwglast.slac.stanford.edu/IntegrationTest/SVAC/Instrument_Analysis/ Workshop-4/Talks/CAL_readout_noise_study.pdf

[42] Chen, A., Reyes, L. C. \& Ritz, S. M. 2004, ApJ, 608, 686

[43] Chiang, J., \& Mukherjee, R. 1998, ApJ, 496, 752 
[44] Chupp, E. L., 1996, High Energy Solar Physics, ed.: Ramaty, R., et al., AIP Conf. Proc., 374, 3

[45] Churazov, E., et al. 2006, preprint (astro-ph/0608250)

[46] Cillis, A. N., \& Hartman, R. C. 2005, ApJ, 621, 291

[47] Clements, D. L., et al. 1999, A\&A, 346, 383

[48] Collmar, W. 2001, The Universe in Gamma Rays: Gamma-Ray Emission of Active Galaxies, Chapter 12, edited by Schönfelder, V., (Berlin: Springer-Verlag)

[49] Coppi, P. \& Aharonian, F. A. 1999, ApJ, 521, L33

[50] Coppi, P. \& Aharonian, F. A. 1997, ApJ, 487, L9

[51] Cortina, J. et al. 2005, Proc. 29th ICRC, Pune, 5-359

[52] Dai, Z. G., et al. 2002, ApJ, 580, L7

[53] de Jager, O. C., \& Stecker, F. W. 2002, ApJ, 566, 738

[54] de Jager, O. C., Stecker, F. W., \& Salamon, M. H. 1994, Nature, 369, 294

[55] de Naurois, M., Holder, J. et al. 2002, ApJ 566, 343

[56] Dermer C. D. , 2006, ApJ, submitted (astro-ph/0605402)

[57] Dermer, C. D., Schlickeiser, R., \& Mastichiadis, A. 1992, A\&A, 256, 27

[58] Devriendt, J. E. G., Guiderdoni, B. 2000, A\&A, 363, 851

[59] Diehl, R. 2001, The Universe in Gamma Rays: Gamma-Ray Production and Absorption Processes, Chapter 2, edited by Schönfelder, V., (Berlin: SpringerVerlag) 
[60] do Couto e Silva, E., \& Ritz, S. 2004, LAT Science Verification Analysis and Calibration Plan, LAT-MD-04446

[61] do Couto e Silva, E., et al. 2001, NIM A 474, 19

[62] Dole, H., et al. 2006, A\&A, 451, 417

[63] Dole, H., et al. 2004, ApJS, 154, 87

[64] Donea, A-C, \& Protheroe, R. J. 2003, APh, 18, 377

[65] Dube, R. R., Wickes, W. C., \& Wilkinson, D. T. 1979, ApJ, 232, 333

[66] Dunlop, J. S., \& Peacock, J. A. 1990, MNRAS, 247, 19

[67] Dwek, E., et al. 2005, ApJ, 635, 784

[68] Dwek, E., \& Krennrich, F. 2005, ApJ, 618, 657

[69] Dwek, E. 2001, The Extragalactic Infrared Background and its Cosmological Implications, Proceedings of IAU Symposium, 204, 389

[70] Dwek, E., et al. 1998, ApJ, 508, 106

[71] Dwek, E., \& Arendt, R. G. 1998, ApJL, 508, 9

[72] Dwek, E., \& Slavin, J. 1994, ApJ, 436, 696

[73] Edelstein, J., Bowyer, S., \& Lampton, M. 2000, ApJ, 539, 187

[74] Eidelman, S., et al. 2004, Particle Data Group, Phys. Lett. B, 592

[75] Elbaz, D., et al. 2002, A\&A, 384, 848

[76] Elbaz, D., et al. 1999, A\&A, 351, L37

[77] Fall S. M., Charlot, S., \& Pei, Y.C. 1996, ApJ, 464, L43 
[78] Fan, Y. Z.; Dai, Z. G.; Wei, D. M. 2004, Astron. Astrophys., 415, 483

[79] Fazio, G. G., et al. 2004, ApJS, 154, 39

[80] Fazio, G. G., \& Stecker, F. W. 1970, Nature, 226, 135

[81] Feynman, R. 1998, Quantum Electrodynamics (Advanced Book Classics), (Boulder: Westview Press)

[82] Fichtel, C. E., \& Trombka, J. I. 1997, Gamma-Ray Astrophysics: New Insight Into the Universe (Washington, DC: NASA Reference publication 1386)

[83] Finkbeiner, D. P., Davis, M., \& Schlegel, D. J. 2000, ApJ, 544, 81

[84] Fishman, G. J., et al. 1994, ApJS, 92, 229

[85] Fixsen, D. J., et al. 1998, ApJ, 508, 123

[86] Fossati G. et al., 1998, MNRAS, 299, 433

[87] Frayer, D. T., et al. 2006, ApJ, 647, L9

[88] Fruwirth, R. 2000, Data Analysis Techniques for High-Energy Physics, 2nd ed. (Cambridge: Cambridge University Press)

[89] Funk, B., et al. 1998, APh, 9, 97

[90] Gardner, J. P., Brown, T. M., \& Ferguson, H. C. 2000, ApJL, 542, 79

[91] GEANT4 Web Page: http://wwwasd.web.cern.ch/wwasd/geant4/geant4.html

[92] Ghisellini, G., \& Madau, P. 1996, MNRAS, 280, 67

[93] Giommi, P., et al. 2006, Simulated and Real Blazar Surveys, LAT Blazars and Other AGN Science Group Face-toFace Meeting, SLAC (March 4th, 2006), available online at: http://www.cenbg.in2p3.fr/ftp/astropart/glast/agn_group/meeting_page.htm 
[94] Giommi, P., et al. 2005, A\&A, 445, 843

[95] GLAST LAT Collaboration, Multi-wavelength Coordination Webpage, available online at http://confluence.slac.stanford.edu/Multiwavelength + Coordinating + Group

[96] GLAST LAT Performance, available online at http://wwwglast.slac.stanford.edu/software/IS/glast_lat_performance.htm

[97] Gorjian, V., Wright, E. L., \& Chary, R. R. 2000, ApJ, 536, 550

[98] Gould, R. S., \& Schreder, G. 1966, Phys. Ref. Let., 16, 252

[99] Granato, G. L., Danese, L., Franceschini, A., 1997, ApJ, 486, 147

[100] Greisen, K. 1968, Lecture at the Brandeis Summer Institute in Physics

[101] Guy, J., et al. 2000, A\&A, 359, 419

[102] Haarsma, D. B., \& Partridge, R. B., 1998, ApJL, 503, 5

[103] Hacking, P., \& Soifer, B. 1991, ApJL, 367, 49

[104] Halzen, F., \& Martin, A. D. 1984, Quarks \& Leptons: An Introductory Course in Modern Particle Physics, (New York: John Wiley \& Sons, Inc)

[105] Hanna, D. S., et al. 2002, Nucl. Inst. Method. A, 491, 126

[106] Hartman, R. C. , et al. 1999, ApJS, 123, 79

[107] Hauser, M., \& Dwek, E. 2001, ARA\&A, 39, 249

[108] Hauser, M. G., et al. 1998, ApJ, 508, 25

[109] Heger, A. et al. 2003, ApJ, 591, 288 
[110] Helou, G. 1991, The Interpretation of Modern Synthesis Observation s of Spiral Galaxies, (San Francisco: Astron. Soc. Pac.)

[111] Hinton, J. A. 2004, NewAR, 48, 331

[112] Holder, J. 2006, preprint (astro-ph/0611598)

[113] Hoyle, F. 1953, ApJ, 118, 513

[114] Hopkins, A. M., Irwin, M. J., \& Connolly, A. J. 2001, ApJL, 558, 31

[115] Huffer, M. 2003, GLT Electronics Module, (LAT Document: LAT-TD-01545)

[116] Huffer, M. 2003, The ACD Electronics Module (AEM), (LAT Document: LATTD-00639)

[117] Huffer, M. 2003, The Command/Response Unit (CRU), (LAT Document: LATTD-01547)

[118] Huffer, M. 2003, The Event Builder Module (EBM), (LAT Document: LATTD-01546)

[119] Huffer, M. 2003, The Tower Electronics Module (TEM), (LAT Document: LATTD-00605)

[120] Hughes, R., et al. 2007, The Trigger and On-board Filter of the GLAST LAT, Poster Presentation, 1st GLAST Symposium, Palo Alto, Ca.

[121] Hunter, S. D., et al. 1997, ApJ, 481, 205

[122] Jackson, J. D. 1998, Classical Electrodynamics, 3rd edition, (New York: John Wiley \& Sons, Inc)

[123] Jacobson, T., Liberati, S., \& Mattingly, D. 2006, Annals of Physics, 321, 150

[124] Jacobson, T., et al. 2004, PhRvL, 93, 1101 
[125] Jelley, J. V. 1966, Phys. Ref. Let., 16, 479

[126] Jiang, L., et al. 2006, preprint (astro-ph/0611453)

[127] Jones B., \& Tompkins, W. 1998, A Phycisist's Guide to Kalman Filters

[128] Johnson, W. N., et al. 2000, The Construction and Performance of the CsI Hodoscopic Calorimeter for the GLAST Beam Test Engineering Module, AASHEAD Meeting, 5, 43.22

[129] Johnson, W. N., et al. 1993, ApJS, 86, 693

[130] Jorstad, S., et al. 2001, ApJS, 134, 181

[131] Juvela, M., Matilla, K., \& Lemke, D. 2000, A\&A, 360, 813

[132] Kalman, R. E. 1960, Transactions of the ASME-Journal of Basic Engineering, $82 \mathrm{D}, 35$

[133] Kashlinsky, A. 2006, preprint (astro-ph/0610943)

[134] Kashlinsky, A. 2005, ApJL, 633, 5

[135] Kashlinsky, A. 2005, PhR, 409, 361

[136] Kashlinsky, A., et al. 2005, Nature, 438, 45

[137] Kashlinsky, A., \& Odenwald, S. 2000, ApJ, 528, 74

[138] Kashlinsky, A., et al. 1996, ApJ, 470, 681

[139] Kataoka, J., et al. 2003, ApJ, 650, 659

[140] Kawara, K., et al. 1998, A\&A, 336, 9

[141] Kelsall, T., et al. 1998, ApJ, 508, 44 
[142] Kennicutt, R. C. 1998, ARA\&A, 36, 189

[143] Kneiske, T. M., \& Mannheim, K. 2005, , 4-1

[144] Kneiske, T., et al. 2004, A\&A, 413, 807

[145] Knoll, G. F. 1989, Radiation Detection and Measurement, (New York: John Wiley \& Sons Inc.)

[146] Kowalski, M., Ringwald, A., \& Tu H. 2002, Phys. Lett. B, 529

[147] Kraeueter, R., \& Bourgeois, P. 2003, Crystal Detector Element Specification (LAT Document: LAT-SS-00239)

[148] Krennrich, F., \& Dwek, E. 2003, Proc. 28th ICRC, Japan, 2667

[149] Kuhr, H., Witzel, A., Pauliny-Toth, I.I.K., \& Nauber, U. 1981, A\&AS, 45, 367

[150] Krennrich, F., et al. 2001, ApJ, 560, L45

[151] Lagache, G., \& Puget, J.-L. 2000, A\&A, 355, 17

[152] Lagache, G., et al. 1999, A\&A, 344, 322

[153] Lamb, R. C. \& Weekes, T. C. 1987, HE-UHE Behaviour of Accreting X-ray Sources, Conference Proceedings, 333

[154] Lapi, A. et al. 2006, ApJ, 650, 42

[155] Leahy, J. P., \& Perley, R. A. 1991, ApJ, 102, 537

[156] Leinert, C., et al. 1998. Astron. Astrophys. Suppl., 127, 1

[157] Lichti, G., \& Georgii, R. 2001, The Universe in Gamma Rays: Instruments, Chapter 3, edited by Schönfelder, V., (Berlin: Springer-Verlag)

[158] Liru, Y.-C., et al. 2002, NASA Technical Report TP-2002-210780 
[159] Liu, H. T., \& Bai, J. M. 2006, ApJ, 653, 1089

[160] Lyutikov, M. 2003, NewAR, 47, 513

[161] Macminn, D., \& Primack, J.R. 1996, Space Sci. Rev., 75, 413

[162] Madau, P., \& Pozzetti, L. 2000, MNRAS, 312, 9

[163] Madau, P., Pozzetti, L., \& Dickinson, M. 1998, ApJ, 498, 106

[164] Madau, P., \& Phinney, E. S. 1996, ApJ, 456, 124

[165] Madau, P., et al. 1996, MNRAS, 283, 1388

[166] Madejski, G., Reimer, A., et al. 2007, Science Goals Document of the LAT Blazar and other AGNs science group, available online at http://confluence.slac.stanford.edu/display/SCIGRPS/Blazars + and + Other + AGNs

[167] Malkan, M. A., \& Stecker, F. W. 2001, ApJ, 555, 641

[168] Malkan, M. A., \& Stecker, F. W. 1998, ApJ, 496, 13

[169] Maraschi, L., Fossati, G. 1996, Gamma-ray Emitting AGN Conference, (astro$\mathrm{ph} / 9612084)$

[170] Maraschi, L, Ghisellini, G., \& Celotti, A. 1992, ApJL, 397, 5

[171] Marconi, A., et al. 2004, MNRAS, 351, 169

[172] Martin, C., Hurwitz, M., \& Bowyer S. 1991. ApJ, 379, 549

[173] Massaro, E. et al. 2005, Multifrequency Catalog of Blazars, Vol I (0h-6h) (Rome: Aracne Editrice) available online at http://www.asdc.asi.it/bzcat/

[174] Mastichiadis, A., \& Kirck, J. A. 1997, A \& A, 320, 19

[175] Matilla, K. 2003, ApJ, 591, 119 
[176] Mattila, K. 1990, The Galactic and Extragalactic Background Radiation, IAU Symp., 139

[177] Matsuhara, H., et al. 2000, A\&A, 361, 407

[178] Matsumoto, T., et al. 2005, ApJ, 626, 31

[179] Matsumoto, T., et al. 2000, ISO Surveys of a Dusty Universe, Lect. Notes, (Berlin: Springer-Verlag)

[180] Mattox et al. 1997, ApJ, 481, 95

[181] Mattox, J. R., et al. 1997, ApJ, 476, 692

[182] Mattox, J. R., et al. 1996, ApJ, 461, 396

[183] Mazin, D., \& Raue, M. 2007, preprint (astro-ph/0701694)

[184] McDonald, P., Scherrer, R. J., \& Walker, T. P. 2001, PhRvD, 63, 3001

[185] Meegan, C., et al. 2007, The GLAST Burst Monitor, in preparation

[186] Metcalfe, L., et al. 2003, A\&A, 407, 791

[187] Michelson P. 2001, in AIP Conf. Proc. 587, Gamma-Ray Astrophysics Symposium 2001, ed. S. Ritz, N. Gehrels, \& C. R. Schrader (New York: AIP), 713

[188] Michelson P., et al. 2007, The Large Area Telescope on the GLAST Mision, in preparation

[189] Mighell, K. J. 1999, ApJ, 518, 380

[190] Moiseev, A., et al. 2007, The Anti-Coincidence Detector for the GLAST Large Area Telescope, in press 
[191] Moiseev, A. A., et al. 2004, Astroparticle Physics, 22, 275

[192] Morselli, et al. 2000, Nucl. Phys. B 85

[193] Moskalenko, I., et al. 2006, preprint (astroph/0609768)

[194] Mücke A., et al. 2003, APh, 18, 593

[195] Mücke A., \& Pohl, M. 2000, MNRAS, 312, 177

[196] Mukherjee, R. et al. 1997, ApJ, 490, 116

[197] Mukherjee, R., et al. 1995, ApJ, 445, 189

[198] Murthy, J., et al. 1999, ApJ, 522, 904

[199] Nandikotkur, G., et al 2007, ApJ, 657, 706

[200] Narumoto T., and Totani, T., 2006, ApJ, 643, 81

[201] NASA CGRO Science Support Center, EGRET Technical Information, available online at http://cossc.gsfc.nasa.gov/docs/cgro/egret/egret_tech.html

[202] NASA Explores, Models of the Electromagnetic Spectrum student sheet, available online at http://nasaexplores.nasa.gov

[203] Nelson, D. \& Johnson, R. 2003, Tracker Front End Readout ASIC Specification, (LAT Document: LAT-TD-00169)

[204] Nikishov, A. I. 1961, Sov. Phys. JETP, 41, 549

[205] Nishijima, K. et al. 2005, Proc. 29th ICRC, Pune, 5, 327

[206] Noda, M., et al. 1994, ApJ, 428, 363

[207] Nolan, P., et al. 1996, A\&AS, 120, 61 
[208] Olive, K. A., Steigman, G., \& Walker, T. P. 2000, PhR, 333, 389

[209] Olsen, J. 2003, Specification of the GLAST Tracker Readout Controller Electronics ASIC, (LAT Document: LAT-TD-00170)

[210] Oh, S. P. 2001, ApJ, 553, 25

[211] Ormes, J. F., et al. 2006, GLAST LAT Background Review, (LAT Document: LAT-TD-08316)

[212] Padovani, P. 2006, preprint (astro-ph/0610545)

[213] Padovani, P., Giommi, P. 1995, ApJ, 444, 567

[214] Papovich, C., et al. 2004, ApJS, 154, 70

[215] Pei, Y. C., \& Fall, S. M. 1995, ApJ, 454, 69

[216] Pei, Y. C., Fall, S. M., \& Hauser, M. G. 1999, ApJ, 522, 6042

[217] Perkins, D. H. 1987, Introduction to High Energy Physics (Menlo Park, CA: AddisonWesley)

[218] Peterson, B. M. 1997, An Introduction to Active Galactic Nuclei, (Cambridge: University Press)

[219] Picozza, P., et al. 2006, preprint (astro-ph/0608697)

[220] Piro, L. 1997, 191st AAS Meeting, \#55.01; Bulletin of the American Astronomical Society, 29, 1303

[221] Prialnik, D. 2000, An Introduction to the Theory of Stellar Structure and Evolution (Cambridge, UK: Cambridge University Press)

[222] Primack, J. R., Bullock, J. S., \& Somerville, R. S. 2005, preprint (astroph/0502177). Optical depth tables obtained via private communication. 
[223] Primack, J. R., Bullock, J. S., \& Sommerville, R. S. 1999, APh, 11, 93

[224] Puget, J-L, et al. 1999, A\&A, 345, 29

[225] Puget, J-L, et al. 1996, A\&A, 308, 5

[226] Reimer, A. 2007, The redshift-dependence of gamma-ray absorption in the environments of strong-line $A G N$, in preparation

[227] Reimer, A. 2007, private communication

[228] Renault, C., et al. 2001, A\&A, 371, 771

[229] Reynolds, C. S., et al. 1997, MNRAS, 291, 403

[230] Rieger, F. M., Bosch-Ramon, V., \& Duffy, P. 2006, based on a talk at "The multimessenger approach to unidentified gamma-ray sources", Barcelona/Spain, July 2006, preprint(astro-ph/0610141)

[231] Rochester, L. 2007, LAT Analysis Overview, LAT Science Working Group (SWG) Review, February 2, 2007

[232] Sadrozinski, H., \& Ohsugi, T. 2001, GLAST LAT Silicon Detector Specification, (LAT Document: LAT-TD-00011)

[233] Salamon, M. H., \& Stecker, F. W. 1998, ApJ, 493, 547

[234] Salpeter, E. E. 1955, ApJ, 121, 161

[235] Salvaterra, R., \& Ferrara, A. 2003, MNRAS, 339, 973

[236] Santos, M. R., Bromm, V., \& Kamionkowski, M. 2002, Mon. Not. R. Astron. Soc. 336, 1082

[237] Sambruna, R., Maraschi, L., \& Urry, C. M. 1996, ApJ, 463, 444 
[238] Sazonov, S., et al. 2007, A\&A, 462, 57

[239] Schelgel, D. J., Finkbeiner, D. P., \& Davis, M. 1998, ApJ, 500, 525

[240] Schönfelder, V. 2001, The universe in Gamma-rays, Chapter 1, (Berlin: Springer-Verlag)

[241] Schönfelder, V., et al. 1993, ApJS, 86, 657

[242] Schroedter, M. 2005, ApJ, 624, 638

[243] Serjeant, S., et al. 2004, ApJS, 154, 118

[244] Sikora, M., Begelman, M. C., \& Rees, M. J. 1994, ApJ, 421, 153

[245] Smith, A. J. 2005, Proc. 29th ICRC, Pune, 10-227

[246] Soifer, B. T., et al. 1987, ApJ, 320, 238

[247] Sowards-Emmered, D. et al. 2005, ApJ, 626, 95

[248] Spada, M. et al. 2001, MNRAS, 325, 1559

[249] Spergel, D. N., et al. 2006 , preprint (astro-ph/0603449)

[250] Spergel, D. N., et al., 2003, ApJS, 148, 175

[251] Sreekumar, P., et al. 1998, ApJ, 494, 523

[252] Stanev, T., \& Franceschini, A. 1998, ApJL, 494, 523

[253] Stecker, F. W., Malkan, M. A., \& Scully, S. T. 2006, ApJ, 648, 774

[254] Stecker, F. W., \& Glashow, S. L. 2001, APh, 16, 97

[255] Stecker, F. W., \& Salamon, M. H. 1996, ApJ, 464, 600

[256] Stecker, F. W., \& de Jager, O. C. 1993, AAS, 183, 2906 
[257] Stecker, F. W., de Jager, O. C., \& Salamon, M. H. 1992, ApJL, 390, 49

[258] Stecker, F. W. 1969, ApJ, 157, 507

[259] Strong, A. W., Moskalenko, I. V., \& Reimer O. 2004, ApJ, 613, 956

[260] Strong, A. W., Moskalenko, I. V., \& Reimer O. 2004, ApJ, 613, 962

[261] Swensen, E., et al. 2001, GLAST Tracker Mechanical Design: PDR Design Review Document, (LAT Document: LAT-TD-00489)

[262] Thompson, D. J., et al. 1993, ApJS, 86, 629

[263] Thompson, R. I., et al. 2007, 657, 669

[264] Timmer J., \& Koenig, M. 1995, A \& A, 300, 707

[265] Tomimatsu, A., \& Takahashi, M. 2003, ApJ, 592, 321

[266] Toller, G. N. 1983, ApJL, 266, 79

[267] Tosti, G., et al. 2006, Blazar Light Curves Simulation, GLAST Blazars and Other AGNs Science Group Face-toFace Meeting, SLAC (March 4th, 2006), available online at: http://www.cenbg.in2p3.fr/ftp/astropart/glast/agn_group/meeting_page.htm

[268] Tumlinson, J., Shull, J. M., Venkatesan, A. 2003, ApJ, 584, 608

[269] Turriziani, et al 2006, in prep.

[270] Urry C.M. and Padovani P., 1995, PASP, 107, 803

[271] Vassiliev, V. V. 2000, APh, 12, 217

[272] Véron-Cetty, M. P., \& Véron, P. 2003, A\&A, 412, 399

[273] Von Klien, M., et al. 2004, SPIE, 5488, 763 
[274] Wai, L. 2007, SUSY 2006, Conf. Proc., preprint(astro-ph/0701884)

[275] Weinberg, S. The first three minutes: A Modern View of the Origin of the Universe, 2nd Ed., (New York: Basic Books)

[276] Wilks, S. S. 1938, Ann. Math. Stat., 9, 60

[277] Witten, E. 1996, Nucl. Phys. B, 471, 135

[278] Wren, D. N. 2004, Detecting the High-Energy Emission from Gamma-ray Bursts with EGRET and GLAST, PhD Thesis, University of Maryland at College Park (LAT Document: LAT-TH-07960)

[279] Wright, E. L. 2001, ApJ, 553, 538

[280] Wright, E. L., \& Reese, E. D. 2000, ApJ, 545, 43

[281] Zdziarski, A. \& Svensson, R. 1989, A\&A, 344, 551

[282] Zhang, B., \& Meszaros, P. 2004, International Journal of Modern Physics A, 19,2385 